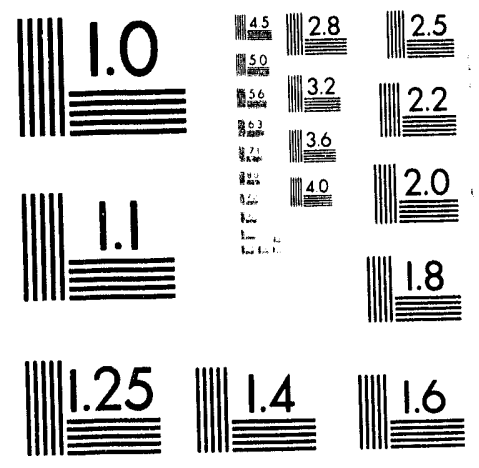



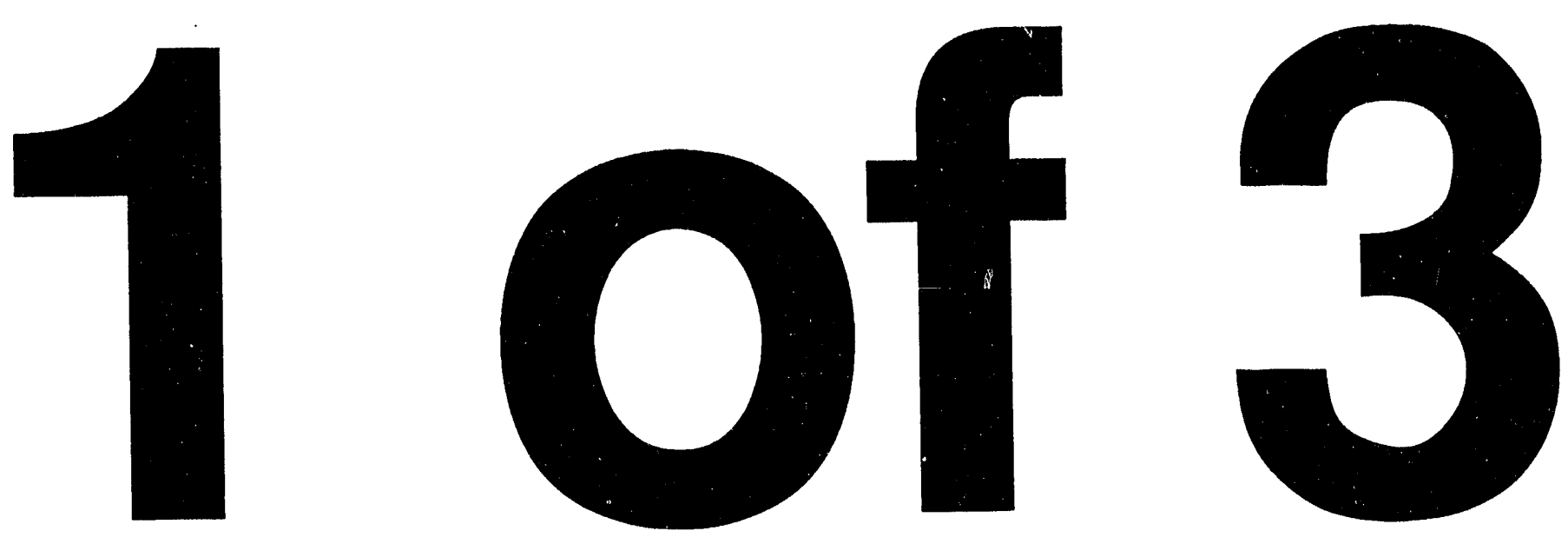
LBL-34923

\section{Modern Integral Equation Techniques for Quantum Reactive Scattering Theory}

Scott Michael Auerbach

Ph.D. Thesis

Department of Chemistry

University of California

and

Chemical Sciences Division Lawrence Berkeley Laboratory

University of California

Berkeley, California 94720

November 1993 


\title{
Modern Integral Equation Techniques for Quantum Reactive Scattering Theory
}

\author{
Copyright $@ 1991$
}

by Scott Michael Auerbach

The U.S. Department of Energy has to right to use this thesis for any purpose whatsoever including the right to reproduce all or any part thereof 


\title{
Modern Integral Equation Techniques for Quantum Reactive Scattering Theory
}

\author{
Scott Michael Auerbach
}

\begin{abstract}
Rigorous calculations of cross sections and rate constants for elementary gas phase chemical reactions are performed for comparison with experiment, to ensure that our picture of the chemical reaction is complete. We focus on the $\mathrm{H} / \mathrm{D}+\mathrm{H}_{2} \rightarrow$ $\mathrm{H}_{2} / \mathrm{DH}+\mathrm{H}$ reaction, and use the time independent integral equation technique in quantum reactive scattering theory.

We examine the sensitivity of $\mathrm{H}+\mathrm{H}_{2}$ state resolved integral cross sections $\sigma_{v^{\prime} j^{\prime}, v j}(E)$ for the transitions $(v=0, j=0)$ to $\left(v^{\prime}=1, j^{\prime}=1,3\right)$, to the difference between the Liu-Siegbahn-Truhlar-Horowitz (LSTH) and double many body expansion (DMBE) ab initio potential energy surfaces (PES). This sensitivity analysis is performed to determine the origin of a large discrepancy between experimental cross sections with sharply peaked energy dependence and theoretical ones with smooth energy dependence. We find that the LSTH and DMBE PESs give virtually identical cross sections, which lends credence to the theoretical energy dependence.

To facilitate quantum calculations on more complex reactive systems, we develop a new method to compute the energy Green's function with absorbing boundary conditions $(\mathrm{ABC})$, for use in calculating the cumulative reaction probability. The method is an iterative technique to compute the inverse of a non-Hermitian matrix which is based on Fourier transforming time dependent dynamics, and which requires very little core memory. The Hamiltonian is evaluated in a sinc-function based discrete variable representation (DVR), which we argue may often be superior to the fast Fourier transform method for reactive scattering. We apply the resulting power series Green's function to the benchmark collinear $\mathrm{H}+\mathrm{H}_{2}$ system over the energy range
\end{abstract}


0.37 to $1.27 \mathrm{eV}$. The convergence of the power series is stable at all energies, and is accelerated by the use of a stronger absorbing potential.

The practicality of computing the ABC-DVR Green's function in a polynomial of the Hamiltonian is discussed. We find no feasible expansion which has a fixed and small memory requirement, and is guaranteed to converge. We have found, however, that exploiting the time dependent picture of the ABC-DVR Green's function leads to a stable and efficient algorithm. The new method, which uses Newton interpolation polynomials to compute the time dependent wavefunction, gives a vastly improved version of the power series Green's function. We show that this approach is capable of obtaining converged reaction probabilities with very straightforward accuracy control.

We use the ABC-DVR-Newton method to compute cross sections and rate constants for the initial state selected $\mathrm{D}+\mathrm{H}_{2}(v=1, j) \rightarrow \mathrm{DH}+\mathrm{H}$ reaction. We obtain converged cross sections using no more than 4 Mbytes of core memory, and in as little CPU time as 10 minutes on a small workstation. With these cross sections, we calculate exact thermal rate constants for comparison with experiment. For the first time, quantitative agreement with experiment is obtained for the rotationally averaged rate constant $k_{v=1}(T=310 K)=1.9 \times 10^{-13} \mathrm{~cm}^{3} \mathrm{sec}^{-1} \mathrm{molecule}^{-1}$. The $J$-shifting approximation using accurate $J=0$ reaction probabilities is tested against the exact results. It reliably predicts $k_{v=1}(T)$ for temperatures up to $700 K$, but individual $(v=1, j)$-selected rate constants are in error by as much as $41 \%$. 


\section{Dedication}

To my beautiful wife Sarah, the light of my life 


\section{Acknowledgments}

I am grateful for the patience and encouragement from my research advisor, Prof. William H. Miller, with whom scientific interaction has always been a most exhilarating experience. Most of all, I would like to thank Bill for keeping me in graduate school. I would also like to acknowledge the friendship and guidance from Prof. Claude Leforestier. In addition, I thank Prof. Andrew Vogt (Georgetown University) for stimulating my interest in Green's functions, Prof. David Chandler for providing a wonderful teaching environment, and Prof. Robert G. Littlejohn for sharing his insight on semiclassical mechanics.

I have had the pleasure of knowing some very special postdocs in the Miller group. In particular, I would like to thank Dr. John Z. H. Zhang for gently introducing me to the real world of quantum reactive scattering calculations. I am obliged to Dr. Daniel T. Colbert for helping to sustain my interest in this subject. I am grateful to Dr. Gerrit C. Groenenboom for giving me the confidence to hurdle any numerical obstacle. Finally, I thank Dr. Peter Saalfrank for opening my eyes to the greater world of science.

I can not express how lucky I feel to have worked with Mrs. Cheryn Gliebe. Her invaluable assistance and friendship throughout my graduate career has made my years at Berkeley truly delightful.

I am grateful to the members of the Miller group past and present: Dr. Tamar Seideman, Dr. Gerhard Stock, Dr. Uwe Manthe, Dr. Agathe Untch, Dr. Nancy Makri, Dr. Beverly Grayce, Dr. Yan-Tyng Chang, Dr. Lionel F. X. Gaucher, Dr. Rigoberto Hernandez, Srihari Keshavamurthy, J. Daniel Gezelter, Ward H. Thompson, and Bruce W. Spath for all the help and friendship they have given me. I would like to give special thanks to Dr. Rigoberto Hernandez for skillful assistance with data and text processing, and to J. Daniel Gezelter for superb system administration.

It is not possible for me to list by name all the wonderful friends who have sustained me through the years; I am indebted to you all. I woud like to give special thanks to Maria C. Longuemare, David A. Hecht, Dr. Avery N. Goldstein, and 
Michael A. Olshavsky for looking after me - I really needed it. I am also grateful to Dr. John N. Gehlen for running a great softball club, and Dr. Joel S. Bader for teaching me my outside topic.

I have the best parents in the whole world. Their nurturing love has given me the confidence to make a difference. And their wonderful example has taught me that people always come first.

I do not think I can ever know how much I missed Sarah during the first three years in Berkeley. Thankfully, we are together again. I could never have finished this dissertation without her love and encouragement. We did it, honey!

This research was supported by a National Science Foundation graduate fellowship, and by the Director, Office of Energy Research, Office of Basic Energy Sciences, Chemical Sciences Division of the U.S. Department of Energy under Contract No. DE-AC03-76SF00098. 


\section{Contents}

Dedication $\quad$ i

Acknowledgments $\quad$ iii

Table of Contents $\quad$ v

List of Tables $\quad$ ix

List of Figures $\quad$ xi

1 General Introduction 1

1.1 First Philosophical Principles . . . . . . . . . . . . . . . . . 1

1.2 Molecular Beams and Collision Theory . . . . . . . . . . . . . 3

1.3 Theoretical Practices Old and New . . . . . . . . . . . . 8

1.4 Looking Ahead .................... . . 13

$\begin{array}{ll}\text { References } & 14\end{array}$

2 Sensitivity Analysis for $\mathbf{H}+\mathbf{H}_{2} \quad 17$

2.1 Introduction . . . . . . . . . . . . . . . . 17

$2.2 S$-matrix Kohn Formulation . . . . . . . . . . . 20

2.3 Functional Sensitivity Analysis ................ 24

2.4 The Potentials: LSTH vs. DMBE . . . . . . . . . . . . . 26

2.5 The Dynamics: Results and Discussion . . . . . . . . . . . . 27

2.6 Concluding Remarks .................. 35

$\begin{array}{ll}\text { References } & 37\end{array}$

3 Power Series Green's Function $\quad 41$

3.1 Introduction . . . . . . . . . . . . . . . . . 41

3.2 General Methodology . . . . . . . . . . . . . . . . . . . . . . . . . . . . . . . . 45

3.3 Power Series Green's Function . . . . . . . . . . . . . . . . . 47 


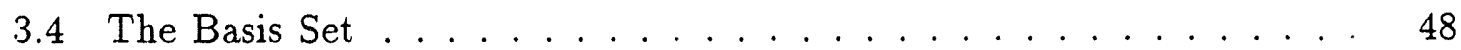

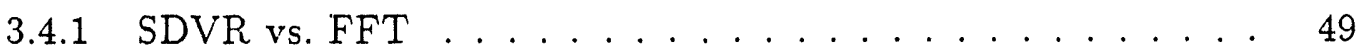

3.4.2 SDVR of the Free Particle Propagator . . . . . . . . . 49

3.5 Multidimensional Generalization . . . . . . . . . . . . . . . 52

3.6 Summary of the Methodology . . . . . . . . . . . . . . . 53

3.7 Results and Discussion . . . . . . . . . . . . . . . . . 54

3.7.1 The Coordinates . . . . . . . . . . . . . . 54

3.7 .2 The Absorbing Potential . . . . . . . . . . . . 55

3.7.3 Reaction Probabilities . . . . . . . . . . . . . . 56

3.7 .4 Convergence Tests . . . . . . . . . . . . . . . . 59

3.8 Concluding Remarks . . . . . . . . . . . . . . . . 64

3.8 .1 Reactive Scattering . . . . . . . . . . . . . . . . 65

3.8 .2 Path Integration $\ldots \ldots \ldots \ldots 6$

$\begin{array}{ll}\text { References } & 70\end{array}$

4 The Newton Algorithm $\quad \mathbf{7 7}$

4.1 Introduction . . . . . . . . . . . . . 77

4.2 Quantum Reactive Scattering Formulation . . . . . . . . . . 79

4.2 .1 Formal Theory . . . . . . . . . . . . . . . . 79

4.2.2 Absorbing Boundary Conditions . . . . . . . . . . . 84

4.3 Polynomial Expansions . . . . . . . . . . . . . . . . . . . 86

4.3.1 Direct Expansion . . . . . . . . . . . . . . . . 99 90

4.3.2 Indirect Expansion . . . . . . . . . . . . . . . . . . 112

4.4 Quantum Reactive Scattering Calculations . . . . . . . . . . . 117

4.4 .1 Reaction Probabilites . . . . . . . . . . . . . . . . 117

4.4 .2 Convergence Tests . . . . . . . . . . . . . . . . 118

4.5 Concluding Remarks . . . . . . . . . . . . . . . . . 123

$\begin{array}{ll}\text { References } & 126\end{array}$

5 The $\mathrm{D}+\mathrm{H}_{2}(v=1)$ Rate Constant 131

5.1 Introduction . . . . . . . . . . . . . . . 131

5.2 General Methodology . . . . . . . . . . . . . . . . . . . . . 134

5.2.1 General Rate Constant Formulae . . . . . . . . . . . . . 135

5.2.2 ABC Formulation of Quantum Reactive Scattering . . . . . 136

5.3 Defining the Linear System _ . . . . . . . . . . . . . . . 137

5.3 .1 The Coordinates . . . . . . . . . . . . . . . 137

5.3 .2 The Basis Set . . . . . . . . . . . . . . . . . . . 142

5.3.3 The Reference Scattering State . . . . . . . . . . . . 145

5.3.4 The Absorbing Potential . . . . . . . . . . . . . . . . . 151

5.3.5 Summary of the Methodology . . . . . . . . . . . . . 152 
5.4 Results and Discussion ..................... 152

5.4.1 Reaction Probabilities and Cross Sections . . . . . . . . 152

5.4 .2 Rate Constants . . . . . . . . . . . . . . . . . . 162

5.5 Concluding remarks . . . . . . . . . . . . . . . 168

$\begin{array}{ll}\text { References } & 171\end{array}$

6 General Conclusions $\quad 175$

6.1 The $\mathrm{H} / \mathrm{D}+\mathrm{H}_{2}$ System . . . . . . . . . . . . . . . . 175

6.2 Integral Equations . . . . . . . . . . . . . . . . 176

6.3 And Beyond ... . . . . . . . . . . . . . . . . . . . . . . 179

6.3.1 Quantum Reaction Rate Theory . . . . . . . . . . . . . 179

6.3.2 Gas Phase Reaction Dynamics . . . . . . . . . . . . . . 180

6.3.3 Physical Chemistry . . . . . . . . . . . . . . . 181

$\begin{array}{lc}\text { References } & 182\end{array}$ 


\section{List of Tables}

2.1 Fixed angle saddle point comparisons of LSTH/DMBE . . . . . . . . 27

5.1 Optimized convergence parameters for scattering calculations . . . . . 153

5.2 Exact and approximate theoretical rate constants for $\mathrm{D}+\mathrm{H}_{2} \ldots \ldots$. . 167

5.3 Experiment and theory for the $\mathrm{D}+\mathrm{H}_{2}$ rate constant . . . . . . . 168 


\section{List of Figures}

1.1 Collinear $\mathrm{H}+\mathrm{H}_{2}$ potential energy surface contours . . . . . . . . . 10

2.1 Experiment and theory for the $\mathrm{H}+\mathrm{H}_{2}$ cross section . . . . . . . . 19

$2.2 \mathrm{LSTH} / \mathrm{DMBE}$ potentials for collinear geometries . . . . . . . . . . 28

$2.3 \mathrm{LSTH} / \mathrm{DMBE}$ potentials for L-shaped geometries . . . . . . . . . . . 29

2.4 Partial cross sections for LSTH/DMBE with $J=0, E \ldots \ldots \ldots$

2.5 Product distributions for LSTH/DMBE with $J=0, E=1.2 \mathrm{eV} \ldots . \quad 32$

2.6 Partial cross sections for LSTH/DMBE with $J=10, E \ldots \ldots 33$

2.7 Product distributions for LSTH/DMBE with $J=10, E=1.2 \mathrm{eV}$. . 34

2.8 Experiment and theory for the $\mathrm{H}+\mathrm{H}_{2}$ cross section . . . . . . . 36

3.1 Configuration space for cumulative reaction probability . . . . . . 57

3.2 Cumulative reaction probabilitiy for $\mathrm{H}+\mathrm{H}_{2} \ldots \ldots \ldots$. . . . . 58

3.3 Convergence of $N(E)$ with respect to time step . . . . . . . . . 61

3.4 Convergence of $N(E)$ with respect to total propagation time . . . . 62

3.5 Effect on total propagation time of strong absorbing potential . . . . 63

4.1 Configuration space for reactant state selection . . . . . . . . . 88

4.2 Distorted Born expansion eigenvalue convergence test for $\mathrm{H}+\mathrm{H}_{2}$. . . 92

4.3 Distorted Born expansion eigenvalue convergence test for $\mathrm{H}+\mathrm{O}_{2}$. . . $\quad 93$

4.4 Newton interpolation sampling points . . . . . . . . . . . . 105

4.5 Convergence of Newton expansion for $g(z)=(E-z)^{-1} \ldots \ldots$. . . . 107

4.6 Scaled and shifted ABC Hamiltonian spectrum . . . . . . . . . . 108

4.7 Convergence of Newton expansion for $g\left(z_{\text {pole }}\right)=\left(E-z_{\text {pole }}\right)^{-1} \ldots$. . . 110

4.8 Convergence of Newton expansion for $u(z)=e^{-i z t} \ldots \ldots \ldots 115$

$4.9 \mathrm{D}+\mathrm{H}_{2}(v, j)$ reaction probabilities for $J=0, E=0.9 \mathrm{eV} \ldots \ldots \ldots$.

4.10 Convergence of probabilities with degree of Newton expansion . . . . 121

4.11 Convergence of probabilities with order of time quadrature . . . . . . 122

4.12 Convergence of probabilities with total propagation time . . . . . . . 124

5.1 Translational and rotational coordinate frames for A+BC system . . 139

5.2 Convergence with $z_{\max }$ of small $\mathrm{D}+\mathrm{H}_{2}$ reaction probabilities . . . . 155 
5.3 Convergence with $z_{\text {max }}$ of large $\mathrm{D}+\mathrm{H}_{2}$ reaction probabilities . . . 156

5.4 Reaction probabilities for the $\mathrm{D}+\mathrm{H}_{2}(v=1, j)$ system $\ldots \ldots \ldots . . .158$

5.5 Convergence with $K_{\max }$ of $\mathrm{D}+\mathrm{H}_{2}$ cross sections . . . . . . . . . 159

5.6 Dependence of partial cross sections on total angular momentum . . . 161

5.7 Reaction cross sections for the $\mathrm{D}+\mathrm{H}_{2}(v=1, j)$ system . . . . . . . 163

5.8 Exact and approximate rate constants for $\mathrm{D}+\mathrm{H}_{2}(v=1, j) \ldots \ldots 6$

5.9 Exact and approximate rate constants for $\mathrm{D}+\mathrm{H}_{2}(v=1,<j>) \ldots 169$ 


\section{Chapter 1}

\section{General Introduction}

We are interested in the theoretical study of gas phase chemical reactions from first physical principles. We accomplish this efficiently and with quantitative accuracy for the reactions studied. This is a remarkable achievement, both in practice and in principle.

\subsection{First Philosophical Principles}

We assume that physics can explain all chemical phenomena. This viewpoint, tacitly assumed by most modern scientists, exemplifies a philosophy known as reductionism [1]. The success of reductionism reported in this dissertation has significant implications for the authority of philosophies opposed to reductionism. To put these implications in perspective, we discuss here briefly the holisitic philosophical stance put forth by Aristotle (384-322 B.C.).

The distinction between Aristotelian philosophy and that of modern science is somewhat subtle, since Aristotle himself helped to define many fields in modern science, e.g. mechanics and biology. To illustrate this point, we describe some aspects of his physics [2]. When Aristotle arrived at Plato's academy, he found that Plato did not welcome scientific inquiry. Rather, Plato emphasized the reality of abstract ideals, which one could comprehend only by avoiding sensory perception. Aristotle developed a more practical ideology by postulating that reality consists in tangible 
objects which have both essential characteristics (comparable to Plato's ideals) and accidental characteristics. For example, it is essential that Scott has a brain in order to philosphize; but, for the same purpose it is only accidental that he has green eyes. In modern scientific language, Aristotle's tangible object is our mechanical system; his essential characteristics correspond to our Hamiltonian and other constants of the motion; his accidental characteristics translate to particular values of our dynamical variables. Thus, Aristotle gave philosophical grounding to the notion of dynamics, which has inspired our current mechanical ansatz.

Aristotle, whose father was a physician, was also fascinated by the nature of living organisms. In his seminal contribution to biology [3] Aristotle emphasized the importance of the structure-function relationship. He postulated that a biological structure can be understood fully in terms of the function it provides. For example, we easily understand the structure of a bird's wing given the bird's inclination to fly. However, if birds were to swim rather than to fly, the wing structure would become mysterious since it is ineffective for this purpose. Furthermore, it does not provide explanatory value, for Aristotle, to reduce conceptually the wing to bone, muscle, feathers, etc., because we find these biological structures in organisms which cannot fly. In short, Aristotle felt that function is philosophically more fundamental than structure; he thus put forth a holistic biology in which complex structures are understood in terms of the function they provide, rather than in terms of their constituent parts.

This mode of explanation is ubiquitous in modern biological studies. However, although this explanatory methodology proves useful to many biologists, their philosophy differs fundamentally from Aristotle's. In particular, most biologists believe that structure is philosophically more fundamental than function. For example, a modern biologist might analyze the chemistry of wing muscle from a hawk wing and a chicken wing to explain why the former can fly whereas the latter cannot. In general, most biologists believe in principle that chemistry can explain biological phenomena, although this may be difficult in practice, and hence not yet completely achievable. Taking this reductio ad infinitum, the modern scientist believes that the most complex phenomena can be explained by the most fundamental principles. Thus, the 
reductionism of modern science stands in stark contrast to the holism of Aristotle.

Both philosophical viewpoints have strengths and weaknesses. For example, Aristotle's holism provides a stunningly simple explanation for very complex structure. Unfortunately, in its simplicity it borders on tautology. On the other hand, developing a reductionist explanation often presents great difficulty. In addition, it poses the theological dilemma of reducing humanity to collections of fundamental particles. However, reductionism provides a less tautological explanation of observed phenomena. Since reductionism presents difficulty in practice, any instance of its successful implementation is remarkable. In this vein, we note in addition to the present work, a recent use of chemical physics to explain biological function in photosynthetic electron transfer [4]. It thus appears that the reductionist viewpoint is presently in vogue because it provides a mechanical, non-tautological explanation of natural phenomena.

At this point the author must take a stand. My love for physical chemistry's particular version of the reductionist endeavor leads me to believe that chemistry can be reduced to physics. However, in spite of mounting evidence for biological reductionism, it is difficult for the author to conceive of himself as nothing more than a bag of particles. As such, the author must advocate biological holism despite the burdensome paradox this presents. With these sentiments expressed, we proceed with physical chemistry.

\subsection{Molecular Beams and Collision Theory}

Physical chemists have long sought fundamental understanding of how molecules transform during chemical reactions. The development of molecular beam techniques $[5,6]$ beginning in the 1950's has facilitated the observation of bimolecular chemical reactions at the most microscopic, single collision level of detail. The ideal molecular beam scattering experiment involves a collision between reactants prepared in a well defined relative momentum and internal state, giving scattered products whose internal state is detected at a particular scattering angle. Although such a measurement has never been performed, experimentalists have made great progress 
toward this ideal experiment.

The observable quantity in molecular beam experiments is called the differential reaction cross section $d \sigma_{\mathbf{n}_{p}, \mathbf{n}_{r}}(v, \Omega) / d \Omega$ defined by [7]

$$
\begin{aligned}
d \Omega \times\left[d \sigma_{\mathbf{n}_{p}, \mathbf{n}_{r}}(v, \Omega) / d \Omega\right] \equiv & \frac{N\left(\mathbf{n}_{p}, \Omega\right)}{D\left(\mathbf{n}_{r}, v\right)} \\
\text { where } N\left(\mathbf{n}_{p}, \Omega\right)= & \text { number of products in state } \mathbf{n}_{p} \text { detected per } \\
& \text { unit time in solid angle range }(\Omega, \Omega+d \Omega) \\
& \text { (i.e. spherical flux), } \\
\text { and } D\left(\mathbf{n}_{r}, v\right)= & \text { number of reactants in state } \mathbf{n}_{r} \text { with velocity } \\
& v \text { crossing unit area per unit time } \\
& \text { (i.e. planar flux). }
\end{aligned}
$$

As such, the units of $d \sigma_{\mathbf{n}_{p}, \mathbf{n}_{r}}(v, \Omega) / d \Omega$ are area per solid angle. Another important observable is the integral reaction cross section $\sigma_{\mathbf{n}_{p}, \mathbf{n}_{r}}(v)$, which in subsequent chapters is simply referred to as the reaction cross section. This quantity is defined by $[7]$

$$
\sigma_{\mathbf{n}_{p}, \mathbf{n}_{r}}(v) \equiv \int d \Omega \times\left[d \sigma_{\mathbf{n}_{p}, \mathbf{n}_{r}}(v, \Omega) / d \Omega\right]
$$

where

$$
\int d \Omega=\int_{-1}^{+1} d \cos \Theta \int_{0}^{2 \pi} d \Phi=4 \pi
$$

Experimentalists measure integral reaction cross sections most directly in a bulb apparatus which does not detect the scattering angle dependence of product formation. A physical interpretation of the integral reaction cross section is the circular area centered at the target (i.e. one reactant collision partner) which, when crossed by the projectile (i.e. the other reactant collision partner), leads to a chemical reaction.

To illustrate these definitions, we consider a collision between two hard spheres (HS) with radii $r_{1}$ and $r_{2}$, yielding a total hard sphere radius $r=r_{1}+r_{2}$. The HS differential reaction cross section is $r^{2} / 4$, independent of scattering angle. The HS integral reaction cross section is $\pi r^{2}$, consistent with circular area of radius 
$r$. Both the HS differential and integral reaction cross sections are independent of the relative velocity $v$ because the HS interaction has no finite energy scale. The HS model grossly oversimplifies the collision event because actual molecular collisions involve continuous, anisotropic forces. Nevertheless, the HS model proves useful for estimating effective molecular sizes.

Molecular beam and bulb experiments have two goals. First, reaction cross sections can be transformed into reaction rate constants, which provide important kinetic information regarding chemical reaction rates. The rate constant with reactant state selection may be particularly important for technological applications. Indeed, a chemical mixture which reacts exothermically from one reactant state, and is inert from all other reactant states might provide a useful energy source to complement fossil fuels. For more fundamental reasons, we focus on the initial state selected rate constant for the $\mathrm{D}+\mathrm{H}_{2} \rightarrow \mathrm{DH}+\mathrm{H}$ reaction in Chapter 5 .

Molecular beam and bulb experiments also provide detailed information regarding the basic forces which control chemical reactions. Knowledge of these forces affords more profound understanding of the collision event, and may facilitate studying similar reactions in solution or on solid surfaces. Various authors refer to the process of surmising force laws from cross section data as inverse scattering or as the inversion problem. In the simple case of elastic scattering (i.e. collision partners with no internal structure), we can deduce the underlying force law in a straightforward manner from cross section data because the force is central. Large velocity cross sections provide information about steep repulsive walls at small relative separations, whereas low energy scattering gives information regarding attractive wells and long range potential tails.

Inverse scattering for more complicated collisions poses the challenge of constructing multidimensional potential functions, an arduous task for which no unique method of choice exists. Nevertheless, physical chemists can infer much of the underlying dynamics by studying how cross sections vary with reactant selection and product detection. (In this dissertation we focus on integral reaction cross sections, and hence do not consider the study of angular distributions.) For example, examining the product state distribution, i.e. $\sigma_{\mathbf{n}_{p}, \mathbf{n}_{r}}(v)$ vs. $\mathbf{n}_{p}$, indicates the extent of energy flow 
from reactant vibrations and rotations to those of the products. This analysis may lead to general principles in reaction dynamics. For example, the collinear Polanyi rules [7] predict that an early (late) barrier reaction is more efficiently promoted with translationally (vibrationally) excited reactants, yielding product state distributions which are vibrationally (translationally) hot. In addition, the dependence of integral reaction cross sections with initial translational energy indicates the presence of reaction barriers, and gives qualitative information about reaction timescales. With regard to the latter, sharp energy dependence signifies the formation of a collision complex, with the width of the sharp feature inversely proportional to the lifetime of the complex. Reaction dynamicists strive to observe such features (known as resonances), because they elucidate the energy level structure of the reactive transition state. In Chapter 2 we use both energy dependence and product state distribution analysis to examine $\mathrm{H}+$ para $-\mathrm{H}_{2} \rightarrow$ ortho $-\mathrm{H}_{2}+\mathrm{H}$ cross sections at relative velocities for which a recent bulb experiment has reported formation of an $\mathrm{H}_{3}$ collision complex $[8]$.

Theoretical reaction dynamics can contribute on many levels to our understanding of chemical phenomena. Occasionally, for example, theory can calculate an observable quantity which cannot be measured accurately. Since most dynamics experiments measure relative populations, theory can calibrate them by calculating the absolute cross section for a particular transition. On a more important level, theory contributes to our understanding of chemical phenomena by direct comparison with experiment. Comparisons with approximate calculations are indispensable in determining which aspects of the underlying physics control the reactivity. Alternatively, comparisons with accurate calculations help to ensure that our picture of the chemical reaction is complete. In this dissertation, we develop and apply accurate theoretical methods for describing electronically adiabatic atom-diatom reactive scattering.

Quantum mechanical reactive scattering theory [9-11] (QRS) provides the physical principles we use to represent reactive molecular collisions. Implementing QRS for electronically adiabatic collisions involves two steps: solving the electronic Schrödinger equation for various molecular configurations yielding a BornOppenheimer potential energy surface (PES); solving the resulting nuclear Schrödinger 
equation yielding the stationary scattering wavefunction. We do not focus on the electronic problem because we study molecular systems $\left(\mathrm{H}+\mathrm{H}_{2}\right.$ and $\left.\mathrm{D}+\mathrm{H}_{2}\right)$ for which a very accurate PES is known. Nevertheless, this is a crucial step in the accurate description of a molecular collision [12].

An asymptotic analysis of the scattering wavefunctio.. demonstrates the relationship between QRS and the differential reactive cross section. To make this relationship concrete, we define for an $N$ atom reactive system with chemical arrangements labeled by $\tau$, a set of $3 N$ dimensional Jacobi scattering coordinates $\left(\chi, \mathbf{R}_{\tau}, \mathbf{q}_{\tau}\right)$ for each chemical arrangement. We remove the center of mass vector $\chi$ leaving $3 N-3$ coordinates. The three coordinates $\mathbf{R}_{\tau}$ are the scattering coordinates, i.e. the vector which joins the centers of mass of the two collision partners. The radial scattering coordinate is $R_{\tau}=\left|\mathbf{R}_{\tau}\right|$. The $3 N-6$ coordinates $\mathbf{q}_{\tau}$ define the internal motions, i.e. the vibrations and rotations of both collision partners. The stationary scattering wavefunction $\Psi_{\mathbf{N} \tau}^{+}$is labeled by a collection of quantum numbers $N=(\mathbf{k}, \mathbf{n})$ which defines the initial momentum and internal state, respectively, in reactant arrangement $\tau$. The total system energy $E=E_{t}+\varepsilon_{\mathbf{n}}$, where $E_{t}=\hbar^{2} k^{2} / 2 \mu_{\tau}=\hbar^{2}|\mathbf{k}|^{2} / 2 \mu_{\tau}$ is the initial relative translational energy, and $\varepsilon_{\mathbf{n}}$ is the initial internal energy. (We occasionally denote the total energy by $E_{\text {tot }}$ for clarity.) The collision conserves total energy, which then defines a space (the "open channel space") of energetically accessible states in which reactants and products can be observed after the collision. These final states are labeled by $\mathbf{N}^{\prime} \tau^{\prime} \in$ open where $\mathbf{N}^{\prime}=\left(\mathbf{k}^{\prime}, \mathbf{n}^{\prime}\right)$ satisfies $E=\hbar^{2} k^{2} / 2 \mu_{\tau^{\prime}}$ $+\varepsilon_{\mathbf{n}^{\prime}}=\hbar^{2}\left|\mathbf{k}^{\prime}\right|^{2} / 2 \mu_{\tau^{\prime}}+\varepsilon_{\mathbf{n}^{\prime}}$. The scattering angles $\Omega$ define the rotation from the initial momentum direction $\mathbf{k} / k$ to the final momentum direction $\mathbf{k}^{\prime} / k^{\prime}$. With these definitions, the asymptotic form of the wavefunction is:

$$
\begin{aligned}
\lim _{R_{\tau^{\prime}} \rightarrow \infty} \Psi_{\mathbf{N} \tau}^{+}\left(\mathbf{R}_{\tau^{\prime}}, \mathbf{q}_{\tau^{\prime}}\right) & =\phi_{\mathbf{n} \tau^{\prime}}\left(\mathbf{q}_{\tau^{\prime}}\right) e^{i \mathbf{k} \cdot \mathbf{R}_{\tau^{\prime}}} \delta_{\tau^{\prime}, \tau} \\
& +\sum_{\mathbf{N}^{\prime} \tau^{\prime} \in \text { open }} \phi_{\mathbf{n}^{\prime} \tau^{\prime}}\left(\mathbf{q}_{\tau^{\prime}}\right) \frac{e^{i k^{\prime} R_{\tau^{\prime}}}}{R_{\tau^{\prime}}} f_{\mathbf{N}^{\prime} \tau^{\prime}, \mathbf{N} \tau^{\prime}}
\end{aligned}
$$

The first term is a plane wave of incoming reactants in channel $\mathbf{N} \tau$, and the second term is a sum of outgoing spherical waves in all open channels. The asymptotic scattering amplitude $f_{N^{\prime} \tau^{\prime}, N_{\tau}}$, which has units of length, can also be expressed as 
$f_{\mathbf{n}^{\prime} \tau^{\prime}, \mathbf{n} \tau}(v, \Omega)$. Evaluating the numerator and denomerator of Eq. (1.1) using quantum mechanical fluxes, the QRS differential reaction cross section is given by:

$$
d \sigma_{\mathbf{n}^{\prime} \tau^{\prime}, \mathbf{n} \tau}(v, \Omega) / d \Omega=\left|f_{\mathbf{n}^{\prime} \tau^{\prime}, \mathbf{n} \tau}(v, \Omega)\right|^{2}
$$

The task in QRS, then, is to compute the asymptotic scattering amplitudes given the system masses and the PES.

Before describing strategies for solving the QRS problem, we transform the scattering amplitude to simplify subsequent discussion. In particular, the scattering amplitude we compute in practice is an element of the unitary $S$-matrix for fixed total angular momentum $J$. This quantity is related to the usual scattering amplitude $f_{\mathbf{n}^{\prime} \tau^{\prime}, \mathbf{n} \tau}(v, \Omega)$ via $[13]$

$$
f_{\mathbf{n}^{\prime} \tau^{\prime}, \mathbf{n} \tau}(v, \Omega)=\frac{1}{2 i k} \sum_{J=0}^{\infty}(2 J+1) D_{K^{\prime}, K}^{J}(\Phi, \Theta, \Phi)\left[S_{\mathbf{n}^{\prime} \tau^{\prime}, \mathbf{n} \tau}^{J}(E)-\delta_{\mathbf{n}^{\prime}, \mathbf{n}} \delta_{\tau^{\prime}, \tau}\right],
$$

where $K, K^{\prime}$ are projection quantum numbers of total angular momentum along the initial and final propagation directions, respectively, and $D_{m^{\prime}, m}^{l}(\alpha, \beta, \gamma)$ is the Wigner rotation matrix (please see Chapter 5 for details). Since the $S$-matrix is unitary, we may interpret the square moduli of its elements as probabilities. We define

$$
\begin{aligned}
P_{\mathbf{n}^{\prime} \tau^{\prime}, \mathbf{n} \tau}^{J}(E) & \equiv\left|S_{\mathbf{n}^{\prime} \tau^{\prime}, \mathbf{n} \tau}^{J}(E)\right|^{2} \quad \text { is the state-to-state reaction probability }\left(\tau^{\prime} \neq \tau\right) \\
P_{\mathbf{n} \tau}^{J}(E) & \equiv \sum_{\mathbf{n}^{\prime} \tau^{\prime}} P_{\mathbf{n}^{\prime} \tau^{\prime}, \mathbf{n} \tau}^{J}(E) \quad \text { is the initial state selected reaction probability } \\
N^{J}(E) & \equiv \sum_{\mathbf{n}} P_{\mathbf{n} \tau}^{J}(E) \quad \text { is the cumulative reaction probability. }
\end{aligned}
$$

$\left[N^{J}(E)\right.$ can exceed unity and thus is not strictly a probability.] These quantities take on great importance in the subsequent discussion. We now survey traditional and modern computational techniques for solving the QRS problem.

\subsection{Theoretical Practices Old and New}

The QRS problem poses unique challenges which make otherwise useful quantum mechanical methods fail. This difficulty arises from the multi-arrangement nature of the reactive collision event (please see Fig. 1.1). For example, although 
the self consistent field (SCF) approximation gives qualitativsly correct results for electronic energies [14], an SCF reactive scattering wavefunction fails miserably because translation and vibration exchange roles from one arrangement to the other, as shown in Fig. 1.1. In addition, perturbation theory [i.e. the distorted wave Born approximation (DWBA)] is accurate only for uninteresting energy regimes [15], and requires the calculation of a relatively sophisticated inelastically distorted reference system [16]. Thus, the QRS problem is recalcitrant to a simple solution.

Solving the QRS problem is also qualitatively more difficult than the single arrangement, i.e. inelastic scattering calculation. In this simpler case, we may use a single coordinate system $(\mathbf{R}, \mathbf{q})$ and asymptotic basis $\left\{\phi_{\mathbf{n}}(\mathbf{q})\right\}$ to represent the scattering wavefunction, which facilitates the well known coupled channel (CC) expansion [17]. The radial dependence of the scattering wavefunction is determined by approximating the second derivative on a grid, using the resulting recursion relation to propagate the $\mathrm{CC}$ equations from small interparticle separations. Propagation to large interparticle separations gives the scattering amplitudes coupling all open channels for a given total energy. This is probably the most traditional approach of calculating numerically exact multichannel scattering amplitudes. A generalization of the inelastic CC calculation to the reactive case was proposed by Miller [18], wherein a simultaneous basis set expansion in all chemical arrangement Jacobi coordinates defines the $\mathrm{CC}$ space. Unfortunately, the reactive couplings give an effective nonlocal exchange interaction which precludes direct propagation of the reactive $\mathrm{CC}$ equations. Thus, the simultaneous use of several coordinate systems within the CC expansion does not lead to a tractable calculation; hence solving the QRS problem requires either developing a more general coordinate system, or rethinking the $\mathrm{CC}$ scheme.

The first accurate cross section calculation, performed by Schatz and Kuppermann [19] in 1976 on the $\mathrm{H}+\mathrm{H}_{2}$ reaction, involved a sufficiently complicated variant of $\mathrm{CC}$ propagation that its successful implementation was restricted to $\mathrm{H}+\mathrm{H}_{2}$ at low energies (i.e. no vibrational excitation). Nevertheless, this work represented a breakthrough in QRS. Indeed, one may view as an outgrowth of this work a general coordinate system which allows straightforward reactive $\mathrm{CC}$ calculations. This coor- 


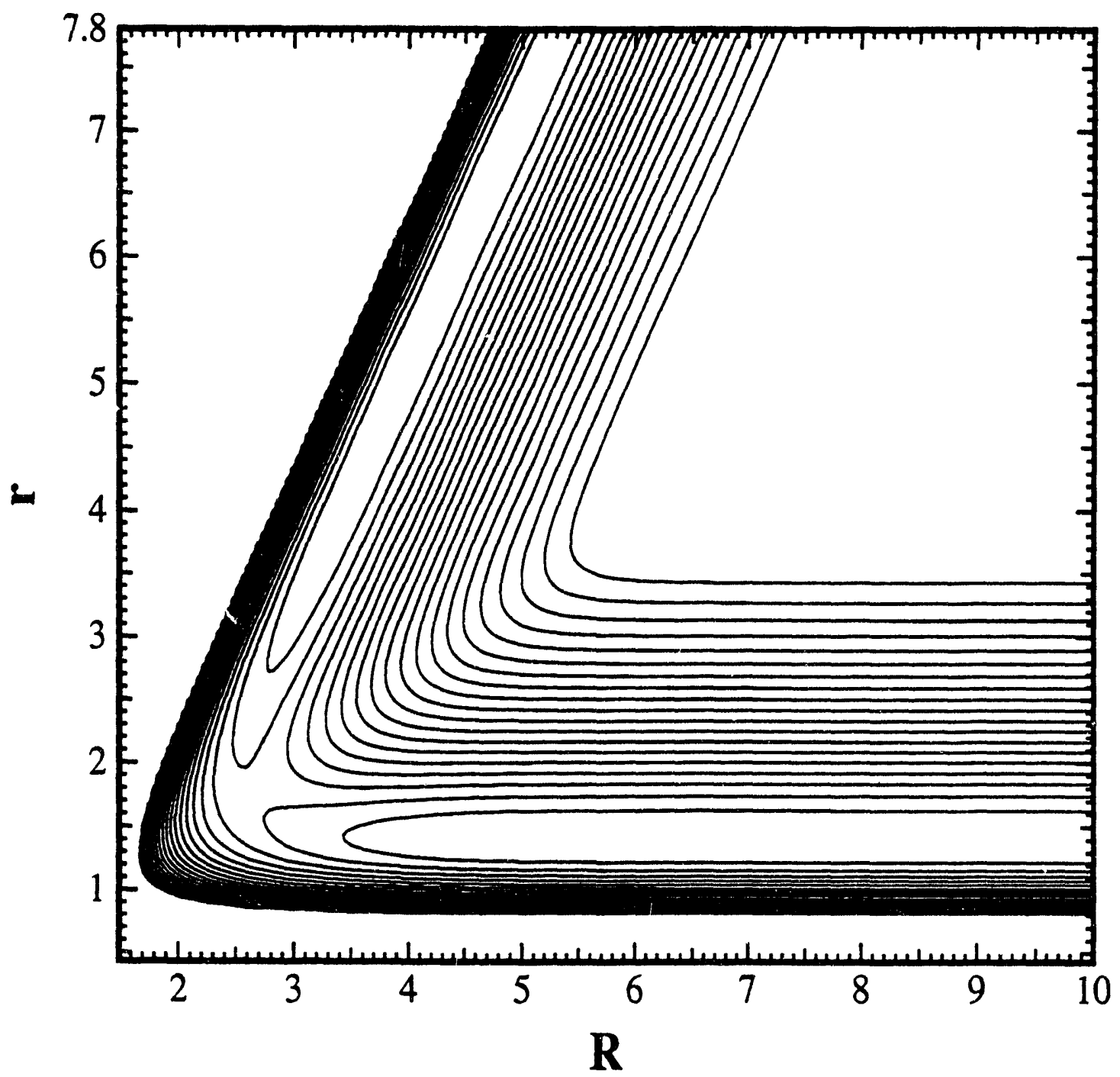

Figure 1.1: Contour plot of the Liu-Siegbahn-Truhlar-Horowitz potential energy surface for the $\mathrm{H}+\mathrm{H}_{2}$ reaction. The coordinate $B$ is the reactant scattering coordinate, and $r$ is the reactant vibrational coordinate. The portion for large $R$ is the reactant entrance valley, the portion with both $R$ and $r$ small is the reaction barrier region, and the portion for large $r$ is the product exit valley. We note that the scattering and vibrational coordinates approximately exchange roles upon :eaction. This strong coupling presents difficulty to otherwise useful quantum mechanical methods. 
dinate system, called hyperspherical coordinates $[20,21]$, uses a "vibrational angle" to smoothly vary from one chemical arrangement to another. The hyperspherical coordinate propagation (HSP) method has seen the most general use by application theorists, and hence may be considered the GAUSSIAN of QRS. A technical diffculty with HSP is that, unless the system masses satisfy certain criteria, the basis set must span vast regions of unimportant space. Indeed, HSP has been applied most frequently to heavy-light-heavy atom-diatom reactions. For this reason, various groups continued to search for more flexible solutions to the QRS problem.

A flurry of activity began in 1986 using Miller's 1969 formulation [18] when theorists realized how to use scattering variational principles [22-24] reliably to compute the radial function. As a result, accurate cross section calculations were reported for the $\mathrm{H}+\mathrm{H}_{2}$ reaction and its isotopic analogues, in addition to the important $\mathrm{F}+\mathrm{H}_{2}$ system, all over a wide range of energies [25]. We perform sensitivity analysis of $\mathrm{H}+\mathrm{H}_{2}$ scattering resonances in Chapter 2 using the $S$-matrix version of the Kohn variational principle [22]. We discuss briefly the philosophy behind this approach.

Since the use of multiple arrangement coordinates precludes $\mathrm{CC}$ propagation, the radial function must be expanded in a basis set. The variational principle simply gives a prescription for choosing the expansion coefficients, in addition to the helpful property that the variational $S$-matrix elements are invariant to first order error in the scattering wavefunction. In general, to expand the scattering wavefunction in a radial basis set requires the use of both $L^{2}$ basis functions and extended basis functions. The $L^{2}$ basis functions provide the flexibility to represent the wavefunction in the interaction region, whereas the extended functions enforce the asymptotic boundary conditions. Solving the Schrödinger equation with enforced scattering boundary conditions is tantamount to solving the corresponding integral equation with a Green's function [26]. Indeed, we demonstrate in Chapter 2 that the Kohn variational $S$-matrix can be expressed in terms of a variational approximation to the scattering Green's function [27]. The success of these variational calculations suggests that the Green's function approach to QRS, in addition to its formal utility, is an important computational tool. We pursue this idea in Chapters $3-5$ by developing efficient integral equation techniques for QRS. 
Until now we have discussed only time independent techniques for solving the QRS problem. This has long been the traditional approach. In the last few years, though, there has been tremendous progress in solving the time dependent Schrödinger equation $[28,29]$. Time dependent wavepacket propagation is now a well established method for solving the QRS problem, able to handle challenging reactions such as the $\mathrm{F}+\mathrm{H}_{2}$ system [30]. In fact, we borrow ideas from the time dependent approach throughout this dissertation.

As we have shown, several approaches for exact reactive scattering calculations are currently available. By construction, all of those discussed involve determining the state-to-state scattering amplitudes. In extending exact theory to larger systems (i.e. four or more atoms), it may not be appropriate (or possible) to study chemical reactions in such detail. Indeed, a theoretical framework based on the direct calculation of averaged reaction probabilties should be more applicable to larger systems. Transition state theory provides a very useful, albeit approximate framework for calculating averaged reaction probabilities [31]. To quote a 1976 review article [32] on "quantum transition state theory," we wish ...

... to use the fundamental assumption of transition state theory to simplify quantum mechanical scattering calculations by imposing boundary conditions on the scattering equations that take advantage of the "direct" nature of dynamics in the saddle point region.

In Chapters 3-5, we develop and apply a new Green's function formulation of QRS which fulfills this 17 year old wish. We augment the physical Hamiltonian with a negative imaginary potential to absorb outgoing flux emanating from the saddle point region. The absorbing boundary condition $(A B C)$ formulation of QRS allows the direct calculation of the cumulative, initial state selected, and state-to-state reaction probabilities, with concomitant amounts of computational effort.

The ABC method reduces the scattering problem to the determination of the $A B C$ Green's function $(G)$, a matrix inverse. Although formally this is just as computationally demanding as full diagonalization, the initial state selected formalism only requires a single column of $\mathrm{G}$. Iterative methods, which require very little core memory, can be used to compute a single column of $\mathbf{G}$ rapidly. We develop an iterative 
method especially suited for the calculation of $\mathrm{G}$ in Chapters 3 and 4 , which evaluates $G$ as the half-Fourier transform of the propagator. As we will show in Chapter 5, we are able to converge initial state selected reaction cross sections for $\mathrm{D}+\mathrm{H}_{2}$ using the new method in 10 minutes on an IBM RS/6000.

\subsection{Looking Ahead}

We use in Chapter 2 the Kohn variational $S$-matrix formalism to probe the sensitivity of $\mathrm{H}+\mathrm{H}_{2}$ cross sections to small changes in the PES, to help resolve a discrepancy between experiment and theory over a possible $\mathrm{H}_{3}$ collision complex. We find the reactive scattering calculations to be very robust, and thus trust their predictions.

We develop in Chapter 3 a time dependent calculation of the ABC Green's function on a grid, called the power series Green's function (PSG). We compute the cumulative reaction probability for the collinear $\mathrm{H}+\mathrm{H}_{2}$ test problem. The similarity of our approach in Chapter 3 to modern path integral methods is also discussed.

We discuss thoroughly in Chapter 4 the feasibility of time independent approaches for computing the ABC Green's function. We eventually come full circle, in the end developing a technique called the Newton algorithm similar in spirit to the PSG, but vastly improved in efficiency. We test the Newton algorithm on the calculation of initial state selected reaction probabilities for the three dimensional $\mathrm{D}+\mathrm{H}_{2}$ reaction, and find both rapid convergence and strict accuracy control.

We apply in Chapter 5 the ABC-Newton machinery to calculating the $\mathrm{D}+\mathrm{H}_{2}$ $(v=1, j)$ initial state selected cross sections and rate constants. We find remarkably rapid convergence of the quantum calculations, and for the first time obtain quantitative agreement with experiment for the initial vibrationally excited rate constant $k_{v=1}(T=310 K)$.

Finally, we conclude the dissertation in Chapter 6 with a summary of the research reported, and with suggestions for future work. 


\section{References}

[1] H. Primas, Chemistry, Quantum Mechanics, and Reductionism (Springer Verlag, Berlin, 1983).

[2] Aristotle, Physics, in Aristotle: Selected Works, Third Edition, translated by H. G. Apostle and L. P. Gerson (The Peripatetic Press, Grinnell, Iowa, 1991), pp. 169-240.

[3] Aristotle, History of Animals, Parts of Animals, and Generation of Animals, in Aristotle: Selected Works, Third Edition, translated by H. G. Apostle and L. P. Gerson (The Peripatetic Press, Grinnell, Iowa, 1991), pp. 301-330.

[4] M. Marchi, J. N. Gehlen, D. Chandler, and M. Newton, J. Am. Chem. Soc. 115, 4178 (1993).

[5] Early work in molecular beams can be found in Molecular Beams, ed. J. Ross, in Adv. Chem. Phys. 10, 1 (1966).

[6] For a recent review of molecular beam scattering in chemistry, see Y. T. Lee, Science 236, 793 (1987).

[7] R. D. Levine and R. B. Bernstein, Molecular Molecular Reaction Dynamics and Chemical Reactivity (Oxford University Press, New York, 1987).

[8] J. C. Nieh and J. J. Valentini, Phys. Rev. Lett. 60, 519 (1988).

[9] M. L. Goldberger and K. M. Watson, Collision Theory (Wiley, New York, 1964).

[10] L. S. Rodberg and R. M. Thaler, Introduction to the Quantum Theory of Scattering (Academic Press, New York, 1967).

[11] R. G. Newton, Scattering Theory of Waves and Particles, Second Edition (Springer-Verlag, Heidelberg, 1982).

[12] J. N. Murrell, S. Carter, S. C. Farantos, P. Huxley, and A. J. C. Varandas, Molecular Potential Energy Functions (Wiley, New York, 1984). 
[13] M. Jacob and G. C. Wick, Ann. Phys. 7, 404 (1959).

[14] D. A. McQuarrie, Quantum Chemistry (University Science Books, Mill Valley, CA, 1983), pg. 295.

[15] The DWBA is accurate when reaction cross sections are very small. For all scattering systems, this obtains for uninterestingly large initial translational energies. For systems with a barrier to reaction, the DWBA may also be accurate for very small initial translational energies. The DWBA tends to overestimate cross sections in the more interesting intermediate energy regime.

[16] L. M. Hubbard, S. Shi, and W. H. Miller, J. Chem. Phys. 78, 2381 (1983).

[17] W. A. Lester, Dynamics of Molecular Collisions, Part A, ed. W. H. Miller (Plenum, New York, 1976), pp. 1-32.

[18] W. H. Miller, J. Chem. Phys. 50, 407 (1969).

[19] G. C. Schatz and A. Kuppermann, J. Chem. Phys. 65, 4642, 4668 (1976).

[20] A. Kuppermann and P. G. Hipes, J. Chem. Phys. 84, 5962 (1986).

[21] R. T. Pack and G. A. Parker, J. Chem. Phys. 87, 3888 (1987).

[22] J. Z. H. Zhang, S.-I. Chu, and W. H. Miller, J. Chem. Phys. 88, 6233 (1988).

[23] D. E. Manolopoulos and R. E. Wyatt, J. Chem. Phys. 91, 6096 (1989).

[24] K. Haug, D. W. Schwenke, Y. Shima, D. G. Truhlar, J. Z. H. Zhang, and D. J. Kouri, J. Phys. Chem. 90, 6757 (1986).

[25] For a recent review of this work, see W. H. Miller, Annu. Rev. Phys. Chem. 41, 245 (1990).

[26] E. N. Economou, Green's Functions in Quantum Physics (Springer, Berlin, 1990). 
[27] W. H. Miller and B. M. D. D. Jansen op de Haar, J. Chem. Phys. 86, 6213 (1987).

[28] For a review of time dependent calculations, see

R. Kosloff, J. Phys. Chem. 92, 2087 (1988).

[29] For a compendium of recent work in time dependent quantum mechanics, see Time Dependent Methods for Quantum Dynamics, ed. K. C. Kulander, in Comput. Phys. Commun. 63, 1 (1991).

[30] D. Neuhauser, R. S. Judson, R. L. Jaffe, M. Baer, and D. J. Kouri, Chem. Phys. Lett. 176, 546 (1991).

[31] P. Pechukas, Dynamics of Molecular Collisions, Part B, ed. W. H. Miller (Plenum, New York, 1976), pp. 269-322.

[32] W. H. Miller, Acc. Chem. Res. 9, 306 (1976). 


\section{Chapter 2}

\section{Sensitivity Analysis for $\mathbf{H}+\mathbf{H}_{2}$}

\subsection{Introduction}

The last few years have seen dramatic advances in the rigorous theoretical description of elementary chemical reactions, i.e. in exact quantum mechanical reactive scattering calculations [1-6]. These theoretical developments are especially timely because of parallel advances in experimental studies of elementary reactions [7-12]. Several groups have reported new studies of the $\mathrm{H}+\mathrm{H}_{2}, \mathrm{D}+\mathrm{H}_{2}$, and $\mathrm{H}+\mathrm{D}_{2}$ reactions, and this makes possible comparisons of unprecedented detail between theory and experiment. Most intriguing are the integral cross section results of Nieh and Valentini $[10,11]$ for the reaction

$$
\mathrm{H}+\text { para }-\mathrm{H}_{2}(v=0, j=0) \rightarrow \text { ortho- } \mathrm{H}_{2}\left(v^{\prime}=1, j^{\prime}=1,3\right)+\mathrm{H}
$$

which show significant discrepancies with the theoretical results [13]. Figure 2.1, for example, clearly shows that for the $(v=0, j=0)$ to $\left(v^{\prime}=1, j^{\prime}=1\right)$ transition, Nieh and Valentini report a prominent scattering resonance at $1.2 \mathrm{eV}$, in significant disagreement with the theoretical results. The experimental findings are the first of their kind, and therefore are in need of confirmation. The theoretical results, on the other hand, have been confirmed independently by two other groups [14, 15] and have been performed on what is thought to be a very accurate potential energy surface (PES) for this reaction - the Liu-Siegbahn-Truhlar-Horowitz [16, 17] (LSTH) 
PES. Nevertheless, the discrepancies are so large that they may indicate anomalous sensitivity in the quantum calculations to the accuracy of the PES. The goal of the present Chapter is thus to begin to analyze our reactive scattering calculations for such extreme sensitivity to features in the PES.

A general goal of such analysis, generically referred to as sensitivity analysis [18], is to relate features in the PES to the resulting dynamical properties. To this end, several general methods may be considered. A simple type of sensitivity analysis involves brute force comparisons, in which dynamical calculations are carried out with different potentials [19-22]. One then relates the difference between the dynamical results (e.g. state-to-state cross sections) to the difference between the potentials (e.g. near the barrier heights) in order to characterize the sensitivity. The brute force method has the benefit that the full effect on the dynamics from changing the potential can be observed. However, this sensitivity is determined by the particular PESs chosen for study, and is therefore a function only of those potentials. A complementary method is functional sensitivity analysis [23] in which the effect on the dynamics of a first order functional variation to a reference PES is studied. The sensitivity is approximated by the first order functional derivative of the cross section with respect to the PES. Since this sensitivity function depends upon molecular coordinates, it indicates (to first order) which molecular configurations are most important for a given dynamical process.

In the present Chapter, we begin to probe the sensitivity of the quantum scattering calculations to the accuracy of the PES using a brute force comparison. In particular, we wish to know how a small change in the PES near the barrier will affect the integral cross sections for the transitions in Fig. 2.1. To determine this, we have calculated representative partial cross sections for the $(v=0, j=0)$ to $\left(v^{\prime}=1, j^{\prime}=1,3\right)$ transitions over a wide range of energies using the LSTH and double many body expansion [24] (DMBE) ab initio PESs. The DMBE PES is an interesting surface for comparison because, for the present purposes, it differs from the LSTH PES most significantly in the barrier height regions of both collinear and noncollinear $\mathrm{H}_{3}$ configurations. The quantitative details of the difference between the two PESs are discussed below. The calculations were carried out using the $S$-matrix 


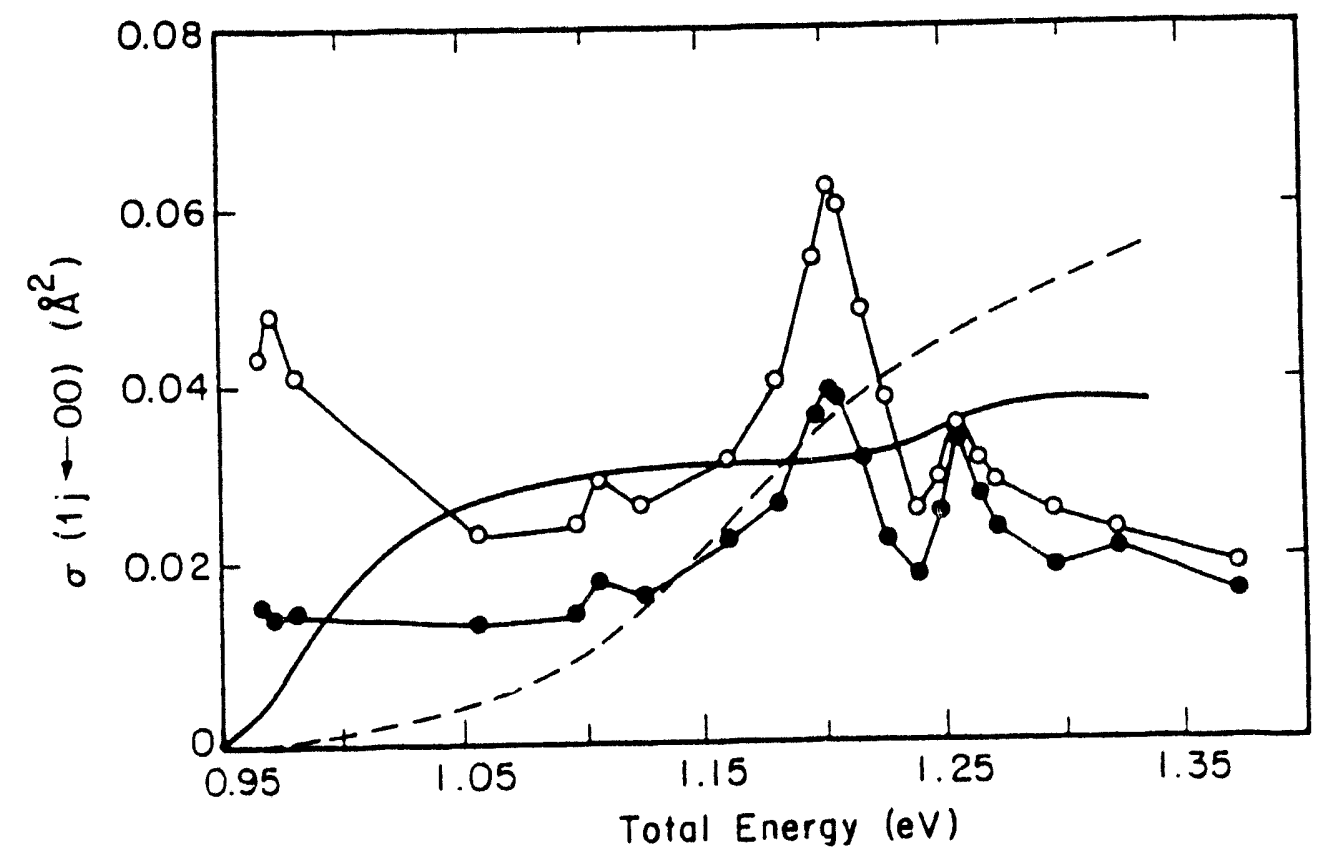

Figure 2.1: Experimental and theoretical integral cross sections for the $\mathrm{H}+\mathrm{H}_{2}$ transitions $(v=0, j=0)$ to $\left(v^{\prime}=1, j^{\prime}=1,3\right)$ in reaction $(2.1)$ as a function of total energy. The solid curve and open points are for the final state $\left(v^{\prime}=1, j^{\prime}=1\right)$, and the broken curve and solid points for final state $\left(v^{\prime}=1, j^{\prime}=3\right)$. The experiment measured absolute cross sections, for which there is good agreement with theory. However, the sharp features in the experimental energy dependence are not matched by theory. 
Kohn variational method that has been described in detail in previous publications [5, $25,26]$. We briefly review this approach for quantum reactive scattering below [27]. In addition, we demonstrate how to apply functional sensitivity analysis within the $S$-matrix Kohn framework. We report the cross section calculations resulting from the two PESs for total angular momenta $J=0$ and 10. We find that the theoretical cross sections do not change significantly when the LSTH PES is replaced by the DMBE [28]. This suggests that the theoretical prediction - that there is no sharp resonance in the $\mathrm{H}+\mathrm{H}_{2}$ integral cross section - may be correct.

\section{$2.2 S$-matrix Kohn Formulation}

We introduce the $S$-matrix version of the Kohn variational principle (KVP) for a reactive system with chemical arrangements labeled hy $\tau$. For each arrangement we have a radial scattering coordinate $R_{\tau}$ and internal coordinates $\mathbf{q}_{\tau}$. A simple way to start is by writing a reactive $S$-matrix element in the distorted Born representation:

$$
\begin{aligned}
S_{\mathbf{n}^{\prime} \tau^{\prime}, \mathbf{n} \tau}(E) & =\tilde{S}_{\mathbf{n}^{\prime} \tau^{\prime}, \mathbf{n} \tau}(E) \\
& +\frac{i}{\hbar}\left\langle\tilde{\psi}_{\mathbf{n}^{\prime} \tau^{\prime}}^{-}|\hat{H}-E| \tilde{\psi}_{\mathbf{n} \tau}^{+}\right\rangle \\
& +\frac{i}{\hbar}\left\langle(\hat{H}-E) \tilde{\psi}_{\mathbf{n}^{\prime} \tau^{\prime}}^{-}\left|\hat{G}^{+}(E)\right|(\hat{H}-E) \tilde{\psi}_{\mathbf{n} \tau}^{+}\right\rangle
\end{aligned}
$$

In Eq. (2.2), $\hat{G}^{+}(E) \equiv \lim _{\epsilon \rightarrow 0^{+}}(E+i \epsilon-\hat{H})^{-1}$ is the scattering Green's function with outgoing wave (i.e. " + ") boundary conditions. Also, $\tilde{\psi}_{\mathbf{n} \tau}^{+}\left(R_{\tau}, \mathbf{q}_{\tau}\right)$ is a trial wavefunction which can be anything from a free wave to the exact reactive scattering wavefunction, as long as it has the following boundary conditions:

$$
\begin{aligned}
\lim _{R_{\tau} \rightarrow 0^{+}} \tilde{\psi}_{\mathbf{n} \tau}^{+}\left(R_{\tau}, \mathbf{q}_{\tau}\right) & =0 \\
\lim _{R_{\tau} \rightarrow \infty} \tilde{\psi}_{\mathbf{n} \tau}^{+}\left(R_{\tau}, \mathbf{q}_{\tau}\right) & =\sum_{\mathbf{n}^{\prime} \tau^{\prime} \in \text { open }} \frac{\phi_{\mathbf{n}^{\prime} \tau^{\prime}}\left(\mathbf{q}_{\tau}^{\prime}\right)}{\sqrt{v_{\mathbf{n}^{\prime} \tau^{\prime}}}} \\
& \times\left\{-e^{-i k_{\mathbf{n}^{\prime} \tau^{\prime}} R_{\tau^{\prime}} \delta_{\mathbf{n}^{\prime}, \mathbf{n}}} \delta_{\tau^{\prime}, \tau}+e^{i k_{\mathbf{n}^{\prime} \tau^{\prime}} R_{\tau^{\prime}}} \tilde{S}_{\mathbf{n}^{\prime} \tau^{\prime}, \mathbf{n} \tau}(E)\right\},
\end{aligned}
$$

where $\tilde{S}_{\mathbf{n}^{\prime} \tau^{\prime}, \mathbf{n} \tau}(E)$ is a unitary reference $S$-matrix. That is, $\tilde{S}_{\mathbf{n}^{\prime} \tau^{\prime}, \mathbf{n} \tau}(E)$ is the reference asymptotic amplitude associated with the trial wavefunction $\tilde{\psi}_{\mathbf{n} \tau}^{+}\left(R_{\tau}, \mathbf{q}_{\tau}\right)$. In Eq. 
(2.3), $\phi_{\mathbf{n} \tau}\left(\mathbf{q}_{\tau}\right)$ is an eigenfuction for the internal motions of the system in arrangement $\tau, v_{\mathbf{n} \tau}$ is the asymptotic translational velocity for total energy $E$ in channel $\mathbf{n} \tau$, and the sum is over all open channels at total energy $E$. If $\tilde{\psi}_{\mathbf{n} \tau}^{+}\left(R_{\tau}, \mathbf{q}_{\tau}\right)$ is exact, Eq. (2.2) trivially gives the exact $S$-matrix, since $\tilde{S}_{\mathbf{n}^{\prime} \tau^{\prime}, \mathbf{n} r}(E)$ is exact and $(\hat{H}$ $E)\left|\tilde{\psi}_{\mathbf{n} \tau}^{+}\right\rangle$vanishes. In general, as long as $\hat{G}^{+}(E)$ is exact and $\tilde{\psi}_{\mathbf{n} r}^{+}\left(R_{\tau}, \mathbf{q}_{\tau}\right)$ satisfies the boundary conditions in Eq. (2.3) with $\tilde{S}_{\mathbf{n}^{\prime} \tau^{\prime}, \mathbf{n} \tau}(E)$ being unitary, the distorted Born representation of $S_{\mathbf{n}^{\prime} \tau^{\prime}, \mathbf{n} \tau}(E)$ is exact regardless of the quality of the reference system.

The computational challenge in quantum scattering theory can be discussed in terms of Eq. (2.2). As mentioned above, the trial wavefunction can be anything from a free wave to the exact scattering wavefunction. If one devotes the computational effort to make $\tilde{\psi}_{\mathbf{n} \tau}^{+}\left(R_{\tau}, \mathbf{q}_{\tau}\right)$ exact, then no effort is required to evaluate the Green's function in the third term in Eq. (2.2), since that term does not contribute. Alternatively, if $\tilde{\psi}_{\mathbf{n} \tau}^{+}\left(R_{\tau}, \mathbf{q}_{\tau}\right)$ is a free wave, i.e. $\tilde{S}_{\mathbf{n}^{\prime} \tau^{\prime}, \mathbf{n} \tau}(E)=0$ or $\tilde{S}_{\mathbf{n}^{\prime} \tau^{\prime}, \mathbf{n} \tau}(E)=$ $\delta_{\mathbf{n}^{\prime}, \mathbf{n}} \delta_{\tau^{\prime}, \tau}$, the Green's function is required over a relatively large region of space. An intermediate case, which may be the most practical, is where a partially distorted wave and the Green's function over a small region are used to obtain the $S$-matrix. We will pursue the free wave - Green's function approach later in this dissertation.

The philosophy behind the KVP is to variationally optimize $\tilde{\psi}_{\mathbf{n} \tau}^{+}\left(R_{\tau}, \mathbf{q}_{\tau}\right)$ based on making the first two terms in Eq. (2.2) stationary, and neglecting the Green's function term. In addition to giving a prescription for obtaining $\tilde{\psi}_{\mathbf{n}_{\tau}}^{+}\left(R_{\tau}, \mathbf{q}_{\tau}\right)$, this gives an approximate $S$-matrix which is invariant to first order error in $\tilde{\psi}_{\mathbf{n} \tau}^{+}\left(R_{\tau}, \mathrm{q}_{\tau}\right)$. The KVP gives

$$
S_{\mathbf{n}^{\prime} \tau^{\prime}, \mathbf{n} \tau}^{K V P}(E)=\operatorname{ext}\left[\tilde{S}_{\mathbf{n}^{\prime} \tau^{\prime}, \mathbf{n} \tau}(E)+\frac{i}{\hbar}\left\langle\tilde{\psi}_{\mathbf{n}^{\prime} \tau^{\prime}}^{-}|\hat{H}-E| \tilde{\psi}_{\mathbf{n} \tau}^{+}\right\rangle\right]
$$

where "ext" means extremize (not minimize because $S_{\mathbf{n}^{\prime} \tau^{\prime}, \mathbf{n} \tau}^{K V P}(E)$ is complex) with respect to first order variations in $\tilde{\psi}_{\mathbf{n} r}^{+}\left(R_{\tau}, \mathbf{q}_{\tau}\right)$. To apply the extremum condition, we assume an ansatz for $\tilde{\psi}_{\mathbf{n} \tau}^{+}\left(R_{\tau}, \mathbf{q}_{\tau}\right)$ with some variational parameters. Although in general these may be nonlinear, we will use only linear variational parameters to simplify the resulting algebraic equations. The KVP wavefunction is thus

$$
\tilde{\psi}_{\mathbf{n} \tau}^{+}\left(R_{\tau}, \mathbf{q}_{\tau}\right)=\sum_{\mathbf{n}^{\prime} \tau^{\prime} \in \text { open }} \frac{\phi_{\mathbf{n}^{\prime} \tau^{\prime}}\left(\mathbf{q}_{\tau}^{\prime}\right)}{\sqrt{v_{\mathbf{n}^{\prime} \tau^{\prime}}}}\left[-u_{0 \mathbf{n}^{\prime} \tau^{\prime}}\left(R_{\tau^{\prime}}\right) \delta_{\mathbf{n}^{\prime}, \mathbf{n}} \delta_{\tau^{\prime}, \tau}+u_{1 \mathbf{n}^{\prime} \tau^{\prime}}\left(R_{\tau^{\prime}}\right) c_{1 \mathbf{n}^{\prime} \tau^{\prime}, \mathbf{n} \tau}\right]
$$




$$
+\sum_{\mathbf{n}^{\prime} \tau^{\prime} \in \text { opdl }} \sum_{t^{\prime}=2}^{N} \frac{\phi_{\mathbf{n}^{\prime} \tau^{\prime}}\left(\mathbf{q}_{\tau}^{\prime}\right)}{\sqrt{v_{\mathbf{n}^{\prime} \tau^{\prime}}}} u_{t^{\prime} \mathbf{n}^{\prime} \tau^{\prime}}\left(R_{\tau^{\prime}}\right) c_{t^{\prime} \mathbf{n}^{\prime} \tau^{\prime}, \mathbf{n} \tau},
$$

where $\left\{c_{t^{\prime} \mathbf{n}^{\prime} \tau^{\prime}, \mathbf{n} \tau}\right\}$ for $t^{\prime}=1, \ldots, N$ are the linear variational coefficients. The $u_{0}$ term provides incoming wave boundary conditions in channel $\mathbf{n} \tau$, the $u_{1}$ term gives outgoing wave boundary conditions in all energetically accessible channels, and the $u_{t}$ for $t=2, \ldots, N$ terms (which vanish asymptotically) provide the flexibility to represent the wavefunction in the interaction region. We note that, with the present notation, $\tilde{S}_{\mathbf{n}^{\prime} \tau^{\prime}, \mathbf{n} \tau}(E)=c_{1 \mathbf{n}^{\prime} \tau^{\prime}, \mathbf{n} \tau}$. The first channel sum in Eq. (2.5) is over only open channels (open) because this part of the wavefunction is meant to satisfy the asymptotic boundary conditions in Eq. (2.3). In addition, the channel space in this first sum defines the active index space for the KVP $S$-matrix. The second channel sum in Eq. (2.5) is over open and closed channels (opcl), because this part of the wavefunction is just a basis set expansion. Furthermore, we note that it is not necessary to use the asymptotic eigenfunctions to represent the internal motions in the interaction region. Indeed, in the remainder of this dissertation we pursue the use of grid methods to represent all degrees of freedom in the interaction region. The calculations presented in this Chapter did, however, use the asymptotic eigenfunction basis.

Using this trial wavefunction in the Kohn variational expression yields the following stationary $S$-matrix element:

$$
S_{\mathbf{n}^{\prime} \tau^{\prime}, \mathbf{n} \tau}^{K V P}(E)=\frac{i}{\hbar}\left[\mathbf{M}_{00}-\mathbf{M}_{0}^{T} \cdot \mathbf{M}^{-1} \cdot \mathbf{M}_{0}\right]_{\mathbf{n}^{\prime} \tau^{\prime}, \mathbf{n} \tau}
$$

where

$$
\begin{aligned}
& {\left[\mathbf{M}_{00}\right]_{\mathbf{n}^{\prime} \tau^{\prime}, \mathbf{n} \tau}=\left\langle u_{0 \mathbf{n}^{\prime} \tau^{\prime}} \phi_{\mathbf{n}^{\prime} \tau^{\prime}}|\hat{H}-E| u_{0 \mathbf{n} \tau} \phi_{\mathbf{n} \tau}\right\rangle } \\
& {\left[\mathbf{M}_{0}\right]_{t^{\prime} \mathbf{n}^{\prime} \tau^{\prime}, \mathbf{n} \tau}=\left\langle u_{t^{\prime} \mathbf{n}^{\prime} \tau^{\prime}} \phi_{\mathbf{n}^{\prime} \tau^{\prime}}|\hat{H}-E| u_{0 \mathbf{n} \tau} \phi_{\mathbf{n} \tau}\right\rangle \quad t^{\prime}=1, \ldots, N } \\
& {[\mathbf{M}]_{t^{\prime} \mathbf{n}^{\prime} \tau^{\prime}, t \mathbf{n} \tau}=\left\langle u_{t^{\prime} \mathbf{n}^{\prime} \tau^{\prime}} \phi_{\mathbf{n}^{\prime} \tau^{\prime}}|\hat{H}-E| u_{t \mathbf{n} \tau} \phi_{\mathbf{n} \tau}\right\rangle \quad t^{\prime}, t=1, \ldots, N . }
\end{aligned}
$$

We note that the incoming wave boundary conditions in the bra state in Eqs. (2.2) and (2.4) can be enforced by not complex conjugating radial functions in bra states of Eq. (2.7). This inner product is called the biorthogonal inner product [29], and is formally related to the use of complex scaled coordinates and absorbing boundary conditions. 
As we have shown, the KVP reduces the quantum scattering problem to choosing basis functions, computing matrix elements of the Hamiltonian, and performing a linear algebra calculation to obtain $\mathbf{M}^{-1} \cdot \mathbf{M}_{0}$. Before proceeding with the discussion of sensitivity analysis, we make some qualitative remarks concerning the KVP.

The KVP resembles the Rayleigh-Ritz variational principle [30] (RRVP). Indeed, if the scattering variational formulation were applied to a bound state problem, for which $S_{\mathbf{n}^{\prime} \tau^{\prime}, \mathbf{n} \tau}^{K V P}(E)=0=\tilde{S}_{\mathbf{n}^{\prime} \tau^{\prime}, \mathbf{n} \tau}(E)$, the KVP reduces to

$$
0=\operatorname{ext}\left[\left\langle\tilde{\psi}_{E}|\hat{H}-E| \tilde{\psi}_{E}\right\rangle\right] \text {. }
$$

This is precisely the RRVP. As such, the KVP can be seen as the prope $e_{1}$ generalization of the RRVP for case of scattering boundary conditions.

The KVP also looks similar to the distorted wave Born approximation [31] (DWBA), which is a perturbative approximation. That the KVP is capable of arbitrary accuracy can be seen from expressing the KVP $S$-matrix in a way which resembles Eq. (2.2). By performing integration by parts to rearrange the $\mathbf{M}_{0}^{T} \cdot \mathbf{M}^{-1} \cdot \mathbf{M}_{0}$ term, one can write the KVP $S$-matrix as:

$$
\begin{aligned}
S_{\mathbf{n}^{\prime} \tau^{\prime}, \mathbf{n} \tau}^{K V P}(E) & =c_{1 \mathbf{n}^{\prime} \tau^{\prime}, \mathbf{n} \tau} \\
& +\frac{i}{\hbar}\left\langle u_{0 \mathbf{n}^{\prime} \tau^{\prime}} \phi_{\mathbf{n}^{\prime} \tau^{\prime}}|\hat{H}-E| u_{0 \mathbf{n} \tau} \phi_{\mathbf{n} \tau}\right\rangle \\
& +\frac{i}{\hbar}\left\langle u_{0 \mathbf{n}^{\prime} \tau^{\prime}} \phi_{\mathbf{n}^{\prime} \tau^{\prime}}\left|(\hat{H}-E) \hat{G}^{K V P}(E)(\hat{H}-E)\right| u_{0 \mathbf{n} \tau} \phi_{\mathbf{n} \tau}\right\rangle,
\end{aligned}
$$

where the Kohn variational approximation to the Green's function is given by [32]

$$
\hat{G}^{K V P}(E)=-\sum_{t^{\prime}, t=1}^{N} \sum_{\mathbf{n}^{\prime} \tau^{\prime}, \mathbf{n} \tau \in \text { opdl }}\left|u_{t^{\prime} \mathbf{n}^{\prime} \tau^{\prime}} \phi_{\mathbf{n}^{\prime} \tau^{\prime}}\right\rangle\left[\mathbf{M}^{-1}\right]_{t^{\prime} \mathbf{n}^{\prime} \tau^{\prime}, t \mathbf{n} \tau}\left\langle u_{t \mathbf{n} \tau} \phi_{\mathbf{n} \tau}\right| .
$$

Since the sums over $t^{\prime}, t$ begin with $1, \hat{G}^{K V P}(E)$ has only outgoing asymptotic waves. Equations (2.4), (2.9), and (2.10) demonstrate the close relationship between perturbation theory and variational theory in quantum scattering. That is, one can view the KVP $S$-matrix as perturbation theory with a very good reference system, or as variational theory with a purely incoming, free wave reference state. Although it 
is not standard to use purely incoming wave reference scattering states, we use this approach in Chapters 4 and 5 inspired by its success in the KVP. We also note that as the basis set $\left\{u_{t \mathbf{n} \tau} \phi_{\mathbf{n} \tau}\right\}$ becomes complete, the second term identically cancels the third in Eq. (2.10), while $c_{1 \mathbf{n}^{\prime} \tau^{\prime}, \mathbf{n} \tau}$ approaches the exact $S$-matrix.

This completes our discussion of the KVP. In closing this Section, we note that although the KVP has made quantum reactive scattering calculations straightforward in principle, its implementation can be very computationally demanding in practice. As discussed in Chapter 4, this is due to the fact that the KVP Green's function requires explicit construction of all outgoing asymptotic waves. This level of detail may not be attainable in treating the reactivity of complex systems. For this reason, alternative methods are pursued in the subsequent Chapters of this dissertation.

\subsection{Functional Sensitivity Analysis}

Functional sensitivity analysis is an attempt to locate which regions of the PES are most important for determining particular dynamical events [18, 23], i.e. transitions from $\mathbf{n}^{\prime} \tau^{\prime}$ to $\mathbf{n} \tau$ at energy $E$. Some function of molecular coordinates, called the "sensitivity coefficient" (SC), is required to indicate which configurations are important. In general, this function is not known. An approximation to the $\mathrm{SC}$, however, is obtained from computing the first functional derivative of $S_{\mathbf{n}^{\prime} \tau^{\prime}, \mathbf{n} \tau}(E)$ with respect to $V(R, \mathbf{q}$ ) (we suppress arrangement channel indices on $(R, \mathbf{q})$ to avoid confusion, and suppose a particular coordinate system for the present discussion). The KVP SC is obtained by differentiating Eq. (2.4), giving

$$
\frac{\delta S_{\mathbf{n}^{\prime} \tau^{\prime}, \mathbf{n} \tau}(E)}{\delta V(R, \mathbf{q})}=\frac{i}{\hbar} \tilde{\psi}_{\mathbf{n}^{\prime} \tau^{\prime}}^{-}(R, \mathbf{q}) \tilde{\psi}_{\mathbf{n} r}^{+}(R, \mathbf{q})
$$

As such, once the scattering calculation is performed, the SC is easily constructed for a particular collision event. We see that the KVP SC places importance in regions where the scattering wavefunctions have large amplitude.

An interesting application of functional sensitivity analysis is in determining which portion of a one dimensional barrier potential is most important for tunneling 
[23]. One suspects that the region near top of the barrier will be most important. The width of the important region, however, is unclear. To determine this, we focus on the deep tunneling regime, for which the energy dependent tunneling probability $T(E)$ is well approximated by the Wentzel-Kramers-Brillouin result [33] (WKB), given by

$$
\begin{aligned}
T(E) & \cong e^{-2 \theta(E)} \\
\theta(E) & =\int_{x_{1}(E)}^{x_{2}(E)} \frac{d x}{\hbar} \sqrt{2 m[V(x)-E]}
\end{aligned}
$$

where $\left[x_{1}(E), x_{2}(E)\right]$ are the total energy and potential energy dependent classical turnirg points. The corresponding SC is given by

$$
\frac{\delta T(E)}{\delta V(x)}=-2 T(E) \times \frac{\delta \theta(E)}{\delta V(x)} .
$$

The functional derivative of $\theta(E)$ has three terms, because of the potential dependence of the turning points. Differentiation gives

$$
\begin{aligned}
\frac{\delta \theta(E)}{\delta V(x)} & =\frac{1}{\hbar} \sqrt{2 m\left[V\left(x_{2}\right)-E\right]} \frac{\delta x_{2}(E)}{\delta V(x)} \\
& -\frac{1}{\hbar} \sqrt{2 m\left[V\left(x_{1}\right)-E\right]} \frac{\delta x_{1}(E)}{\delta V(x)} \\
& +\frac{\sqrt{2 m}}{2 \hbar}[V(x)-E]^{-1 / 2}
\end{aligned}
$$

The first two terms in Eq. (2.14) vanish by the definition of turning points. The WKB $\mathrm{SC}$ thus becomes

$$
\frac{\delta T(E)}{\delta V(x)}=-\frac{2 T(E)}{\hbar v(x)}
$$

where $v(x)=\sqrt{2[V(x)-E] / m}$ is the magnitude of the imaginary velocity in the tunneling region. Equation (2.15) indicates that the WKB SC is largest (i.e. blows $\mathrm{up}$ ) at the turning points. The exact quantum $\mathrm{SC}$, which is finite for all $x$ [cf. Eq. (2.11)], is also largest at the classical turning points. Thus, functional sensitivity analysis illustrates the fact that tunneling is determined by a large portion of the barrier, extending at least to the classical turning points.

Some practical problems associated with the functional sensitivity analysis strategy are worth mentioning. First, it is only first order. This is a severe problem. 
Indeed, the case where sensitivity is most interesting is for resonant processes. That is just the situation where the scattering wavefunction has large amplitude in the interaction region. However, at a resonance, perturbation theory (i.e. the Born expansion) breaks down because the Green's function is nearly singular. As such, first order results may not have much meaning in this case. Another problem pertains to the amount of information contained in the SC. It is a function of everything: total energy, initial and final quantum states and arrangements, and molecular coordinates. It may be difficult to digest all this information when functional sensitivity analysis is applied to complex (i.e. noncollinear) reactions.

We do not pursue functional sensitivity analysis further. Instead, we proceed with the results of the brute force comparison.

\subsection{The Potentials: LSTH vs. DMBE}

We have performed a brute force comparison of the energy dependent cross sections for reaction (2.1) calculated from the LSTH and DMBE potentials. These are different functional representations of the $a b$ initio PES calculated by Liu and Siegbahn in Ref. 16, although the DMBE PES is fitted to more noncollinear points than in Ref. 16. The potentials differ most significantly in two general regions - in the noncollinear saddle point regions and in the long range van der Waals complex regions. Since the energy region of interest for the comparison in Fig. 2.1 is quite high, long range van der Waals attractions should not be important. Thus the relevant difference is in the bending potential of the $\mathrm{H}_{3}$ transition state. Figure 2.2 shows a contour plot of the LSTH PES for $180^{\circ}$ fixed angle geometries, including contours of the difference potential $\left(V_{L S T H}-V_{D M B E}\right)$ for those geometries near the saddle point. Here the LSTH barrier height is $0.15 \mathrm{kcal} / \mathrm{mol}$ greater than that of the DMBE PES. Figure 2.3 is the same as Fig. 2.2 except that the internuclear angle is fixed at $90^{\circ}$. In this case the difference at the saddle point is up to $0.51 \mathrm{kcal} / \mathrm{mol}$, a reasonably significant quantity. Table 2.1 summarizes the saddle point differences for four fixed angle cuts of the surfaces. This shows that the greatest absolute difference is for $90^{\circ}$ geometries, and the greatest difference relative to the LSTH PES is for $120^{\circ}$ 


\begin{tabular}{|lllc||}
\hline \hline$\underline{\theta}$ & $\frac{V_{L}^{S P}}{30.0}$ & $\frac{\Delta V_{L-D}^{S P}}{0.51}$ & $\frac{\Delta V_{L-D}^{S P} / V_{L}^{S P}(\%)}{1.7 \%}$ \\
$90^{\circ}$ & 16.1 & 0.36 & $2.2 \%$ \\
$120^{\circ}$ & 11.5 & 0.16 & $1.4 \%$ \\
$150^{\circ}$ & 9.8 & 0.15 & $1.3 \%$ \\
$180^{\circ}$ & 2.8 \\
\hline
\end{tabular}

Table 2.1: Summary of relevant differences between the LSTH and DMBE potentials. The units of energy are $\mathrm{kcal} / \mathrm{mol}$. The angle $\theta$ is in valence coordinates, and as such is the angle included by the three $\mathrm{H}$ atoms. The relative difference between the two potentials is $c a$. uniform, but the absolute difference is more than a factor of 3 greater in the $\mathrm{L}$-shaped configuration $\left(90^{\circ}\right)$ than in the collinear configuration $\left(180^{\circ}\right)$. Thus, the LSTH PES has a tighter bend potential in the transition state region than does the DMBE PES.

geometries with the LSTH potential being larger in all cases. The percent difference is between $1.3 \%$ and $2.2 \%$ for all fixed angle surfaces considered.

\subsection{The Dynamics: Results and Discussion}

The transition state bending potential primarily determines the extent of rotational transitions. Therefore, we wish to analyze the sensitivity of the rotational product state distribution to these small changes in the bending PES. To do this we have calculated partial cross sections, i.e. cross sections for fixed total angular momentum $J$ given by

$$
\sigma_{v^{\prime}, j^{\prime} \mid v, j}(E, J)=\frac{\pi}{k_{v, j}^{2}} \times \frac{2 J+1}{2 j+1} \sum_{l^{\prime}, l}\left|S_{v^{\prime}, j^{\prime}, l^{\prime}-v, j, l}(E, J)\right|^{2},
$$

for $J=0,1$ and 10 . We considered $J=10$ because it is one of the largest terms in the sum over $J$. We considered low $J$ partial cross sections because the least energy reaction path is on the collinear surface. The transition state and collision complexes are collinear species. Since low values of $J$ roughly correspond to low impact parameters, i.e. head on collisions, these partial cross sections will be most important for a sensitivity study of scattering resonances presumably caused by collinear species. 


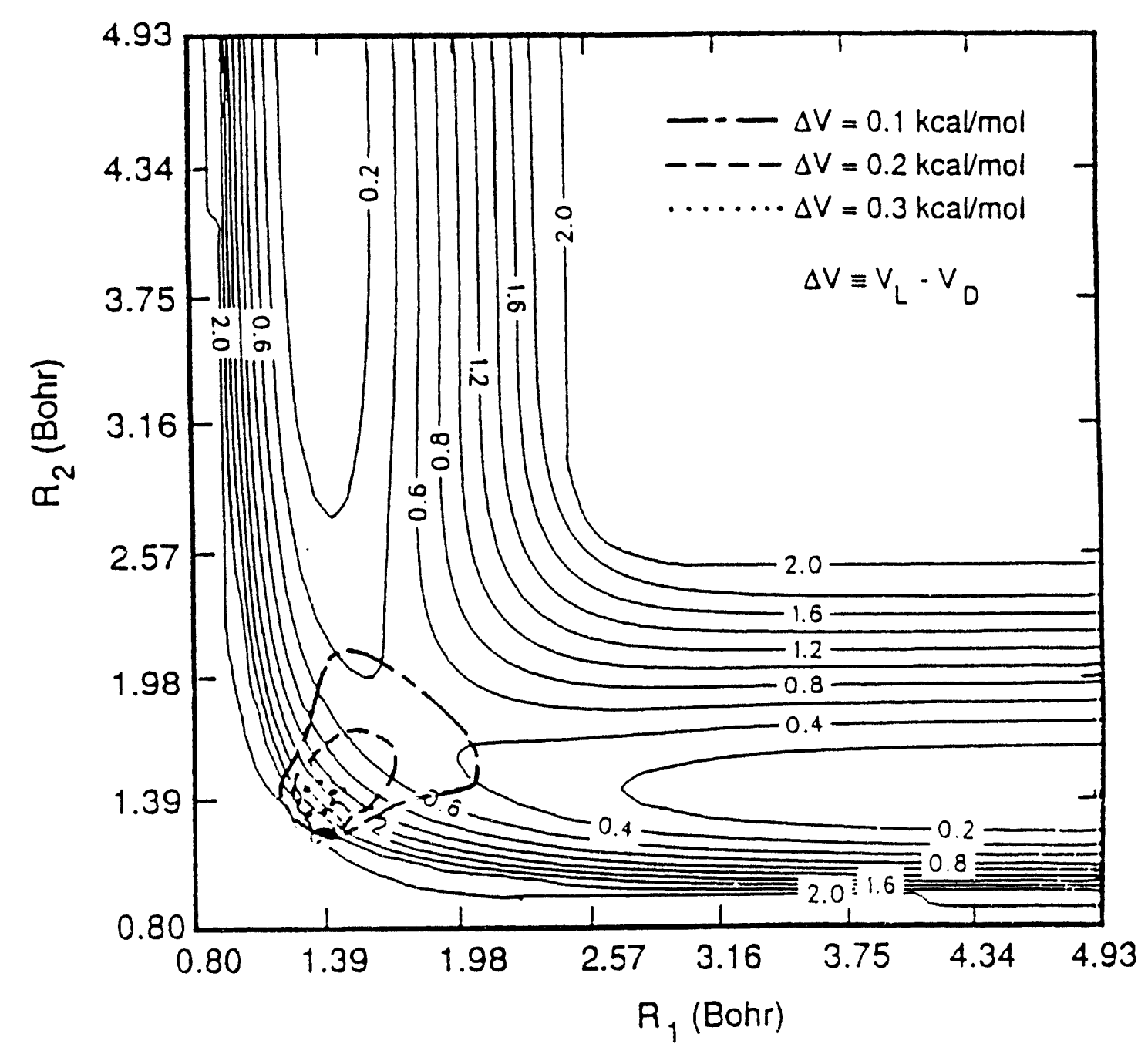

Figure 2.2: Contour plot of the LSTH potential energy surface at fixed internuclear angle $180^{\circ}$, cutoff at $2.0 \mathrm{eV}$. Also shown are the contours of the difference potential $\left(V_{L S T H}-V_{D M B E}\right)$ at $180^{\circ}$ where dotdash $=0.1$, dash $=0.2$, and dot $=0.3 \mathrm{kcal} / \mathrm{mol}$. The two surfaces differ by $0.15 \mathrm{kcal} / \mathrm{mol}$ (LSTH is higher) at the classical barrier geometry. 


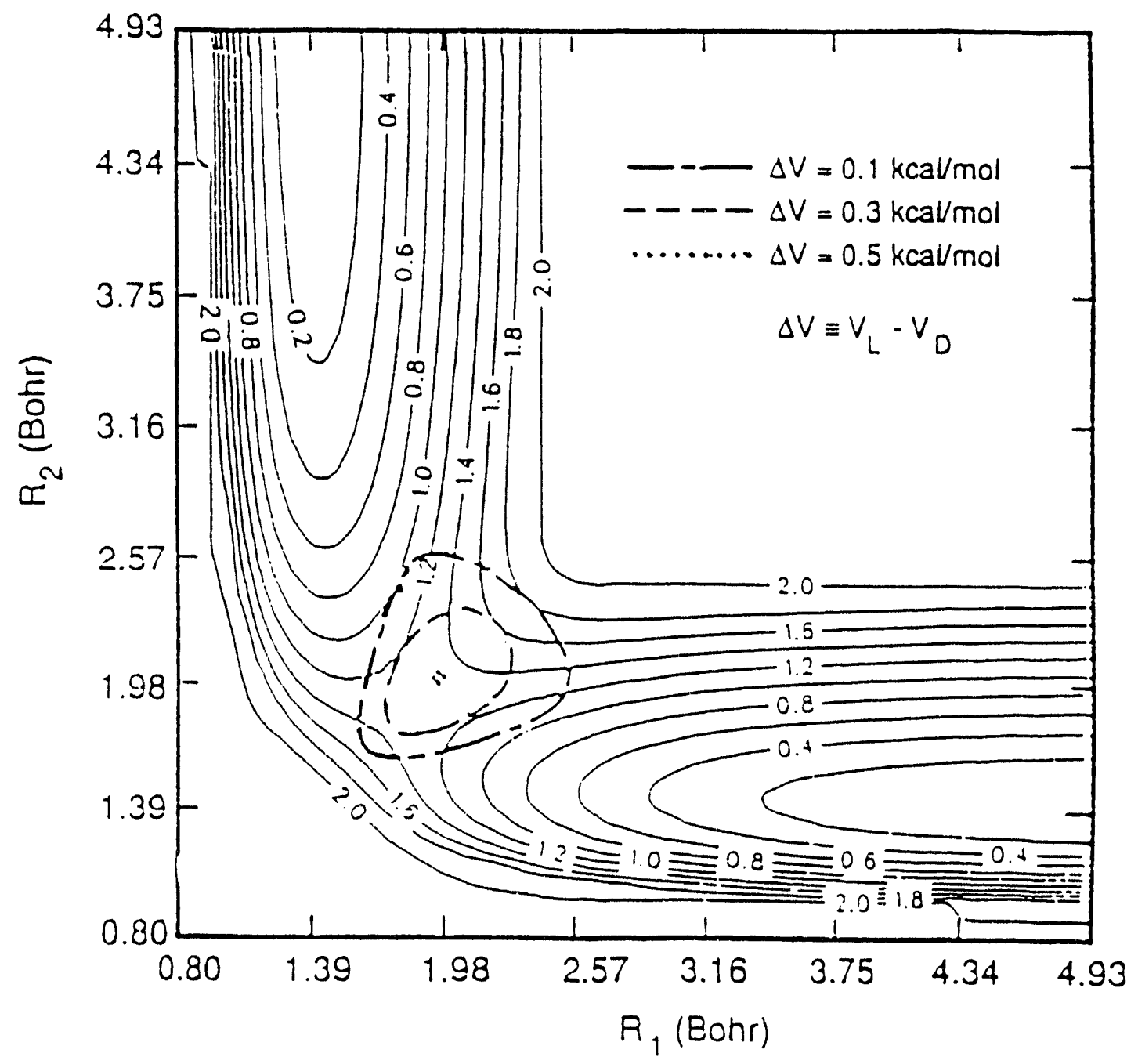

Figure 2.3: Contour plot of the LSTH potential energy surface at fixed internuclear angle $90^{\circ}$, cutoff at $2.0 \mathrm{eV}$. Also shown are the contours of the difference potential $\left(V_{L S T H}-V_{D M B E}\right)$ at $90^{\circ}$ where dotdash $=0.1$, dash $=0.3$, and dot $=0.5 \mathrm{kcal} / \mathrm{mol}$. The two surfaces differ by $0.51 \mathrm{kcal} / \mathrm{mol}$ (LSTH is higher) at this fixed angle saddle point geometry. 
We present the results for $J=0$ and 10 below $(J=0$ and 1 results are essentially identical except for the $2 J+1$ degeneracy).

Figure 2.4 shows the energy dependence of the partial cross sections $(J=0)$ for the transitions $(v=0, j=0)$ to $\left(v^{\prime}=1, j^{\prime}=1,3\right)$ resulting from the two potentials over the total energy range $0.8-1.5 \mathrm{eV}$. First, note the sharp resonance in the $(v=0, j=0)$ to $\left(v^{\prime}=1, j^{\prime}=1\right)$ partial cross section at $1.0 \mathrm{eV}$. In general, partial cross sections show resonance structure while the full cross section does not [25]. That is, as $J$ increases, features in the partial cross section move to higher energy and also broaden out (see below), so that the sum over partial waves (i.e. $J$ values) tends to produce a cross section with less structure than the individual partial cross sections.

The second important result seen in Fig. 2.4 is that for each transition, the partial cross sections for $J=0$ are qualitatively, and for most energies quantitatively identical for the LSTH and DMBE potentials. Figure 2.4, then, indicates very weak sensitivity to the difference in the bending PES for these transitions. Figure 2.5 shows the final vibrational and rotational product state distribution for $J=0$ at 1.2 $e V$, the energy of the largest experimentally observed resonance. Here we also see insensitivity to the difference PES being considered. We conclude that low $J$ results are insensitive to these specific small changes in the bending potential.

Figures 2.6 and 2.7 show the same quantities as Figs. 2.4 and 2.5, except for $J=10$, over the energy range $0.9-1.3 \mathrm{eV}$. Focusing on the $(v=0, j=0)$ to $\left(v^{\prime}=1, j^{\prime}=1\right)$ transition in Fig. 2.6, one sees that the resonance has been broadened by about a factor of 3 , and shifted up to $1.2 \mathrm{eV}$. Also, although one now detects a somewhat greater degree of sensitivity to the difference PES, the energy dependencies of the two cross sections have the same topology. The DMBE cross section is higher than the LSTH cross section because the two potentials differ most in the barrier heights, with DMBE having a smaller barrier height. Other than that, Figs. 2.6 and 2.7 show no qualitatively significant sensitivity, from which we conclude that high $J$ results are fairly insensitive to the difference between the two potentials. 


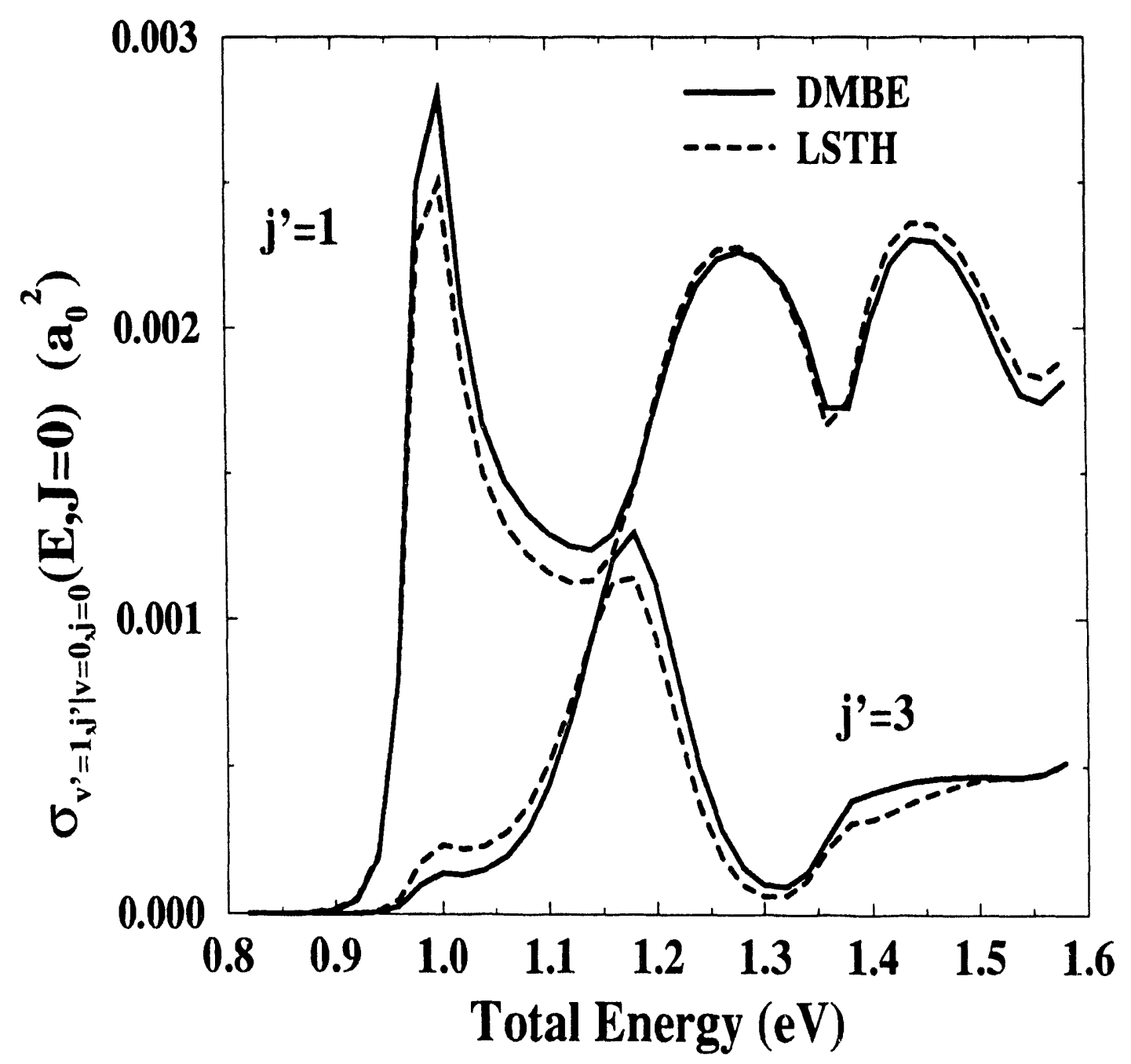

Figure 2.4: Partial cross sections for $\mathrm{H}+\mathrm{H}_{2}$ transitions $(v=0, j=0)$ to $\left(v^{\prime}=1, j^{\prime}=\right.$ $1,3)$, resulting from LSTH and DMBE PESs. The $\left(v^{\prime}=1, j^{\prime}=1\right)$ curve demonstrates a resonance at $E=1.0 \mathrm{eV}$, which is washed out in the partial wave expansion. These partial cross sections are insensitive to the difference between the LSTH and DMBE PESs, although there are quantitative differences. 


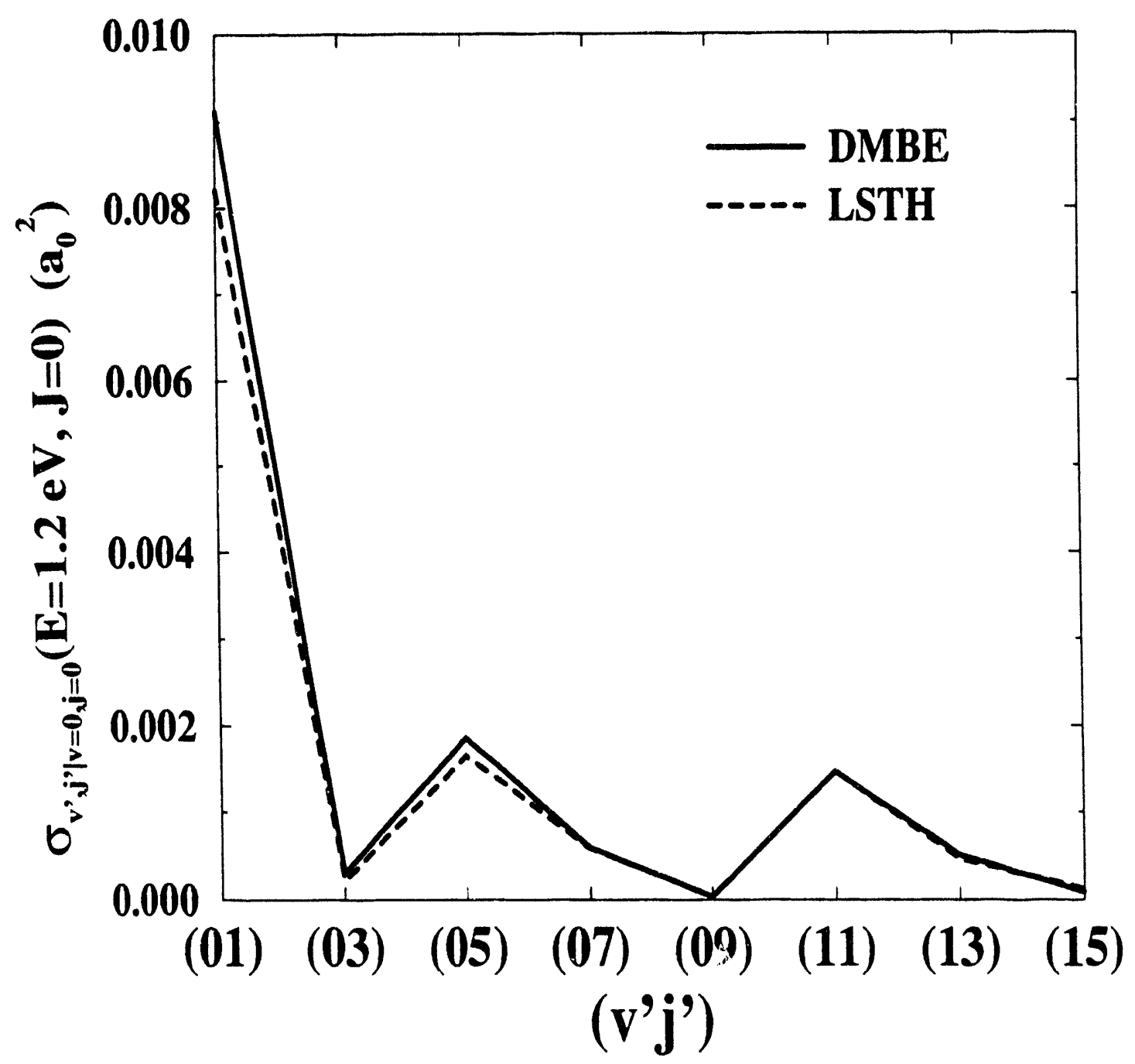

Figure 2.5: Product state distributions for $\mathrm{H}+\mathrm{H}_{2}(v=0, j=0)$ resulting from LSTH and DMBE with $J=0, E=1.2 \mathrm{eV}$. This product state distribution is quantitatively insensitive to the difference between the LSTH and DMBE PESs. 


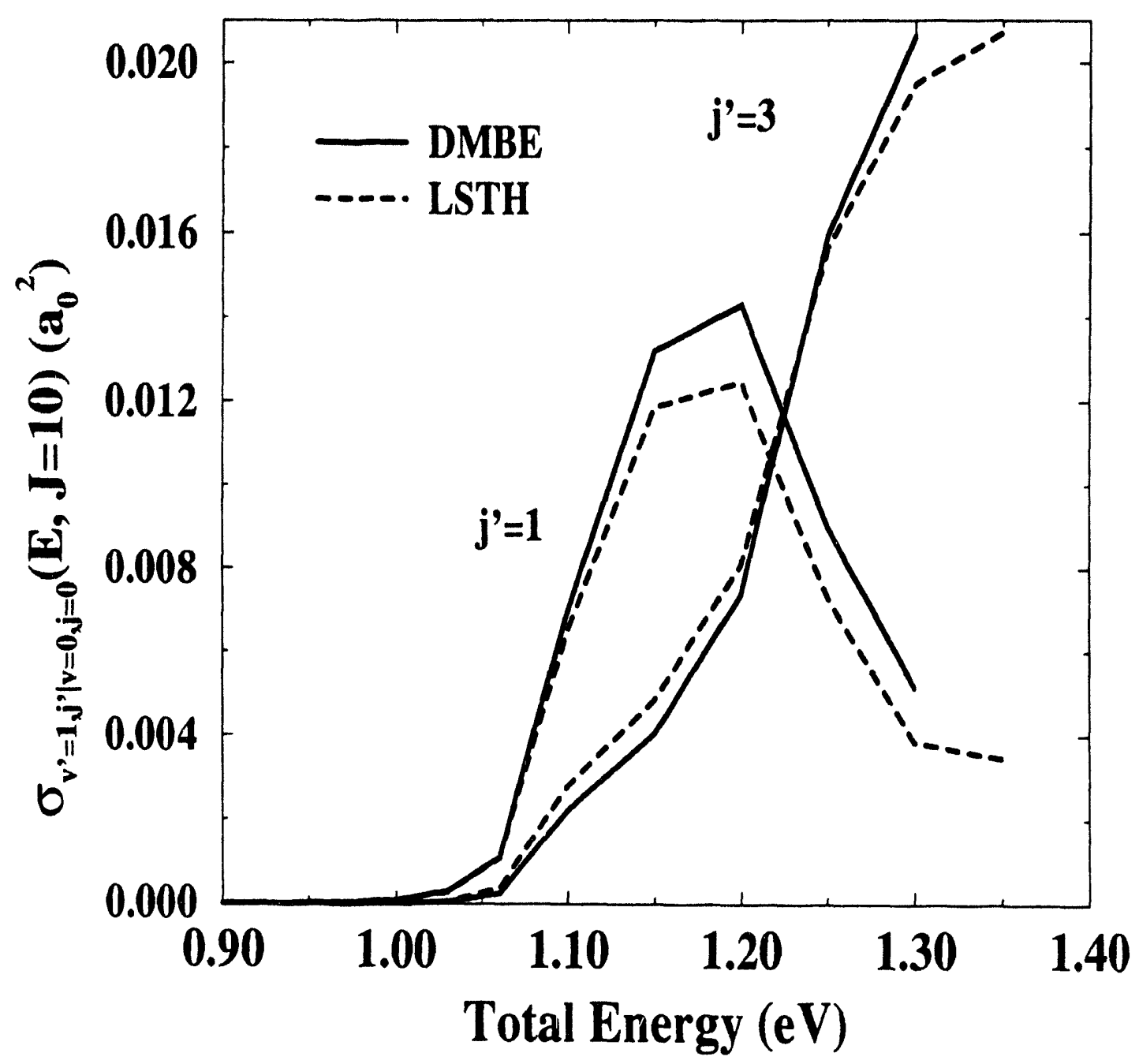

Figure 2.6: Partial cross sections for $\mathrm{H}+\mathrm{H}_{2}$ transitions $(v=0, j=0)$ to $\left(v^{\prime}=\right.$ $\left.1, j^{\prime}=1,3\right)$. The resonance is broadened by a factor of $c a .3$, and is shifted to $E=$ $1.2 \mathrm{eV}$. This $J$-dependent resonance energy shift is responsible for washing out the sharp resonant feature when performing the partial wave expansion. The partial cross sections are slightly more sensitive to the difference between the LSTH and DMBE PESs for $J=10$ than for the $J=0$ case. The energy dependence predicted by LSTH is qualitatively identical to that from DMBE. 


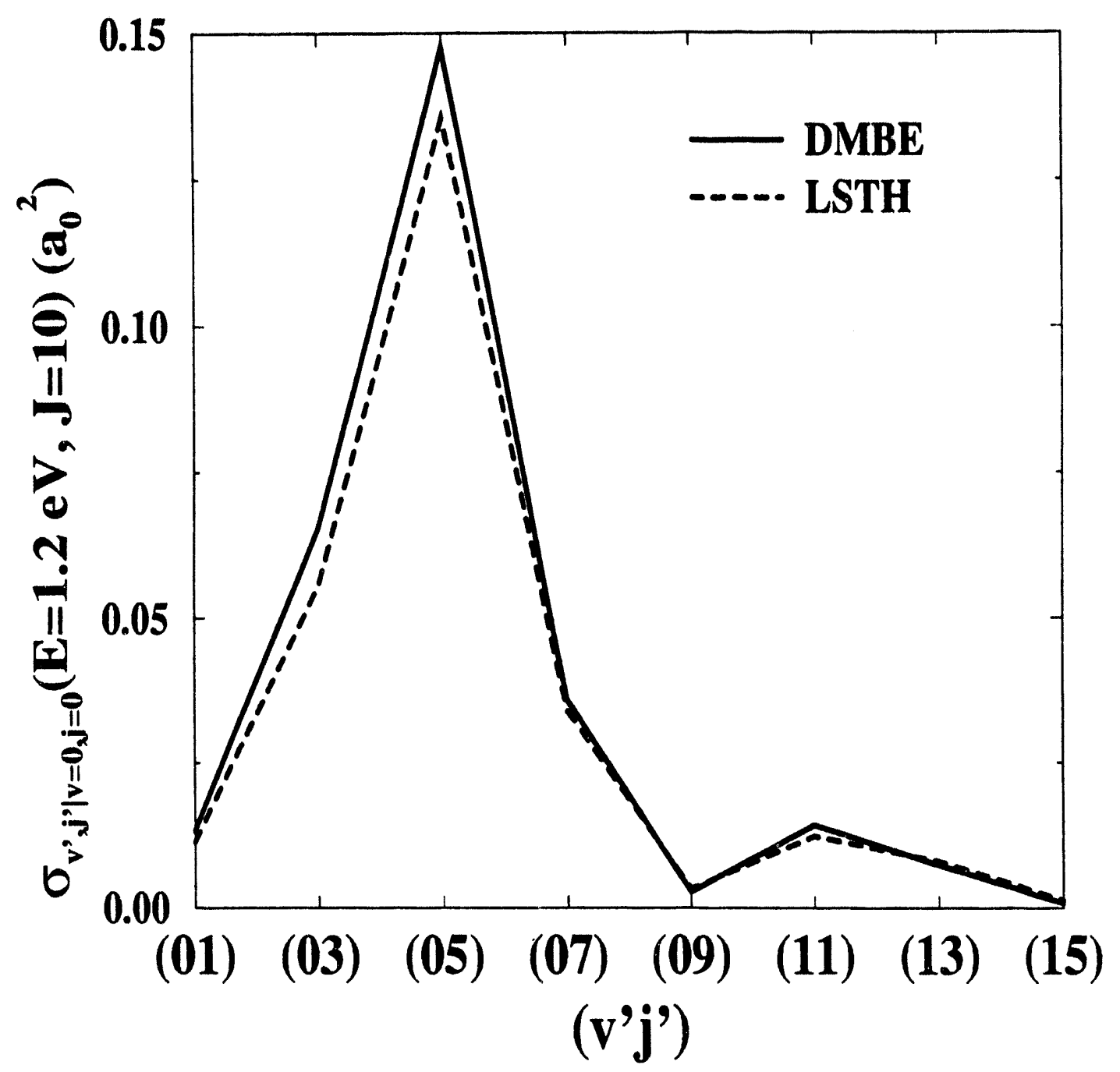

Figure 2.7: Product state distributions for $\mathrm{H}+\mathrm{H}_{2}(v=0, j=0)$ resulting from LSTH and DMBE with $J=10, E=1.2 \mathrm{eV}$. This product state distribution is quantitatively insensitive to the difference between the LSTH and DMBE PESs. The product distribution peaks at $j^{\prime}=5$ for this case of non-zero total angular momentum. 


\subsection{Concluding Remarks}

We outlined the derivation of the $S$-matrix version of the Kohn variational principle, and demonstrated how to apply functional sensitivity analysis within this computational framework. In addition, we discussed the complementarity of brute force and functional sensitivity analysis. We applied brute force sensitivity analysis to the $\mathrm{H}+\mathrm{H}_{2}$ reaction, comparing partial cross sections resulting from the LSTH and DMBE PESs. We found that the theoretical cross sections shown in Fig. 2.1 do not change significantly when the LSTH potential is replaced by the DMBE. The computed cross sections are thus fairly insensitive to changes in the potential energy surface. We conclude that the theoretical cross sections are accurate.

After these results were published [28], an experiment was performed by Kliner et al. [34] which measured the energy dependence of relative cross sections for reaction $(2.1)$. When these relative cross sections are normalized to the theoretical cross sections in Ref. 13, the energy dependence shown in shown in Fig. 2.8 results. The abscissa in Fig. 2.8 is translational energy, corresponding to total energies in the range $1.15-1.28 \mathrm{eV}$. This is the energy range for which Nieh and Valentini $[10,11]$ observe the sharpest resonance structure, as seen in Fig. 2.1. The results of Kliner et al. show no resonance structure, and agree quantitatively with theory over the entire energy range. Although this is not a proof that the Kliner et al. measurement is more reliable than that of Nieh and Valentini, the agreement shown in Fig. 2.8 is truly impressive. Indeed, the detection scheme employed by Kliner et al. is $(2+1)$ resonance enhanced multiphoton ionization (REMPI) followed by time of flight mass spectrometry. On the other hand, the detector used by Nieh and Valentini is based on coherent anti-Stokes Raman scattering spectroscopy (CARS), which requires more and stronger lasers than in REMPI. The implication here is that the product state distribution observed by Nieh and Valentini may not be nascent, but rather, the result of interference with a strong laser field [35]. A third independent measurement of this product state distribution may be required to establish the results of Kliner et al. as definitive.

The results shown in Fig. 2.8 are both disappointing and exciting. It is 


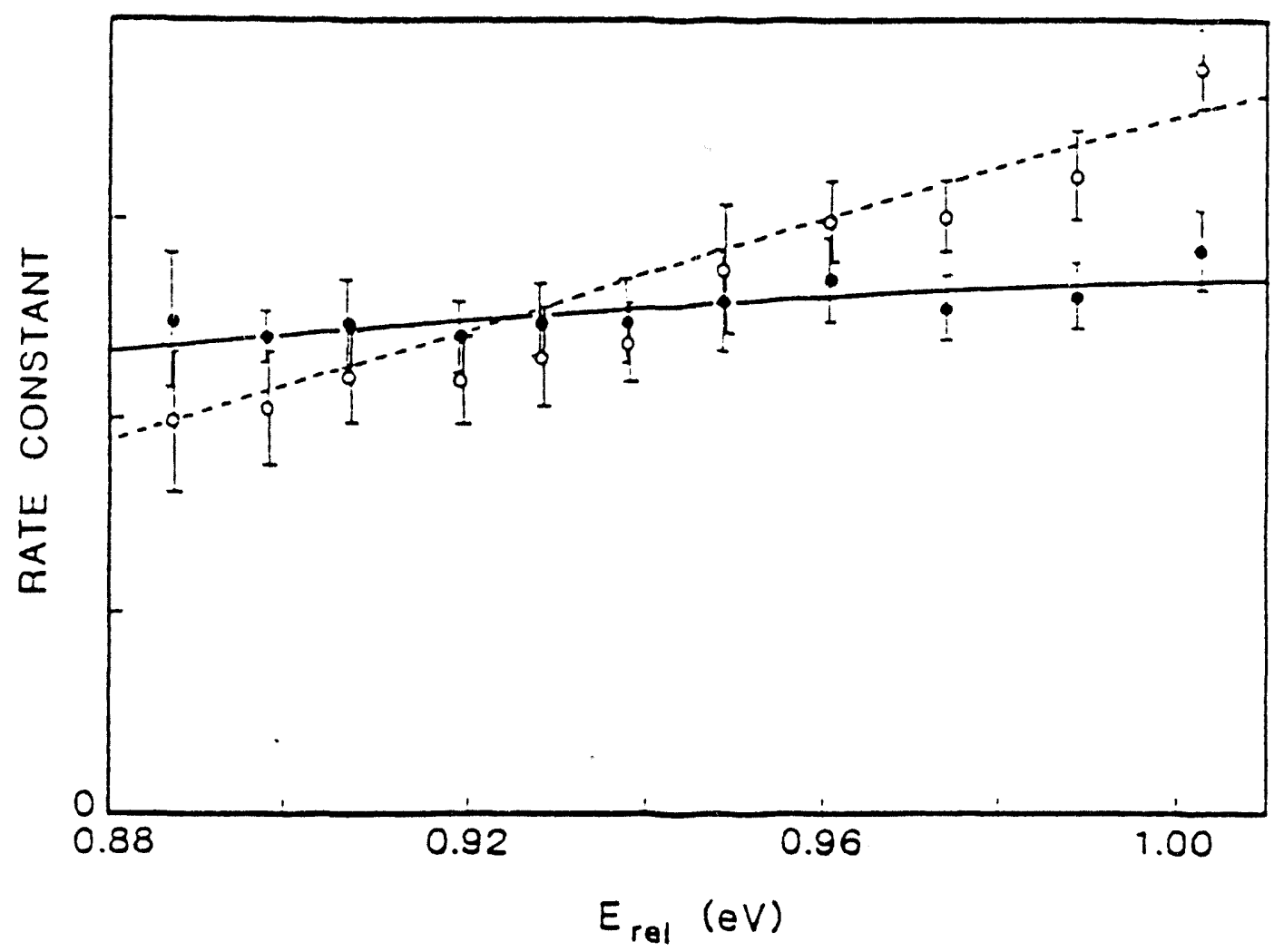

Figure 2.8: Experimental (points) and theoretical (curves) cross sections for the $\mathrm{H}+\mathrm{H}_{2}$ transitions $(v=0, j=0)$ to $\left(v^{\prime}=1, j^{\prime}=1,3\right)$, where the final state $j^{\prime}=1$ is the solid points and the solid curve, whereas $j^{\prime}=3$ is the open points and the dashed curve. The abscissa is translational energy relative to the $\mathrm{H}_{2}(v=0, j=0)$ energy level. These translational energies span a total energy range $E=1.15-1.28 \mathrm{eV}$, where Vieh and Valentini observed resonance structures. The ordinate is the rate constant for a very sharp energy distribution, which is a velocity times a cross section. The experiment observes smooth energy dependence, in quantitative agreement with the (appropriately averaged) theoretical results. This agreement ushers in the era of $a b$ initio reaction dynamics. 
intriguing that a collision complex of neutral $\mathrm{H}_{3}$ in its ground electronic state might be observable. That the truth seems to be contrary is disappointing $[36,37]$. On the other hand, the level of detail and agreement in Fig. 2.8 is unprecedented. The agreement indicates that the cross sections for this reaction are not extraordinarily sensitive to errors in the PES. If there were such extreme sensitivity, considering the accuracy of the LSTH PES, quantum reactive scattering calculations would be completely unreliable! That reactive scattering calculations are reliable ushers in the exciting era of $a b$ initio reaction dynamics.

The computational effort required by the scattering calculations shown here is not small. When attempting to treat more complex systems, i.e. systems with higher translational energies, heavier masses, greater exoergicities, stable intermediates, or more atoms, the added computational expense in both time and memory may make the calculation intractable. To a large extent, that is becasue the present methodology requires explicit construction of the outgoing wave Green's function. The remainder of this dissertation is devoted to developing alternative techniques in quantum reactive scattering theory which can be applied to more complex reactive systems.

\section{References}

[1] F. Webster and J. C. Light, J. Chem. Phys. 85, 4744 (1986).

[2] K. Haug, D. W. Schwenke, Y. Shima, D. G. Truhlar, Y. Zhang, J. Z. H. Zhang, and D. J. Khouri, J. Phys. Chem. 90, 6757 (1986).

[3] A. Kuppermann and P. G. Hipes, J. Chem. Phys. 84, 5962 (1986).

[4] R. T. Pack and G. A. Parker, J. Chem. Phys. 87, 3888 (1987).

[5] J. Z. H. Zhang, Shih-I. Chu, and W. H. Miller, J. Chem. Phys. 88, 6233 (1988).

[6] G. C. Schatz, Chem. Phys. Lett. 150, 92 (1988). 
[7] D. P. Gerrity and J. J. Valentini, J. Chem. Phys. 79, 5202 (1983); 81, 1298 (1984).

[8] C. T. Rettner, E. E. Marinero, and R. N. Zare in Physics of Electronic and Atomic Collisions, Invited Papers from the XVIIIth ICPEAC, Berlin, July 27 - August 2, 1983, eds. J. Eichler, I. V. Hertel, N. Stolterfoht (North Holland, Amsterdam, 1984), p. 51.

[9] E. E. Marinero, C. T. Rettner, and R. N. Zare, J. Chem. Phys. 80, 4141 (1984).

[10] J. C. Nieh and J. J. Valentini, Phys. Rev. Lett. 60, 519 (1988).

[11] J. C. Nieh and J. J. Valentini, J. Chem. Phys. 92, 1083 (1990).

[12] R. E. Continetti, B. A. Balko, and Y. T. Lee, J. Chem. Phys. 93, 5719 (1990).

[13] J. Z. H. Zhang and W. H. Miller, Chem. Phys. Lett. 153, 465 (1988).

[14] D. E. Manolopoulos and R. E. Wyatt, Chem. Phys. Lett. 159, 123 (1989).

[15] J. M. Launay and M. Le Douı teuf, Chem. Phys. Lett. 163, 178 (1989).

[16] P. Siegbahn and B. Liu, J. Chem. Phys. 68, 2457 (1978).

[17] D. Truhlar and C. Horowitz, J. Chem. Phys. 68, 2466 (1978); 71, 1514(E) (1979).

[18] H. Rabitz, Chem. Rev. 87, 101 (1987).

[19] J. N. L. Connor, W. Jakubetz, and J. Manz, Mol. Phys. 35, 1301 (1978); 39, 799 (1980).

[20] D. E. Manolopoulos and R. E. Wyatt, J. Chem. Phys. 92, 810 (1990).

[21] J. D. Kress, Z. Bačić, G. A. Parker, and R. T. Pack, J. Phys. Chem. 94, 8055 (1990).

[22] S. Takada, A. Ohsaki, and H. Nakamura, J. Chem. Phys. 96, 339 (1992).

[23] S. Shi and H. Rabitz, J. Chem. Plys. 86, 6190 (1987). 
[24] A. J. C. Verandas, F. B. Brown, C. A. Mead, D. G. Truhlar, and N. C. Blais, J. Chem. Phys. 86, 6258 (1987).

[25] J. Z. H. Zhang and W. H. Miller, J. Chem. Phys. 91, 1528 (1989).

[26] The $S$-matrix version of the Kohn variational principle has been used earlier in the field of nuclear physics; see M. Kamimura, Progr. Theoret. Phys. Suppl. 62, 236 (1977).

It has subsequently been used also in the field of electron-molecule scattering; see C. W. McCurdy, T. N. Rescigno, and B. I. Schneider, Phys. Rev. A 36, 2061 (1987); Phys. Rev. Lett. 63, 248 (1989); C. W. McCurdy and T. N. Tescigno, Phys. Rev A 39, 4487 (1989).

[27] For a more detailed discussion of the $S$-matrix version of the Kohn variational principle, see L. F. X. Gaucher, Ph.D. thesis, U.C. Berkeley, 1993.

[28] A brief summary of these sensitivity analysis results appeared previously in the following article:

S. M. Auerbach, J. Z. H. Zhang, and W. H. Miller, J. Chem. Soc. Faraday Trans. 86, 1701 (1990).

[29] W. H. Press, W. T. Vetterling, S. A. Teukolsky, and B. P. Flannery, Numerical Recipes in Fortran: The Art of Scientific Computing - Second Edition (Cambridge University Press, New York, 1992) pg. 410.

[30] E. C. Kemble, The Fundamental Principles of Quantum Mechanics with Elementary Applications (Dover, New York, 1958), pp. 410-415.

[31] L. S. Rodberg and R. M. Thaler, Introduction to the Quantum Theory of Scattering (Academic, New York, 1967).

[32] W. H. Miller and B. M. D. D. Jansen op de Haar, J. Chem. Phys. 86, 6213 (1987).

[33] A. Messiah, Quantum Mechanics, Vol. I(John Wiley and Sons, New York, 1958) pp. 231-241. 
[34] D. A. V. Kliner, D. E. Adelman, and R. N. Zare, J. Chem. Phys. 94, 1069 (1991).

[35] M. Shapiro, private communication.

[36] For a discussion of the possibility of experimentally observing resonances in the $\mathrm{H}+\mathrm{H}_{2}$ reaction, see W. H. Miller and J. Z. H. Zhang, J. Phys. Chem. 95, 12 (1991).

[37] For a discussion of classically allowed collision complexes in the $\mathrm{D}+\mathrm{H}_{2}$ system, see F. J. Aoiz, V. J. Herrero, and V. Sáez Rábanos, J. Chem. Phys. 97, 7423 (1992). 


\section{Chapter 3}

\section{Power Series Green's Function}

\subsection{Introduction}

The computational effort required by an exact quantum calculation grows exponentially with the size of the system. Accordingly, the amount of information obtained from a quantum calculation grows exponentially as well. The most extreme case is encountered when one studies the dynamics of a pure state in which all the relative phase information is required. It therefore seems reasonable that the treatment of mixed states, which provides less detailed dynamical information, should be less computationally demanding and thus more applicable for the study of larger chemical systems. For example, the canonical rate constant for a bimolecular chemical reaction can be expressed as

$$
k(T)=\left[2 \pi \hbar Q_{r}(T)\right]^{-1} \int_{-\infty}^{\infty} d E e^{-\beta E} N(E)
$$

where $\beta=\left(k_{B} T\right)^{-1}, k_{B}$ is Boltzmann's constant, and $Q_{r}(T)$ is the reactant partition function including relative translational motion, per unit volume. In Eq. (3.1) $N(E)$ is the microcanonical cumulative reaction probability, which in turn is defined by [1]

$$
N(E)=\sum_{\mathbf{n}_{p}} \sum_{\mathbf{n}_{r}}\left|S_{\mathbf{n}_{p}, \mathbf{n}_{r}}(E)\right|^{2}
$$

where $\left\{S_{\mathbf{n}_{p}, \mathbf{n}_{r}}(E)\right\}$ is the $S$-matrix for total energy $E$. The sums in Eq. (3.2) are over all energetically allowed states of the reactants and products, denoted by quan- 
tum numbers $\mathbf{n}_{r}$ and $\mathbf{n}_{p}$, respectively. Since the $S$-matrix elements contain the most detailed dynamical information, they are most computationally demanding. A formally exact approach to obtain $k(T)$ or $N(E)$ which circumvents the need to carry out exact $S$-matrix calculations should in principle be more economical, since the information content in the former quantities is manifestly independent of system size. Two such formulations were given in terms of the analysis of reactive flux correlation functions by Yamamoto [2] and by Miller et al. [3, 4]. In the present Chapter we will use the latter formulation to calculate the cumulative reaction probability.

Considerable theoretical effort has been devoted to evaluating the thermal rate constant $k(T)$ by the flux correlation formalism of Miller et al. [3-18]. Less attention, however, has been given to the direct calculation of the cumulative reaction probability $N(E)$ [19-21]. The theory for directly computing $N(E)$ depends upon the microcanonical density operator $\delta(E-\hat{H})$, which is formally obtained from the outgoing wave energy Greens's function [22]. Recently, Seideman and Miller [23] showed how to use absorbing boundary conditions (ABC) to construct a convenient, well-behaved representation of the energy Green's function for use in $N(E)$ calculations. $A B C$ have been used in the past, primarily in wave packet propagations, for the study of laser-induced dissociation $[24,25]$ and reactive scattering $[26,27]$. In the context of a wavepacket propagation, absorbing boundary conditions facilitate the use of smaller spatial grids by eliminating spurious reflection from grid boundaries. In the present context, they are used to enforce outgoing wave boundary conditions in the Green's function, without explicitly constructing the outgoing waves (please see the next Chapter for a detailed discussion of $\mathrm{ABC}$ in reactive scattering). In fact, in their study of $\mathrm{H}+\mathrm{H}_{2}$ reaction probabilities, Seideman and Miller were able to compute $N(E)$ by focusing only on the strong chemical interaction region, making no explicit reference to the asymptotic reactant and product states that would be necessary in a full $S$-matrix calculation. As such, their work represents significant progress in the search for an efficient calculation of the microcanonical cumulative reaction probability.

The calculation of $N(E)$ by an absorbing boundary condition Green's function relies on the construction and inversion of a non-Hermitian Hamiltonian matrix. 
We first discuss efficient inversion algorithms. The most straightforward inversion technique is LU decomposition [28], which requires storage of the Hamiltonian matrix. Iterative methods [29], which don't necessarily require such storage, are an important alternative to $\mathbf{L U}$ decomposition for the solution of large systems. The Lanczos algorithm [30-32], in which a Hermitian matrix is reduced to tridiagonal form, can be used to diagonalize [31] or invert [32] a matrix with minimal storage. This technique has been used extensively to treat chemical systems with real Hamiltonians [33-43]. Whether the Lanczos algorithm is readily applicable to complex symmetric matrices is an open question [31, 39, 44-47]. The generalized minimum residual [48] method (GMRES), however, is an algorithm applicable for the solution of arbitrary linear systems. Although it has given impressive results $[49,50]$, when used with preconditioning [51], GMRES can be memory intensive (please see the next Chapter for a detailed discussion of iterative methods in linear system solving). With the intention of extending $N(E)$ calculations to larger systems, we have developed a new iterative method for matrix inversion which is especially suited for Hamiltonians with absorbing boundary conditions. The method is stable, requires very little memory (as opposed to GMRES), and can readily be preconditioned (as opposed to the SYMMLQ [32] algorithm). It is based on Fourier transforming the time-dependent wavepacket dynamics to obtain the energy-dependent reaction probabilities, and gives, in the present implementation, a power series energy Green's function [52].

Forming the matrix representation of the Hamiltonian operator and manipulating the Hamiltonian matrix to obtain the observable of interest can be computationally intensive. A discrete variable representation [53-55] (DVR) can ameliorate both of these difficulties. That is, the construction of the Hamiltonian matrix is particularly simple in a DVR because no multidimensional integrals involving the potential function are required. Also, the resulting matrix is sparse because the potential is diagonal, which expedites an iterative solution [37,38]. In the present research we use a sinc-function based DVR (vide infra) first developed by Colbert and Miller [56] for use in the $S$-matrix version of the Kohn variational principle $[57,58]$, and used subsequently for $S$-matrix calculations $[37,38]$ in addition to $N(E)$ calculations [23]. This is a uniform grid DVR which is constructed from an infinite set of points. It is 
then truncated [59] to the shape of the potential by deleting grid points where the wavefunction or Green's function is vanishingly small. The uniform distribution of grid points inherent in this DVR has demonstrated significant efficiency in treating the gross anharmonicity of potential functions in reactive scattering.

Thus, the present theory includes wavepacket propagation on a grid as a basic component. McCullough and Wyatt [60] performed the first such study over twenty years ago on the $\mathrm{H}+\mathrm{H}_{2}$ system. Since then Kosloff and co-workers [61] have incorporated many improvements to augment the efficiency of exact wavepacket propagations, such as the Fourier representation [62] of the kinetic energy operator. This is a uniform grid over a finite interval, as opposed to sinc-function DVR which involves a uniform grid over an infinite interval. It would be interesting to determine whether the efficiency of wavepacket propagation is equivalent based on these two uniform grid representations. That is, one might ask for which physical systems would one grid method be superior to the other. We will present a qualitative analysis, concluding that sinc-function DVR is preferable for the representation of a molecular reactive scattering system when a large number of grid points, necessary for the Fourier method, can be deleted from the sinc-function DVR basis.

We perform wavepacket evolution by propagating over many small time steps, where each short-time evolution is effected by matrix multiplication on a grid. For many years, matrix multiplication was deemed an inappropriate method for such propagation because of the highly oscillatory nature of the short-time coordinate propagator. This problem has been addressed by several workers [63-76] who incorporate, in one guise or another, a filter to damp the very high frequency components in the propagator that cause numerical problems and are usually unimportant to the dynamics. For example, Coalson [65] computed real time correlation functions via matrix multiplication by adding a small imaginary part to the time. Alternatively, Makri [69] deleted momenta greater than some $p_{\max }$ from the propagator to yield a well behaved effective kernel. This approach was shown to be useful for both matrix multiplication and Monte Carlo [77] evaluation of the time evolution operator. It is interesting to note that this latter approach will turn out to be mathematically identical to the sinc-function DVR of the short-time coordinate propagator. Inspired by the 
success of this effective propagator, we will present a generalization of Makri's effective kernel which might be more useful in a path integral [78] Monte Carlo evaluation of the real time propagator.

\subsection{General Methodology}

We begin the calculation of the cumulative reaction probability with an expression derived from the analysis of reactive flux correlation functions [4], given by

$$
N(E)=\frac{1}{2}(2 \pi \hbar)^{2} \operatorname{Tr}[\hat{F} \delta(E-\hat{H}) \hat{F} \delta(E-\hat{H})]
$$

where " $T r$ " denotes a quantum mechanical trace. The reactive flux operator $\hat{F}$ in Eq. (3.3) is most generally defined by

$$
\hat{F}=\frac{1}{i \hbar}[\hat{h}(f), \hat{H}]
$$

where $h$ is the step function

$$
h(\xi)= \begin{cases}1 & \xi>0 \\ 0 & \xi<0\end{cases}
$$

and $f(\mathbf{q})$ defines, via the equation $f(\mathbf{q})=0$, a dividing surface which separates reactants from products. Here q denotes all the internal molecular coordinates. The microcanonical density operator $\delta(E-\hat{H})$ is formally obtained from the outgoing wave energy Green's function via the relation [22]

$$
\delta(E-\hat{H})=-\frac{1}{\pi} \operatorname{Im} \hat{G}^{+}(E),
$$

where $\hat{G}^{+}(E)$ is defined by [79]

$$
\begin{aligned}
\hat{G}^{+}(E) & \equiv \lim _{\epsilon \rightarrow 0^{+}}(E+i \epsilon-\hat{H})^{-1} \\
& =\lim _{\epsilon \rightarrow 0^{+}} \frac{1}{i \hbar} \int_{0}^{\infty} d t e^{i(E+i \epsilon-\hat{H}) t / \hbar} .
\end{aligned}
$$

In Eqs. (3.7) and (3.8), adding an infinitesimal imaginary part to the energy $E$ provides the outgoing wave boundary conditions, and, in Eq. (3.8) can be viewed as providing a factor which ensures the convergence of the time integral [23]. 
Seideman and Miller recently showed [23], in the definition of $\hat{G}^{+}(E)$, that subtracting a coordinate dependent operator $i \epsilon(\mathbf{q})$ from the Hamiltonian $\hat{H}$ can be equivalent to adding a constant $i \epsilon$ to the energy $E$, so long as $\epsilon(\mathbf{q})$ is negligible in the chemical interaction region. This technique, analogous to the use of absorbing potentials in the field of time-dependent wavepacket propagation [24-27], leads to the definition of an absorbing boundary condition (ABC) Green's function. The following alternative, but formally exact expression results for $N(E)$ :

$$
N(E)=4 \operatorname{Tr}\left[\hat{G}_{A B C}^{*}(E) \hat{\epsilon}_{p} \hat{G}_{A B C}(E) \hat{\epsilon}_{r}\right]
$$

where

$$
\begin{aligned}
\hat{G}_{A B C}(E) & \equiv(E+i \hat{\epsilon}-\hat{H})^{-1} \\
& =\frac{1}{i \hbar} \int_{0}^{\infty} d t e^{i(E+i \hat{\epsilon}-\hat{H}) t / \hbar}
\end{aligned}
$$

and

$$
\begin{aligned}
& \hat{\epsilon}_{p}=\hat{\epsilon} \hat{h} \\
& \hat{\epsilon}_{r}=\hat{\epsilon}(1-\hat{h}) .
\end{aligned}
$$

Here $\hat{\epsilon}$ is a coordinate dependent operator, and $\hat{h}$ is the coordinate dependent step function operator which defines the reactive flux in Eq. (3.4). If Eq. (3.9) is evaluated in a discrete variable representation $[23,53-56,80]$ (DVR) in which case the absorbing potentials, $\hat{\epsilon}_{r}$ and $\hat{\epsilon}_{p}$, are diagonal, $N(E)$ becomes

$$
N(E)=4 \sum_{\mathbf{j}^{\prime} \mathbf{j}} \epsilon_{p}\left(\mathbf{q}_{\mathbf{j}^{\prime}}\right)\left|G_{A B C}\left(\mathbf{q}_{\mathbf{j}^{\prime}}, \mathbf{q}_{\mathbf{j}} ; E\right)\right|^{2} \epsilon_{r}\left(\mathbf{q}_{\mathbf{j}}\right),
$$

where $\left\{\mathrm{q}_{\mathrm{j}}\right\}$ are the grid points and $\mathbf{j}$ is a multidimensional grid point index. Equation (3.13) is the working formula used in the present Chapter. In what follows, we remove the "ABC" subscript from the $\mathrm{ABC}$ Green's function, with $\hat{G}(E)$ denoting the $\mathrm{ABC}$ Green's operator and $\mathrm{G}(E)$ the finite dimensional $A B C$ Green's matrix.

The computational challenge involved in Eq. (3.13) is clearly the evaluation of the matrix elements of $\mathrm{G}(E)$ which connect the reactant and product regions of 
configuration space. In previous applications [23], these were computed as the solution of the complex symmetric linear system

$$
(E+i \epsilon-\mathbf{H}) \mathbf{G}(E)=\mathbf{1}
$$

by LU decompsition [28]. We call this the direct method of solution. For most chemically realistic systems, direct solution would require the storage and manipulation of matrices larger than can be held in the central memory of modern computers. One of the most fruitful approaches, however, in the solution of large linear systems is the use of a grid representation for the Hamiltonian in conjunction with an iterative solution of the resulting sparse linear system [29]. This is because iterative methods do not require storage of the Hamiltonian, and are especially rapid when used to solve sparse systems.

We now describe a new iterative procedure to compute $\mathbf{G}(E)$ on a grid. The method is based on Eq. (3.11), that is, the integral representation of the ABC Green's function.

\subsection{Power Series Green's Function}

We construct a power series representation of the ABC Green's function by taking a finite upper limit $T$ for the time integral in Eq. (3.11), and by using $N$ evenly spaced quadrature points to evaluate the resulting integral. The former approximation is valid because the use of $\mathrm{ABC}$ to define the Green's function guarantees the convergence of the integral in finite time. The latter approximation generates the power series. Other representations of the ABC Green's function, which incorporate more sophisticated quadrature for the time integral, are possible and are discussed in the next Chapter. These modifications to Eq. (3.11) give the following power series Green's function (PSG)

$$
\hat{G}(E) \cong \frac{\Delta t}{i \hbar} \sum_{n=0}^{N} w_{n} \hat{M}^{n}
$$

where

$$
\hat{M}=e^{i(E+i \hat{\epsilon}-\hat{H}) \Delta t / h}=e^{i E \Delta t / h} \hat{K}(\Delta t) .
$$


In Eq. (3.15) $\Delta t=T / N$, and $\left\{w_{n}\right\}$ are quadrature weights, e.g. for the extended trapezoidal rule $w_{n}=1-\left(\delta_{n 0}+\delta_{n N}\right) / 2$. In Eq. (3.16), $\hat{K}(\Delta t)$ is the propagator with absorbing boundary conditions for the duration $\Delta t$. As is common to the study of wave packet propagation, we have reduced the problem to finding the most accurate and efficient representation of the propagator [61, 81-83]. We use a simple and flexible short-time propagator (STP) developed by Feit, Fleck and co-workers [84-86] (also called [83] the kinetic referenced split-operator propagator) given by

$$
\hat{K}(t)=\hat{K}_{S T P}(t)+O\left(t^{3}\right),
$$

where

$$
\hat{K}_{S T P}(t)=e^{-i(\hat{V}-i \hat{\epsilon}) t / 2 \hbar} e^{-i \hat{T} t / \hbar} e^{-i(\hat{V}-i \hat{\epsilon}) t / 2 \hbar}
$$

and $\hat{H}=\hat{T}+\hat{V}$. This has been used [87-90] extensively in previous time-dependent calculations, and is a second-order propagator because it incorporates second-order commutation error in the symmetrization. It has the flexibility of being able to treat a time-dependent Hamiltonian, e.g. in a mixed quantum-classical time-dependent self-consistent field [90-92] framework, by taking a time step small enough that the Hamiltonian is approximately constant. Some al cernatives to the STP to be considered for future study will be discussed in the final Section of this Chapter, and in the next Chapter. We note that implementing propagators which are valid for longer times is tantamount to preconditioning [51] the system, i.e. making the Hamiltonian matrix more diagonally dominant.

\subsection{The Basis Set}

We represent the STP in Eq. (3.18) with sinc-function based DVR (SDVR) for each degree of freedom, where the sinc function is given by $\operatorname{sinc}(x) \equiv \sin (x) / x$. It was first described by Colbert and Miller [56] for use in the $S$-matrix version of the Kohn variational principle, and used subsequently for $S$-matrix calculations $[37,38]$ in addition to $N(E)$ calculations [23]. 


\subsubsection{SDVR vs. FFT}

Before giving the relevant matrix elements, we compare SDVR to the closely related Fourier grid method of Kosloff and co-workers $[61,62]$ which has been used extensively in previous wavepacket calculations. Both involve uniform grids in configuration space able to represent momenta up to

$$
p_{\max }=\frac{\hbar \pi}{\Delta x}
$$

where $\Delta x$ is the uniform grid spacing. In addition, both representations require roughly $[94,95] N \log _{2} N$ multiplications per propagation step, where $N$ is the size of the grid. The two methods differ, however, in the actual manner of propagation. The Fourier method requires multidimensional fast Fourier transforms [28] (FFT) to facilitate propagation, whereas SDVR relies on sparse matrix multiplication. As such, the grid used in SDVR calculations can easily be tailored [59] to the shape of the potential energy surface (PES), whereas the Fourier method requires the use of rectangular or L-shaped [96] grids in order to perform the FFT. The implication here is that the rectangular grids required for the Fourier method may waste points in unimportant or unphysical regions of configuration space, where the wavefunction or Green's function is vanishingly small. We conclude, therefore, that for the study of multidimensional systems where the relevant region of configuration space is approximately rectangular, the Fourier grid can be more computationally efficient. This situation can obtain, for example, in the study of photodissociation to a single fragment arrangement, or in gas-surface scattering [96]. But, for the study of multidimensional systems where the relevant region of configuration space is not rectangular, e.g. a gas phase reactive scattering system, SDVR should be more efficient if enough grid points are deleted from the basis.

\subsubsection{SDVR of the Free Particle Propagator}

We now give the sinc-function based DVR of the power series Green's function. For simplicity, we restrict our attention to a one-dimensional system. The multidimensional generalization is straightforward, and will be given afterwards. Letting 
$\left|u_{j}\right\rangle$ denote the $j^{\text {th }}$ SDVR basis ket, the matrix PSG becomes

$$
\mathbf{G}(E) \cong \frac{\Delta t}{i \hbar} \sum_{n=0}^{N} w_{n} \mathbf{M}^{n}
$$

where

$$
\begin{aligned}
{[\mathbf{M}]_{j^{\prime} j} } & =e^{i E \Delta t / \hbar}\left[\mathbf{K}_{S T P}(\Delta t)\right]_{j^{\prime} j} \\
{\left[\mathbf{K}_{S T P}(t)\right]_{j^{\prime} j} } & =e^{-i\left(V_{j^{\prime}}-i \epsilon_{j^{\prime}}\right) t / 2 \hbar}\left\langle u_{j^{\prime}}\left|e^{-i \hat{T} t / \hbar}\right| u_{j}\right\rangle e^{-i\left(V_{j}-i \epsilon_{j}\right) t / 2 \hbar}
\end{aligned}
$$

and $\hat{T}=\hat{p}^{2} / 2 m$. Equation (3.22) obtains from the fact that the PES and the absorbing potential are coordinate dependent operators. In Eq. (3.22), $V_{i}$ and $\epsilon_{i}$ correspond to the PES and the absorbing potential evaluated at the $i^{\text {th }}$ grid point, respectively. The SDVR of the free particle propagator is given in the fashion outlined by Colbert and Miller [56] in which one first considers a finite particle-in-a-box DVR. With $(N-1)$ functions and grid points and a grid spacing of $\Delta x$, the free particle propagator becomes

$$
\left[e^{-i \mathbf{T} t / \hbar}\right]_{j^{\prime} j}^{f i n i t e D V R}=\frac{2}{N} \sum_{n=1}^{N-1} \sin \left(\pi n j^{\prime} / N\right) \exp \left(\frac{-i \hbar \pi^{2} n^{2} t}{2 m \Delta x^{2} N^{2}}\right) \sin (\pi n j / N) .
$$

To obtain the SDVR of the free particle propagator, one takes the infinite $N$ limit of Eq. (3.23) keeping $\Delta x$ fixed, giving [97]

$$
\begin{aligned}
\left\langle u_{j^{\prime}}\left|e^{-i \hat{T} t / \hbar}\right| u_{j}\right\rangle & =\lim _{N \rightarrow \infty}\left[e^{-i \mathbf{T} t / \hbar}\right]_{j^{\prime} j}^{f i n i t e D V R} \\
& =\int_{0}^{1} d y \cos \left[\pi y\left(j^{\prime}-j\right)\right] \exp \left(\frac{-i \hbar \pi^{2} y^{2} t}{2 m \Delta x^{2}}\right)
\end{aligned}
$$

With the variable transformation $p=\hbar \pi y / \Delta x$, the free particle propagator in Eq. (3.24) takes on the more familiar form

$$
\left\langle u_{j^{\prime}}\left|e^{-i \hat{T} t / \hbar}\right| u_{j}\right\rangle=\int_{-p_{\max }}^{+p_{\max }} \frac{\Delta x d p}{2 \pi \hbar} e^{i p x_{j^{\prime}} / \hbar} e^{-i p^{2} t / 2 m \hbar} e^{-i p x_{j} / \hbar},
$$

where $p_{\max }$ is given in Eq. (3.19) and $x_{i}=i \Delta x$ for $i=j^{\prime}, j$.

The matrix element in Eq. (3.25) is noteworthy in three respects. First, it is the product of an integration weight and a finite grid spacing representation of the kernel. This product arises because DVR includes integration weights in the 
transformations. As $p_{\max }$ goes to $\infty$ (and $\Delta x$ goes to zero), the summation implied by matrix multiplication goes over into an integral, and the matrix element becomes

$$
\lim _{p_{\max } \rightarrow \infty}\left\langle u_{j^{\prime}}\left|e^{-i \hat{T} t / \hbar}\right| u_{j}\right\rangle=d x\left\langle x_{j^{\prime}}\left|e^{-i \hat{T} t / \hbar}\right| x_{j}\right\rangle_{\text {exact }},
$$

recovering the exact kernel of the free particle propagator multiplied by the infinitesimal integration weight. Second, the SDVR of the free particle propagator is easily evaluated giving the exact kernel times a smoothing factor that results from the finite grid spacing. Eq. (3.25) becomes

$$
\left\langle u_{j^{\prime}}\left|e^{-i \hat{T} t / \hbar}\right| u_{j}\right\rangle=\Delta x\left\langle x_{j^{\prime}}\left|e^{-i \hat{T} t / \hbar}\right| x_{j}\right\rangle_{\text {exact }} \times f_{\text {smooth }}\left(x_{j^{\prime}}, x_{j}, t ; m, \Delta x\right)
$$

where

$$
\begin{aligned}
f_{\text {smooth }}\left(x_{j^{\prime}}, x_{j}, t ; m, \Delta x\right) & =\frac{1}{2}[\operatorname{erf}(A+B)+\operatorname{erf}(A-B)] \\
A & =\left(\frac{i t}{2 m \hbar}\right)^{1 / 2} \frac{\hbar \pi}{\Delta x} \\
B & =\left(\frac{i m}{2 \hbar t}\right)^{1 / 2}\left(x_{j^{\prime}}-x_{j}\right) .
\end{aligned}
$$

In Eq. (3.28), erf $(z)$ is the error function of a complex variable [98]. The third, and perhaps most intriguing aspect of Eqs. (3.25), (3.27), and (3.28) is that they have been derived before in a completely different context by Makri [69]. In particular, she was seeking a well behaved (i.e. less oscillatory) representation of the short-time kernel for use in real time path integral. Monte Carlo calculations [63-68, 70-76] The advantage gained from the matrix element in Eqs. (3.27) and (3.28) derives from the asymptotic behavior of the smoothing function $[69,81]$, namely

$$
\lim _{\hat{p} \rightarrow \infty}\left\langle u_{j^{\prime}}\left|e^{-i \hat{T} t / \hbar}\right| u_{j}\right\rangle=\operatorname{sinc}\left[\pi\left(x_{j^{\prime}}-x_{j}\right) / \Delta x\right]
$$

where

$$
\tilde{p} \equiv m\left(x_{j^{\prime}}-x_{j}\right) / t
$$

and in the above limit $p_{\max }$ (and, hence, $\Delta x$ ) is understood to be fixed. Thus, Makri's version of the propagator automatically includes the effect of high frequency phase 
cancellation that numerical quadrature and Monte Carlo algorithms have to work so hard to simulate. This cancellation is manifest in the damping of the free particle propagator matrix element for large $\tilde{p}$, i.e. for very high momentum paths. In fact, Makri found this propagator to be so well behaved, that wavepacket propagation by straightforward matrix multiplication was efficient and accurate. That is precisely the type of propagation being done in the present Chapter. It is analogous to the numerical matrix multiplication scheme of Berne and co-workers [99] used to compute the canonical density matrix by a discretized path integral in imaginary time. It is interesting to note that our numerical tests indicate that without the smoothing factor, the propagation becomes unstable and numerical overflow occurs. It is also worth noting that Kouri and co-workers [73-76] have developed a different approximation to the free particle coordinate propagator, similar in spirit to Makri's propagator, which neglects high momentum components. Thus, the SDVR of the STP gives the exact position representation of the STP, which, when placed on a grid with finite spacing, automatically filters out high frequency momentum components which are unimportant and difficult to integrate.

\subsection{Multidimensional Generalization}

For the multidimensional generalization of Eqs. (3.20)-(3.30), we consider the $F$-dimensional Hamiltonian operator given by

$$
\hat{H}=\sum_{\alpha=1}^{F} \hat{T}_{\alpha}+\hat{V}(\mathbf{q})
$$

where

$$
\hat{T}_{\alpha}=\frac{p_{\alpha}^{2}}{2 m_{\alpha}}
$$

and $\mathbf{q}=\left(q^{1}, \ldots, q^{F}\right)$ is an $F$-dimensional point in Cartesian space. We form an $F$-dimensional grid defined by

$$
q_{j_{\alpha}}^{\alpha}=j_{\alpha} \Delta q^{\alpha}, \quad \text { for } \quad j_{\alpha}=0, \pm 1, \pm 2, \ldots
$$


for each coordinate $q^{\alpha}$ and corresponding grid point index $j_{\alpha}$. The infinite grid is then truncated by adapting it to the shape of the PES. That is, the points $q_{j}$ are retained only if they satisfy the energy criterion [59] given by $V\left(\mathbf{q}_{\mathbf{j}}\right) \leq V_{\text {cut }}$, where $V_{\text {cut }}$ becomes a basis set convergence parameter. The corresponding ABC-DVR power series Green's function is still given by Eq. (3.20), where the matrix $M$ is now defined by

$$
\begin{aligned}
{[\mathbf{M}]_{\mathbf{j}^{\prime} \mathbf{j}} } & =e^{i E \Delta t / \hbar}\left[\mathbf{K}_{S T P}(\Delta t)\right]_{\mathbf{j}^{\prime} \mathbf{j}} \\
{\left[\mathbf{K}_{S T P}(t)\right]_{\mathbf{j}^{\prime} \mathbf{j}} } & =e^{-i\left(V_{\mathbf{j}^{\prime}}-i \epsilon_{\mathbf{j}^{\prime}}\right) t / 2 \hbar}\left[\prod_{\alpha=1}^{F}\left\langle u_{j_{\alpha}^{\prime}}^{\alpha}\left|e^{-i \hat{T}_{\alpha} t / \hbar}\right| u_{j_{\alpha}}^{\alpha}\right\rangle\right] e^{-i\left(V_{\mathbf{j}^{-}}-i \epsilon_{\mathbf{j}}\right) t / 2 \hbar}
\end{aligned}
$$

and $\mathbf{j}=\left(j_{1}, \ldots, j_{F}\right)$ is the $F$-dimensional grid point index alluded to in Eq. (3.13). In Eq. (3.35) $V_{\mathbf{j}}$ and $\epsilon_{\mathbf{j}}$ are the $F$-dimensional PES and absorbing potential, respectively; evaluated at $\mathrm{q}_{\mathbf{j}}$. Each of the factors in the direct product free particle matrix element in Eq. (3.35) is Eq. (3.27) evaluated with the appropriate mass and grid spacing, i.e.

$$
\left\langle u_{j_{\alpha}^{\prime}}^{\alpha}\left|e^{-i \hat{T}_{\alpha} t / \hbar}\right| u_{j_{\alpha}}^{\alpha}\right\rangle=\Delta q_{\alpha}\left\langle q_{j_{\alpha}^{\prime}}^{\alpha}\left|e^{-i \hat{T}_{\alpha} t / \hbar}\right| q_{j_{\alpha}}^{\alpha}\right\rangle_{\text {exact }} \times f_{s m o o t h}\left(q_{j_{\alpha}^{\prime}}^{\alpha}, q_{j_{\alpha}}^{\alpha}, t ; m_{\alpha}, \Delta q_{\alpha}\right) .
$$

In multidimensional SDVR the Hamiltonian matrix is sparse, which leads to $N(2+$ $F N^{1 / F}$ ) multiplications for each application of the matrix $\mathrm{M}$, where $N$ is the size of the grid $[37,38,56]$.

\subsection{Summary of the Methodology}

To conclude the description of the power series Green's function (PSG), we wish to underscore how the recursive calculation proceeds. To compute the $\mathrm{j}^{\text {th }}$ column of the ABC Green's function, denoted by $\mathbf{G}_{\mathbf{j}}$ with elements given by $\left(\mathbf{G}_{\mathbf{j}}\right)_{\mathbf{j}^{\prime}}=$ $G\left(\mathbf{q}_{\mathbf{j}^{\prime}}, \mathbf{q}_{\mathbf{j}}\right)$, one forms the dot product of the matrix in Eq. (3.20) with the $\mathbf{j}^{\text {th }}$ column of the identity matrix. As such, the $\mathrm{j}^{\text {th }}$ column of $\mathbf{G}(E)$ is

$$
\mathbf{G}_{\mathbf{j}} \cong \frac{\Delta t}{i \hbar} \sum_{n=0}^{N} w_{n} \mathbf{a}_{n \mathbf{j}}
$$

where the vectors $\left\{\mathbf{a}_{\mathbf{n} \mathbf{j}}\right\}$ are defined by

$$
\left(\mathbf{a}_{0 \mathbf{j}}\right)_{\mathbf{j}^{\prime}}=\left(\mathbf{I}_{\mathbf{j}}\right)_{\mathbf{j}^{\prime}}=\delta_{\mathbf{j}^{\prime} \mathbf{j}}=\prod_{\alpha=1}^{F} \delta_{j_{\alpha}^{\prime}, j_{\alpha}}
$$


and

$$
a_{n+1 j}=M a_{n j}
$$

with $M$ defined in Eq. (3.35). Equation (3.39) is the recursion relation which defines the iterative method.

We now present a numerical application of the PSG in the study of the cumulative reaction probability for the collinear $\mathrm{H}+\mathrm{H}_{2}$ reactive scattering system.

\subsection{Results and Discussion}

\subsubsection{The Coordinates}

We present the results of the calculation of the cumulative reaction probability for collinear $\mathrm{H}+\mathrm{H}_{2}$ over the total energy range of 0.37 to $1.27 \mathrm{eV}$, using the method described above. The availability of accurate PES's and dynamics calculations makes it a good benchmark system to use to study a new method. We use the Liu-Siegbahn-Truhlar-Horowitz $[100,101]$ (LSTH) PES for the calculations. The coordinates used for the calculations were the mass-weighted rectilinear normal modes $[56,102]$ referenced to the transition state on the LSTH PES. We denote the two dimensional coordinates by $\mathbf{q}=(x, y)$ where $x$ is the reaction coordinate and $y$ is the perpendicular vibrational coordinate, i.e. the anti-symmetric and symmetric stretch, respectively.

A primitive DVR grid is first laid down along the coordinates $q$. We choose the grid spacings in the manner suggested by Colbert and Miller [56]. That is, given a mass and energy scale for each degree of freedom, we fix the number of grid points per de Broglie wavelength, denoted by $n_{B}$. This gives the grid spacing for each degree of freedom as

$$
\Delta q_{\alpha}=\frac{2 \pi \hbar}{n_{B} \sqrt{2 m_{\alpha} E_{\alpha}}}
$$

For all studies at total energy $E$, we used $E_{\alpha}=E, m_{\alpha}=1060$ au (the mass-weighted system reduced mass), and $n_{B} \cong 4$ points per wave for both degrees of freedom. 
Next, the primitive grid is truncated to yield a non-rectangular grid. To avoid an overabundance of convergence parameters for later discussion we set the energy cutoff $V_{\text {cut }}=3.4 \mathrm{eV}$ for all calculations, which was found to give satisfactory convergence.

\subsubsection{The Absorbing Potential}

The absorbing potential in the ABC Green's function simulates the effect of outgoing wave boundary conditions. It does so by absorbing completely, without back reflection, any flux from the interaction region that encounters the edge of the grid in all energetically allowed arrangements. As previously discussed by many authors [23-27], the optimal form of the absorbing potential is one which absorbs probability as rapidly (in space) as possible, without reflection. We use one of the forms suggested by Seideman and Miller [23], a sigmoid function given by

$$
\begin{aligned}
\epsilon[z(\mathbf{q})] & =\frac{2 \lambda}{1+\exp \left[\left(z_{\max }-z\right) / \eta\right]} \\
z(\mathbf{q}) & =\max \left[R_{a}(\mathbf{q}), R_{c}(\mathbf{q})\right] \\
z_{\max } & =R_{\gamma}^{\max }, \quad \text { if } z(\mathbf{q})=R_{\gamma}(\mathbf{q}) \text { for } \gamma=a, c
\end{aligned}
$$

and $R_{a}\left(R_{c}\right)$ is the translational Jacobi coordinate in the reactant (product) arrangement. In the symmetric case of collinear $\mathrm{H}+\mathrm{H}_{2}$, we have $z_{\max }=R_{a}^{\max }=R_{c}^{\max }$, where we have used $z_{\max }=5$ au for most of the calculations. A typical grid is shown in Fig. 3.1 including the contours of the absorbing potential $\epsilon(\mathbf{q})$. The parameters $\lambda$ and $\eta$ are optimized by running appropriate convergence tests. For the convergence of the power series Green's function, we single out the parameter $\lambda$ as being especially important. This is clear from the following analysis. We let $\epsilon(\mathbf{q})=\lambda$ be a constant absorbing potential. If one takes a finite upper limit $T$ for the time integral [i.e. a finite value of $N$ in Eq. (3.20)], the error incurred is given by

$$
\left.\mathbf{G}(E)\right|_{f i n i t e T}=\mathbf{G}(E)\left[1-O\left(e^{-\lambda T / \hbar}\right)\right]
$$

Thus, we expect to see exponential convergence in the parameters $\lambda$ and $T$. Although this analysis is complicated by the use of a coordinate dependent absorbing potential, it seems plausible that to converge the PSG with as few iterations as possible, it may 
be beneficial to use larger values of $\lambda$ than in previous studies. Results of this analysis will be discussed.

\subsubsection{Reaction Probabilities}

Before stating the results, we wish to comment briefly on the scaling of the computational expense of the PSG with respect to central memory and CPU time. For an $F$-dimensional system with, on average, $n$ grid points per degree of freedom, the PSG scales as $[37,38,56] N_{R H S} N_{I T E R} F n N$ where $N_{R H S}$ is the number of reactant grid points for which a vector of $\mathrm{G}(E)$ is computed and $N_{I T E R}$ is the number of iterations per RHS. From our experience, this roughly scales as $F n N^{2}$. The PSG requires only three vectors of length $N$ for the essential recursion and summation, in addition to a very small number of vectors to enhance the speed of each iteration. Clearly this is where the PSG is favorable over direct methods.

The cumulative reaction probability for collinear $\mathrm{H}+\mathrm{H}_{2}$, computed by the method described above, is shown in Fig. 3.2. The circles represent the reaction probabilities computed by Bondi et al. $[103,104]$ using $R$-matrix propagation on the LSTH PES. The agreement is excellent over the whole energy range. In the energy range where there is only one energetically accessible asymptotic vibrational channel $[E \in(0.37,0.78 \mathrm{eV})]$, the error is always less than $1 \%$. In the higher energy regime, the error is always less then $1.6 \%$.

The energy dependence of $N(E)$ in Fig. 3.2 demonstrates the "staircase" structure given by the quantized vibrational levels of the activated complex. With only one such level accessible for lower energies, $N(E)$ rises quickly to unity. When the second level becomes energetically accessible, $N(E)$ makes another jump to $c a$. 1.6. The fact that $N(E)$ does not increase monotonically is the signature of transition state theory violating dynamics. The sharp (downward) peak at $E=0.87 \mathrm{eV}$ indir ates a resonance, i.e. a short lived collisior. complex of $\mathrm{H}_{3}$. This structure becomes washed out, however, in the full dimensional treatment (with zero total angular momentum) [23], because of the "averaging" over bending motions. 


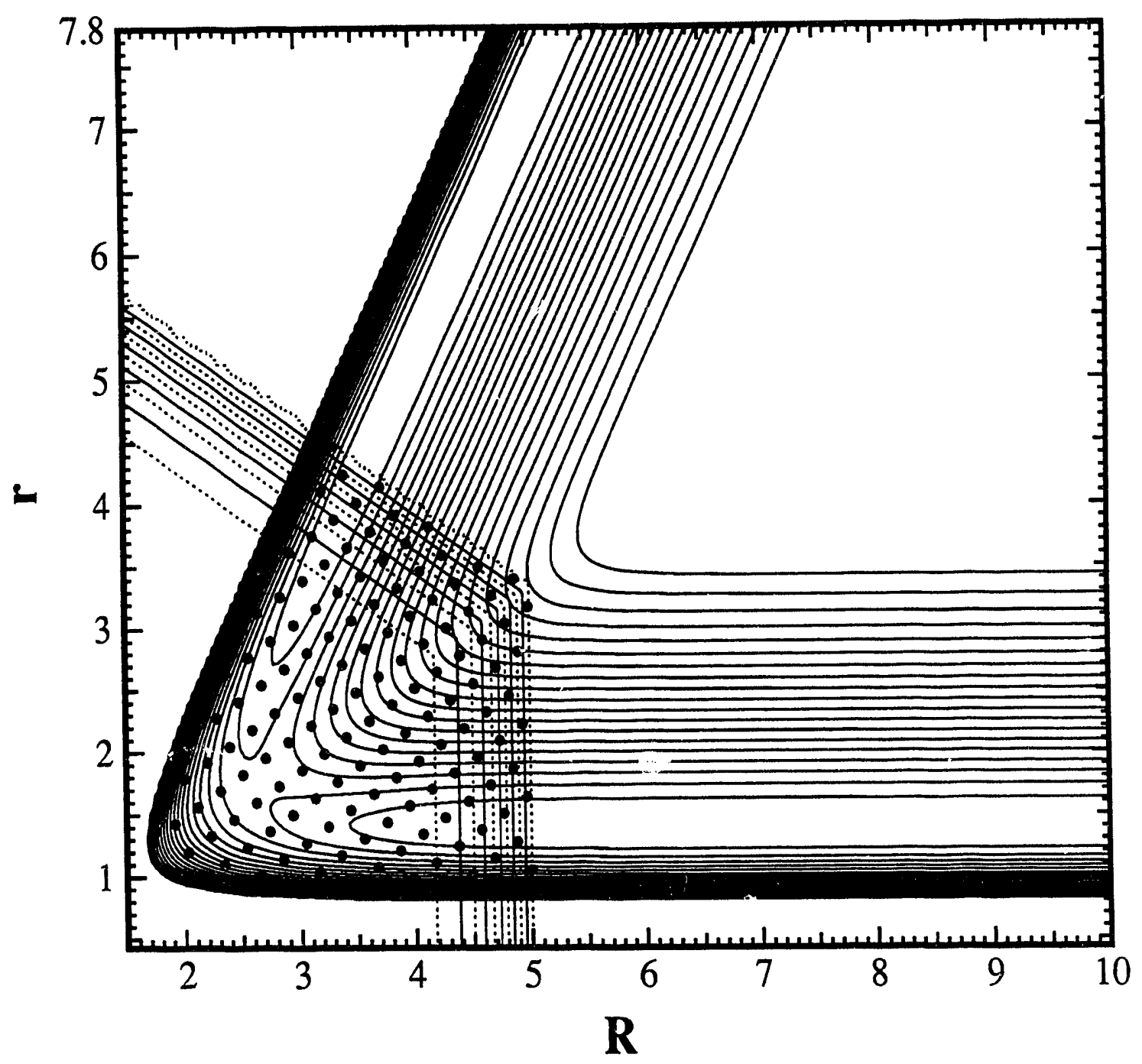

Figure 3.1: The configuration space required to converge the cumulative reaction probability. The contours are the LSTH potential surface as a function of the Jacobi coordinates $(R, r)$. The other contours show the sigmoid absorbing potential where $\lambda$ $=1.1 \mathrm{eV}$ and $\eta=0.27 \mathrm{au}$. The distribution of grid points shown was used for the convergence tests at $E=0.52 \mathrm{eV}$. This small region of configuration space is all that is required to obtain an averaged reaction probability such as $N(E)$. 


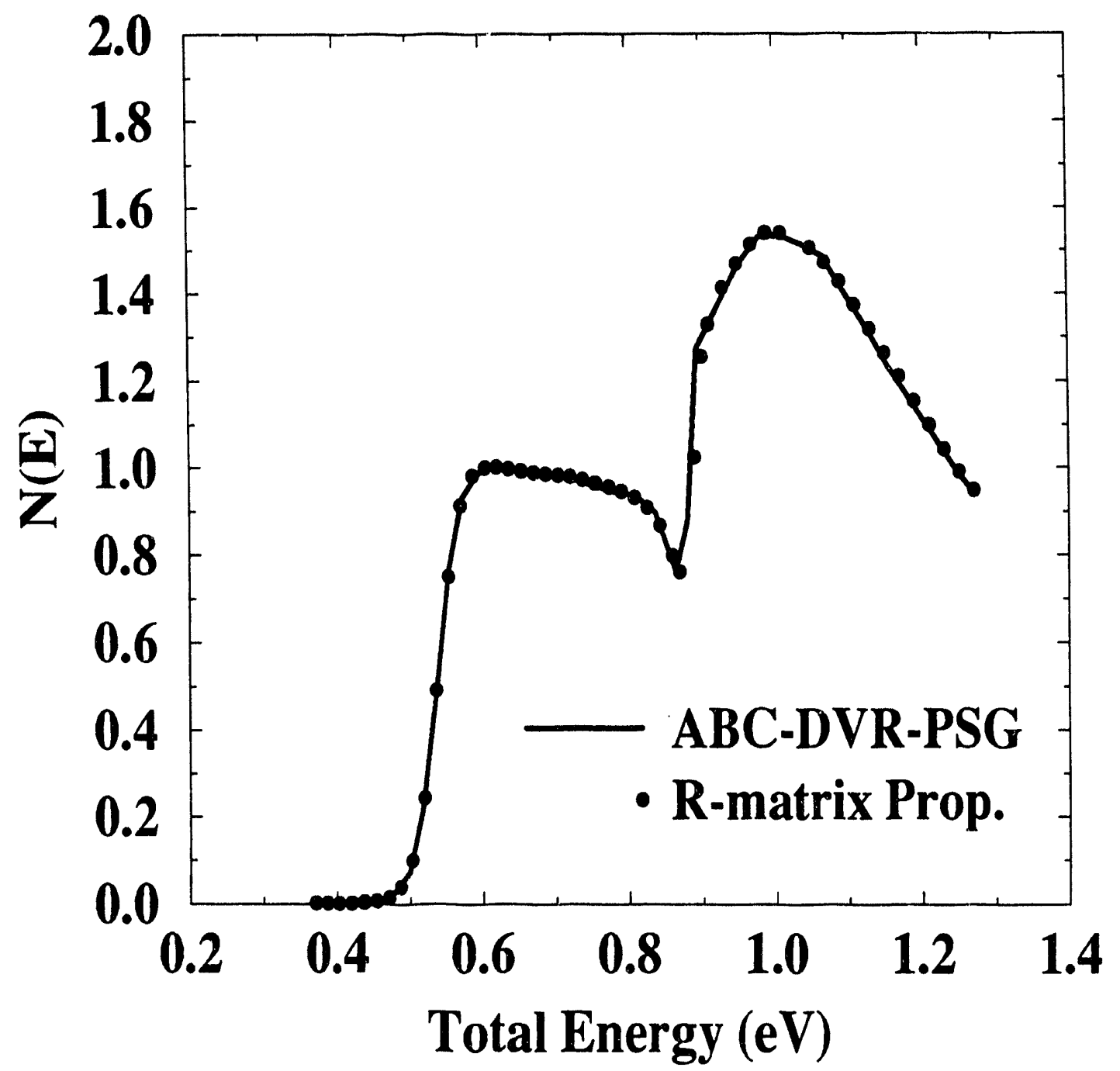

Figure 3.2: Cumulative reaction probability for the collinear $\mathrm{H}+\mathrm{H}_{2}$ reaction. The circles are the $R$-matrix propagation results (summed over final states) by Bondi et al., and the line is the power series Green's function results. Excellent agreement is obtained over the entire energy range. The "staircase" structure is evident, with $N(E) \cong 1$ when the is one open channel, and $\cong 1.6$ with two open channels. The non-monotonic increase indicates recrossing dynamics, and the peak at $E=0.87 \mathrm{eV}$ indicates a collision complex for $\mathrm{H}_{3}$. 


\subsubsection{Convergence Tests}

The two important convergence parameters introduced by the power series expansion of the ABC Green's function are the total propagation time $T$, and the time step $\Delta t$. The total time $T$ represents the time required for reaction and absorption by $\epsilon(\mathbf{q})$. The time step $\Delta t$ is the duration for which the STP is a faithful representation of the propagator. These are both a function of the dynamics and the choice of absorbing potential. We measure $\Delta t$ in units of a fundamental small time given by

$$
\tau_{\text {grid }} \equiv \frac{\text { min grid length }}{\max \text { grid velocity }}=\frac{m \Delta x^{2}}{\pi \hbar}
$$

That is, $\Delta t=f \tau_{\text {grid }}$ where $f$ is some unitless number. If we choose $\Delta x$ by the criterion in Eq. (3.40), we also find that

$$
\tau_{\text {grid }}=\frac{2 \pi \hbar}{E} \frac{1}{n_{B}^{2}} \cong \frac{2 \pi \hbar}{E} \frac{1}{16}
$$

where $n_{B}$ is defined in Eq. (3.40). Equation (3.44) shows that this fundamental small time should, at least, be small enough to integrate the energy dependent oscillations in the time integral. Whether it is small enough for propagation, i.e. whether $f$ is of order unity for convergence, is discussed below.

In the context of calculating $N(E)$ by the ABC-DVR approach, the present work represents a new iterative method to invert a complex matrix with less memory required than GMRES [48, 51]. The overall computational framework, i.e. Eq. (3.13), is not new and is not what is being tested here. As such, we gauge the error of the power series expansion in the following way. First we converge $N(E)$ using the direct ABC-DVR method. Then, using the same Hamiltonian and grid parameters, we compute the PSG result. Relative error is therefore defined as

$$
\text { error } \equiv \frac{N^{P S G}(E)-N^{\text {Direct }}(E)}{N^{\text {Direct }}(E)} \times 100 \%
$$

where the convergence of the dire:t result is based on the calculations of Bondi et al. $[103,104]$.

First we study the convergence of $N(E)$ at $E=0.52 \mathrm{eV}$. We use a grid and absorbing potential $(\lambda=1.1 \mathrm{eV}$ and $\eta=0.27 \mathrm{au})$ for which the direct ABC-DVR 
$N(E)=0.242$, whereas the $R$-matrix propagation gives $N(E)=0.244$. Figure 3.3 shows the percent error of $N(E)$ from the direct result as a function of the common logarithm of $f$ where $\tau_{\text {grid }}=20.2 a u=0.49 f s$. We see very well behaved, monotonic convergence to the direct result. This is remarkable for the following reason. As the time step is made smaller in Fig. 3.3, the exact STP becomes more oscillatory and hence, more difficult to integrate. However, the SDVR of the STP is sufficiently well behaved that it can be integrated with the same DVR grid while changing the time step by three orders of magnitude. Taking the first result that remains in the $1 \%$ error band as converged, $f=0.3$ or $\Delta t=6 a u=0.15 f s$ is the optimal time step for the STP. This time step is about $1 / 50$ of the asymptotic $\mathrm{H}_{2}$ ground state vibrational period, which is approximately $8 \mathrm{fs}$ [105]. Such a small time step is necessary to obtain accurate results for a large value of $T$.

Figure 3.4 shows the convergence of $N(E)$ to the direct result for $E=0.52$ $e V$ as a function of the total propagation time $T$. For the calculations in the solid curve, we set $f=0.05$ or $\Delta t=1 a u=0.024 \mathrm{fs}$. For those in the dashed curve $f=$ 1 or $\Delta t=20.2 a u=0.49 \mathrm{fs}$. Both curves show the same very smooth convergence. In fact, since the rate of convergence with respect to the total time $T$ is independent of the time step $\Delta t$, optimization of the two temporal parameters can be performed independently. Using the same $1 \%$ convergence criterion above, we see the optimal $T=5000 a u=121 \mathrm{fs}$. With these parameters the PSG required for $N(E)$ at $E=$ $0.52 \mathrm{eV}$ converges in roughly 800 iterations. The optimal calculation requires $c a .20$ CPU seconds on an IBM RS/6000 Model 550.

Now we consider varying parameters in the absorbing potential to effect more rapid absorption in time. Once again, consider $E=0.52 \mathrm{eV}$. At this energy, the ABCDVR cumulative reaction probability is stable over a range of $\lambda$ up to approximately $1.6 \mathrm{eV}$. At $\lambda=1.5 \mathrm{eV}, N(E)$ by direct ABC-DVR gives the value 0.245 . In Fig. 3.5 we show the same as Fig. 3.4, except with this elevated value of $\lambda$, the absorbing potential strength parameter. In this case convergence of $N(E)$ is obtained with a total propagation time $T=4000 a u=97 \mathrm{fs}$. This calculation, requiring roughly 650 iterations, represents a modest savings in computation with fairly little effort in optimization of the absorbing potential. 


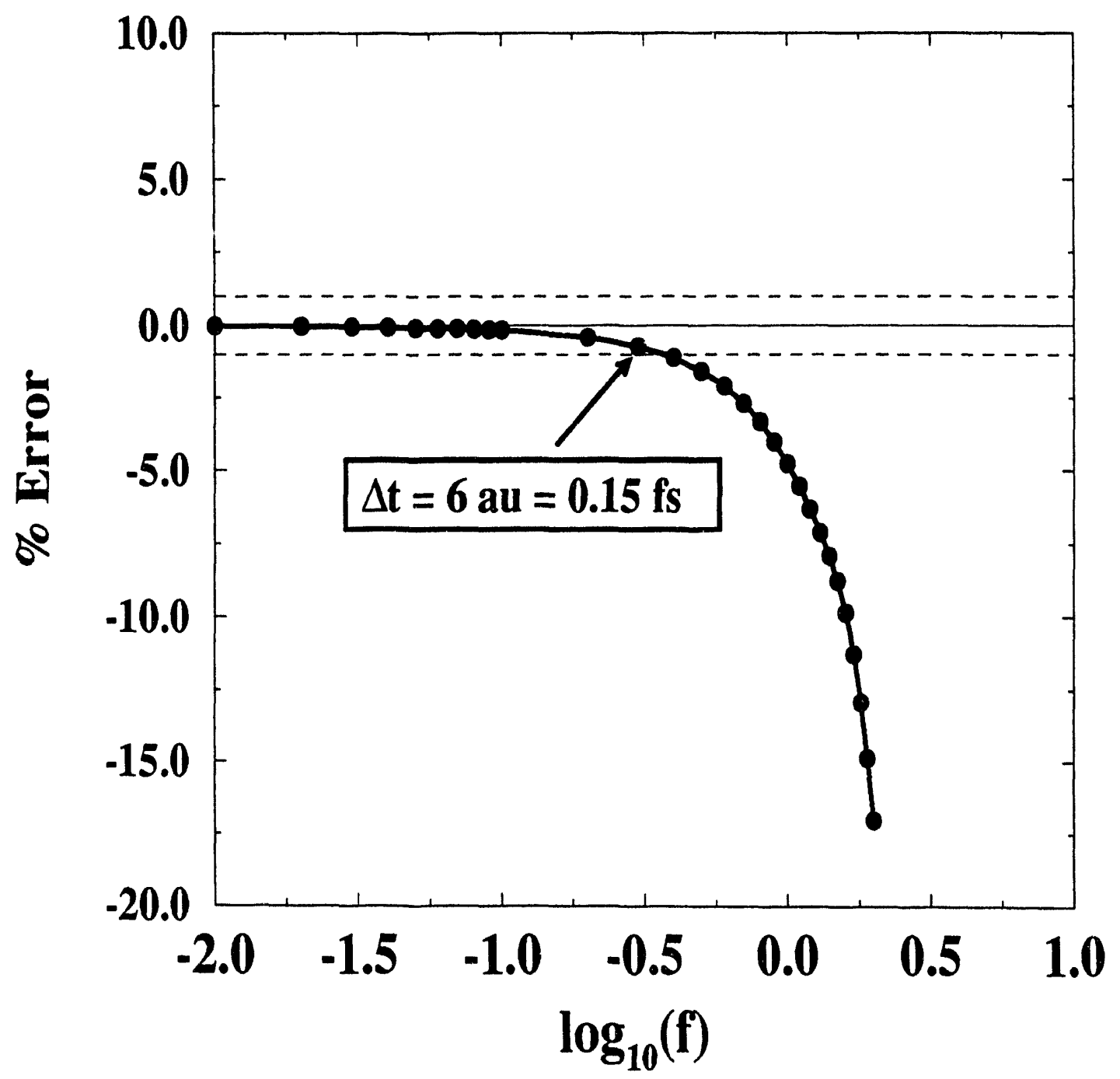

Figure 3.3: Convergence of $N(E)$ at $E=0.52 \mathrm{eV}$ with respect to the split-operator time step $\Delta t$. The abscissa is $\log _{10}(f)$ where the time step is $\Delta t=f \tau_{\text {grid }}$, with $\tau_{\text {grid }}$ $=20.2 a u=0.49 \mathrm{fs}$. Note the smooth convergence obtained with a single DVR grid, which results from the non-oscillatory effective propagator. The optimal $\Delta t=6 \mathrm{au}$ $=0.15 \mathrm{fs}$. 


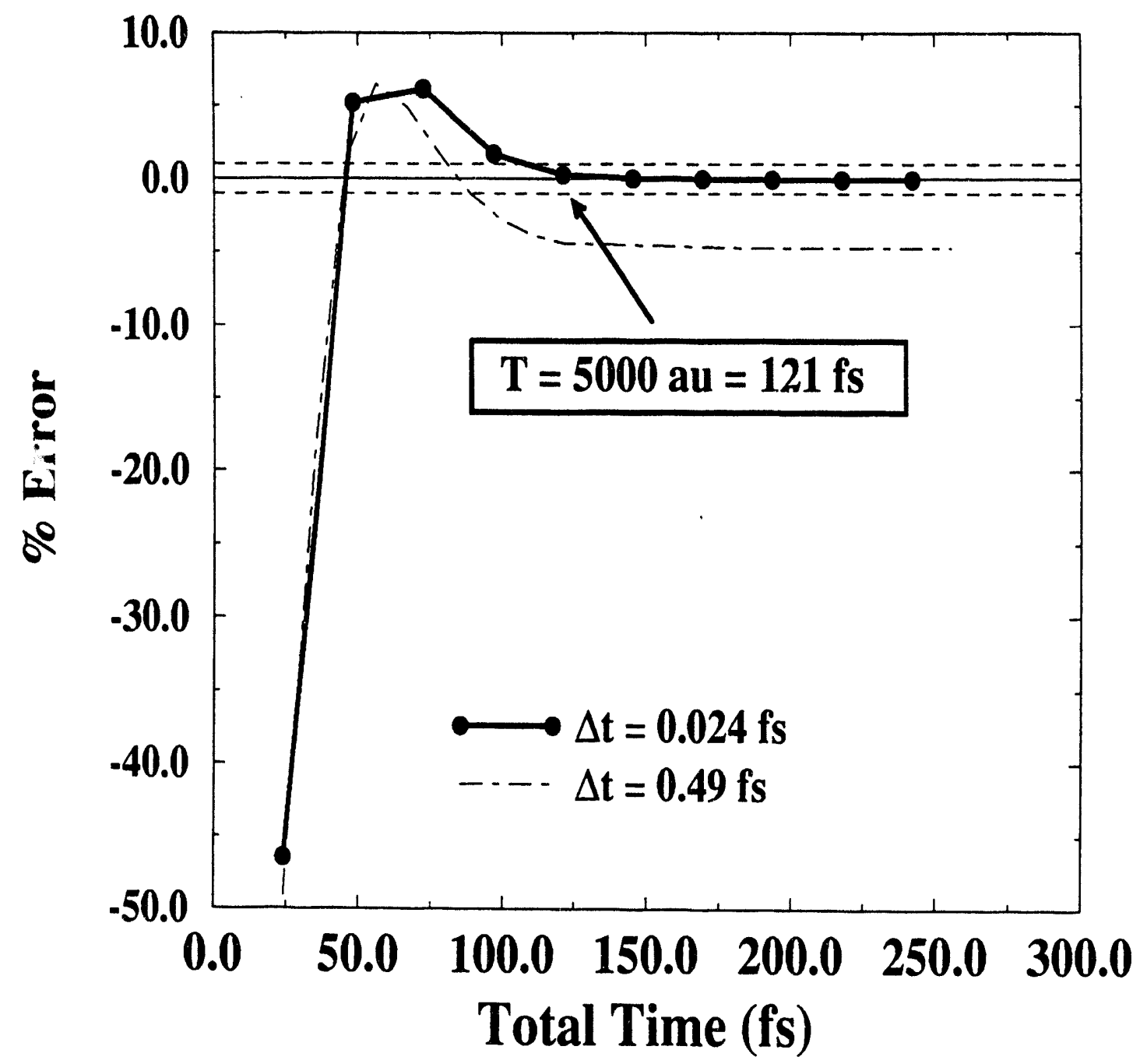

Figure 3.4: Convergence of $N(E)$ at $E=0.52 \mathrm{eV}$ with respect to the total propagation time $T$. The solid curve uses $\Delta t=1 a u=0.024 \mathrm{fs}$ and the dashed curve uses $\Delta t$ $=20.2 a u=0.49 \mathrm{fs}$. Both curves give smooth convergence, suggesting independent optimization of the parameters $\Delta t$ and $T$. The optimal $T=5000 \mathrm{au}=121 \mathrm{fs}$. 


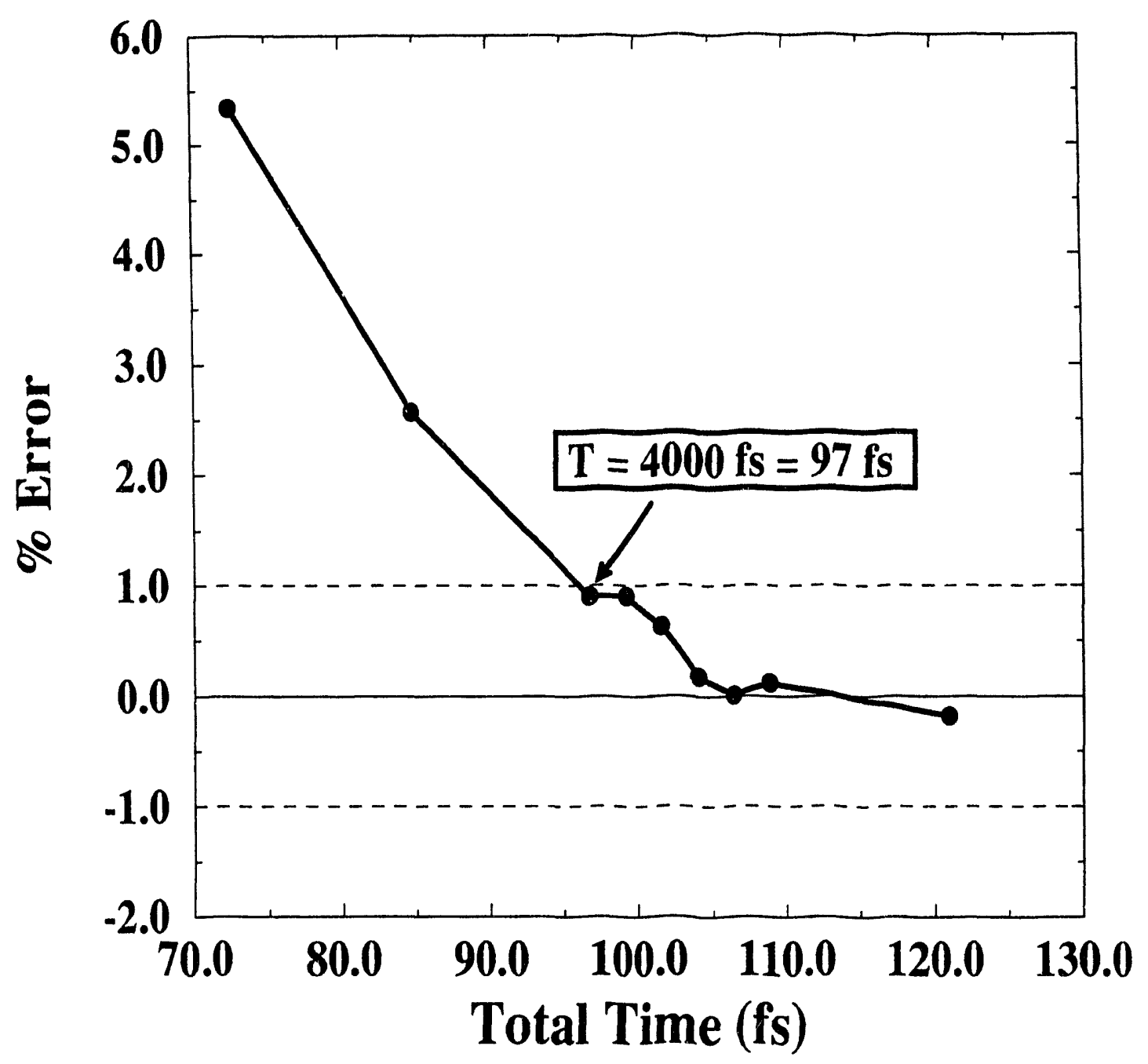

Figure 3.5: The same as Fig. 3.4, except the absorbing potential strength parameter is increased from $\lambda=1.1 \mathrm{eV}$ to $1.5 \mathrm{eV}$. In this case the optimal $T=4000 a u=97$ $f s$. The power series converges more rapidly with a stronger absorbing potential. 
Similar convergence properties were obtained at higher energies, in which more than one asymptotic vibrational channel is open. In general, $f \cong 0.3$ is sufficient with this STP to give results accurate to about $1 \%$. The total time $T$ required for convergence can be easily estimated given the size of the grid and the available translational energy. In addition, grid sizes and total propagation times can be reduced by using stronger absorbing potentials.

\subsection{Concluding Remarks}

We have described a new method to compute the absorbing boundary condition energy Green's function on a sinc-function DVR grid using a power series expansion. This is an iterative procedure for inverting the non-Hermitian matrix $(E+i \boldsymbol{\epsilon}-\mathbf{H})$ which requires very little memory. We have demonstrated the accuracy and convergence properties of the PSG method by applying it to the calculation of the cumulative reaction probability for collinear $\mathrm{H}+\mathrm{H}_{2}$. In addition, we have qualitatively discussed the numerical efficiency of SDVR relative to the FFT method. We have concluded that both approaches have their proper regime of efficient application, and in particular, that SDVR should be superior when many grid points can be deleted from the rectangular grids required for the FFT. We believe that the computation of $N(E)$ by the PSG method has the basic ingredients necessary for the realistic study of larger chemical systems. First, by computing the microcanonical reaction probability, an averaged quantity, relatively small grids can be used which focus points in the interaction region where the chemical dynamics takes place. Second, by using an iterative solution for the Green's function, we avoid having to store the Hamiltonian matrix, and can in principle treat larger systems.

There are several avenues for future study suggested by the present results. In this Section we consider the following: first, possible improvements to the iterative calculation of reaction probabilities by an ABC Green's function on a grid; and second, a generalization of Makri's effective free particle propagator. 


\subsubsection{Reactive Scattering}

The present calculation uses a uniform grid in space and time to integrate the Schrödinger equation. The uniform (spatial) grid DVR provides a simple matrix representation of the Hamiltonian operator. The uniform (temporal) grid short time propagator gives a power series expansion of the ABC Green's function. This is surely not the most sophisticated approach. We now discuss possible improvements to the method.

We conjectured above that, for the study of reactive scattering, the SDVR uniform grid would be more computationally efficient than the Fourier uniform grid because of the ease of basis truncation with SDVR. However, a non-uniform grid DVR, e.g. Gauss-Hermite DVR, would also have all the advantages that were attributed to SDVR. In addition, a non-uniform grid DVR might in principle be more efficient because it originates from adaptive quadrature. For example, the $\mathrm{H}+\mathrm{O}_{2}$ system, which has a deep well in the interaction region, is represented more efficiently in Gauss-Hermite DVR than in SDVR [106]. A uniform grid would also be very inefficient in the case of strongly exoergic systems, e.g. the $\mathrm{F}+\mathrm{H}_{2}$ reaction. In general, treating complicated systems will require that the grid be adapted to the shape of the PES. In the case of collinear $\mathrm{H}+\mathrm{H}_{2}$, which has no deep wells and is thermo-neutral, the uniform grid defined in SDVR is sufficient.

Using the trapezoidal rule and the split-operator propagator gives convergence which is second order in $\Delta t$. I.e. reducing the time step by a factor of ten gives two more converged digits in the Green's function. There are many modern methods of solving linear systems which give higher order convergence. Such rapid convergence may obviate the need to perform some of the convergence tests shown in this Chapter. As discussed briefly above (and in detail in the next Chapter), many of these high order methods utilize the Lanczos reduction algorithm. This approach is (in)famous for being highly sensitive to the effects of numerical roundoff error. Powerful methods for controlling this error have been developed for Hermitian systems, but unfortunately are much less reliable for non-Hermitian systems. Since numerical roundoff error is manifested only after several Lanczos iterations are performed, it is 
plausible that an algorithm based on a low order complex symmetric Lanczos method may be more stable.

A low order Lanczos algorithm for unitary short time propagation has been developed by Park and Light [41]. We have successfully adapted this for propagation with absorbing boundary conditions [107, 108]. However, we have found that this is not useful for the Green's function calculation. That is because the sign of the imaginary part of the complex eigenvalues tends to converge very slowly. Some of the eigenvalues have positive imaginary parts, which precludes the possibility of converging the time integral which gives the Green's function. This instability is due to additional roundoff error in the non-Hermitian calculation. To stabilize the calculation, we split off the absorbing potential from the Hermitian Hamiltonian via

$$
e^{-i(\hat{H}-i \hat{\epsilon}) t / \hbar}=e^{-\hat{\epsilon} t / 2 \hbar} e^{-i \hat{H} t / \hbar} e^{-\hat{\epsilon} t / 2 \hbar}+O\left(t^{3}\right) .
$$

This gives a stable algorithm, and is moderately efficient [108] (although not as efficient as the original split-operator based algorithm). However, this is an unacceptable compromise because the convergence is again secnnt order in $t$.

In the following Chapter, we discuss native to the Lanczos based algorithms, called the Newton polynomial expa. This is a high order method which is stable and has very straightforward accuracy control. The Newton expansion is a generalization of the well known Chebyshev expansion, and is the method of choice for the remainder of this dissertation.

\subsubsection{Path Integration}

We consider the generalization of Makri's effective free particle kernel. We will not pursue these ideas in this dissertation, but rather, offer them for contemplation. For simplicity we consider a one dimensional system with mass $m$. The success of Makri's propagator is based on the filtering of high frequency momenta which are unimportant and difficult to integrate. To this end, we denote a general momentum filter by $w(p)$ with the property that it goes to zero as $p \rightarrow \pm \infty$. This 
gives a generalized effective free particle kernel given by

$$
\left\langle x^{\prime}\left|e^{-i \hat{T} t / \hbar}\right| x\right\rangle_{e f f} \equiv \int_{-\infty}^{+\infty} \frac{d p}{2 \pi \hbar} e^{i p x^{\prime} / \hbar} e^{-i p^{2} t / 2 m \hbar} e^{-i p x / \hbar} w(p)
$$

By the convolution theorem [109] one can show that the asymptotic form of this effective kernel is given by

$$
\lim _{\tilde{p} \rightarrow \infty}\left\langle x^{\prime}\left|e^{-i \hat{T} t / \hbar}\right| x\right\rangle_{e f f}=W\left(x^{\prime}-x\right),
$$

where

$$
\tilde{p} \equiv m\left(x^{\prime}-x\right) / t
$$

and

$$
W(x)=\int_{-\infty}^{+\infty} \frac{d p}{2 \pi \hbar} e^{i p x / \hbar} w(p)
$$

is the Fourier transform of the momentum filter. In the trivial case where $w(p)=$ 1, i.e. no filter, then Eq. (3.47) gives the exact kernel. If we set $w(p)=1$ if $|p|$ $<p_{\max }$ and zero otherwise, we recover Makri's effective kernel, which we have used in a uniform grid DVR. Its asymptotic form, Eq. (3.29), is trivially obtained from Eqs. (3.48)-(3.50). Now consider the case where $w(p)$ has a Gaussian form

$$
w(p)=e^{-p^{2} / 2 \Delta p^{2}}
$$

Then the effective propagator is

$$
\left\langle x^{\prime}\left|e^{-i \hat{T} t / \hbar}\right| x\right\rangle_{\text {eff }}=\left\langle x^{\prime}\left|e^{-i \hat{T} t / \hbar}\right| x\right\rangle_{e x a c t} \times g_{\text {smooth }}\left(x^{\prime}, x, t ; m, \Delta x\right),
$$

where

$$
\begin{aligned}
g_{s m o o t h}\left(x^{\prime}, x, t ; m, \Delta x\right) & =\left\{\exp \left[\frac{-i B^{3}\left(x^{\prime}-x\right)^{2}}{A^{2}+B^{2}}\right] \exp \left[\frac{i}{2} \tan ^{-1}\left(\frac{B}{A}\right)\right]\right\} \\
& \times\left\{\left(\frac{A^{2}}{A^{2}+B^{2}}\right)^{1 / 4} \exp \left[\frac{-i A B^{2}\left(x^{\prime}-x\right)^{2}}{A^{2}+B^{2}}\right]\right\} \\
A & =1 / 2 \Delta x^{2} \\
B & =m / 2 \hbar t \\
\Delta x \Delta p & =\hbar .
\end{aligned}
$$


This effective propagator has three remarkable features. First, it is clear from Eq. (3.53) that the phase factor in $g_{s m o o t h}$ identically cancels the very rapid oscillations in the exact free particle kernel as $t$ goes to zero, i.e. $B \gg A$. This is crucial to the stability of the effective propagator. Second, the form of the filtering function, a MaxwellBoltzmann distribution, suggests that we are imposing an artificial temperature for which $k_{B} T_{f i l t e r} / 2=\Delta p^{2} / 2 m$. This is analogous to the approach of Coalson [65] in which an artificial temperature was used to compute dipole autocorrelation functions. Imposing an artificial temperature in the propagator is tantamount to propagation in complex time, which Doll [64] showed is stable even with numerical matrix multiplication (NMM). In addition, Thirumalai and Berne [63] have carried out NMM calculations of the propagator in complex time in the study of (non-artificial) temperature dependent correlation functions. Using $\Delta p=p_{\max }$ for the $N(E)$ calculations at $E=0.52 \mathrm{eV}$, the artificial temperature is $T_{f i l t e r} \cong 50,000 \mathrm{~K}$. Clearly this temperature is not low enough to interfere with the microcanonical density operator, but might be low enough to filter out the high momentum components which are unimportant to the dynamics.

Finally, an approximation to the coordinate propagator containing the same real Gaussian as in Eq. (3.53) has been previously obtained in the context of "distributed approximating functions" by Kouri, Hoffman and co-workers [74-76]. They were also seeking a more well behaved coordinate free particle propagator. To this end, they analytically propagated a Hermite polynomial fit to an initial wave packet, and observed what effective propagator would have evolved the exact wavepacket to obtain the same result. It is clear, from the present analysis, that their approach is tantamount to filtering out high frequency components in the propagator from the start, without the need to fit an initial wavepacket. The intriguing aspect of this propagator, as Kouri, Hoffman and co-workers correctly point out [74-76], is the possibility of performing a path integral Monte Carlo evaluation of the real time propagator using the real Gaussian in Eq. (3.53) as the sampling distribution. However, there are some potential problems that may arise from this strategy. First, importance sampling based on free particle dynamics may require very small time slices, and may not be able to anticipate the details of long time dynamics. Second, 
a Gaussian filter, which is characterized by only one parameter, may not be flexible enough to damp the high momentum components while giving the small components unit weight. Nevertheless, the possible success of this approach bears further thought and numerical testing.

There is another perspective with which we can generalize Makri's effective propagator. Most studies of path integration begin with an exact propagator for some reference system, including the coupling with the split-operator expression. By using an exact reference propagator, infinite frequencies have implicitly been included, which then have to be filtered. The art is in constructing a convenient filter which does not also filter out the interesting dynamics.

On the other hand, conventional basis set quantum calculations begin with very low frequencies, and try to build in higher frequencies if necessary and if possible. The finite basis set already has an implict "filter," in that only finite frequencies are included. That these two approaches can be equivalent was demonstrated in this Chapter.

To build on this idea, we consider a Hamiltonian $\mathbf{H}$ partitioned as $\mathbf{H}=\mathbf{H}_{0}$ $+\mathbf{H}_{1}$, where $\mathbf{H}_{0}$ is a simple reference system whose eigenvalues $\left\{\varepsilon_{n}\right\}$ and eigenvectors $\left\{\mathbf{v}_{n}\right\}$ are known. The propagator is then approximated as:

$$
\begin{aligned}
e^{-i \mathbf{H} t / \hbar} & \cong e^{-i \mathbf{H}_{0} t / 2 \hbar} e^{-i \mathbf{H}_{1} t / \hbar} e^{-i \mathbf{H}_{0} t / 2 \hbar} \\
& \cong \sum_{n^{\prime}=1}^{N} \sum_{n=1}^{N} \cdot \mathbf{v}_{n^{\prime}} e^{-i \varepsilon_{n^{\prime}} t / 2 \hbar}\left[\mathbf{v}_{n^{\prime}}^{\dagger} \cdot e^{-i \mathbf{H}_{1} t / \hbar} \cdot \mathbf{v}_{n}\right] e^{-i \varepsilon_{n} t / 2 \hbar} \mathbf{v}_{n}^{\dagger}
\end{aligned}
$$

where the first approximation is in the splitting, and the second is in the finite basis representation. The use of a finite basis automatically filters out high frequencies. This is precisely the form used in the present Chapter, where $\mathbf{H}_{0}=\mathbf{V}-i \boldsymbol{\epsilon}$ and $\mathbf{H}_{1}=$ T. Furthermore, the eigenvectors are DVR states, and the eigenvalues are $V_{n}-i \epsilon_{n}$. Equation (3.54) offers the possibility of using a different, more adapted reference system. For example, a vibrational problem may be better represented using harmonic oscillator states than free particle states. The key is not to use the exact harmonic oscillator propagator, but rather, its finite basis approximation. Topaler and Makri [110] use this approach as their method of choice in path integral calculations of 
the rate constant flux autocorrelation function and of the intramolecular vibrational relaxation wavefunction correlation function.

\section{References}

[1] W. H. Miller, J. Chem. Phys. 62, 1899 (1975).

[2] T. Yamamoto, J. Chem. Phys. 33,281 (1960).

[3] W. H. Miller, J. Chem. Phys. 61, 1823 (1974).

[4] W. H. Miller, S. D. Schwartz, and J. W. Tromp, J. Chem. Phys. 79, 4889 (1983).

[5] R. Jaquet and W. H. Miller, J. Phys. Chem. 89, 2139 (1985).

[6j K. Yamashita and W. H. Miller, J. Chem. Phys. 82, 5475 (1985).

[7] J. W. Tromp and W. H. Miller, J. Phys. Chem. 90, 3482 (1986).

[8] J. W. Tromp and W. H. Miller, Faraday Discuss. Chem. Soc. 84, 441 (1987).

[9] R. E. Wyatt, Chem. Phys. Lett. 121, 301 (1985).

[10] T. J. Park and J. C. Light, J. Chem. Phys. 88, 4897 (1988); 91, 974 (1989).

[11] M. Founargiotakis and J. C. Light, J. Chem. Phys. 93, 633 (1990).

[12] T. J. Park and J. C. Light, J. Chem. Phys. 94, 2946 (1991); 96, 8853 (1992).

[13] G. Wahnstrom and H. Metiu, Chem. Phys. Lett. 134, 531 (1987); J. Phys. Chem. 92, 3240 (1988).

[14] G. Wahnstrom, B. Carmeli, and H. Metiu, J. Chem. Phys. 88, 2478 (1988).

[15] A. E. Depristo, K. Haug, and H. Metiu, Chem. Phys. Lett. 155, 376 (1989).

[16] R. Lefebvre and N. Moiseyev, J. Chem. Phys. 93, 7173 (1990). 
[17] N. Ron, N. Moiseyev, and R.Lefebvre, J. Chem. Phys. 96, 8307 (1992).

[18] P. N. Day and D. G. Truhlar, J. Chem. Phys. 94, 2045 (1991).

[19] D. Thirumalai, B. C. Garrett, and B. J. Berne, J. Chem. Phys. 83, 2972 (1985).

[20] C. W. McCurdy and B. C. Garrett, J. Chem. Phys. 84, 2630 (1986).

[21] T. Seideman and W. H. Miller, J. Chem. Phys. 95, 1768 (1991).

[22] E. N. Economou, Green's Functions in Quantum Physics (Springer, Berlin, 1990), p. 7.

[23] T. Seideman and W. H. Miller, J. Chem. Phys. 96, 4412 (1992); 97, 2499 (1992).

[24] A. Goldberg and B. W. Shore, J. Phys. B 11, 3339 (1978).

[25] C. Leforestier and R. E. Wyatt, J. Chem. Phys. 78, 2334 (1983).

[26] R. Kosloff and D. Kosloff, J. Comput. Phys. 63, 363 (1986).

[27] D. Neuhauser and M. Baer, J. Chem. Phys. 90, 4351 (1989).

[28] W. H. Press, B. P. Flannery, S. A. Teukolsky, and W. T. Vetterling, Numerical Recipes: The Art of Scientific Computing (Cambridge University Press, Cambridge, 1986).

[29] D. M. Young, Comput. Phys. Commun. 53, 1 (1989).

[30] C. Lanczos, J. Res. Natl. Bur. Stand. 45, 255 (1950).

[31] J. K. Cullum and R. A. Willoughby, Lanczos Algorithm for Large Symmetric Eigenvalue Computations Vol. 1, Theory (Birkhäuser, Boston, 1985).

[32] C. C. Paige and M. A. Saunders, SIAM J. Numer. Anal. 12, 617 (1975).

[33] A. Nauts and R. E. Wyatt, Phys. Rev. Lett. 51, 2238 (1983).

[34] N. Moiseyev, R. A. Friesner, and R. E. Wyatt, J. Chem. Phys. 85, 331 (1986). 
[35] F. Le Quéré and C. Leforestier, J. Chem. Phys. 92, 247 (1990).

[36] G. C. Groenenboom and H. M. Buck, J. Chem. Phys. 92, 4374 (1990).

[37] G. C. Groenenboom, Report, Netherlands Organization for Scientific Research (NWO), 1992.

[38] G. C. Groenenboom and D. T. Colbert, J. Chem. Phys. 99, 0000 (1993).

[39] W. T. Yang and W. H. Miller, J. Chem. Phys. 91, 3504 (1989).

[40] W. T. Yang and A. C. Peet, J. Chem. Phys. 92, 522 (1990).

[41] T. J. Park and J. C. Light, J. Chem. Phys. 85, 5870 (1986).

[42] U. Manthe, H. Köppel, L. S. Cederbaum, J. Chem. Phys. 95, 1708 (1991).

[43] S. K. Gray, J. Chem. Phys. 96, 6543 (1992).

[44] G. Moro and J. H. Freed, J. Chem. Phys. 74, 3757 (1981).

[45] W. A. Wassam, J. Chem. Phys. 82, 3371 (1985); 82, 3386 (1985).

[46] J. J. Jiang and J. S. Hutchinson, J. Chem. Phys. 93, 5643 (1990).

[47] S. Dallwig, N. Fahrer, and C. Schlier, Chem. Phys. Lett. 191, 69 (1992).

[48] Y.Saad and M. H. Schultz, Report RR-254, Dept. of Comp. Sci., Yale University (1983); SIAM J. Sci. Stat. Comput. 7, 856 (1986).

[49] C. Duneczky, R. E. Wyatt, D. Chatfield, K. Haug, D. W. Schwenke, D. G. Truhlar, Y. Sun, and D. J. Kouri, Comput. Phys. Commun. 53, 357 (1989).

[50] D. E. Manolopoulos, R. E. Wyatt, and D. C. Clary, J. Chem. Soc. Faraday Trans. 86, 1641 (1990).

[51] Y. Saad, J. Comp. Appl. Math. 24, 89 (1988). 
[52] A brief summary of the power series Green's function appeared previously in the following article:

S. M. Auerbach and W. H. Miller, J. Chem. Phys. 98, 6917 (1993).

[53] D. O. Harris, G. G. Engerholm, and W. D. Gwinn, J. Chem. Phys. 43, 1515 (1965).

[54] A. S. Dickinson and P. R. Certain, J. Chem. Phys. 49, 4209 (1968).

[55] J. C. Light, I. P. Hamilton, and J. V. Lill, J. Chem. Phys. 82, 1400 (1985).

[56] D. T. Colbert and W. H. Miller, J. Chem. Phys. 96, 1982 (1992).

[57] J. Z. H. Zhang, S.-I. Chu, and W. H. Miller, J. Chem. Phys. 88, 6233 (1988).

[58] J. Z. H. Zhang and W. H. Miller, J. Chem. Phys. 91, 1528 (1989).

[59] Z. Bačić and J. C. Light, J. Chem. Phys. 85, 4594 (1986).

[60] E. A. McCullough and R. E. Wyatt, J. Chem. Phys. 54, 3578 (1971).

[61] R. Kosloff, J. Phys. Chem. 92, 2087 (1988) and references therein.

[62] D. Kosloff and R. Kosloff, J. Comput. Phys. 52, 35 (1983).

[63] D. Thirumalai and B. Berne, J. Chem. Phys. 81, 2512 (1984).

[64] J. Doll, J. Chem. Phys. 81, 3536 (1984).

[65] R. D. Coalson, J. Chem. Phys. 83, 688 (1985).

[66] B. J. Berne and D. Thirumalai, Annu. Rev. Phys. Chem. 37, 401 (1986).

[67] P. G. Wolynes, J. Chem. Phys. 87, 6559 (1987).

[68] J. Doll, D. L. Freeman, and M. J. Gillan, Chem. Phys. Lett. 143, 277 (1988).

[69] N. Makri, Chem. Phys. Lett. 159, 489 (1989).

[70] J. S. Bader, R. A. Kuharski, and D. Chandler, J. Chem. Phys. 93, 230 (1990). 
[71] C. H. Mak and D. Chandler, Phys. Rev. A 41, 5709 (1990); 44, 2352 (1991).

[72] C. H. Mak, Phys. Rev. Lett. 68, 899 (1992).

[73] O. A. Sharafeddin, D. J. Kouri, N. Nayar, and D. K. Hoffman, J. Chem. Phys. 95, 3224 (1991).

[74] D. K. Hoffman, N. Nayar, O. A. Sharafeddin, and D. J. Kouri, J. Phys. Chem. 95, 8299 (1991).

[75] D. K. Hoffman and D. J. Kouri, J. Phys. Chem. 96, 1179 (1992).

[76] D. K. Hoffman, M. Arnold, and D. J. Kouri, J. Phys. Chem. 96, 6539 (1992).

[77] N. Metropolis, A. W. Rosenbluth, M. N. Rosenbluth, H. Teller, and E. Teller, J. Chem. Phys. 21, 1087 (1953).

[78] R. P. Feynman, Rev. Mod. Phys. 20, 367 (1948).

[79] L. S. Rodberg and R. M. Thaler, Introduction to the Quantum Theory of Scattering (Academic Press, New York, 1967), pp. 73-183.

[80] An alternative representation of the trace which is preferable for both conceptual and computational reasons has recently been reported in U. Manthe and W. H. Miller, J. Chem. Phys. 99, 3411 (1993).

This involves evaluating the trace by obtaining the largest eigenvalues of the "reaction probability operator" $\hat{P} \equiv \hat{G}_{A B C}^{*}(E) \hat{\epsilon}_{p} \hat{G}_{A B C}(E) \hat{\epsilon}_{r}$. These eigenvalues are "eigen reaction probabilities," which are the rigorous generalization of those arising from a separable transition state theory approximation for $N(E)$.

[81] N. Makri, Comput. Phys. Commun. 63, 389 (1991).

[82] C. Leforestier, R. H. Bisseling, C. C $\epsilon_{i j a n}$, M. D. Feit, R. Friesner, A. Guldberg, A. Hammerich, G. Jolicard, W. Karrlein, H.-D. Meyer, N. Lipkin, O. Roncero, and R. Kosloff, J. Comput. Phys. 94, 59 (1991). 
[83] T. N. Truong, J. J. Tanner, P. Bala, J. A. McCammon, D. J. Kouri, B. Lesyng, and D. K. Hoffman, J. Chem. Phys. 96, 2077 (1992).

[84] J. A. Fleck, J. R. Morris, and M. D. Feit, Appl. Phys. 10, 129 (1976).

[85] M. D. Feit, J. A. Fleck, and A. Steiger, J. Comput. Phys. 47, 412 (1982).

[86] M. D. Feit and J. A. Fleck, J. Chem. Phys. 80, 2578 (1984).

[87] A. D. Bandrauk and H. Shen, Chem. Phys. Lett. 176, 428 (1991).

[88] D. Yevick, M. Glasner, and B. Hermansson, Opt. Lett. 17, 725 (1992).

[89] J. Gomezordonez and M. Morillo, Physica A 183, 490 (1992).

[90] R. N. Barnett, U. Landman, S. Dhar, N. R. Kestner, J. Jortner, and A. Nitzan, J. Chem. Phys. 91, 7797 (1989).

[91] R. B. Gerber and R. Alimi, Isr. J. Chem. 31, 383 (1991).

[92] E. Yurtsever and J. Brickmann, Int. J. Phys. Chem. 96, 142 (1992).

[93] See for example

S. K. Gray and C. E. Wozny, J. Chem. Phys. 91, 7671 (1989).

A. Untch, K. Weide, and R. Schinke, J. Chem. Phys. 95, 6496 (1991).

[94] For an $F$-dimensional system with $n$ points per degree of freedom, the SDVR prefactor is $n / \log _{2} n$. For $n=15$, the SDVR prefactor is about 4 , i.e. of order unity.

[95] For an $F$-dimensional system with $n$ points per degree of freedom, the Fourier method prefactor is of order unity, and depends on whether $n$ is strictly a power of two.

[96] R. S Mowrey, J. Chem. Phys. 94, 7098 (1991). 
[97] The $n^{\text {th }}$ term in the sum involves $\left\{\cos \left[\pi n\left(j^{\prime}-j\right) / N\right]-\cos \left[\pi n\left(j^{\prime}+j\right) / N\right]\right\}$. In the infinite $N$ limit, the former term gives the integral in Eq. (3.24), whereas the latter becomes infinitely oscillatory and gives no contribution to the matrix element.

[98] M. Abramowitz and I. A. Stegun, Handbook of Mathematical Functions with Formulas, Graphs, and Mathematical Tables (John Wiley and Sons, New York, 1972), p. 46.

[99] D. Thirumalai, E. J. Bruskin, and B. J. Berne, J. Chem. Phys. 79, 5063 (1983).

[100] P. Siegbahn and B. Liu, J. Chem. Phys. 68, 2457 (1978).

[101] D. G. Truhlar and C. J. Horowitz, J. Chem. Phys. 68, 2566 (1978); 71, 1514(E) (1979).

[102] E. B. Wilson, J. C. Decius, and P. C. Cross, Molecular Vibrations (McGraw-Hill, New York, 1955).

[103] D. K. Bondi, D. C. Clary, J. N. L. Connor, B. C. Garret, and D. G. Truhlar, J. Chem. Phys. 76, 4986 (1982).

[104] D. K. Bondi and J. N. L. Connor, J. Chem. Phys. 82, 4383 (1985).

[105] G. Herzberg, Molecular Spectra and Molecular Structure I. Spectra of Diatomic Molecules D. Van Nostrand Co., Inc, Princeton, 1967).

[106] D. T. Colbert, private communication.

[107] Please see Eqs. (5.1) and (5.2) of Ref. 52 for more details of the Lanczos propagator applied to the ABC Green's function.

[108] S. M. Auerbach, unpublished work.

[109] G. Arfken, Mathematical Methods for Physicists (Academic Press, Inc., San Diego, 1985).

[110] M. Topaler and N. Makri, Chem. Phys. Lett. 210, 448 (1993). 


\section{Chapter 4}

\section{The Newton Algorithm}

\subsection{Introduction}

In the previous Chapter, we described a new computational approach for reactive scattering calculations on large systems. There are two basic ingredients in this approach. First, the use of absorbing boundary conditions (ABC) to compute directly averaged reaction probabilities allows numerically exact scattering calculations while only sampling the localized chemical interaction region. Second, the use of a grid basis coupled with iterative methods provides the solution to the $A B C$ system without the need to store the Hamiltonian matrix. The relatively small and sparse Hamiltonian matrix which results from these two components is crucial to extending theory to larger systems. In the present Chapter, we extend both of these aspects, yielding a powerful computational algorithm for treating the reactivity of complex chemical systems.

In Chapter 3 we focused on the cumulative reaction probability, using the $A B C$ formulation of quantum reactive scattering theory. As emphasized, the calculation of averaged reaction probabilities should be more economical, and thus more applicable to larger chemical systems. However, many revealing measurements of chemical reactivity involve scattering cross sc tions $[1,2]$, which involve reaction probabilities weighted by the initial translational energy [3]. Thus, to compute cross sections, we need reaction probabilities with initial state selection. In this Chapter, 
we derive the $A B C$ formulation of the initial state selected reaction probability [4]. In addition, we demonstrate that this quantity is obtained more economically than the state-to-state probability, while offering interesting details about the chemical reaction.

The direct calculation of the initial state selected reaction probability relies on the ABC Green's function. We proposed an iterative method in Chapter 3 called the power series Green's function (PSG). In the present Chapter we extend this method for two reasons. First, the short time propagator implicit in the PSG requires optimization of the time step. Although we showed that this optimization gives smooth convergence duc to filtering out high momentum components, a superior method would avoid the need for these convergence tests. Second, the split operator short time propagator is based on exponentiating the kinetic energy. While this is straightforward for rectilinear coordinate systems, it is extremely inconvenient for curvilinear kinetic energy terms such as Coriolis coupling. Thus, we seek an iterative calculation of the ABC Green's function which is more automated and flexible than the PSG, for use on complex systems.

In the field of time dependent wavepacket propagation, a method exists with many of the attributes we seek. The Chebyshev polynomial expausion [5] of the unitary time propagator is extremely accurate, easy to automate, and only requires applying the Hamiltonian on a vector. This expansion has been used extensively to study photodissociation, gas surface scattering, and gas phase reactive scattering [6]. Inspired by the success of the Chebyshev algorithm, we are led to ask: Does an analogous polynomial expansion exist for the ABC Green's function? Much of this Chapter is devoted to answering this question.

We derive a convenient and well behaved expression for the initial state reaction probability in the $\mathrm{ABC}$ formulation. After analyzing various polynomial expansions for the ABC Green's fucntion, we discover that no direct expansion yields a tractable algorithm for large systems. We eventually advocate a method, called the Newton algorithm, which is quite similar in spirit to the PSG. However, the Newton algorithm is a vast improvement over the PSG, providing the flexibility and convenience of the Chebyshev method. We illustrate the Newton algorithm on the 
calculation of three dimensional reaction probabilities for the $\mathrm{D}+\mathrm{H}_{2}(v, j) \rightarrow \mathrm{DH}+\mathrm{H}$ initial state selected reaction. These calculations demonstrate that the Newton algorithm is capable of arbitrary accuracy with a very modest amount of computational effort [7].

\subsection{Quantum Reactive Scattering Formulation}

We outline the formal scattering theory required to define a direct calculation of the IRP. In addition, the ABC modifications to the formal theory are discussed in this Section.

\subsubsection{Formal Theory}

The IRP at total energy $E$ is defined by

$$
P_{\mathbf{n}_{r}}(E) \equiv \sum_{\mathbf{n}_{p}} P_{\mathbf{n}_{p}, \mathbf{n}_{r}}(E)
$$

where $P_{\mathbf{n}_{p}, \mathbf{n}_{r}}(E)$ is the state-to-state reaction probability from reactant state $\mathbf{n}_{r}$ to product state $\mathbf{n}_{p}$. This quantity has been of interest to many groups, most recently Neuhauser and Baer [8] and Thompson and Miller [4], because it is less computationally intensive than the fully state-selected reaction probability $P_{\mathbf{n}_{p}, \mathbf{n}_{r}}(E)$. Bacod on the analysis of the reactive flux operator $\hat{F}$ (see below), Miller [9] has shown that the IRP can be obtained directly via

$$
P_{\mathbf{n}_{r}}(E)=\left\langle\Psi_{\mathbf{n}_{r}}^{+}(E)|\hat{F}| \Psi_{\mathbf{n}_{r}}^{+}(E)\right\rangle
$$

where $\left|\Psi_{\mathbf{n}_{r}}^{+}(E)\right\rangle$ is a scattering eigenstate of the Hamiltonian operator $\hat{H}=\hat{K}+\hat{V}$. As such, the problem is reduced to determining the scattering wavefunction. Although at first sight this seems like very little progress, below we show that the use of $A B C$ allows us to accurately compute the IRP without a complete determination of the wavefunction. In the present study, as well as in Ref. 4, we evaluate $\left|\Psi_{\mathbf{n}_{r}}^{+}(E)\right\rangle$ with an integral equation. For the purpose of seeing how to correctly use $\mathrm{ABC}$, it is instructive to show the derivation of the integral equation we use. 


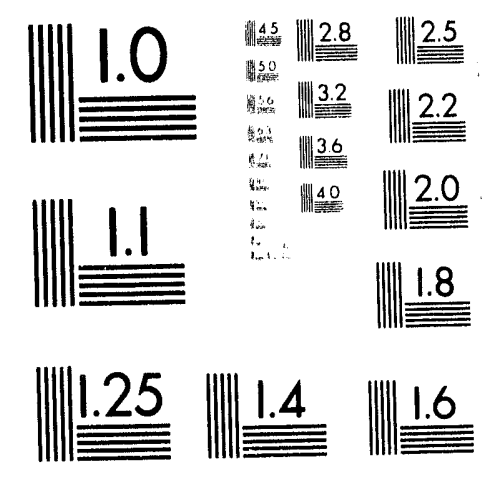

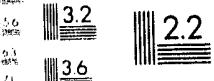

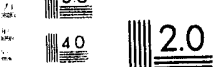



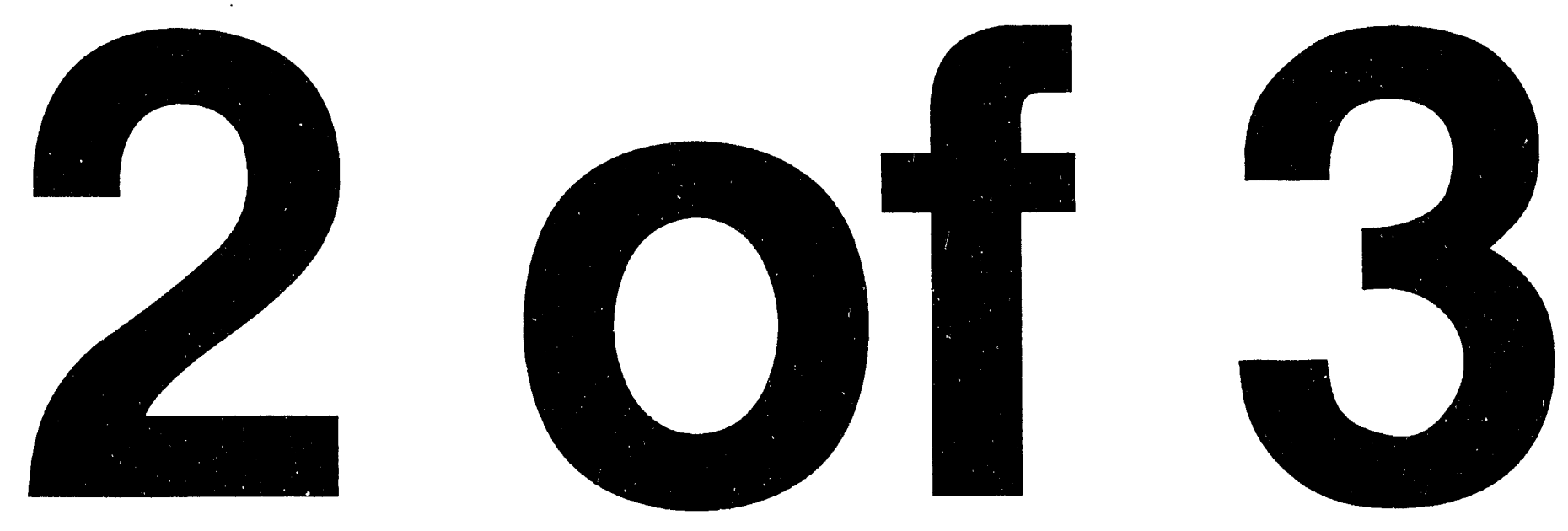
We consider a one dimensional radial system with coordinate $R$. The multidimensional generalization is extremely straightforward. The time independent Schrödinger equation for a scattering system at energy $E$ with outgoing waves (denoted by "+") in all open channels and an incoming wave in reactant channel $\mathbf{n}_{r}$ is given by:

$$
(E-\hat{H})\left|\Psi_{\mathbf{n}_{r}}^{+}(E)\right\rangle=0 .
$$

The state ket can be written in integral equation form via the Lippmann-Schwinger equation [3]

$$
\left|\Psi_{\mathbf{n}_{r}}^{+}(E)\right\rangle=\left|\Phi_{\mathbf{n}_{r}}(E)\right\rangle+\hat{G}_{0}^{+}(E) \hat{V}\left|\Psi_{\mathbf{n}_{r}}^{+}(E)\right\rangle
$$

where $\hat{G}_{0}^{+}(\llcorner)$is the unperturbed Green's function given by

$$
\hat{G}_{0}^{+}(E) \equiv \lim _{\epsilon \rightarrow 0^{+}}(E+i \epsilon-\hat{K})^{-1} .
$$

In addition, $\left|\Phi_{\mathbf{n}_{r}}(E)\right\rangle$ is the corresponding unperturbed scattering state satisfying the conditions

$$
\begin{aligned}
& (E-\hat{K})\left|\Phi_{\mathbf{n}_{r}}(E)\right\rangle=0 \\
& \lim _{R \rightarrow 0^{+}}\left\langle R \mid \Phi_{\mathbf{n}_{r}}(E)\right\rangle=0 .
\end{aligned}
$$

The first condition demonstrates that $\left|\Phi_{\mathbf{n}_{r}}(E)\right\rangle$ is indeed a free wave, and the second chooses the physically important "regular" solution.

Equation (4.4) is not immediately useful because it represents the unknown ket in terms of itself. However, it can be solved formally by the method of successive approximations [3], which is based on the fact that $\left|\Psi_{\mathbf{n}_{r}}^{+}(E)\right\rangle \cong\left|\Phi_{\mathbf{n}_{r}}(E)\right\rangle$ when $\hat{V}$ weakly perturbs $\hat{K}$. The first step, then, is to replace $\left|\Psi_{\mathbf{n}_{r}}^{+}(E)\right\rangle$ on the right hand side cf Eq. (4.4) with $\left|\Phi_{\mathbf{n}_{r}}(E)\right\rangle$, yielding the so-called first Born approximation:

$$
\left|\Psi_{\mathbf{n}_{r}}^{(1)}(E)\right\rangle=\left|\Phi_{\mathbf{n}_{r}}(E)\right\rangle+\hat{G}_{0}^{+}(E) \hat{V}\left|\Phi_{\mathbf{n}_{r}}(E)\right\rangle .
$$

Substituting the first Born wavefunction into the right hand side of Eq. (4.4) gives the second Born approximation:

$$
\begin{aligned}
\left|\Psi_{\mathbf{n}_{r}}^{(2)}(E)\right\rangle & =\left|\Phi_{\mathbf{n}_{r}}(E)\right\rangle+\hat{G}_{0}^{+}(E) \hat{V}\left|\Phi_{\mathbf{n}_{r}}(E)\right\rangle+\hat{G}_{0}^{+}(E) \hat{V} \hat{G}_{0}^{+}(E) \hat{V}\left|\Phi_{\mathbf{n}_{r}}(E)\right\rangle \\
& =\left|\Phi_{\mathbf{n}_{r}}(E)\right\rangle+\left[\hat{G}_{0}^{+}(E)+\hat{G}_{0}^{+}(E) \hat{V} \hat{G}_{0}^{+}(E)\right] \hat{V}\left|\Phi_{\mathbf{n}_{r}}(E)\right\rangle .
\end{aligned}
$$


Iterating this procedure ad infinitum yields the Born expansion for the state ket:

$$
\begin{aligned}
\left|\Psi_{\mathbf{n}_{r}}^{+}(E)\right\rangle & =\left|\Phi_{\mathbf{n}_{r}}(E)\right\rangle+\left\{\hat{G}_{0}^{+}(E) \sum_{n=0}^{\infty}\left[\hat{V} \hat{G}_{0}^{+}(E)\right]^{n}\right\} \hat{V}\left|\Phi_{\mathbf{n}_{r}}(E)\right\rangle \\
& \equiv\left|\Phi_{\mathbf{n}_{r}}(E)\right\rangle+\hat{G}^{+}(E) \hat{V}\left|\Phi_{\mathbf{n}_{r}}(E)\right\rangle
\end{aligned}
$$

Assuming convergence of the geometric expansion $\sum_{n=0}^{\infty} z^{n}=(1-z)^{-1}$ in Eq. (4.9), the fully perturbed Green's function satisfies

$$
\begin{aligned}
\hat{G}^{+}(E) & =\hat{G}_{0}^{+}(E)\left[\hat{1}-\hat{V} \hat{G}_{0}^{+}(E)\right]^{-1} \\
& =\lim _{\epsilon \rightarrow 0^{+}}(E+i \epsilon-\hat{K})^{-1}\left[\hat{1}-\hat{V}(E+i \epsilon-\hat{K})^{-1}\right]^{-1} \\
& =\lim _{\epsilon \rightarrow 0^{+}}\left\{\left[\hat{1}-\hat{V}(E+i \epsilon-\hat{K})^{-1}\right](E+i \epsilon-\hat{K})\right\}^{-1} \\
& =\lim _{\epsilon \rightarrow 0^{+}}(E+i \epsilon-\hat{H})^{-1} .
\end{aligned}
$$

Next, making the observation that

$$
\hat{V}\left|\Phi_{\mathbf{n}_{r}}(E)\right\rangle=(\hat{H}-E)\left|\Phi_{\mathbf{n}_{r}}(E)\right\rangle,
$$

the state ket becomes:

$$
\left|\Psi_{\mathbf{n}_{r}}^{+}(E)\right\rangle=\left[\hat{1}+\hat{G}^{+}(E)(\hat{H}-E)\right]\left|\Phi_{\mathbf{n}_{r}}(E)\right\rangle
$$

This is almost the final form of the desired integral equation. Before proceeding, though, we make three general points regarding Eq. (4.11).

First, there is no longer any explicit reference to the coupling term $\hat{V}$ in the Hamiltonian which is disregarded in defining $\left|\Phi_{\mathbf{n}_{r}}(E)\right\rangle$. Although the LippmannSchwinger equation defines $\left|\Phi_{\mathbf{n}_{r}}(E)\right\rangle$ as a free wave, Eq. (4.11) does not require that choice. Indeed, $\left|\Phi_{\mathbf{n}_{r}}(E)\right\rangle$ is only really required to satisfy two boundary conditions. First, it is required to be an eigenstate of $\hat{H}$ with energy $E$ for large $R$, i.e. when the colliding particles are well separated. Second, $\left|\Phi_{\mathbf{n}_{r}}(E)\right\rangle$ is regular at the origin, i.e. it is required to vanish in the limit that the colliding particles coincide.

The second point is that Eq. (4.11) allows us to split our effort in determining the reaction probabilities. That is, the closer $\left|\Phi_{\mathbf{n}_{r}}(E)\right\rangle$ is to $\left|\Psi_{\mathbf{n}_{r}}^{+}(E)\right\rangle$, the less dynamical information is required from $\hat{G}^{+}(E)$. We can see this by considering a two dimensional reactive system with reactant/product translational coordinates $R_{r} / R_{p}$. The reactant interaction region is where $\chi_{\mathbf{n}_{r}}\left(R_{r}, E\right) \equiv\left\langle R_{r}|\hat{H}-E| \Phi_{\mathbf{n}_{r}}(E)\right\rangle \neq 0$. 
Clearly, $\chi_{\mathbf{n}_{r}}\left(R_{r}, E\right)$ must vanish in the limits of small and large $R_{r}$. The same applies for the product interaction region defined by the function $\chi_{\mathbf{n}_{p}}\left(R_{p}, E\right)$. In terms of these quantities, the reactive transition amplitude $T_{\mathbf{n}_{p}, \mathbf{n}_{r}}(E)$ is given by:

$$
\begin{aligned}
T_{\mathbf{n}_{p}, \mathbf{n}_{r}}(E) & \equiv\left\langle\Phi_{\mathbf{n}_{p}}(E)|\hat{V}| \Psi_{\mathbf{n}_{r}}^{+}(E)\right\rangle \\
& =\left\langle(\hat{H}-E) \Phi_{\mathbf{n}_{p}}(E)\left|\left[\hat{1}+\hat{G}^{+}(E)(\hat{H}-E)\right]\right| \Phi_{\mathbf{n}_{r}}(E)\right\rangle \\
& =\left\langle\chi_{\mathbf{n}_{p}}(E) \mid \Phi_{\mathbf{n}_{r}}(E)\right\rangle+\left\langle\chi_{\mathbf{n}_{p}}(E)\left|\hat{G}^{+}(E)\right| \chi_{\mathbf{n}_{r}}(E)\right\rangle .
\end{aligned}
$$

Thus, it is clear that to determine the reactive transition amplitude, one only needs to know the Green's function elements which couple the reactant and product interaction regions.

The total interaction region (TIR) is the portion of configuration space where a square integrable $\left(L^{2}\right)$ basis set would be used to expand $\hat{G}^{+}(E)$. The computational effort of such a calculation would depend very strongly on the size of the $L^{2}$ basis set required. If $\left|\Phi_{\mathbf{n}_{r}}(E)\right\rangle$ were "distorted" to contain the effects of long range interactions (i.e. elastic and inelastic scattering), the $L^{2}$ basis set used to represent the TIR where only reactive events take place would be much smaller than if $\left|\Phi_{\mathbf{n}_{r}}(E)\right\rangle$ were a completely undistorted wave. Inelastically distorted waves have been used in both time dependent $[10]$ and time independent $[4,11]$ reactive scattering calculations. Although inelastically distorted waves are not explicitly constructed in this dissertation, we nevertheless attempt to include the effect of any long range interaction in $\left|\Phi_{\mathbf{n}_{r}}(E)\right\rangle$. For example, in Chapter 5 we show how a certain angular momentum coupling scheme can reduce the TIR.

The third point, which is a corollary of the second, is pursued in the ABC formulation. Namely, the form of $\hat{G}^{+}(E)$ in the region where $\chi_{\mathbf{n}_{r}}\left(R_{r}, E\right) \cong 0$, i.e. the asymptotic region, does not affect the reaction probabilities. Therefore, we are free to alter the nature of the Green's function in the asymptotic region, as long as the TIR elements are correct. This point is fully exploited in the next Section, in discussing the use of absorbing boundary conditions.

The final modification to the integral equation is not absolutely crucial, but rather is a matter of convenience. Defining the pre-limit Green's function by

$$
\hat{G}^{\epsilon}(E) \equiv(E+i \epsilon-\hat{H})^{-1}
$$


we can write Eq. (4.11) as

$$
\begin{aligned}
\left|\Psi_{\mathbf{n}_{r}}^{+}(E)\right\rangle & =\lim _{\epsilon \rightarrow 0^{+}}\left[\hat{1}+\hat{G}^{\epsilon}(E)(\hat{H}-E)\right]\left|\Phi_{\mathbf{n}_{r}}(E)\right\rangle \\
& =\lim _{\epsilon \rightarrow 0^{+}} \hat{G}^{\epsilon}(E)[(E+i \epsilon-\hat{H})+(\hat{H}-E)]\left|\Phi_{\mathbf{n}_{r}}(E)\right\rangle \\
& =\lim _{\epsilon \rightarrow 0^{+}} \hat{G}^{\epsilon}(E) i \epsilon\left|\Phi_{\mathbf{n}_{r}}(E)\right\rangle .
\end{aligned}
$$

This integral equation for the state ket is convenient for two reasons. The first point is somewhat trivial: it is easier to construct $i \epsilon\left|\Phi_{\mathbf{n}_{r}}(E)\right\rangle$ than it is to obtain $(\hat{H}-E)\left|\Phi_{\mathbf{n}_{r}}(E)\right\rangle$. The second reason, which is more important, will become clear after introducing the absorbing boundary conditions.

Much of the formal analysis given above relies on defining the Green's function in terms of the Born expansion. As such, these arguments seem to depend on the convergence of the Born expansion. However, the state kets in Eqs. (4.4), (4.9), (4.11), and (4.14) formally solve the Schrödinger equation provided that the following Green's function relations hold:

$$
\begin{aligned}
\hat{1} & =(E-\hat{K}) \hat{G}_{0}^{+}(E) \\
& =(E-\hat{H}) \hat{G}^{+}(E) \\
& =(E+i \epsilon-\hat{H}) \hat{G}^{\epsilon}(E) .
\end{aligned}
$$

That is, the integral equations derived above are well defined regardless of the convergence properties of the Born expansion.

Thus far in this Chapter, we have shown how to obtain the IRP directly from the scattering wavefunction, for which a convenient integral equation was constructed. We have emphasized that only the TIR portion of the Green's function contributes directly to the reaction probability. However, we have not yet shown how to determine the Green's function. In principle, the TIR portion of $\hat{G}^{\epsilon}(E)$ is correct only if $\epsilon$ is made small enough, i.e. only if the boundary conditions are properly enforced. As $\epsilon \rightarrow 0^{+}, \hat{G}^{\epsilon}(E)$ develops outgoing waves in all open channels extending over an infinite region of asymptotic space. Thus, the reaction probabilities is correct only if the asymptotic region is sampled in some fashion in determining $\hat{G}^{+}(E)$. In previous $L^{2}$ variational Green's function calculations [12], the outgoing waves have been represented by including infinitely delocalized functions in the basis set. Unfortunately, this gives rise to an awkward structure in the resulting Hamiltonian matrix. 
For technical reasons discussed below, this structure makes it very difficult to perform IRP calculations on large chemical systems. To solve this problem, we outline the $A B C$ version $[13,4]$ of the Green's function in Eq. (4.13) and the integral equation in Eq. (4.14), which facilitates IRP calculations on large chemical systems.

\subsubsection{Absorbing Boundary Conditions}

The basic principle behind the $A B C$ formulation is that accurate reaction probabilities can be determined while only sampling a finite region of space. This is achieved by replacing the infinitesimal energy $\epsilon$ in Eqs. (4.13) and (4.14) with a finite, coordinate dependent function $\epsilon(\mathbf{q}) \geq 0$. This modification gives the $A B C$ analogues of Eqs. (4.13) and (4.14),

$$
\hat{G}^{A B C}(E) \equiv(E+i \hat{\epsilon}-\hat{H})^{-1}
$$

and

$$
\left|\Psi_{\mathbf{n}_{r}}^{A B C}(E)\right\rangle=\hat{G}^{A B C}(E) i \hat{\epsilon}\left|\Phi_{\mathbf{n}_{r}}(E)\right\rangle .
$$

The kernel of $\hat{G}^{A B C}(E)$ is an $L^{2}$ function which decays exponentially where $\epsilon(\mathbf{q})$ is non-zero. In what follows, we omit the "ABC" superscript with the understanding that we are using the $\mathrm{ABC}$ formulation.

To obtain accurate reaction probabilities in an efficient manner with the $A B C$ formulation, the absorbing potential $\epsilon(q)$ must satisfy three criteria. The first and second pertain to accuracy, and the third to efficiency. First, $\epsilon(\mathbf{q})$ should be negligible in the TIR, where the Green's function directly contributes to the reaction probabilities. Second, the absorbing potential should not be so strong that it reflects amplitude back into the interaction region, effectively contaminating the outgoing wave character with incoming waves. Third, the absorbing potential should be strong enough to absorb the ABC Green's function as fast (in space) as possible, thereby reducing the $L^{2}$ basis set size required to represent it. The optimal absorbing potential, determined by the competition between accuracy and efficiency, is found through empirical testing. 
As an aside, we note that since $\epsilon(\mathbf{q}) \cong 0$ in the strong chemical interaction region, the product $\hat{\epsilon}\left|\Phi_{\mathbf{n}_{r}}(E)\right\rangle$ projects onto the asymptotic portion of $\left|\Phi_{\mathbf{n}_{r}}(E)\right\rangle$ only. Thus, it is not necessary to explicitly regularize $\left|\Phi_{\mathbf{n}_{r}}(E)\right\rangle$ at the origin. This is the second convenient aspect of the integral equation in Eqs. (4.14) and (4.17), as discussed in the previous Section.

We now turn our attention to the calculation of averaged reaction probabilities (e.g. the IRP) with the ABC formulation. This is perhaps its most important application, because one can determine averaged reaction probabilities with only partial information about the scattering wavefunction. In particular, since the IRP contains only initial state selection, the TIR which determines this quantity is larger on the reactant side than it is on the product side. That is because state selection is determined by inelastic scattering, which is usually a longer ranged interaction than is reactive scattering. The absorbing potential in all product chemical arrangements can be brought very close to the chemical exchange region, withoug losing accuracy in the IRP. Consequently, the size of the $L^{2}$ basis on the product side is greatly reduced by calculating the IRP. Thus, the ABC formulation facilitates more economical calculations for more averaged reaction probabilities.

The ABC analog of the IRP in Eq. (4.2) is obtained by representing the reactive flux operator $\hat{F}$ as the following commutator $[13,14]$,

$$
\hat{F}=\frac{1}{i \hbar}[\hat{h}(f), \hat{H}-E]
$$

where $h(x)$ is the step function, i.e. $h(x)=1$ for $x>0$ and $h(x)=0$ otherwise. Also, $f$ is some function which, via the equation $f=0$, defines a dividing surface between reactants and products. Using the fact that [cf. Eq. (4.16)]

$$
(\hat{H}-E) \hat{G}(E)=-\hat{1}+i \hat{\epsilon} \hat{G}(E)
$$

the IRP becomes:

$$
\begin{array}{r}
P_{\mathbf{n}_{r}}(E)=\frac{2}{\hbar}\left\langle\Phi_{\mathbf{n}_{r}}(E)\left|\hat{\epsilon} \hat{G}^{\dagger}(E) \hat{\epsilon}_{p} \hat{G}(E) \hat{\epsilon}\right| \Phi_{\mathbf{n}_{r}}(E)\right\rangle+ \\
\frac{2}{\hbar}\left\langle\Phi_{\mathbf{n}_{r}}(E)\left|\hat{\epsilon}_{p} \operatorname{Im} \hat{G}(E) \hat{\epsilon}\right| \Phi_{\mathbf{n}_{r}}(E)\right\rangle .
\end{array}
$$


In Eq. (4.19), $\hat{\epsilon}_{p}=\hat{\epsilon} \hat{h}(f)$ is the absorbing potential in the product region of configuration space. In practice, the magnitude of the vector $\hat{\epsilon}_{p}\left|\Phi_{\mathbf{n}_{r}}(E)\right\rangle$ is vanishingly small. [An exception to this occurs when $\left|\Phi_{\mathbf{n}_{r}}(E)\right\rangle$ is extremely vibrationally excited. We do not consider such initial conditions.] As such, the second term in Eq. (4.19) is negligible, and the final form for the $A B C$ version of the IRP is given by

$$
P_{\mathbf{n}_{r}}(E)=\frac{2}{\hbar}\left\langle\Psi_{\mathbf{n}_{r}}(E)\left|\hat{\epsilon}_{p}\right| \Psi_{\mathbf{n}_{r}}(E)\right\rangle
$$

where $\left|\Psi_{\mathbf{n}_{r}}(E)\right\rangle$ is given by Eq. (4.17). In the ABC formula for the IRP, the operator $2 \hat{\epsilon}_{p} / \hbar$ has replaced the reactive flux operator $\hat{F}$, and the $A B C$ wavefunction has replaced the exact one. Equation (4.20) is the working formula for the reactive scattering calculations reported in the remainder of this dissertation.

To complete the discussion of the $\mathrm{ABC}$ formulation, we make two general comments. First, the formal Green's function involves an infinitesimal "absorbing potential" $\epsilon \rightarrow 0^{+}$over an infinite region of space. The ABC Green's function, on the other hand, is defined by a finite absorbing potential over a finite region of space. With some care (and some convergence tests), the reaction probabilities obtained from both should be identical. Second, all methods which obtain the Green's function must simulate the outgoing wave boundary conditions. Previous $L^{2}$ variational methods enforce the boundary conditions directly in the basis set, giving the problems mentioned above. The ABC formulation, on the other hand, effectively enforces the boundary conditions in the Hamiltonian. Although this latter procedure is somewhat more ad hoc than the former, it has a distinct advantage which we exploit in the next Section.

\subsection{Polynomial Expansions}

A finite basis representation of Eq. (4.17) gives

$$
\boldsymbol{\Psi}_{\mathbf{n}_{r}}(E)=\mathbf{G}(E) i \boldsymbol{\epsilon} \boldsymbol{\Phi}_{\mathbf{n}_{r}}(E)
$$

which is the solution to the non-Hermitian linear system of simultaneous equations:

$$
(E+i \epsilon-\mathbf{H}) \boldsymbol{\Psi}_{\mathbf{n}_{r}}(E)=i \epsilon \boldsymbol{\Phi}_{\mathbf{n}_{r}}(E) .
$$


As the strength of $\epsilon$ goes to zero and the spatial extent of the basis becomes complete, Eq. (4.22) approaches the time independent Schrödinger equation with scattering boundary conditions in Eq. (4.3). As such, appropriate convergence tests are required to ensure that $\epsilon$ is not too strong and that the basis samples a sufficiently large region of configuration space. Figure 4.1 illustrates, for a simple collinear reaction system, the space required to obtain a typical IRP accurately. We notice that the grid size on the product side (upper left) where no state selection is obtained, is smaller than that on the reactant side (lower right). In addition, the absorbing potential on the reactant side must be more gentle than that on the product side. This demonstrates the computational economy of directly calculating the IRP.

The $\mathrm{ABC}$ method reduces the quantum reactive scattering problem to solving the linear system in Eq. (4.22). The most straightforward method of solution is LU decomposition [15], which requires storage of the Hamiltonian matrix. In LU decomposition, the system matrix is initially factorized into the product of a lower triangular and an upper triangular matrix, requiring $N^{3} / 3$ operations where $N$ is the system size. If solution vectors are computed for a small number of right hand side vectors (i.e. initial reactant states $\mathbf{n}_{\mathbf{r}}$ ), this factorization is the most time consuming step. Given the specific right hand side vector and the results of the factorization, the solution vector is obtained rapidly with back substitution. This is the method of choice for small systems (i.e. one or two dimensions), or for larger systems in which $L^{2}$ basis set contraction schemes allow the Hamiltonian matrix to be stored. These contraction schemes are somewhat system dependent, and have only been tested on three dimensional scattering systems $[16,17]$. A more general approach, however, for performing scattering calculations on large systems is the use of a grid representation for the Hamiltonian in conjunction with an iterative solution of the resulting sparse linear system. This is because iterative methods do no require storage of the Hamiltonian and are especially rapid when used to solve sparse systems.

The power of a grid representation derives from the simplicity of the kinetic energy - usually a sum of one dimensional terms. As such, a basis representation which is diagonal in coordinate space gives a simple form for the Hamiltonian matrix. In particular, constructing the Hamiltonian is simple with a grid because no multi- 


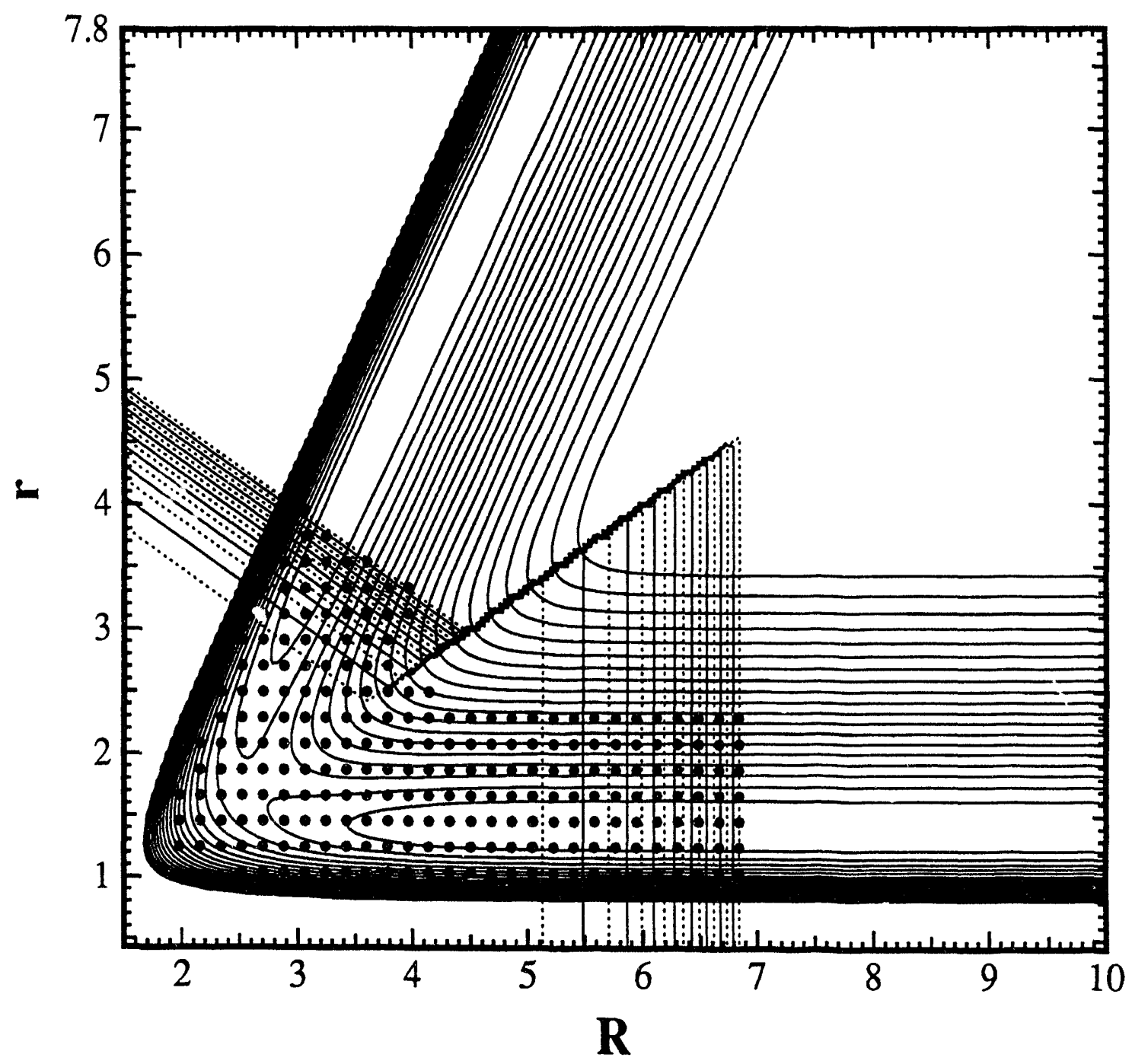

Figure 4.1: Configuration space required to determine the IRP accurately, for collinear $\mathrm{H}+\mathrm{H}_{2}(v=1)$ at $E=0.99 \mathrm{eV}$. The solid contours denote the potential energy surface, the dashed contours show the absorbing boundary regions, and the dots are the centers of basis functions used for the calculation. The jagged line between the two absorbing potentials is the dividing line between reactants (below) and products (above). We note that the grid is much smaller on the product side, where there is no state selection, than on the reactant side. Also, the absorbing potential can be much stronger on the product side. This demonstrates the economy of directly calculating the IRP. 
dimensional quadratures over the potential function are required. Also, the resulting matrix is sparse because the potential is diagonal. To appreciate the sparsity, we consider a two dimensional system with rectilinear coordinates $(x, y)$. The coupling is contained in the kinetic energy - we consider the $x$ dependent term. Labeling the $x$ grid with $\left(i, i^{\prime}\right)$ and the $y$ grid with $\left(j, j^{\prime}\right)$, the $\left(i j, i^{\prime} j^{\prime}\right)$ element of $\hat{T}^{x}$ is

$$
\begin{aligned}
\left\langle i j\left|\hat{T}^{x}\right| i^{\prime} j^{\prime}\right\rangle & =\left\langle i j\left|\hat{t}^{x} \otimes \hat{1}^{y}\right| i^{\prime} j^{\prime}\right\rangle \\
& =\left\langle i\left|\hat{t}^{x}\right| i^{\prime}\right\rangle \delta_{j, j^{\prime}} .
\end{aligned}
$$

Since in most cases the factor $\delta_{j, j^{\prime}}=0$ and the potential is diagonal, the Hamiltonian is sparse. We suppose now that an $F$ dimensional system has $n$ grid points per degree of freedom, yielding a total grid size $N=n^{F}$. To multiply the grid Hamiltonian into an arbitrary vector requires $(n F+1) N$ multiplications, as opposed to $N^{2}$ for a full matrix. On the down side, any localized basis set has large kinetic energy components. As discussed below, the time required by most iterative methods is roughly proportional to the spectral range of the system. An important area of active research is, thus, the development of grid methods which retain sparsity while reducing the spectral range [18].

The two most frequently used grid methods to solve the Schrödinger equation are the discrete variable representation [19-21] (DVR), and the fast Fourier transform method [6, 22] (FFT). [Please see Chapter 3 for a critical comparison between the two grid methods.] In this dissertation, we exclusively use DVR because it allows us to tailor the grid, in a simple fashion, to the shape of the physical and absorbing potentials.

A method of solving linear systems is usually called "iterative" if it involves several steps (i.e. iterations) of a matrix multiplying a vector. [We note that all numerical wavepacket propagation schemes fall into this class (see also Chapter 3).] Most iterative methods involve applying a linear function of the Hamiltonian matrix, thereby forming the solution vector as an expansion in powers of the Hamiltonian. The number of operations required by such an iterative method would be $N_{i t e r}(n F+1) N$, where $N_{i t e r}$ is the number of iterations required for convergence. For the reasons stated in the Introduction, namely numerical convenience and effi- 
ciency, we ask the question: Can the $A B C$ wavefunction be reliably obtained with such a polynomial expansion? Several possible pulynomial based iterative schemes are analyzed to answer this question.

\subsubsection{Direct Expansion}

We define a direct polynomial expansion of the ABC Green's function (and equivalently the $\mathrm{ABC}$ wavefunction) by anything having the form:

$$
\mathrm{G}(E) \cong \sum_{n=0}^{N} c_{n}(E) P_{n}(\mathbf{H}-i \boldsymbol{\epsilon}),
$$

where $P_{n}(z)$ is a polynomial in $z$, and $c_{n}$ is an energy dependent coefficient.

\section{The Distorted Born Expansion}

The distorted Born expansion [3] (DBE) is a simple example of a polynomial expansion for the scattering Green's function. The form is not strictly that given in Eq. (4.24), but is close enough to be seriously considered. Defining $\mathbf{H}_{0}$ as a reference system (whose ABC Green's function is easy to construct), and $\mathbf{H}_{\mathbf{1}}=\mathbf{H}-\mathbf{H}_{0}$ as the perturbation, the $\mathrm{DBE}$ is

$$
\mathbf{G}(E)=\mathbf{G}_{0}(E) \sum_{n=0}^{\infty}\left[\mathbf{H}_{1} \mathbf{G}_{0}(E)\right]^{n}
$$

where

$$
\mathrm{G}_{0}(E)=\left(E+i \epsilon-\mathbf{H}_{0}\right)^{-1} \text {. }
$$

This converges only if the eigenvalues of $\left[\mathbf{H}_{1} \mathbf{G}_{0}(E)\right]$ all have modulus less than one. Clearly, in the limit that $\mathbf{H}_{1}$ is small (and $\mathbf{G}_{0}(E)$ approaches the full ABC Green's function), the convergence of the DBE is guaranteed. Thus, a sufficiently good reference Green's function is required for the DBE to be useful. For this reason, the $\mathrm{DBE}$ has been used mostly has a means of obtaining first order corrections to the dynamics of reasonable reference systems, i.e. the distorted wave Born approximation (DWBA). An important example of a sophisticated implementation of the DWBA is the $S$-matrix version of the Kohn variational principle [23]. 
For small systems, the matrix $\left[\mathrm{H}_{1} \mathrm{G}_{0}(E)\right]$ in the $\mathrm{ABC}$ formulation can be diagonalized and its eigenvalues inspected to test for convergence of the DBE. We show the results of such an exercise for two different systems. The first, in Fig. 4.2 , is for collinear $\mathrm{H}+\mathrm{H}_{2}$ at total energy $E=0.99 \mathrm{eV}$, using a two dimensional grid basis. In this case, $\mathbf{H}_{1}$ is the off-diagonal portion of the Hamiltonian matrix, and $\mathbf{G}_{0}(E)$ is the $\mathrm{ABC}$ Green's Iunction for the remaining diagonal part. In Fig. 4.2 , the interior of the unit circle defines the convergence regime. Since there are eigenvalues of $\left.{ }_{[} \mathbf{H}_{1} \mathbf{G}_{0}(E)\right]$ with modulus greater than one, the DBE would diverge. A more sophisticated reference system is required. The second system considered is the three dimensional $\mathrm{H}+\mathrm{O}_{2}$ reaction at $E=3.0 \mathrm{eV}$, represented in an adiabatically contracted basis set [18]. In this latter case, $\mathrm{G}_{0}(E)$ is the inverse of the portion of the Hamiltonian which is diagonalized in the contraction, and $\mathbf{H}_{1}$ the remainder. Figure 4.3 shows that even with this more sophisticated reference system, the DBE would diverge. Thus, we seek a more generaily reliable alternative.

\section{The Krylov Space}

Two popular methods for solving the $N \times N$ linear system $\mathbf{A x}=\mathbf{b}$ are based on constructing the Krylov representation of $\mathbf{A}[24,25]$. The Krylov space of dimension $p$ is defined as the span of the set of vectors

$$
\left\{\mathbf{b}, \mathbf{A} \mathbf{b}, \mathbf{A}^{2} \mathbf{b}, \cdots, \mathbf{A}^{p-1} \mathbf{b}\right\}
$$

where $p \leq N$ and usually $p \ll N$. We can define an orthonormal basis $\mathbf{V}_{p}$ for the Krylov space spanned by $(4.27)$, with the properties

$$
\mathbf{V}_{p}=\left\{\mathbf{v}_{0}, \mathbf{v}_{1}, \mathbf{v}_{2}, \cdots, \mathbf{v}_{p-1}\right\}
$$

and

$$
\left(\mathbf{v}_{i}, \mathbf{v}_{j}\right) \equiv \mathbf{v}_{i}^{\dagger} \cdot \mathbf{v}_{j}=\delta_{i j}
$$

This basis can be generated using Gram-Schmidt orthogonalization, yielding the recursion relations:

$$
\begin{aligned}
& \beta_{0} \mathbf{v}_{0}=\mathbf{b} \\
& \beta_{i+1} \mathbf{v}_{i+1}=\mathbf{A} \mathbf{v}_{i}-\sum_{j=0}^{i}\left(\mathbf{v}_{j}, \mathbf{A} \mathbf{v}_{i}\right) \mathbf{v}_{j}, \quad \text { for } i=0, \ldots, p-2
\end{aligned}
$$




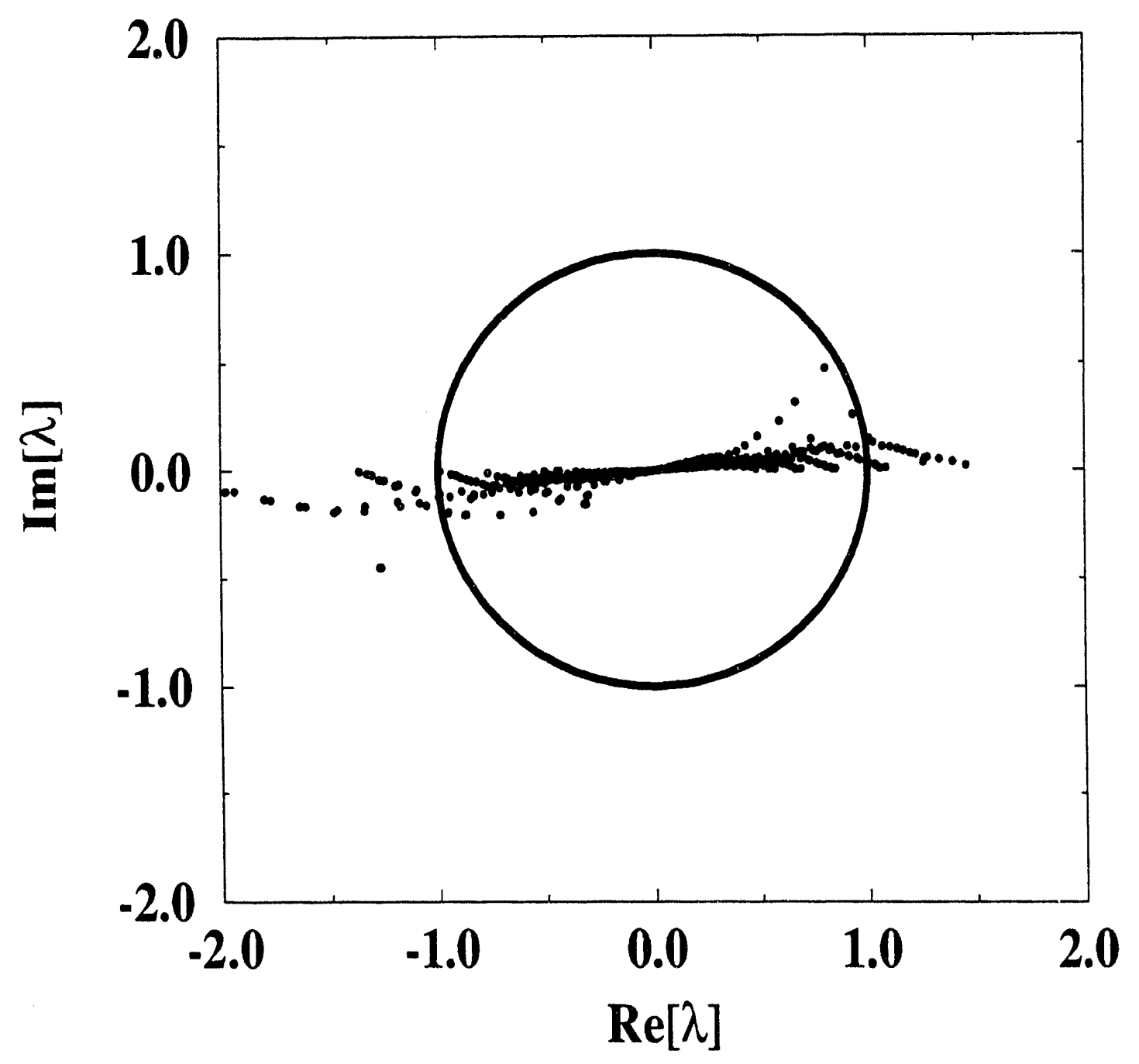

Figure 4.2: The eigenvalue spectrum of $\left[\mathbf{H}_{1} \mathbf{G}_{0}(E)\right]$ for collinear $\mathrm{H}+\mathrm{H}_{2}$ at total energy $E=0.99 \mathrm{eV}$, using a two dimensional grid basis. In this case, $\mathbf{H}_{1}$ is the off-diagonal portion of the Hamiltonian matrix, and $\mathrm{G}_{0}(E)$ is the $A B C$ Green's function for the remaining diagonal part. Since there are eigenvalues of $\left[\mathrm{H}_{1} \mathrm{G}_{0}(E)\right]$ with modulus greater than one, the DBE would diverge. 


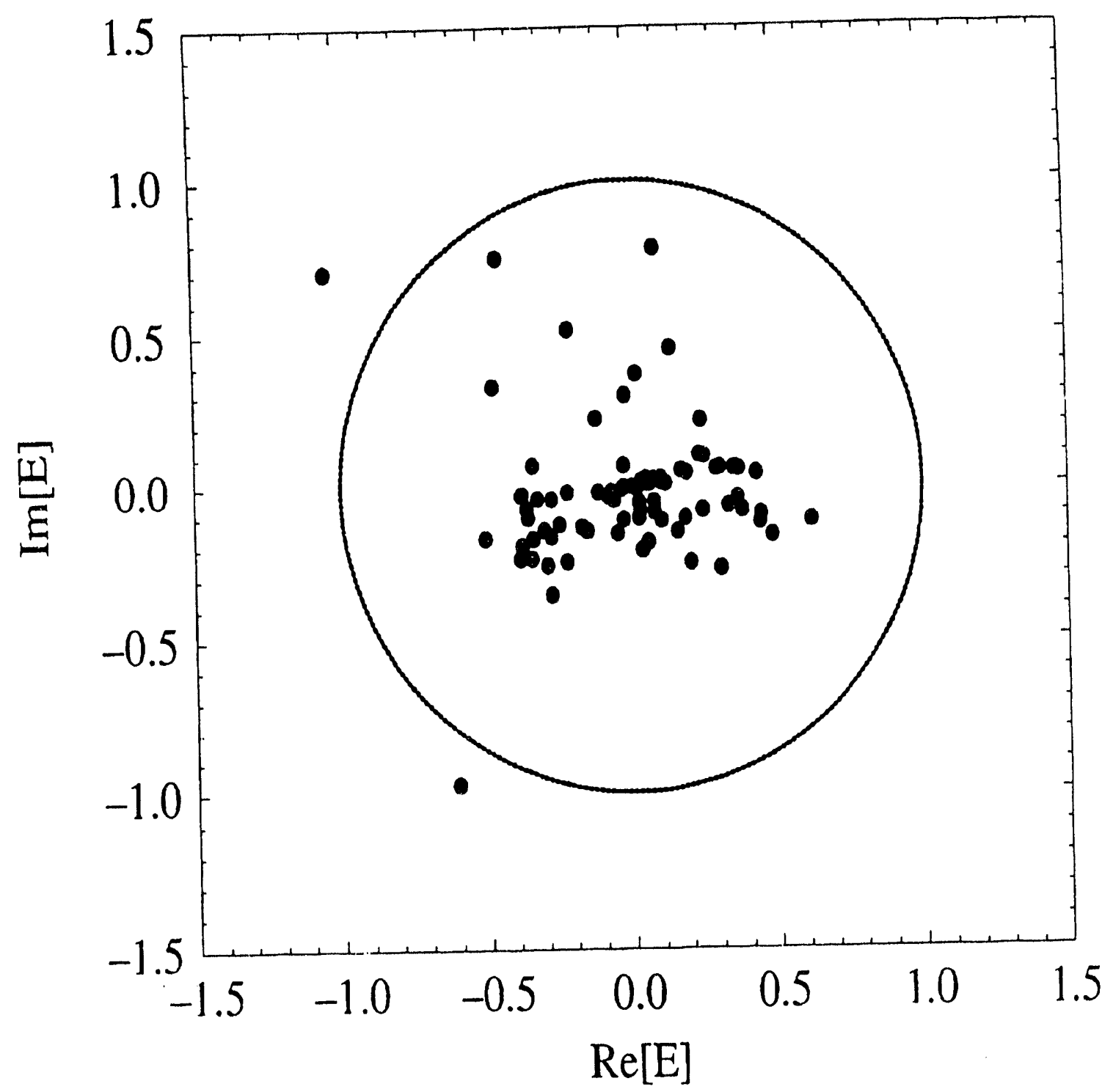

Figure 4.3: The eigenvalue spectrum of $\left[\mathbf{H}_{1} \mathbf{G}_{0}(E)\right]$ for three dimensional $\mathrm{H}+\mathrm{O}_{2}$ at total energy $E=3.0 \mathrm{eV}$, using a three dimensional grid basis. In this case, $\mathbf{G}_{0}(E)$ is the inverse of the portion of the Hamiltonian which is diagonalized in the contraction, and $\mathbf{H}_{1}$ the remainder. Since there are eigenvalues of $\left[\mathbf{H}_{1} \mathbf{G}_{0}(E)\right]$ with modulus greater than one, the DBE would diverge. 
where $\beta_{i}$ is chosen so that $\left\|\mathbf{v}_{i}\right\| \equiv \sqrt{\left(\mathbf{v}_{i}, \mathbf{v}_{i}\right)}=1$. The matrix $\mathbf{A}$ in the $\mathbf{v}_{p}$ basis is the $p \times p$ matrix $\tilde{\mathbf{A}}$ defined by:

$$
\tilde{\mathbf{A}}_{i j}=\left(\mathbf{V}_{p}^{\dagger} \mathbf{A} \mathbf{V}_{p}\right)_{i j}=\left(\mathbf{v}_{i}, \mathbf{A} \mathbf{v}_{j}\right)
$$

Since $\mathbf{A} \mathbf{v}_{j} \in \operatorname{span}\left(\mathbf{V}_{j+1}\right)$, and $\mathbf{v}_{i} \in \operatorname{span}\left(\mathbf{V}_{j+1}\right)$ only if $i \leq j+1, \tilde{\mathbf{A}}$ has the important property that $\tilde{\mathbf{A}}_{i j}=0$ for $i>j+1$. This kind of matrix, known as upper-Hessenberg, is usually much easier to manipulate than $\mathbf{A}$ because $\tilde{\mathbf{A}}$ is almost upper triangular. Thus, the philosophy behind the Krylov space based methods (KSM) is to transform the original linear system into the simpler form $\tilde{A} \tilde{\mathbf{x}}=\tilde{\mathbf{b}}$ where $\tilde{\mathbf{x}}=\mathbf{V}_{p}^{\dagger} \mathbf{x}$ and $\tilde{\mathbf{b}}=\mathbf{V}_{p}^{\dagger} \mathbf{b}$, which can be solved more easily. The computational effort is usually dominated by the construction and orthogonalization of the transformation matrix $V_{p}$, which requires a matrix vector multiply at each iteration.

The KSM are particularly useful in two cases. To discuss the first, we define the eigenvalues of $\mathbf{A}$ by $\left\{\lambda_{1}, \lambda_{2}, \ldots, \lambda_{N}\right\}$ such that $\left|\lambda_{1}\right| \leq\left|\lambda_{2}\right| \leq \ldots \leq\left|\lambda_{N}\right|$. The KSM converges rapidly when the condition number $\mathcal{C} \equiv\left|\lambda_{N}\right| /\left|\lambda_{1}\right|$ is small. That is because the KSM tend to span the eigenvectors of $\mathbf{A}$ from both ends of the spectrum first, filling in the intermediate spectral region only after several iterations have been performed. The second case in which the KSM are useful is when $\mathbf{A}$ is Hermitian. In that case, $\tilde{\mathbf{A}}$ is also Hermitian, and thus tridiagonal. The recursion relations in Eq. (4.30) simplify to the following three term recursion relation:

$$
\begin{aligned}
& \beta_{0} \mathbf{v}_{0}=\mathbf{b} \\
& \beta_{i+1} \mathbf{v}_{i+1}=\left(\mathbf{A}-\alpha_{i}\right) \mathbf{v}_{i}-\beta_{i}^{*} \mathbf{v}_{i-1}, \quad \text { for } i=0, \ldots, p-2
\end{aligned}
$$

where

$$
\begin{aligned}
\tilde{\mathbf{A}}_{i i}=\alpha_{i} & =\left(\mathbf{v}_{i}, \mathbf{A} \mathbf{v}_{i}\right) \\
\tilde{\mathbf{A}}_{i+1, i}=\beta_{i+1} & =\left(\mathbf{v}_{i+1}, \mathbf{A} \mathbf{v}_{i}\right) \\
& =\left\|\left(\mathbf{A}-\alpha_{i}\right) \mathbf{v}_{i}-\beta_{i}^{*} \mathbf{v}_{i-1}\right\| .
\end{aligned}
$$

This is known as the Lanczos recursion formula [26], first developed for computing eigenvalues of Hermitian matrices. The vectors so obtained are called the Lanczos vectors. The tridiagonal linear system can be solved almost trivially with the LQ 
method [15]. This is the basic approach behind the SYMMLQ algorithm of Paige and Saunders [27]. SYMMLQ only requires the storage of three Lanczos vectors, in addition to the $\left\{\alpha_{i}\right\}$ and $\left\{\beta_{i}\right\}$ values, and thus is applicable to the solution of large systems. It is used in the $S$-matrix Kohn variational scattering calculations of Groenenboom and Colbert [11].

The accuracy of the Lanczos recursion formula is notoriously sensitive to the finite precision arithmetic used in actual numerical calculations. This is because the Lanczos vectors which are not explicitly orthogonalized to eachother (e.g. $\mathbf{v}_{i+1}$ and $\mathbf{v}_{i-2}$ ) can become numerically linearly dependent. As such, altliough the solution vector must converge in no more than $N$ iterations in infinite precision, such convergence is not guaranteed for SYMMLQ if it is numerically implemented with the Lanczos recursion formula. In addition, although the two equations for $\beta_{i+1}$ in Eq. (4.33) are mathematically identical, the second is better behaved in finite precision. Lanczos based algorithms which stablilize the effect of round off error have been developed [28], which make them powerful methods for diagonalizing or solving sparse, Hermitian systems. Of course, the $\mathrm{ABC}$ formulation of quantum reactive scattering gives a non-Hermitian matrix $\mathbf{A}=(E+i \boldsymbol{\epsilon}-\mathbf{H})$. To use the KSM for the $\mathrm{ABC}$ system, there are three basic approaches one may take.

The simplest approach is to convert the non-Hermitian linear system in Eq. (4.22) into a Hermitian one by premultiplying both sides by $(E-i \epsilon-\mathbf{H})$, yielding the following linear system:

$$
\left\{(E-\mathbf{H})^{2}+\boldsymbol{\epsilon}^{2}+i[E-\mathbf{H}, \boldsymbol{\epsilon}]\right\} \boldsymbol{\Psi}_{\mathbf{n}_{r}}(E)=(E-i \boldsymbol{\epsilon}-\mathbf{H}) i \boldsymbol{\epsilon} \boldsymbol{\Phi}_{\mathbf{n}_{r}}(E)
$$

where $[\mathbf{A}, \mathbf{B}]=\mathbf{A B}-\mathbf{B A}$ is the commutator. One could then use the SYMMLQ algorithm to find the $\mathrm{ABC}$ wavefunction. Although this approach should work in principle, it is not advised because the condition number of the Hermitian system is roughly the square of that from the original system. That usually spells disaster for an iterative scheme, because the number of iterations required for convergence depends quite strongly on the condition number.

The second approach forces the Krylov representation of the ABC linear system to be complex symmeiric, and uses a somewhat inferior version of SYMMLQ 
to solve the resulting equation. Although $\mathbf{A}$ is complex symmetric with real basis functions (e.g. a grid representation), $\tilde{\mathbf{A}}$ is non-symmetric because the $\mathbf{V}_{p}$ basis is complex when computed for the $\mathrm{ABC}$ system. However, for any basis representation, using a biorthogonal inner product (BIP) forces $\tilde{\mathbf{A}}$ to be complex symmetric. The BIP uses the transpose rather than the Hermitian adjoint to compute the inner product, i.e.

$$
(\mathbf{u}, \mathbf{v})_{B I P} \equiv \mathbf{u}^{T} \cdot \mathbf{v}
$$

We note that the BIP is the inner product for which the eigenvectors of a complex symmetric matrix are orthogonal, which is the mathematical grounding for its use. With this modification, the Lanczos recursion formula becomes

$$
\begin{aligned}
& \beta_{0} \mathbf{v}_{0}=\mathbf{b} \\
& \beta_{i+1} \mathbf{v}_{i+1}=\left(\mathbf{A}-\alpha_{i}\right) \mathbf{v}_{i}-\beta_{i} \mathbf{v}_{i-1}, \quad \text { for } i=0, \ldots, p-2
\end{aligned}
$$

where

$$
\begin{aligned}
\tilde{\mathbf{A}}_{i i}=\alpha_{i} & =\left(\mathbf{v}_{i}, \mathbf{A} \mathbf{v}_{i}\right)_{B I P} \\
\tilde{\mathbf{A}}_{i+1, i}=\beta_{i+1} & =\left(\mathbf{v}_{i+1}, \mathbf{A} \mathbf{v}_{i}\right)_{B I P} \\
& =\left\|\left(\mathbf{A}-\alpha_{i}\right) \mathbf{v}_{i}-\beta_{i} \mathbf{v}_{i-1}\right\|_{B I P}
\end{aligned}
$$

Now $\tilde{\mathbf{A}}$ is complex symmetric, and the $\left\{\alpha_{i}\right\}$ and $\left\{\beta_{i}\right\}$ values are in general complex. Unfortunately, since a vector space with this inner product does not have a meaningful norm, it can be difficult to gauge the convergence of the approximate solution vector to the actual one. In fact, the convergence properties of the complex symmetric Lanczos algorithm are known to be much worse than the Hermitian one [29, 30]. Presumably, this is because the instabilities due to numerical round off error are greatly exacerbated using the BIP. In addition, it is much more difficult to stabilize the complex symmetric Lanczos algorithm than it is the Hermitian one [31]. For these reasons, we sought a more reliable alternative.

To avoid the difficulties outlined above in applying the KSM to the ABC problem, it is clear that one should implement the KSM as originally outlined in Eqs. (4.29)-(4.31). That is, one simply uses the usual inner product for which a 
meaningful norm exists, obtaining a non-symmetric upper-Hessenberg form for $\tilde{\mathbf{A}}$. The Gram-Schmidt orthogonalization procedure is no longer a three term recursion relation, but rather requires explicit orthogonaliztion to al' the previously computed Lanczos vectors. This is the basic approach of the generalized minimum residual method (GMRES) of Saad and Schultz [32], which obtains its added generality and stability at the expense of having to store all the computed Lanczos vectors. We wish to make three general comments about its use. First, since explicit orthogonaliztion is performed, GMRES is guaranteed to converge in no more than $N$ iterations. If this is required, LU decomposition is again the method of choice since storing $N$ Lanczos vectors is tantamount to storing the Hamiltonian matrix, and $\mathbf{L U}$ decomposition can give solution vectors for all right hand sides at total energy $E$. GMRES is the method of choice, however, when the Hamiltonian matrix can not be stored, while the Lanczos vectors required for convergence can be (i.e. many fewer than $N$ iterations are required). Therefore, we believe that GMRES is the first iterative method to try when LU decomposition is not possible.

The second remark concerns the numerical procedure known as preconditioning. This is a general name given to reducing the number of iterations required to solve a linear system. [Theoretical chemists think of "preconditioning" in the broader sense of choosing a better reference system, i.e. choosing coordinates, basis functions, or asymptotic states which better describe and decouple the system dynamics. All these would change the size of the linear system to be solved. Therefore, although this broad sense of preconditioning is clearly quite important, we restrict ourselves to the more narrow notion of preconditioning to be discussed below.] We suppose that an $N \times N$ matrix $\mathbf{P}$, which is close to $\mathbf{A}$ in some sense, is known and easily invertible. Then the preconditioned linear system is given by $\mathbf{P}^{-1} \mathbf{A x}=\mathbf{P}^{-1} \mathbf{b}$. Of course, in the limit that $\mathbf{P} \rightarrow \mathbf{A}$, the preconditioned system becomes $\mathbf{1 x}=\mathbf{A}^{-1} \mathbf{b}$, and the system is solved. That this limit is not practically attainable is the subject of this Chapter. A preconditioner intermediate between 1 (i.e. no preconditioning) and $\mathbf{A}$ (i.e. complete preconditioning) can, however, be quite useful.

Preconditioning can affect the linear system in two somewhat distinct ways. 
First, it makes the system more diagonally dominant, i.e. the spectral dispersion is reduced. In the limit of complete diagonal dominance, the linear system is diagonal and solution is trivial. Second, preconditioning reduces the spectral range of the system. In the limit of complete preconditioning, the new system has $N$ eigenvalues equal to unity, and the spectral range is zero. Consequently, the condition number $\mathcal{C} \equiv\left|\lambda_{N}\right| /\left|\lambda_{1}\right|=1$ and solution is again trivial. One may think of preconditioning the $A B C$ linear system as similar to choosing a better reference Green's function in the distorted Born expansion. Alternatively, in the time domain, preconditioning is tantamount to choosing a better reference system in the Interaction Picture [33].

Preconditioning is especially relevant to GMRES because it both allows and requires preconditioning. The SYMMLQ algorithm can only be successfully preconditioned when used to solve a positive definite system. That is because the preconditioner must be applied symmetrically via

$$
\left[\mathbf{P}^{-1 / 2} \mathbf{A P}^{-1 / 2}\right] \mathbf{P}^{+1 / 2} \mathbf{x}=\mathbf{P}^{-1 / 2} \mathbf{b}
$$

The resulting system $\left[\mathbf{P}^{-1 / 2} \mathbf{A} \mathbf{P}^{-1 / 2}\right]$ is Hermitian only if $\mathbf{A}$ is Hermitian and $\mathbf{P}$ is positive definite. But $\mathbf{P}$ is close to $\mathbf{A}$ only if $\mathbf{A}$ is also positive definite. However, GMRES is able to handle arbitrary systems with arbitrary preconditioners. This fact, coupled with the storage requirements of GMRES, make preconditioning an important component of any attempt to use GMRES to solve large systems.

In all preconditioning schemes, it is important that the system $\mathbf{P y}=\mathbf{c}$ be relatively easy to solve, because such a system is solved at each GMRES iteration. Two particularly simple preconditioners are $\mathbf{P}=\operatorname{diag} \mathbf{A}$ and $\mathbf{P}=$ tridiag $\mathbf{A}$, both of which only require $O(N)$ operations to solve Py $=\mathbf{c}$. The tridiagonal preconditioner has demonstrated extremely impressive results for one dimensional elastic scattering systems [34]. That is presumably because the tridiagonal preconditioner is tantamount to using a three point finite difference formula for the second derivative kinetic energy coupling. Unfortunately, such an approximation to the kinetic energy for a multidimensional system gives rise to a matrix which is more complicated than tridiagonal. Accordingly, the tridiagonal preconditioner in multidimensional reactive systems has not given such impressive results [34]. Manolopoulos et al. have devel- 
oped a powerful preconditioner for performing inelastic atom surface scattering calculations with the log derivative version of the Kohn variational principle [35]. They construct a one dimensional average potential, and use multiple copies of this reference system for the various internal states that comprise the total $L^{2}$ basis, yielding a block diagonal preconditioner. Manolopoulos et al. were able to converge the solution vector to eight digit accuracy after 23 preconditioned GMRES iterations, whereas 50 unpreconditioned GMRES iterations gave only one digit accuracy. Unfortunately, no such dramatic results have been reported for reactive scattering systems. In fact, the many arrangement nature of a reactive system may make successful preconditioning very difficult, because there are several different reference (i.e. asymptotic) systems. Nevertheless, this is an important and active field of research [36].

When LU decomposition is not possible, and preconditioned GMRES runs out of memory before converging, what alternatives remain for solving the $A B C$ system? One may "restart" GMRES, in which the solution vector is constructed from the available Lanczos vectors. Denoting this unconverged solution as $\mathbf{x}_{0}$, one then defines the exact solution as $\mathbf{x} \equiv \mathbf{x}_{0}+\delta \mathbf{x}$, and solves for $\delta \mathbf{x}$ via $\mathbf{A} \delta \mathbf{x}=\mathbf{b}-\mathbf{A} \mathbf{x}_{0}$. If memory runs out again, the approximate solution vector is updated $\mathbf{x}_{0} \rightarrow \mathbf{x}_{0}+\delta \mathbf{x}$, and the procedure repeated. This iterative refinement may converge if $x_{0}$ is in the neighborhood of $\mathbf{x}$, i.e. if the number of Lanczos vectors that can be stored is not too far from the total number required. Thus, the question remains: Does any algorithm exist which is accurate, has a fixed and small memory requirement, and is guaranteed to converge. To pursue such a method, we consider next the Newton polynomial expansion.

\section{Newton Polynomial Expansion}

We may be able to make some progress in solving the ABC system by appealing to the Newton polynomial expansion, which is a useful method for approximating scalar functions. A special case is the Chebyshev polynomial expansion, which is widely used in commercial algorithms for evaluating special functions [37,38]. Based on the Chebyshev expansion for $u(x)=e^{-i x t}$ for $x$ real, Tal-Ezer and Kosloff [5] 
have developed a very powerful method for solving the time dependent Schrödinger equation by expanding the unitary propagator in Chebyshev polynomials. We seek an analogous method for the ABC Green's function by defining the non-Hermitian matrix $\mathbf{Z} \equiv \mathbf{H}-i \boldsymbol{\epsilon}$. The ABC Green's function is then $\mathbf{g}(\mathbf{Z})=(E-\mathbf{Z})^{-1}$, a function of the complex (matrix) variable $\mathbf{Z}$. The central idea behind the work of Tal-Ezer and Kosloff is that the convergence properties of the matrix expansion for $\mathbf{g}(\mathbf{Z})$ can be understood in terms of the scalar expansion for $g(z)$, as long as $z$ is in the eigenvalue spectrum of $Z$. Therefore, we examine the convergence properties of the scalar function $g(z)=(E-z)^{-1}$.

The Newton polynomial expansion arises from the theory of interpolation in the complex plane $[39,40]$. Its mathematical background is beautifully discussed by Berman et al. in Ref. 40. It is more numerically stable than a Taylor expansion because the interpolation based expansion is referenced to several points, while the Taylor expansion is referenced to a single point. Among the various formulations of polynomial interpolation, the Newtonian formulation is most suited to the development of iterative methods with small memory requirements. That is because the Newton expansion builds the higher degree terms from the lower ones. This allows the successively higher powers to be accumulated recursively, thereby requiring a minimal amount of core memory. In other interpolation schemes, e.g. the Lagrangian formulation, this is not possible.

To implement the Newton polynomial expansion, we first suppose a function $f$ is analytic on a compact domain $D$. Furthermore, defining the boundary of $D$ as $\Gamma, f$ is evaluated at complex sampling points $\left\{z_{k}\right\}$ on $\Gamma$ giving the set of interpolation support points $\left\{\left(z_{k}, f_{k}\right)\right\}$, where $f_{k}=f\left(z_{k}\right)$. An approximate representation of $f$ on $D$ is given by

$$
f(z) \cong P_{K}(z)
$$

where

$$
P_{K}(z) \equiv \sum_{k=0}^{K} a_{k} R_{k}(z)
$$


In Eq. (4.39), $R_{k}(z)$ is the Newton polynomial of degree $k$ defined by

$$
R_{k}(z) \equiv\left\{\begin{array}{ll}
1 & k=0 \\
\prod_{j=0}^{k-1}\left(z-z_{j}\right) & k>0
\end{array},\right.
$$

and $a_{k}$ is the $k^{\text {th }}$ divided difference coefficient [41], determined by requiring that $P_{k}\left(z_{k}\right)=f_{k}$ for each $k$. For example, considering $k=0$ :

$$
f_{0}=P_{0}\left(z_{0}\right)=a_{0} R_{0}\left(z_{0}\right)=a_{0} .
$$

Evaluating for $k=1$ gives:

$$
\begin{aligned}
f_{1}=P_{1}\left(z_{1}\right) & =a_{0} R_{0}\left(z_{1}\right)+a_{1} R_{1}\left(z_{1}\right) \\
& =a_{0}+a_{1}\left(z_{1}-z_{0}\right) \\
& =f_{0}+a_{1}\left(z_{1}-z_{0}\right) .
\end{aligned}
$$

As such, the coefficients $\left\{a_{k}\right\}$ are built up iteratively, in a way which can be summarized by

$$
a_{0}=f_{0}, a_{1}=\frac{f_{1}-f_{0}}{z_{1}-z_{0}}, \ldots, a_{k}=\frac{f_{k}-P_{k-1}\left(z_{k}\right)}{R_{k}\left(z_{k}\right)} .
$$

It is clear from Eq. (4.43) why the Newton expansion coefficients are called divided differences. It is also apparent from the definition of the Newton polynomials in Eq. (4.40) that they are ideal for iterative methods.

The Newton interpolation of a scalar function is very robust numerically, provided that two stabilizing procedures are followed. These pertain to the particular way in which the sampling points $\left\{z_{k}\right\}$ are chos, n. First, the points should be chosen uniformly from the unit circle, or some mapping of the unit circle. From complex analysis, it can be shown [42] that such uniform sampling gives uniform convergence of the interpolation on $D$. As such, this expansion is sometimes called the uniform polynomial approximation. Second, the sampling points must be staggered with respect to the order in which they are taken. This is to minimize numerical linear dependence among the functions $\left\{R_{k}(z)\right\}$. The theory behind these procedures is discussed quite thoroughly by Berman et al. in Ref. 40 .

If the sampling points are chosen uniformly on the upper half circle and projected down onto the real axis, they are Gauss-Chebyshev quadrature points. To 
see this, we recall that the Chebyshev polynomials can be written as $T_{n}(\cos \theta)=$ $\cos (n \theta)$, where $\theta \in[0, \pi]$ is the phase which sweeps out the upper half circle. The set of $n$ Gauss-Chebyshev quadrature points is obtained by setting $T_{n}\left(x_{k}\right)=0$, and solving for $x_{k}=\cos \theta_{k}$. From the formula above, it is clear that $\left\{\theta_{k}\right\}$ satisfy $\theta_{k}=$ $\pi(2 k+1) / 2 n$ for $k=0, \ldots, n-1$. Since the phases depend linearly with $k$, they are uniformly distributed. It can be shown [43] that a Newton expansion with GaussChebyshev sampling points is identical to the Chebyshev polynomial expansion. The Chebyshev polynomials are, in fact, the most uniform polynomials. That is, they are the only classical orthogonal polynomials on $D=[-1,+1]$ with the property that the maximum absolute value they take is uniform throughout $D$. This fact is the origin of their great utility. (E.g. Legendre polynomials peak near the endpoints of $D$, and thus would give relatively poor interpolation accuracy near the center of $D$.) Thus, the Chebyshev polynomial expansion is a special case of the uniform Newton interpolation scheme when $D$ is a line segment.

To use the Newton interpolation polynomials for a matrix expansion, one would shift and scale the matrix so that its eigenvalues fall within the unit circle (or some mapping of the unit circle), i.e. the domain $D$. This procedure enhances numerical stability and efficiency. If the unscaled eigenvalues fall outside $D$, the expansion would rapidly diverge. The scaling makes the Newton expansion an interpolation scheme (rather than an extrapolation). If the unscaled eigenvalues fall within $D$, but in a very small portion of $D$, the scaling expands the spectrum to fall just inside $D$. This makes the already stable calculation more efficient by interpolating only the relevant spectral region.

The matrix expansion can be viewed as several simultaneous scalar expansions where the scalars correspond to eigenvalues. The only information required is the action of the matrix on a vector, and the spectral range of the matrix. The uniform polynomial approximation gives uniform accuracy throughout the entire spectral range. This is in contrast to the KSM, which use not only the matrix but also the right hand side vector. Indeed, the accuracy of the KSM is not uniform throughout the spectrum, but rather is greater in the more dynamically relevant region. Dynamical relevance is measured in terms of the overlap of the right hand side vector and 
the Hamiltonian eigenvectors. This means that the Newton expansion is not strictly the most efficient method, since it does not use the spectral decomposition of the right hand side vector for importance sampling. However, the Newton expansion can be more automated. That is, given the spectral range of the system matrix and the convergence properties of the relevant scalar interpolations, the convergence properties of the matrix expansion are known. This is not so for the KSM. Thus, although the KSM may be more efficient in terms of computer time, the Newton expansion is more automatic and may be more efficient in terms of human time.

We note that there is a version of the Newton expansion which uses nonuniform sampling, analogous to preconditioning the Newton expansion. It can be shown [44] that a Newton expansion using sampling points obtained as eigenvalues from a KSM calculation is mathematically equivalent to using the original KSM to obtain the solution. If relatively low accuracy is desired, this non-uniform polynomial approximation, called the residuum method, may be preferable. However, for higher accuracy, the uniform and non-uniform sampling usually have comparable efficiency [45], with the uniform sampling being more automated. For this reason, we use the uniform polynomial approximation exclusively in this dissertati $n$.

We now discuss the sampling region relevant for solving the ABC system. The ABC Green's function is analytic, as a function of the total energy $E$, in the upper half plane - as is the formal Green's function. If the Hamiltonian supports no bound states, the $A B C$ Gretn's function is also analytic on the real energy axis. Thus, it formally has a polynomial expansion for real total energies. Since the poles of $\mathbf{G}(E)$ are in the lower half plaue, as are the eigenvalues of $\mathbf{Z}$, the sampling points must enclose a region in the lower half plane. Were we to use a finite, constant absorbing potential, i.e. $\epsilon(\mathbf{q})=\epsilon$, the eigenvalues of $\mathbf{Z}$ would lie on a line segment in the lower half plane. With the proper shift and scale of $\mathbf{Z}$, the Chebyshev expansion could be used to represent the ABC Green's function. However, once $\epsilon(\mathbf{q})$ is coordinate dependent, the eigenvalues of $\mathbf{Z}$ have varying imaginary parts. Even if a line segment $D$ were placed in the densest region of the spectrum, most of the eigenvalues of $\mathbf{Z}$ would lie above or below $D$. In effect, we would be using an extrapolation scheme, which is known to perform poorly at best, and to diverge in most cases. The Chebyshev 
expansion is, thus, strictly inappropriate for the present problem. We must utilize, instead, the more general prescription provided by the Newton interpolation scheme.

Based on the analyticity of the ABC Green's function, the sampling points $\left\{z_{k}\right\}$ are chosen from the lower half circle pushed slightly below the real axis, i.e.

$$
z_{k}=\left\{\begin{array}{ll}
\cos \theta_{k}-i \delta, & \theta_{k} \in[0, \pi] \\
e^{i \theta_{k}}-i \delta, & \theta_{k} \in[\pi, 2 \pi]
\end{array},\right.
$$

where $\delta$ must be small enough to include in $D$ the eigenvalues closest to the real axis. In Eq. (4.44), the staggered angles are chosen by

$$
\theta_{k}=\bmod \left\{\frac{2 \pi}{K}\left[k+\frac{K}{n_{\text {stag }}} \bmod \left(k, n_{\text {stag }}\right)\right], 2 \pi\right\}
$$

where $k=0,1, \ldots, K-1$. In Eq. (4.45), the parameter $n_{\text {stag }}$ determines the amount of staggering. It is the number of sampling points chosen during each "pass" around the circle. Setting $n_{\text {stag }}=1$ or $K$ produces no staggering. We have found $n_{\text {stag }}=4$ or 8 to give the best results in the present calculations. Figure 4.4 demonstrates the sampling points using $K=64, n_{\text {stag }}=4$, and $\delta=0$. The lines in the interior of $D$ manifest the staggering, in which four points per pass are taken. The points on the real axis are Gauss-Chebyshev quadrature points.

We now examine the convergence properties of the Newton polynomial expansion of $g(z)=(E-z)^{-1}$. We need to choose a typical total energy $E$, and a typical eigenvalue $z$. In this choice there are two distinct limits, namely that $|E-z|$ is relatively small or large. Clearly in the first case, the polynomial expansion behaves poorly, and in the second it may behave well. To guide us in making relevant choices, we recall that the matrix $\mathbf{Z}$ is shifted and scaled so that the lower half unit circle just encloses the spectrum of $\mathbf{Z}$. Thus, all values of $E$ and $z$ are unitless for the present purposes. Since we plan to use a grid representation which usually contains large kinetic energy components, $E$ is typically just above -1 . We use $E=-0.8$.

All tests reported in this paragraph use 64 support points, staggered by $n_{\text {stag }}=4$. The abscissa is the number of Newton polynomial terms taken, and the ordinate is the common logarithm of the relative error from the exact result. For the first test, we assume that we can use a very strong absorbing potential. In that 


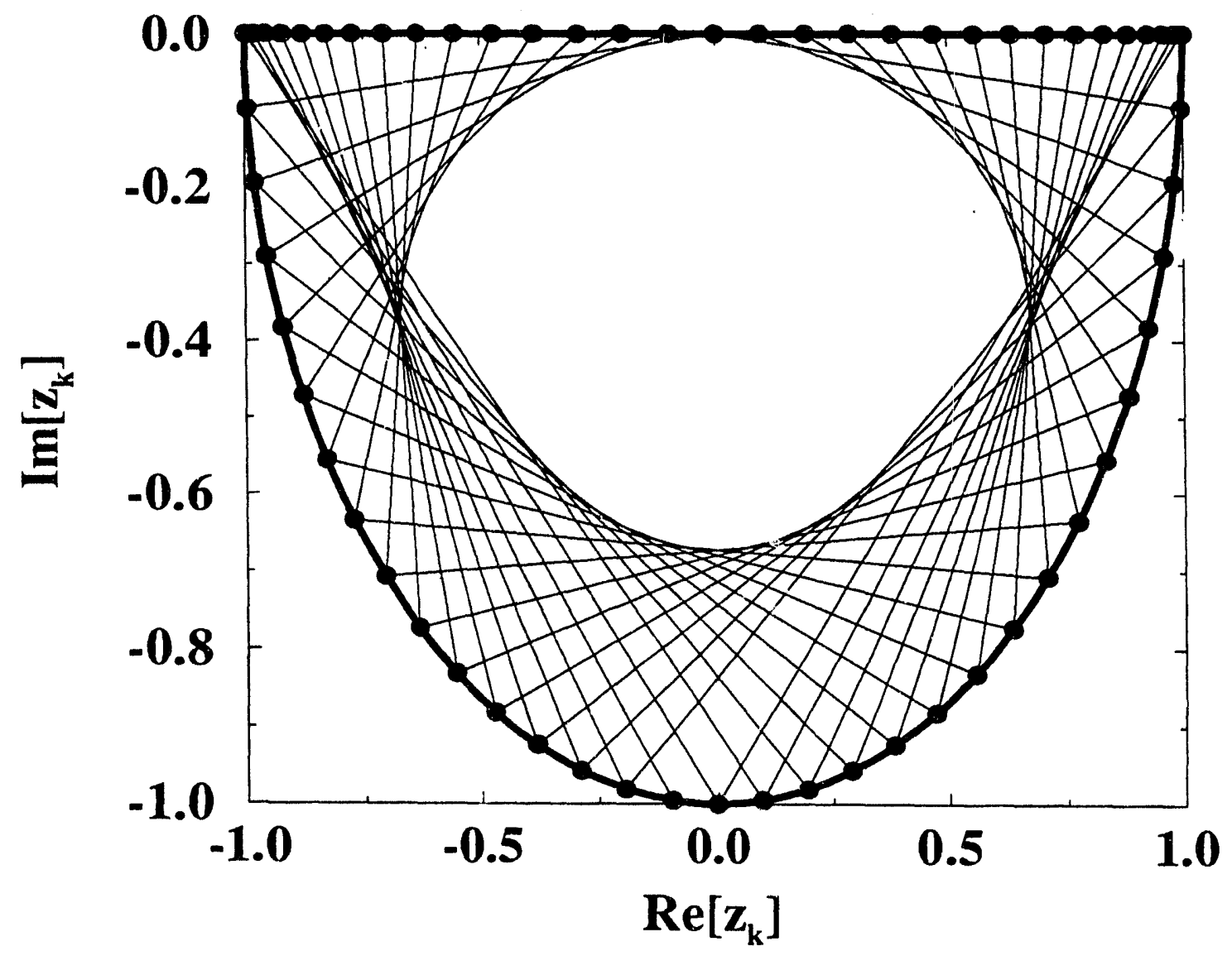

Figure 4.4: The Newton interpolation sampling points used to represent the eigenvalue spectrum of the ABC linear system. This sampling uses $K=64$ points shifted down from the real axis by $\delta=0$. The lines in the interior manifest the staggering. At each pass around the circle, $n_{\text {stag }}=4$ points are taken. The points on the real axis, which are projected down from the upper half circle, are Gauss-Chebyshev quadrature points. 
case, the eigenvalues of $\mathbf{Z}$ are pushed far below the real axis, and $\delta$ can be quite large. Using $\delta=5$ and $z=-5.5 i$, the convergence of the Newton expansion is shown as the dashed line in Fig. 4.5. We see that machine accuracy is obtained using 18 Newton terms. The subsequent oscillations are the result of numerical round off error. Next we consider the effect of a more moderate absorbing potential, in which $\delta=0.4$ and $z=-0.9 i$. In this case, the domain $D$ is closer to the pole at $z=E$. Although formally $g$ is still analytic on $D$, the convergence behavior may be sensitive to the presence of the pole. The convergence for this case is shown as the solid line in Fig. 4.5. We see that although the expansion is clearly converging, it does so less rapidly than in the first case. Also, it shows systematic oscillations, resulting from the effect that the pole has on every fourth sampling point and Newton term. Thus, we conclude that the Newton expansion converges more rapidly with a stronger absorbing potential. This is qualitatively similar to the time domain, in which a stronger absorbing potential absorbs a wavepacket more rapidly in time (also see Chapter 3 ). In additon, we conclude that the pole can perturb the convergence properties of the Newton expansion of $g(z)=(E-z)^{-1}$.

To determine whether the Newton expansion of $\mathbf{g}(\mathbf{Z})=(E-\mathbf{Z})^{-1}$ is a feasible solution to the $A B C$ system, we examine the eigenvalue spectrum of $Z$. If eigenvalues of $\mathrm{Z}$ are not too close to $E$, a direct expansion is feasible. Otherwise, an alternative method may be preferable. Figure 4.6 shows the shifted and scaled eigenvalue spectrum of $\mathrm{Z}$ for collinear $\mathrm{H}+\mathrm{H}_{2}$ at $E=0.99 \mathrm{eV}$ (cf. Figs. 4.1 and 4.2). The scaled and shifted total energy, $E=-0.789$, is marked by the " $\times$ " on the real axis. In this case, the eigenvalue closest to $E$ is 0.0238 scaled energy units from the pole. Thus, the $A B C$ eigenvalues can be quite close to both the real axis in general and the pole at $z=E$.

We note that this eigenvalue spectrum is remarkably similar to those found in complex coordinate scaling theory [46]. We also see that assignments can be made at low enough (real and imaginary) energy, but at higher energies meaningful assignments are difficult to make. The various progressions become mixed by the anharmonicity and coupling between modes. It is also interesting that, although the scaling procedure expands the spectrum to approximately fill the lower half circle, 


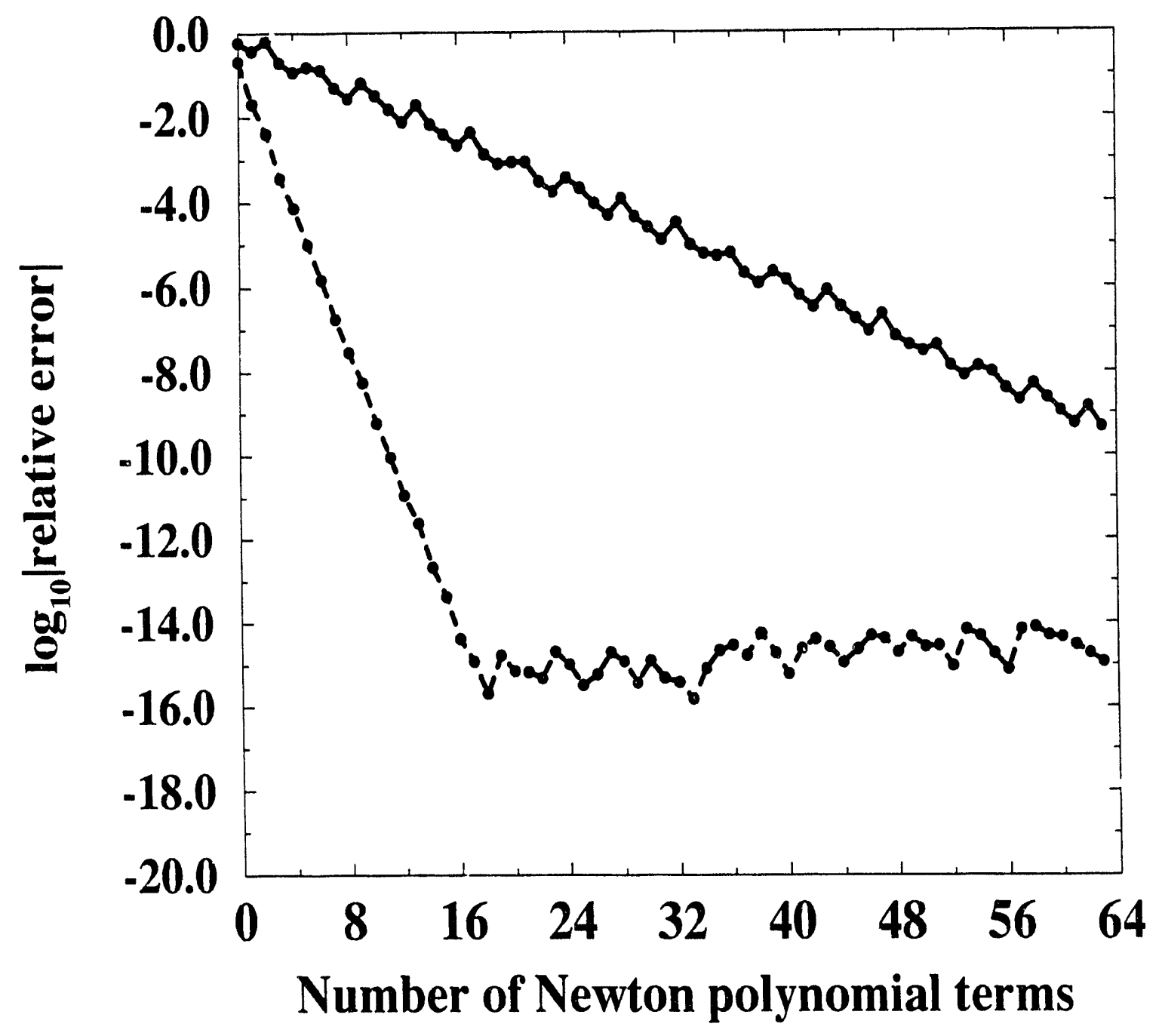

Figure 4.5: Newton expansion of the function $g(z)=(E-z)^{-1}$ for $E=-0.8$. In all calculations, 64 support points are used, staggered by $n_{\text {stag }}=4$. The dashed plot corresponds to a strong absorbing potential, using $\delta=5$ and $z=-5.5 i$. Here we see rapid convergence, obtaining machine accuracy with 18 Newton terms. The solid plot corresponds to a moderate absorbing potential, using $\delta=0.4$ and $z=-0.9 i$. In this case, convergence is less rapid, presumbaly because the pole at $z=E$ is closer to $D$. In addition, the convergence in the second case shows oscillations whenever a sampling point comes close to the pole. 


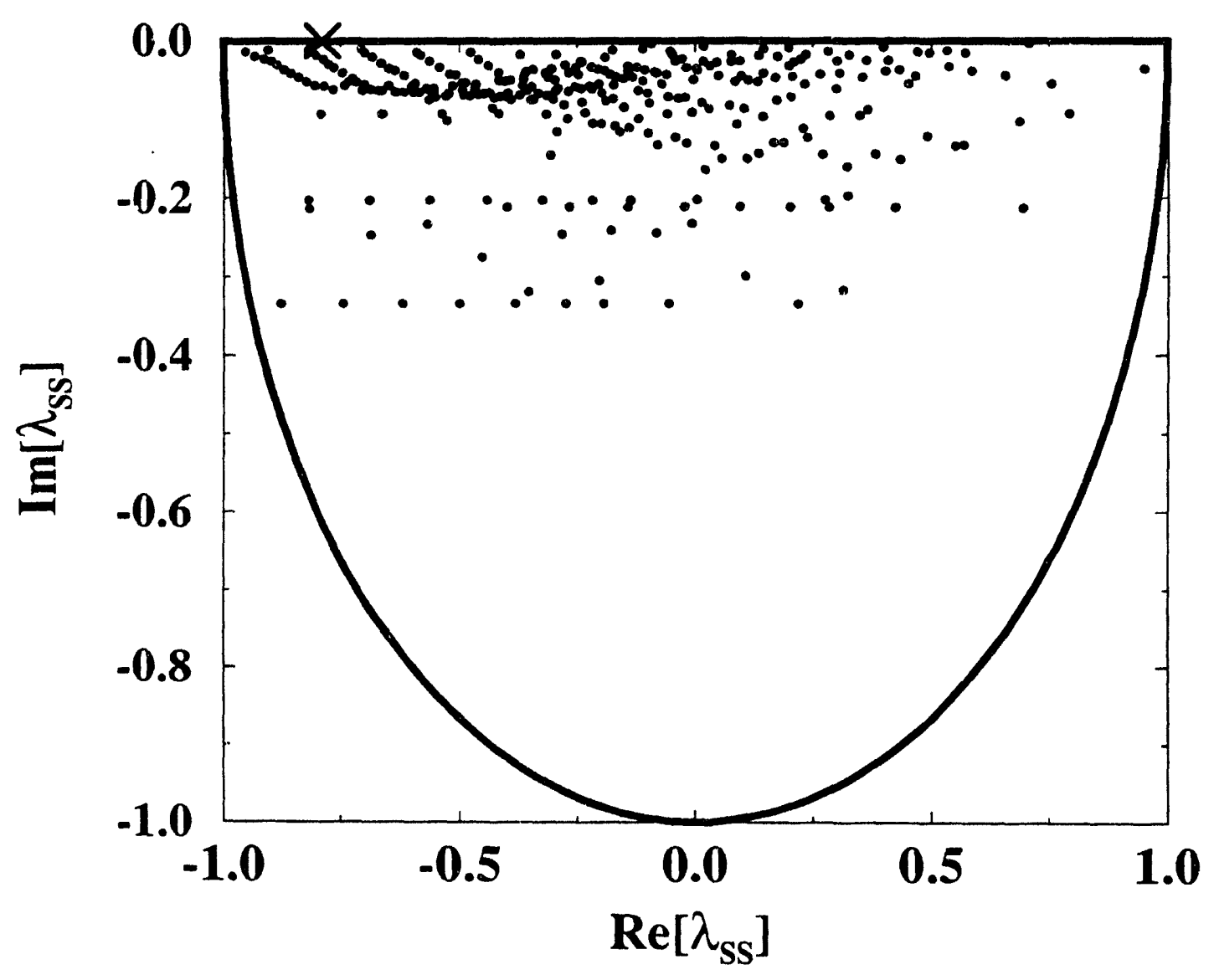

Figure 4.6: The scaled and shifted $\mathrm{ABC}$ spectrum for collinear $\mathrm{H}+\mathrm{H}_{2}$ at $E=0.99 \mathrm{eV}$. $\left\{\lambda_{S S}\right\}$ are the scaled and shifted eigenvalues of $\mathbf{Z} \equiv \mathbf{H}-i \epsilon$. The scaled and shifted total energy, $E=-0.789$, is marked by the " $x$ " on the real axis. The eigenvalue spectrum shows structure reminiscent of that found in complex coordinate scaling theory. Assignments can be made at low enough (real and imaginary) energy, but at higher energies meaningful assignments are difficult. The eigenvalue closest to $E$ is 0.0238 scaled energy units from the pole. 
there is still much empty space. That is, a more efficient representation of this reaction would be provided by a sampling domain which is more rectangular [40]. Such optimization may prove beneficial for large scale calculations.

The results in Fig. 4.6 present a potential difficulty in applying the Newton expansion to solve the $\mathrm{ABC}$ system. To solve the $\mathrm{ABC}$ system portrayed in Fig. 4.6, the sampling domain must both exclude the pole at $z=-0.789$ and include the eigenvalue at $z \cong(-0.789)+i(-0.0238)$. The difficulty pertains to the choice of $\delta$, the parameter which controls the downward shift of the sampling domain $D$ from the real axis. In general, if $\delta$ is too small (a cautious choice), the expansion may converge too slowly because of the strong influence from the pole. Alternatively, if $\delta$ is too large (an adventurous choice), the expansion diverges rapidly because $D$ does not include the entire spectrum of $\mathbf{Z}$. The proper choice of $\delta$ is thus absolutely crucial. The optimal choice would be $\delta=-\operatorname{Im}\left[\lambda_{A B C}\right]$, where $\lambda_{A B C}$ is the complex eigenvaluc closest to the real axis.

To examine the sensitivity of the convergence behavior to the precise placement of the sampling domain, convergence tests are performed with various values of $\delta$. In what follows, 1024 support points staggered by $n_{\text {stag }}=8$ are used to expand $g(z)=(E-z)^{-1}$ for $E=-0.8$ and $z=(-0.8)+i(-0.024)$. Figure 4.7 shows the convergence behavior for three values of $\delta$. The dotted line is the most cautious choice, $\delta=0.012$. Accordingly, the expansion is stable with slow convergence. The dashed line is a more optimal choice, $\delta=0.02$. The corresponding convergence is clearly much more rapid. The solid line is the most adventurous choice, $\delta=0.07$. The sampling domain excludes $z$ with this choice, and the expansion diverges. Previous studies have shown that it may be very difficult to determine accurately complex eigenvalues with small imaginary parts for large systems $[29,30]$. Thus, this fact coupled with the extreme sensitivity to the choice of $\delta$ makes solving the ABC system with the Newtor expansion seem unfeasible.

We have shown that the convergence behavior of the Newton expansion for $\mathbf{g}(\mathbf{Z})=(E-\mathbf{Z})^{-1}$ is extremely sensitive to the eigenvalue of $\mathbf{Z}$ closest to the real axis, denoted by $\lambda_{A B C}$. This has made it unfeasible to solve the $\mathrm{ABC}$ system with the Newton expansion. We can make great progress, however, by physically analyzing 


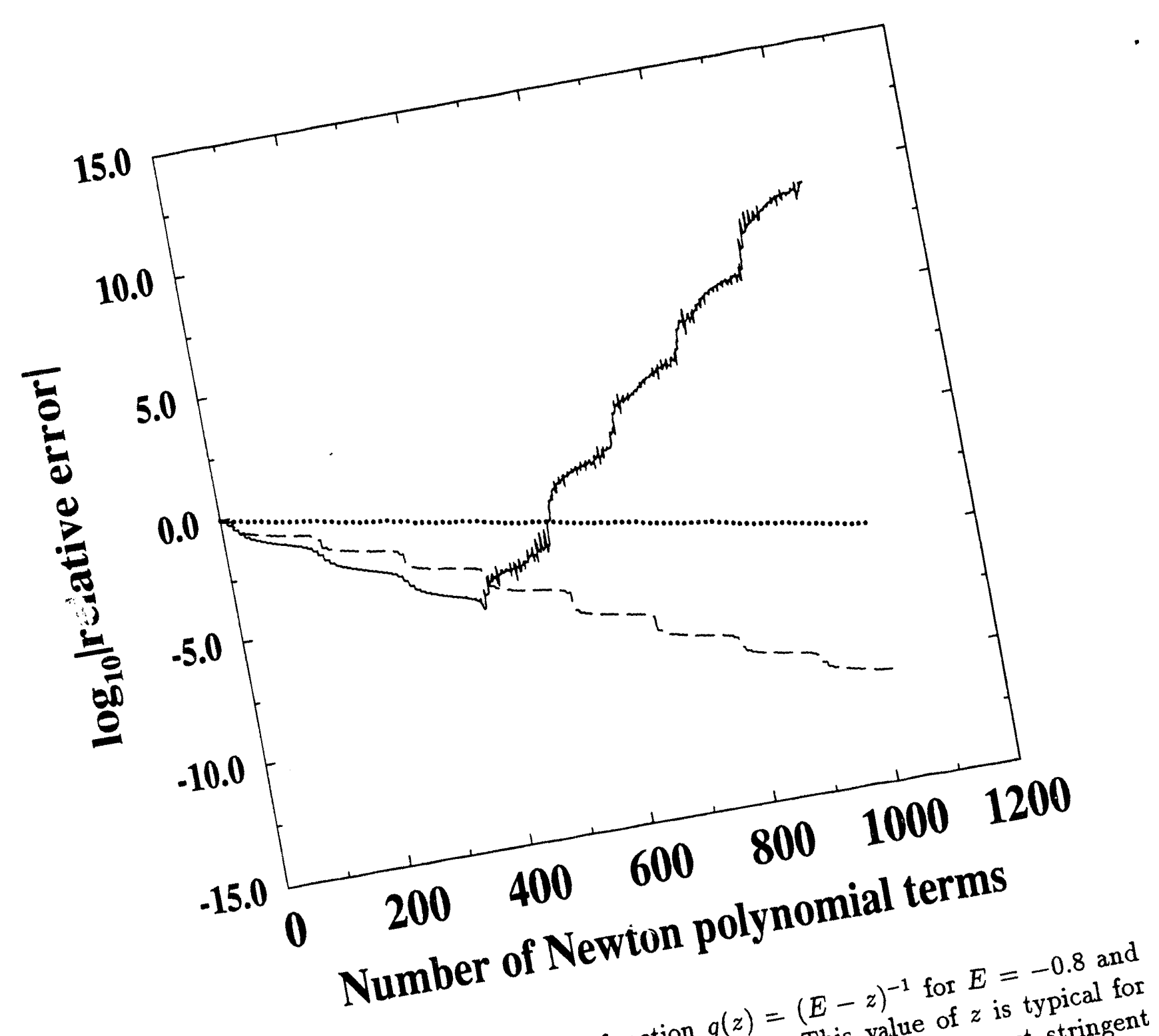

Figure 4.7: Newton expansion of the function $z=(-0.8)+i(-0.024)$, and close to the reamion. In all calculatich $\delta=0.012$, shows eigenvalues of $Z$ wergence of the Newton. The dotted plot, $\delta=0.02$, shows more rapiain test for the converged stagered by $n_{\text {stag }}$ dashed plot, for excludes $z$ from the sampling choice of $\delta$. points are used convergence. Ih which $\delta=0.07$, excludes $z$ fitive to the choice of $\delta$. stable but slowe The solid plot, for wehavior is thus very senstive convergence. The convergence behavior is thes. 
the meaning of this numerical result.

In formal scattering theory, the complex energies $E_{\text {res }}$ for which $\hat{G}^{+}\left(E_{\text {res }}\right)$ diverges correspond to resonances, i.e. collision complexes. The real part of $E_{\text {res }}$ locates the position of the resonance in energy space, and the imaginary part of $E_{\text {res }}$ determines the lifetime of the complex. Defining $\Gamma_{\text {res }}=-2 \operatorname{Im}\left[E_{\text {res }}\right]$, the lifetime is given by $\tau_{\text {res }}=\hbar / \Gamma_{\text {res }}$. Thus, a resonance energy close to the real axis indicates a long lived collision complex. In the ABC formulation, all the eigenvalues of $\mathbf{Z}$ are complex energies for which $\mathrm{G}(E)$ diverges. However, most (or all) of these do not correspond to physical resonances, but rather control how much time is required for reaction and absorption. The total time for the reaction in the ABC formulation is determined by the eigenvalue of $\mathbf{Z}$ closest to the real axis, i.e. $\lambda_{A B C}$. Thus, $T_{A B C}=\hbar / \Gamma_{A B C}$ is the total time for reaction and absorption, where $\Gamma_{A B C}=-2 \operatorname{Im}\left[\lambda_{A B C}\right]$.

We use this time dependent picture to construct a useful computational framework for solving the $A B C$ system. First, we recall the Fourier integral representation of the ABC Green's function (see also Chapter 3):

$$
\mathbf{G}(E)=(i \hbar)^{-1} \int_{0}^{\infty} d t e^{i(E-\mathbf{Z}) t / \hbar} .
$$

Because the magnitude of $\epsilon$ is finite in the $\mathrm{ABC}$ formulation, this integral converges in finite time (as opposed to the infinite time required by formal scattering theory). Second, we use the Newton expansion to represent the exponential in Eq. (4.46). Since $\mathbf{u}(\mathbf{Z})=e^{-i \mathbf{Z}_{t} / \hbar}$ is an entire analytic function [i.e. $\mathbf{u}(\mathbf{Z})$ has no poles], we can choose $\delta=0$ to define the sampling region.

We still need to determine $T_{A B C}$, which by the above analysis is tantamount to determining $\lambda_{A B C}$. This was the stumbling block discovered above in the time independent expansion. However, determining $T_{A B C}$ is trivial in the time domain. It is simply the finite time required to evaluate the ABC Green's function in Eq. (4.46), determined by monitoring the exponential decay of the integrand and stopping the calculation when the integrand becomes negligible. In this way, we can solve the $\mathrm{ABC}$ system with an algorithm which is accurate, has a fixed and small memory requirement, and is guaranteed to converge. We pursue this "indirect" polynomial expansion for the ABC Green's function in the next Section. 
Before proceeding with the description of the "indirect" polynomial expansion, we pause to ask: Why is there no computationally tractable direct polynomial expansion for the ABC Green's function? To answer this, we note that the ABC formulation was originally derived [13] to exploit the fact that a reactive collision results from forces which are highly localized in space. However, none of the approaches discussed above for solving the $\mathrm{ABC}$ system exploit the fact that the collision event is also local in time. Indeed, the inverse operator implicitly accounts for infinite time dynamics [cf. Eq. (4.46)], which physically speaking, is the source of the pole. By passing to the time domain, we can fully exploit the finite time nature of the collision event. And by construcing only finite time dynamics, we effectively remove the pole, yielding a stable and accurate computational framework.

\subsubsection{Indirect Expansion}

We solve the $A B C$ system by numerical half Fourier transformation of the Newton expansion for the ABC propagator. This approach introduces certain convergence parameters which we describe below.

\section{Time Decomposition}

Beginning with Eq. (4.46), we decompose the time integral into a sum of terms involving relatively short time propagators, giving:

$$
\mathbf{G}(E)=(i \hbar)^{-1} \sum_{n=0}^{\infty} \int_{n \Delta t}^{(n+1) \Delta t} d t e^{i(E-\mathbf{Z}) t / \hbar}
$$

Changing integration variables $t \rightarrow t+n \Delta t$ gives

$$
\mathbf{G}(E)=(i \hbar)^{-1} \sum_{n=0}^{\infty}\left[e^{i E \Delta t / \hbar} e^{-i \mathbf{Z} \Delta t / \hbar}\right]^{n} \int_{0}^{\Delta t} d t e^{i E t / \hbar} e^{-i \mathbf{Z} t / \hbar} .
$$

With this decomposition, the infinite time integral in Eq. (4.46) is evaluated exactly by only explicitly integrating up to $\Delta t$. Two criteria determine the optimal choice for $\Delta t$. First, if $\Delta t$ is too large, numerical roundoff error precludes accurate interpolation of $\left\{\left(z_{k}, u_{k}\right)\right\}$ for $u(z)=e^{-i z \Delta t}$. That is because for long time, the $\left\{u_{k}\right\}$ for $\left\{z_{k}\right\}$ below the real axis are comparable to machine precision. Roundoff error dominates when 
a small $u_{k}$ is added to a $u_{k}$ of order unity. Second, if $\Delta t$ is too small the Newton expansion is not optimally efficient. Thus, the competition between stability and efficiency determines an optimal range for $\Delta t$.

\section{Scaling and Shifting}

We describe the details of shifting and scaling the matrix $\mathbf{Z}$. In addition to enhancing the stability and efficiency of the expansion, as discussed above, the shift and scale also help to determine $\Delta t$. We begin by rewriting the $\mathrm{ABC}$ propagator as

$$
e^{-i \mathbf{Z} \Delta t / \hbar}=e^{-i(\mathbf{Z}) \Delta t / \hbar} e^{-i \mathbf{Z} \cdot \boldsymbol{\theta}}
$$

where

$$
\mathbf{Z}_{s}=\frac{\mathbf{Z}-\langle\mathbf{Z}\rangle}{\Delta \mathbf{Z} / 2}
$$

In Eqs. (4.49) and (4.50), the following quantities are:

$$
\begin{array}{ll}
t_{s} & =\Delta t \Delta \mathbf{Z} / 2 \hbar \\
\langle\mathbf{Z}\rangle & =\left[\operatorname{Re}\left(\lambda_{\max }\right)+\operatorname{Re}\left(\lambda_{\min }\right)\right] / 2 \\
\Delta \mathbf{Z} / 2 & =\max \left\{\left|\lambda_{\max }-\langle\mathbf{Z}\rangle\right|,\left|\lambda_{\min }-\langle\mathbf{Z}\rangle\right|\right\},
\end{array}
$$

where $\lambda_{\max }$ and $\lambda_{\min }$ are the eigenvalues of $\mathbf{Z}$ with largest and smallest real part, respectively. In the case of a positive definite Hamiltonian matrix $\mathbf{H}$ and a weak absorbing potential $\epsilon,\left|\lambda_{\min }\right| \cong 0$ and the above relations simplify to $\langle\mathbf{Z}\rangle=\Delta \mathbf{Z} / 2=$ $\operatorname{Re}\left(\lambda_{\max }\right) / 2$. In practice, $\lambda_{\min }$ and $\lambda_{\max }$ are determined with a low-order Lanczos calculation [26].

In actual calculations, $e^{-i \mathbf{Z}_{*} t_{0}}$ is the matrix which is expanded in Newton polynomials according to

$$
e^{-i \mathbf{Z}_{s} t_{\bullet}} \cong \sum_{k=0}^{K} a_{k}\left(t_{s}\right) R_{k}\left(\mathbf{Z}_{s}\right),
$$

and Eqs. (4.39-4.45). The number of terms required for convergence is determined directly by $t_{s}$, not by $\Delta t$. Given the spectral range $\Delta \mathbf{Z}$ and the optimal scaled time $t_{s}$, the optimal time step $\Delta t$ is obtained from Eq. (4.51). 
We obtain the relationship between $t_{s}$ and the number of Newton terms required to interpolate to a certain relative error, e.g. $10^{-3}$. Denoting that funciion by $K_{\text {opt }}\left(t_{s}\right)$, we seek the $t_{s}$ dependence of $f_{\text {newt }} \equiv K_{\text {opt }}\left(t_{s}\right) / t_{s}$. The optimal scaled time is given by the shortest time for which $f_{\text {newt }}$ reaches a minimum value. To obtain this, we examine the convergence properties of the Newton expansion for the scalar function $u(z)=e^{-i z t_{\text {}}}$ for several values of $t_{\mathbf{s}}$. Figure 4.6 shows that a value of $z$ which is characteristic of the $\mathrm{ABC}$ spectrum is $z=(-0.5)+i(-0.1)$. All the calculations reported in this paragraph use 256 support points staggered by $n_{\text {stag }}=8$. Figure 4.8 shows the convergence of interpolations using $t_{s}=10,20,30, \ldots, 100$. We see that in order to attain a certain accuracy, more Newton terms are required for longer times. That is because more sampling points and polynomial terms are required to represent the more rapid oscillations in the longer time exponentials. We also see the effect of roundoff error for longer times, causing the expansion to converge to progressively less accurate values. The dashed horizontal line marks the $10^{-3}$ accuracy level. Using this accuracy to obtain $f_{\text {newt }}$ as a function of $t_{s}, f_{\text {newt }}$ reaches the minimum value of 1.6 at $t_{s}=50$. Thus, $t_{s}=50$ is optimal, requiring $K_{\text {opt }}\left(t_{s}=50\right)=80$ terms. Based on these results, we use $t_{s}=50$ as the optimal scaled time for all scattering calculations reported in this dissertation. It is significant that this scaled convergence result is not dependent on the physical system under study, but rather is a fact of scalar interpolation. As such, no re-calibration is necessary when applying the method to different physical systems.

\section{Time Integration}

The time dependent picture requires evaluating the time integral which Fourier transforms the time Green's function to the energy Green's function. This is a very small price to pay. This integration can be performed with essentially no approximation or additional effort. Indeed, after representing the ABC propagator with the Newton expansion, the integral factor in Eq. (4.48) becomes

$$
\int_{0}^{\Delta t} d t e^{i E t / \hbar} e^{-i \mathbf{Z} t / \hbar} \cong \sum_{k=0}^{K} b_{k}(E, \Delta t) R_{k}\left(\mathbf{Z}_{s}\right)
$$




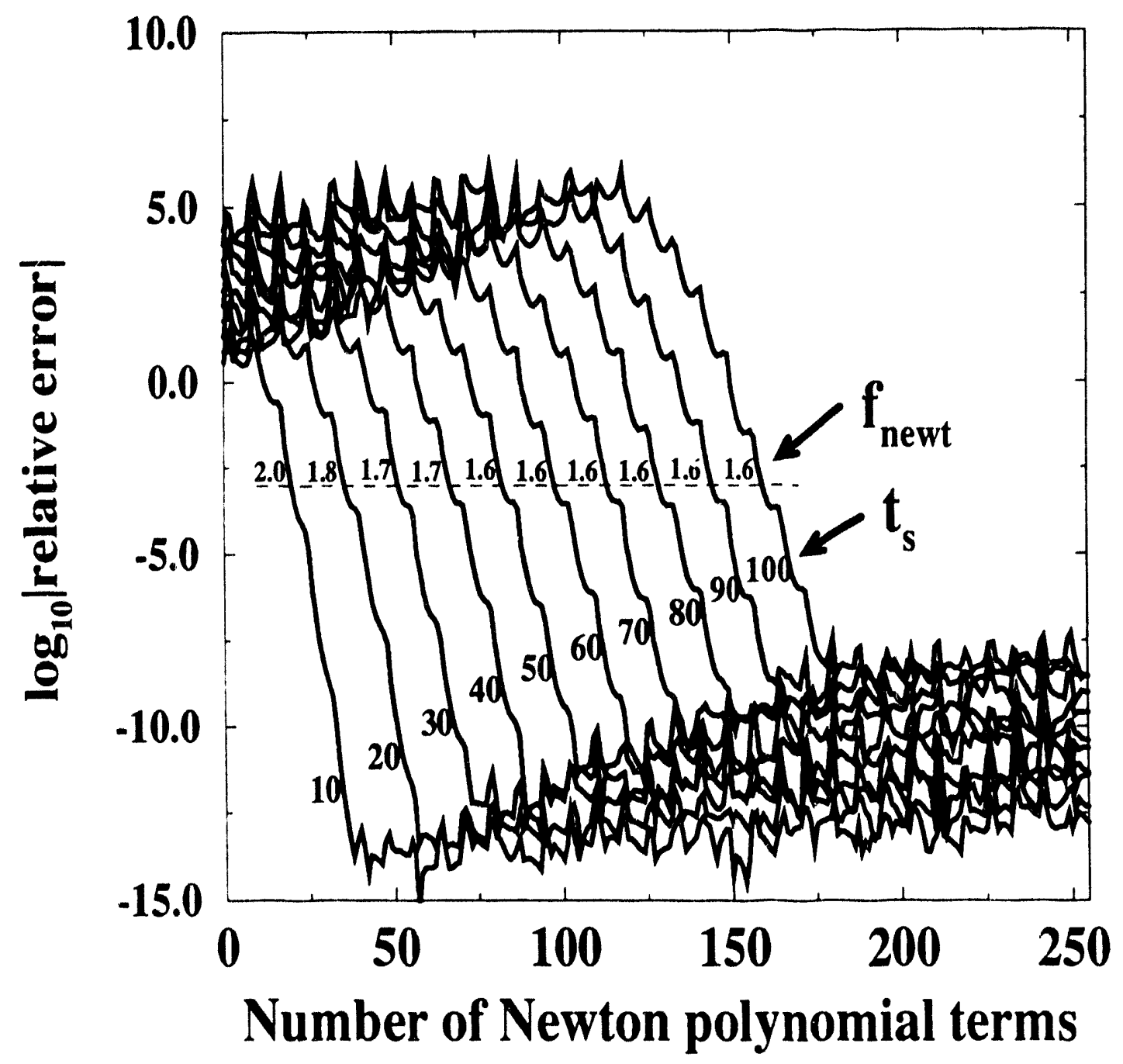

Figure 4.8: Convergence of the Newton expansion for $u(z)=e^{-i z t}$ using $z=(-0.5)+$ $i(-0.1)$, and for several values of $t_{s}$. More terms are required for longer times to represent the more rapid oscillations in the exponential function. Roundoff error is more prevalent for longer times, giving progressively less accurate results. The optimal time is $t_{s}=50$, the shortest time for which $f_{\text {new }}$ reaches the minimum value of 1.6 . 
where

$$
b_{k}(E, t) \equiv \int_{0}^{t} d \tau e^{i(E-\langle\mathbf{Z}\rangle) \tau / \hbar} a_{k}(\tau \Delta \mathbf{Z} / 2 \hbar) .
$$

The integral in Eq. (4.54) must be evaluated numerically. Once the expansion coefficients $\left\{a_{k}\left(t_{s}\right)\right\}$ are known, Gaussian quadrature with $N_{\text {quad }}$ points can be used to obtain the energy dependent coefficients exactly.

\section{Summary and the Residual}

Using the same polynomial expansion for the other exponentials in Eq. (4.48), the ABC Green's function becomes

$$
\mathrm{G}(E) \cong(i \hbar)^{-1} \sum_{n=0}^{\infty}\left[\sum_{k=0}^{K} c_{k}(E, \Delta t) R_{k}\left(\mathbf{Z}_{s}\right)\right]^{n} \times \sum_{k^{\prime}=0}^{K} b_{k^{\prime}}(E, \Delta t) R_{k^{\prime}}\left(\mathbf{Z}_{s}\right)
$$

where

$$
c_{k}(E, t) \equiv e^{i(E-\langle\mathbf{Z}\rangle) t / \hbar} a_{k}(t \Delta \mathbf{Z} / 2 \hbar) .
$$

Eqs. (4.55) and (4.56) give the working expressions in the time dependent solution of the $\mathrm{ABC}$ system.

To summarize, we construct the RHS vector of the linear system in Eq. (4.22). Next, we choose sampling points $\left\{z_{k}\right\}$ in the region where the eigenvalues of $\mathrm{Z}_{s}$ are likely to be [cf. Eqs. (4.44) and (4.45)]. A set of scalar interpolations is then performed using $u(z)=e^{-i z t}$, each for a different time as required to converge the integral in Eq. (4.54). With the energy dependent coefficients $\left\{b_{k}(E, \Delta t)\right\}$ computed, we apply the matrix in Eq. (4.53) (i.e. the Fourier transform up to $\Delta t$ ) to the starting vector. This is done in the standard fashion, in which the successively higher powers of $\mathbf{Z}_{s}$ are accumulated recursively, thereby requiring a minimal amount of core memory. This yields a new vector, to which the remaining exponential matrices are applied.

Convergence of the first sum in Eq. (4.55) is monitored by examining the modulus of the vectors being summed. We define the partially summed $A B C$ wavefunction as

$$
\Psi_{\mathbf{n}_{r}}^{N}(E)=(i \hbar)^{-1} \sum_{n=0}^{N} \mathbf{v}_{n}
$$


where the summand vector is given by

$$
\mathbf{v}_{n}=\left[\sum_{k=0}^{K} c_{k}(E, \Delta t) R_{k}\left(\mathbf{Z}_{s}\right)\right]^{n} \times \sum_{k^{\prime}=0}^{K} b_{k^{\prime}}(E, \Delta t) R_{k^{\prime}}\left(\mathbf{Z}_{s}\right) i \boldsymbol{\epsilon} \boldsymbol{\Phi}_{\mathbf{n}_{r}}(E) .
$$

The residual is defined as

$$
r_{N} \equiv \frac{\left\|\mathbf{v}_{N}\right\|}{\left\|\Psi_{\mathbf{n}_{r}}^{N}(E)\right\|}
$$

When $r_{N}$ falls below the input accuracy tolerance denoted by $\delta$ (not to be confused with the downward shift of the sampling domain, which is now set to zero), the calculation is stopped and the reaction probability computed using Eq. (4.20) in the finite basis. The resulting total propagation time gives an operational definition for $T_{A B C}$, as discussed above. This procedure is not only straightforward, but also gives the desirable feature that the input accuracy tolerance $\delta$ is almost identical to the resulting error in the reaction probability.

\subsection{Quantum Reactive Scattering Calculations}

We illustrate the accuracy and convergence properties of the time dependent solution to the $\mathrm{ABC}$ system by performing IRP calculations for three dimensional $\mathrm{D}+\mathrm{H}_{2}(v, j) \rightarrow \mathrm{DH}+\mathrm{H}$ with zero total angular momentum. We defer all discussion of the basis set, asymptotic state, and absorbing potential used for the present calculations. Thorough descriptions of these are given in the following Chapter. Instead, we wish to focus on the convergence properties of the time dependent solution with respect to the three convergence parameters: $f_{\text {newt }}, N_{\text {quad }}$, and $\delta$. As such, when reporting error in scattering calculations, the error is measured from an ABC calculation which is well converged with respect to the parameter in question. By measuring error in this way, we subtract out the error from the $A B C$ approximation and focus only on the error incurred by using the time dependent solution.

\subsubsection{Reaction Probabilites}

To measure error in this way, we must first be sure that the $A B C$ reaction probabilities are converging to the correct reaction probabilities. Figure 4.9 shows 
the IRP for the $\mathrm{D}+\mathrm{H}_{2}(v, j)$ at $E=0.9 \mathrm{eV}$ for all the 14 energetically accessible initial states. The solid lines are obtained using the ABC Green's function method. The data in the dashed lines are from Groenenboom and Colbert [11], who used the $S$ matrix version of the Kohn variational principle [23] to obtain the entire $S$-matrix at this energy. The large probabilities are from the $v=0$ reactant vibrational state, and the smaller probabilities (multiplied by 20) are from $v=1$. The agreement is excellent for all initial states, even those for which the reaction probabilities are quite small, which are more difficult to converge with the ABC formulation.

The initial state $j=1$ reaction probabilities are the largest for both $v=0$ and 1. This phenomenon is also observed in studies of the $\mathrm{H}_{2}(v, j)+\mathrm{OH} \rightarrow \mathrm{H}+$ $\mathrm{H}_{2} \mathrm{O}$ reaction [47]. This can be explained by considering the role of orbital angular momentum and its classical counterpart, the impact parameter. For zero total angular momentum, the initial orbital angular momentum quantum number $l$ must equal the initial rotational quantum number $j$. The $j=0$ collision involves mostly low impact parameters, including the energetically unfavorable " $\mathrm{T}$ shaped" configuration of the three particles. The $j=1$ collision, on the other hand, couples most strongly to impact parameters greater than zero, avoiding the " $\mathrm{T}$ shaped" configuration and involving near collinear geometries more than $j=0$. Since these collinear geometries are more reactive, the $j=1$ state gives a larger reaction probability. Larger $j$ values couple to larger impact parameters, which give progressively smaller reaction probabilities as shown in Fig. 4.9.

These $\mathrm{ABC}$ calculations use $f_{\text {newt }}=1.6, N_{\text {quad }}=40$, and $\delta=4 \times 10^{-4}$. We study the convergence with respect to these parameters below.

\subsubsection{Convergence Tests}

\section{Degree of Expansion}

Using the optimal scaled time $t_{s}=50$ determined above, we demonstrate convergence of the reactive scattering calculations with respect to $f_{\text {newt }}$. Figure 4.10 shows the convergence of IRP calculations for the $\mathrm{D}+\mathrm{H}_{2}(v, j)$ system with the following initial states: $[E=0.9 \mathrm{eV}(v, j)=(0,0),(0,4)],[E=1.0 \mathrm{eV}(v, j)=(0,6),(1,4)]$ 


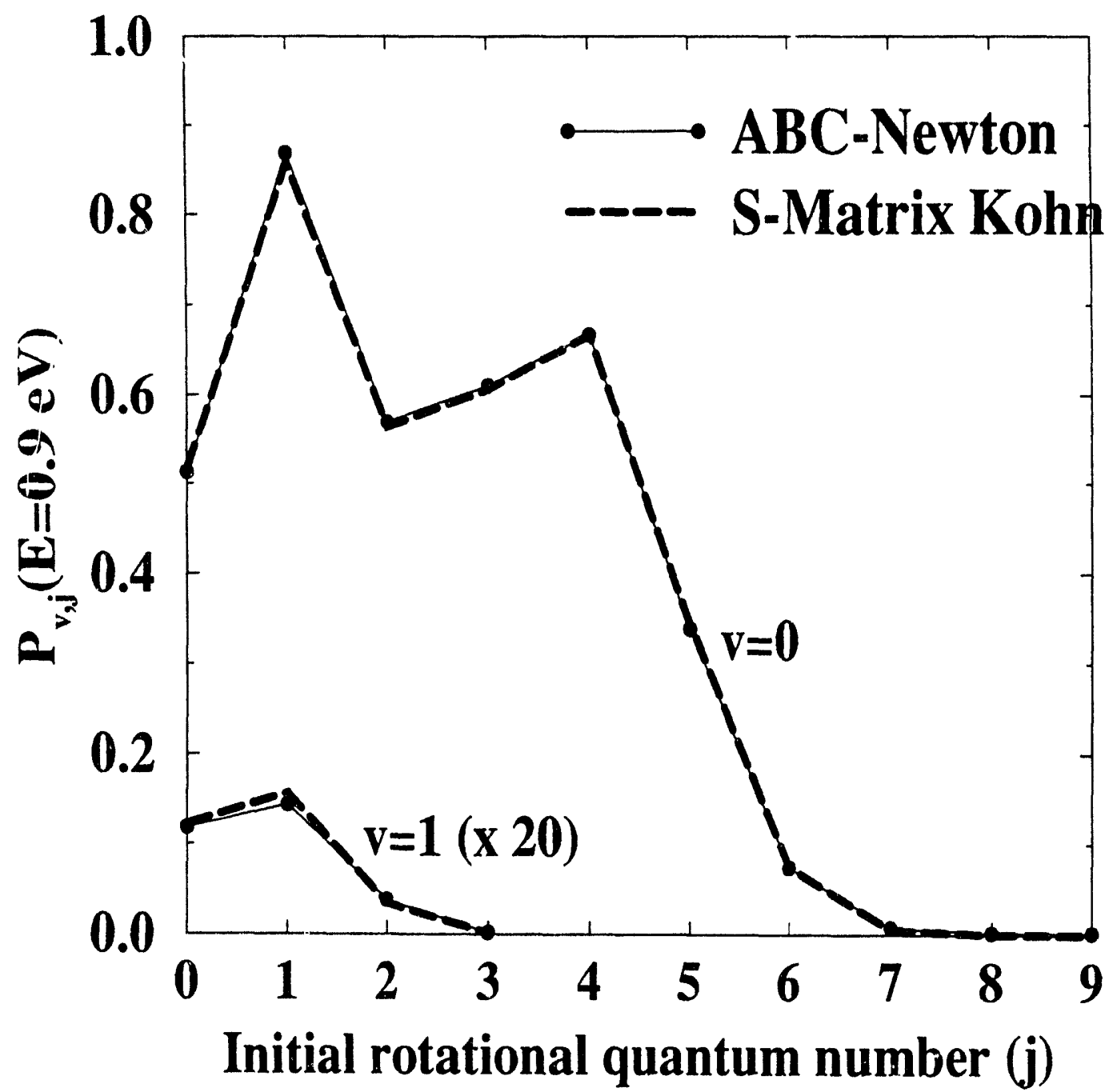

Figure 4.9: The initial state selected reaction probability for $\mathrm{D}+\mathrm{H}_{2}(v, j) \rightarrow \mathrm{DH}+\mathrm{H}$ at the total energy $E=0.9 \mathrm{eV}$, from all energetically accessible initial states. The solid lines are the present calculation, and the dashed lines are from the $S$-matrix Kohn variational principle calculations of Groenenboom and Colbert, in which the stateto-state reaction probabilities are summed for comparison. The larger probabilities are from the $v=0$ vibrational state, and the smaller (multiplied by 20) are from the $v=1$ vibrational state. Excellent agreement is obtained for all initial states, even those with small probabilities which are most challenging for the ABC method. 
and $[E=1.1 \mathrm{eV}(v, j)=(0,2),(1,2)]$. We note that for these calculations, $f_{\text {newt }}=1.5$ corresponds to 75 Newton terms and $f_{\text {newt }}=2.0$ to 100 terms. We see rapid convergence for all IRPs. In fact, the convergence is roughly independent of the system, i.e. energy and initial condition, being studied. In these examples, three digit accuracy can be obtained using $f_{\text {newt }}=1.6$, which is completely consisitent with the results of the scalar interpolations. This is our optimal convergence criterion for the IRP, since the use of $A B C$ typically introduces error of that order. Figure 4.10 also shows that very modest amounts of additional effort would be required to attain higher levels of accuracy. Thus, the Newton expansion convergence is sufficiently rapid and system independent, that very accurate calculations can be performed with minimal effort in convergence tests.

\section{Order of Time Quadrature}

The convergence of the reaction probability $P_{v=0, j=4}(E=0.9 \mathrm{eV})$ is examined with respect to the order $N_{\text {quad }}$ of the time quadrature. The solid line uses GaussLegendre quadrature, the squared line uses the trapezoidal rule, and the dashed line uses Gauss-Chebyshev quadrature (of the first kind). The Gauss-Legendre quadrature is clearly the most efficient, attaining machine accuracy with $N_{\text {quad }}=28$ points. The trapezoidal rule shows second order convergence, i.e. the slope of the $\log$ (relative error) vs. $\log \left(N_{\text {quad }}\right)$ plot is $\cong-2$. The Gauss-Chebyshev quadrature demonstrates second order convergence as well. This indicates that the integrand does not resemble the weight function in Gauss-Chebyshev quadrature, $w(x)=\left(1-x^{2}\right)^{-1 / 2}$. We use $N_{\text {quad }}=40$ for all subsequent scattering calculations to ensure that exact integration is obtained (provided that $t_{s}=50$ ). We note that Gauss-Legendre quadrature with 40 points exactly integrates a polynomial of degree $\sim 80$, which is precisely the Newton polynomial used.

\section{Total Propagation Time}

Thus far we have shown how to control the error introduced by the time dependent solution of the $\mathrm{ABC}$ system. The remaining source of error comes from 


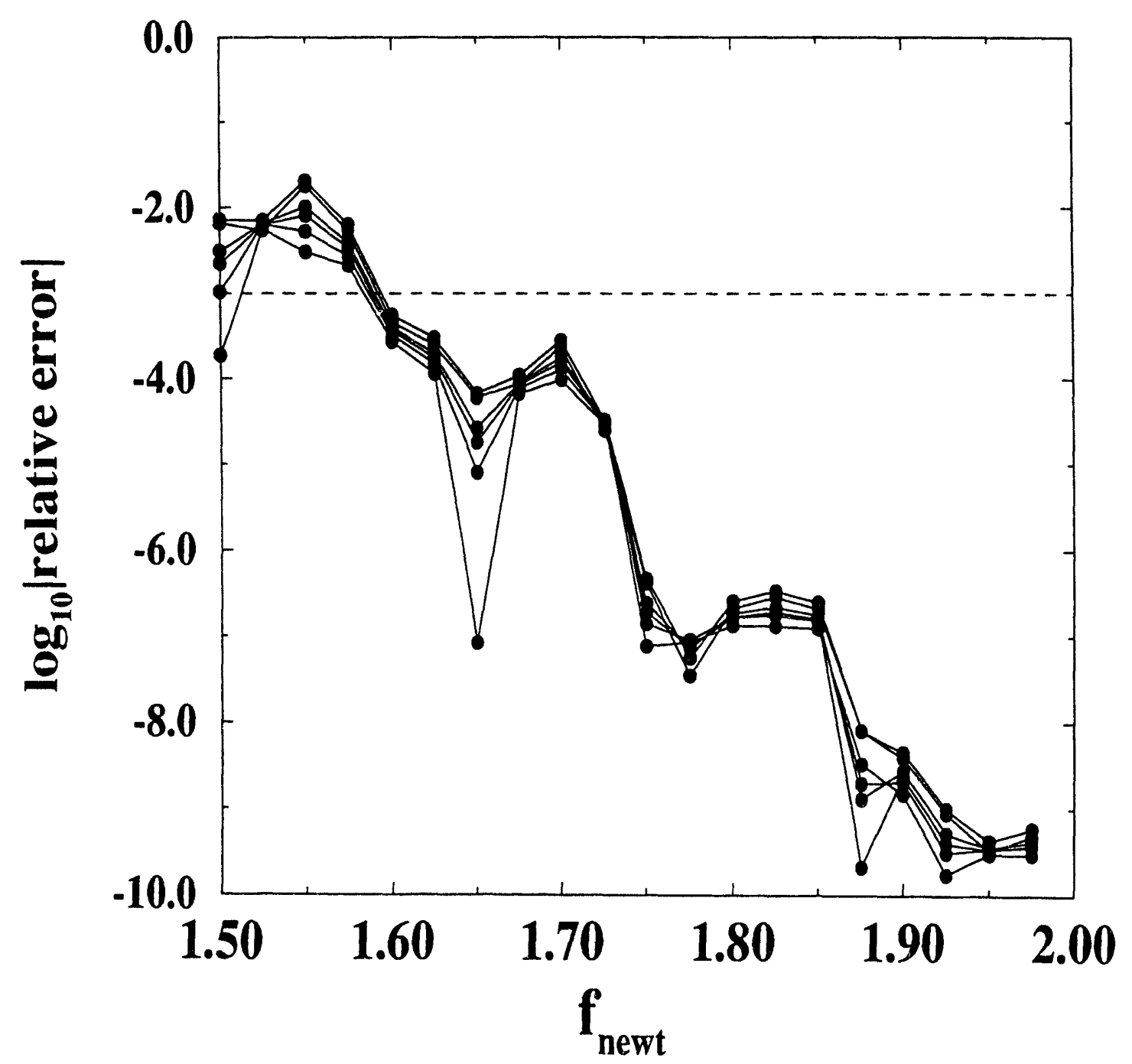

Figure 4.10: Convergence of initial state reaction probabilities for various energies and initial states of the $\mathrm{D}+\mathrm{H}_{2}(v, j)$ system with respect to the Newton polynomial convergence parameter $f_{\text {newt }}$. The systems studied are $[E=0.9 \mathrm{eV}(v, j)=(0,0),(0,4)]$. $[E=1.0 \mathrm{eV}(v, j)=(0,6),(1,4)]$ and $[E=1.1 \mathrm{eV}(v, j)=(0,2),(1,2)]$. Rapid convergence is seen for all systems studied. Three digit accuracy can be obtained reliably using $f_{\text {newt }}=1.6$, consistent with the results of scalar interpolations. Higher levels of accuracy can be obtained with very modest amounts of additional effort. 


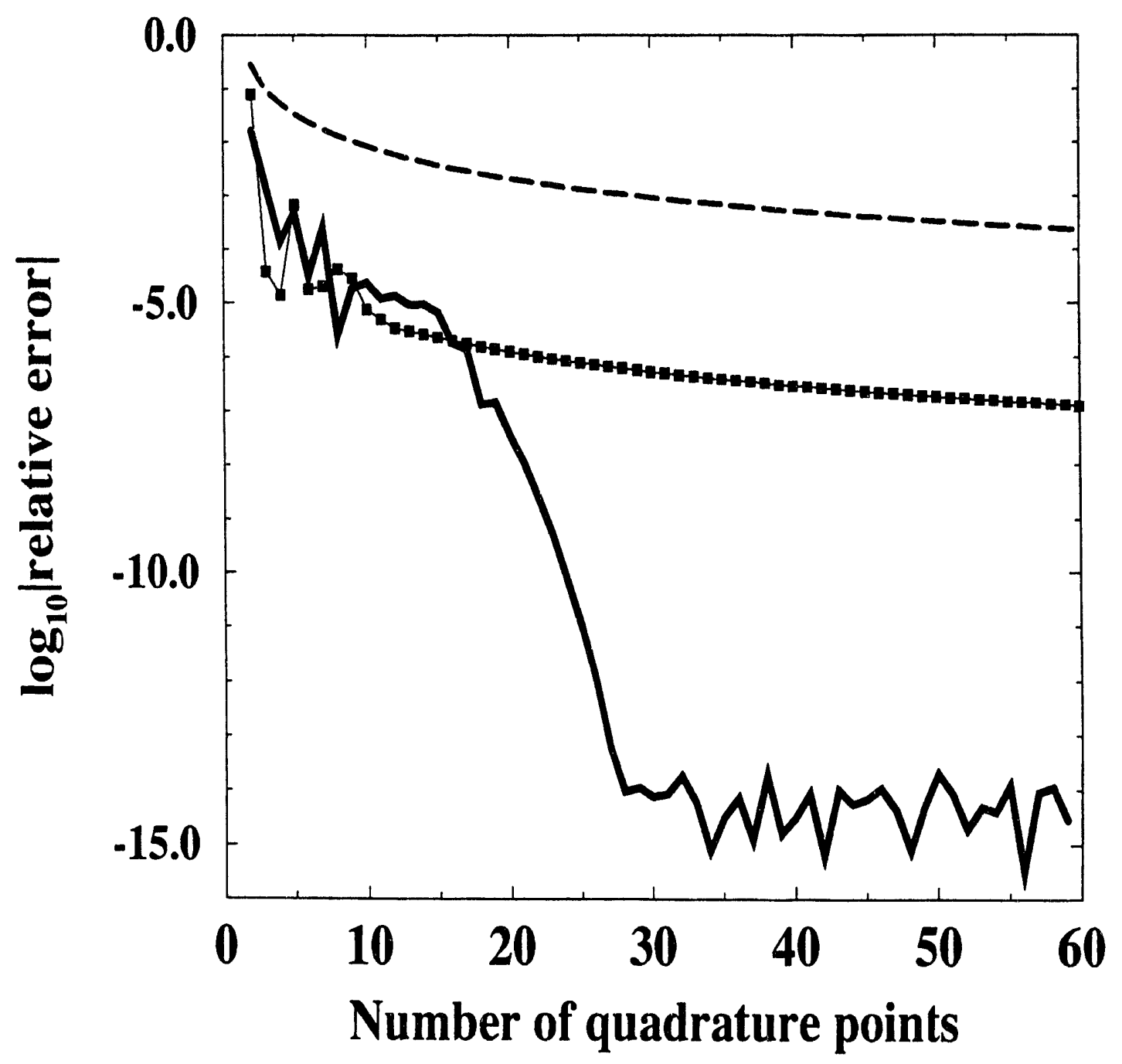

Figure 4.11: Convergence of the initial state reaction probability $P_{v=0, j=4}(E=0.9 \mathrm{eV})$ for the $\mathrm{D}+\mathrm{H}_{2}(v, j)$ system with respect to the order $N_{\text {quad }}$ of the time quadrature. The solid line uses Gauss-Legendre quadrature, the squared line uses the trapezoidal rule, and the dashed line uses Gauss-Chebyshev quadrature (of the first kind). The Gauss-Legendre quadrature is clearly the most efficient, attaining machine accuracy with $N_{\text {quad }}=28$ points. For all subsequent scattering calculations, we use $N_{\text {quad }}=40$. This choice for $N_{\text {quad }}$ is consistent with exact Gaussian integration of an $80^{\text {th }}$ degree Newton polynomial. 
using a finite total propagation time $T_{A B C}$. We control this error by monitoring the residual $r_{N}$ defined in Eq. (4.58), and stopping the calculation when $r_{N}<\delta$, an input convergence parameter. We expect to see exponential decay of the integrand via $r_{N}=e^{-\Gamma(N+1) \Delta t / \hbar}$, which defines an effective absorption rate $\Gamma$. By definition, then, we have $\delta=e^{-\Gamma T_{A B C} / \hbar}$. Therefore, the total propagation time satisfies $T_{A B C} \propto$ $-\log _{10} \delta$. Although specifying either $T_{A B C}$ or $\delta$ as the input convergence parameter is formally equivalent, $\delta$ is more convenient. Figure 4.12 demonstrates the convergence, with respect to $-\log _{10} \delta$, of the initial state reaction probabilities studied in Fig. 4.10. The thick solid line is what would occur if $\delta$ perfectly controlled the error in the reaction probabilities. The solid line with squares is the average of the error, and the dashed line with circles is the maximum error from the systems studied; all as a function of $\delta$. We see the remarkable result that, even in the worst case of maximum error, $\delta$ reliably controls the error in the reaction probability. Thus, even if we were studying a system in resonance (i.e. a small effective absorption rate $\Gamma$ ) requiring a larger $T_{A B C}, \delta$ would not change. This kind of control is an important aspect of any numerical method, i.e. that one be able to determine a priori how accurate the calculation is and consequently how much computational effort is required.

\subsection{Concluding Remarks}

We have derived the $\mathrm{ABC}$ formulation of quantum reactive scattering theory, and applied it to the calculation of the initial state selected reaction probability. By exploiting the highly localized nature of forces in reactive scattering, the $\mathrm{ABC}$ formulation facilitates the direct calculation of detailed or averaged reaction probabilities while sampling only a finite region of space.

We have not been able to find a direct polynomial expansion which is accurate, has a fixed and small memory requirement, and is guaranteed to converge the $A B C$ Green's function. We have attributed this to the fact that none of the direct expansions considered above exploit the finite time nature of the reactive collision. By implicitly containing the effects of infinite time dynamics, the direct expansions suffer from numerical instability by attempting to represent a nearly singular function. 


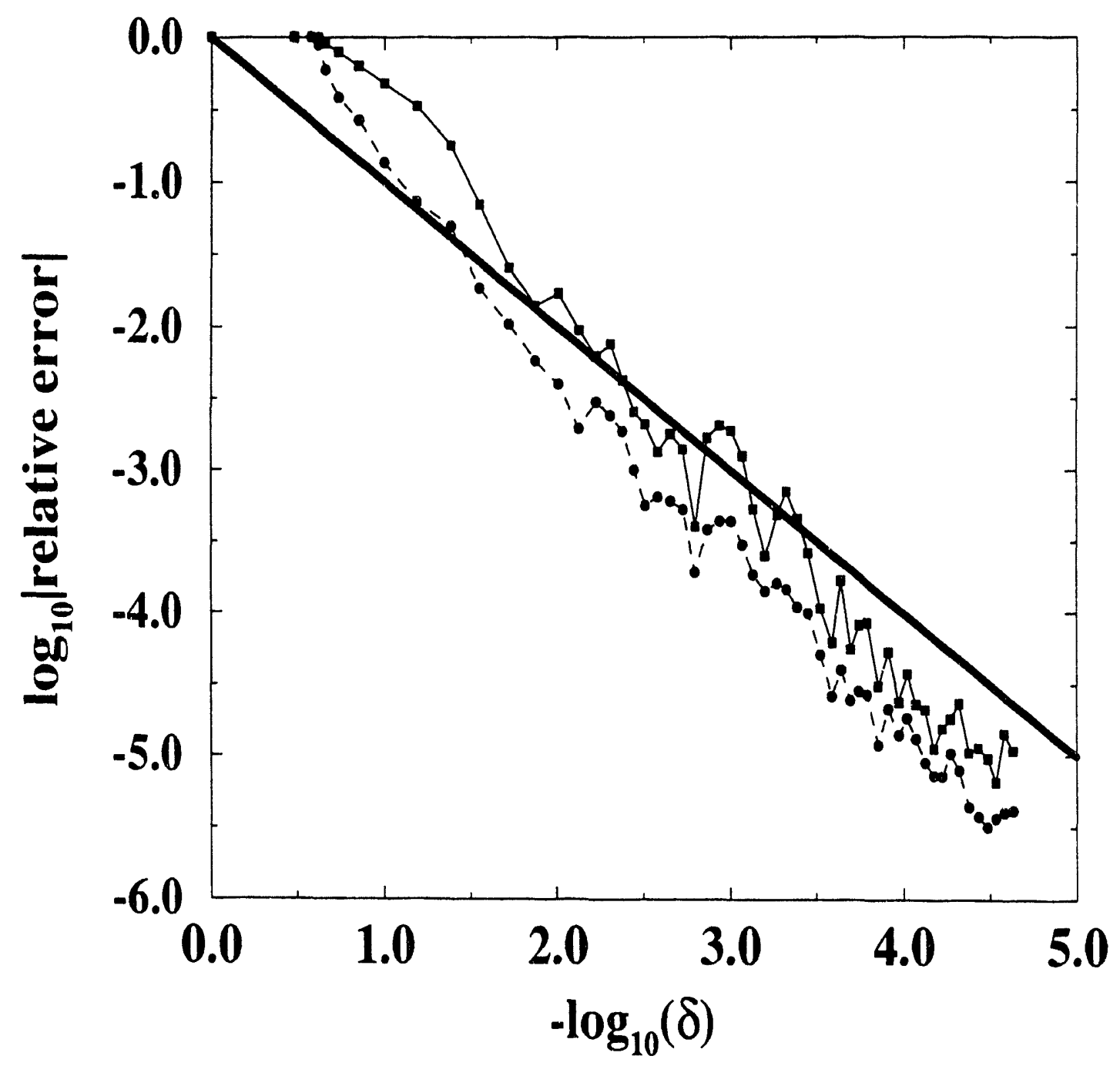

Figure 4.12: Convergence of initial state reaction probabilities for various energies and initial states of the $\mathrm{D}+\mathrm{H}_{2}(v, j)$ system with respect to the total propagation time convergence parameter $\delta$. The systems studied are the same as in Fig. 4.10. The thick solid line is what would occur if $\delta$ perfectly controlled the error in the reaction probabilities. The solid line with squares is the average of the error, and the dashed line with circles is the maximum error from the systems studied; all as a function of $\delta$. We see the remarkable result that, even in the worst case of maximum error, $\delta$ reliably controls the error in the reaction probability. 
We have found that exploiting the time dependent picture of the $A B C$ Green's function leads to a stable and efficient algorithm for solving the ABC system. The new method is based on half Fourier transforming the $A B C$ propagator, which is accurately represented $i$, a Newton polynomial expansion. We have shown that this approach, called the Newton algorithm, is capable of obtaining converged reaction probabilities with very straightforward accuracy control.

The Newton algorithm was applied to calculating initial state selected reaction probabilities for three dimensional $\mathrm{D}+\mathrm{H}_{2}(v, j) \rightarrow \mathrm{DH}+\mathrm{H}$ with zero total angular momentum. We found that the probabilities with initial $j=1$ were the largest, and attributed this effect to a small amount of orbital angular momentum helping to focus the system into more reactive geometries.

Although not stated in the body of this Chapter, we discuss the computational effort required by these reactive scattering calculations. The three dimensional grids contained ca. 4000 points, using no more than 3 Mbytes of core memory. In addition, for each energy and initial reactant state, the entire calculation required $c a$. 35 seconds on an IBM RS 6000/Model 550. The scaling of CPU time from the two dimensional scattering case (collinear reaction-data not shown) to the three dimensional case is very encouraging. Our preliminary findings suggest that the scaling is roughly $N^{1}$. This scaling law is highly system dependent, however. The most extreme case where deviations from this scaling law would arise are systems with eigenvalues close to the real axis (resonances) in which the CPU time would be roughly proportional to the inverse of the imaginary part of the resonant eigenvalue. In the case of $\mathrm{D}+\mathrm{H}_{2}$, where a broad resonance has been observed at $E \cong 0.95 \mathrm{eV}$ [48], we a find negligible effect on the CPU time. As such, in cases where very sharp resonances are not important, this gentle scaling law should hold.

To speculate on the requirements for larger systems, we recall that the operation count for the Newton algorithm is $N_{i t e r}(n F+1) N$, where

$$
\begin{aligned}
N_{\text {iter }} & =\left(T_{A B C} / \Delta t\right) K_{\text {opt }}\left(t_{s}\right) \\
& =T_{A B C} \Delta \mathbf{Z} / \hbar \times f_{\text {newt }} / 2 \\
& \cong T_{A B C} \Delta \mathbf{Z} / \hbar
\end{aligned}
$$


This result is ubiquitous in time dependent studies, and is closely related to the condition number of the $A B C$ system. To estimate the $C P U$ time required to perform a six dimensional (6D) reactive scattering calculation with $10^{6}$ grid points, we assume that $T_{A B C}, n$, and $f_{\text {newt }}$ are unchanged in the $6 \mathrm{D}$ system. Furthermore, assuming that the spectral range in the $6 \mathrm{D}$ system is doubled from the $3 \mathrm{D}$ case, the CPU time is roughly 10 hours on the IBM RS 6000/Model 550. Thus, with the present assumptions, the $6 \mathrm{D}$ calculation is now feasible.

We discuss the relationship between the time dependent solution to the $A B C$ system and conventional wavepacket propagation methods. There are two essential differences. First, in the $A B C$ formulation the starting vector is energy dependent, whereas typical wavepacket calculations use an energy independent starting vector. Second, we perform the Fourier transform at the beginning of the propagation, whereas most wavepacket calculations transform after propagation. Our method is expected to perform most efficiently for direct reactions, i.e. those which do not involve complex formation.

In the next Chapter, we apply the Newton algorithm to the calculation of numerically exact cross sections and rate constants for the vibrationally excited reaction $\mathrm{D}+\mathrm{H}_{2}(v=1, j) \rightarrow \mathrm{DH}+\mathrm{H}$. We show that the $\mathrm{ABC}$ Newton method is the most direct route to date for accurate reaction cross sections.

\section{References}

[1] D. Kliner, D. E. Adelman, and R. N. Zare, J. Chem. Phys. 94, 1069 (1991).

[2] M. J. Bronikowski, W. R. Simpson, B. Girard, and R. N. Zare, J. Chem. Phys. 95, 8647 (1991).

[3] L. S. Rodberg and R. M. Thaler, Introduction to the Quantum Theory of Scattering (Academic, New York, 1967).

[4] W. H. Thompson and W. H. Miller, Chem. Phys. Lett. 206, (1993) 123.

[5] H. Tal-Ezer and R. Kosloff, J. Chem. Phys. 81, (1984) 3967. 
[6] R. Kosloff, J. Phys. Chem. 92, 2087 (1988).

[7] A brief summary of the Newton algorithm appeared previously in the following article:

S. M. Auerbach and C. Leforestier, Comput. Phys. Commun. 00, 0000 (1993).

[8] D. Neuhauser and M. Baer, J. Chem. Phys. 91, (1989) 4651.

[9] W. H. Miller, J. Chem. Phys. 61, (1974) 1823

[10] D. Neuhauser, R. S. Judson, D. J. Kouri, D. A. Adelman, N. E. Shafer, D. A. V. Kliner, and R. N. Zare, Science 257, 519 (1992).

[11] G. C. Groenenboom and D. T. Colbert, J. Chem. Phys. 99, 0000 (1993).

[12] For a recent review, see:

W. H. Miller, Annu. Rev. Phys. Chem. 41, 245 (1990).

[13] T. Seideman and W. H. Miller, J. Chem. Phys. 96, 4412 (1992); 972499 (1992).

[14] W. H. Miller, S. D. Schwartz, and J. W. Tromp, J. Chem. Phys. 79, (1983) 4889.

[15] W. H. Press, B. P. Flannery, S. A. Teukolsky, and W. T. Vetterling, Numerical Recipes: The Art of Scientific Computing (Cambridge University, Cambridge, 1986).

[16] J. Z. H. Zhang, J. Chem. Phys. 94, 6047 (1991).

[17] C. Leforestier and W. H. Miller, J. Chem. Phys., submitted.

[18] R. A. Friesner, J. A. Bentley, M. Menou, and C. Leforestier, J. Chem. Phys. 99, 324 (1993).

[19] D. O. Harris, G. G. Engerholm, and W. D. Gwinn, J. Chem. Phys. 43, (1965) 1515.

[20] A. S. Dickinson and P. R. Certain, J. Chem. Phys. 49, (1968) 4209. 
[21] J. C. Light, I. P. Hamilton, and J. V. Lill, J. Chem. Phys. 82, (1985) 1400.

[22] D. Kosloff and R. Kosloff, J. Comput. Phys. 52, 35 (1983).

[2.3] J. Z. H. Zhang, S.-I. Chu, and W. H. Miller, J. Chem. Phys. 88, (1988) 6233.

[24] B. N. Parlett, The Symmetric Eigenvalue Problem (Prentice-Hall, Englewood Cliffs, NJ, 1980).

[25] G. C. Groenenboom, Report, Netherlands Organization for Scientific Research (NWO), 1992.

[26] C. Lanczos, J. Res. Natl. Bur. Stand. 45, (1950) 255.

[27] C. C. Paige and M. A. Saunders, SIAM J. Numer. Anal. 12, (1975) 617.

[28] J. K. Cullum and R. A. Willoughby, J. Comput. Phys. 44, 329 (1981).

[29] J. J. Jiang and J. S. Hutchinson, J. Chem. Phys. 93, 5643 (1990).

[30] S. Dallwig, N. Fahrer, and C. Schlier, Chem. Phys. Lett. 191, 69 (1992).

[31] J. K. Cullum and R. A. Willoughby, Lanczos Algorithms for Large Symmetric Eigenvalue Computations (Birkhäuser, Boston, 1985), Vol. 1.

[32] Y. Saad and M. H. Schultz, SIAM J. Sci. Stat. Comput. 7, (1986) 856.

[33] J. J. Sakurai, Modern Quantum Mechanics (Addison Wesley, Redwook City, 1985).

[34] D. T. Colbert, private communication.

[35] D. E. Manolopoulos, R. E. Wyatt, and D. C. Clary, J. Chem. Soc. Faraday Trans. 86, 1641 (1990).

[36] M. S. Reeves, D. C. Chatfield, and D. G. Truhlar, J. Chem. Phys. 99, 2739 (1993). 
[37] L. Fox and I. B. Parker, Chebyshev Polynomials in Numerical Analysis (Oxford Unviersity Press, New York, 1968).

[38] The NAG Fortran Library Introductory Guide: Mark 14.

[39] J. Stoer and R. Bulirsch, Introduction to Numerical Analysis (Springer, New York, 1980).

[40] M. Berman, R. Kosloff, and H. Tal-Ezer, J. Phys. A 25, 1283 (1992).

[41] M. Abramowitz and I. A. Stegun, Handbook of Mathematical Functions with Formulas, Graphs, and Mathematical Tables, (Wiley, New York, 1972).

[42] R. V. Churchill and J. W. Brown, Complex Variables and Applications (McGrawHill, New York, 1990).

[43] R. Kosloff in Time Depedent Quantum Molecular Dynamics, ed. by J. Broeckhove and L. Lathouwers, NATO ASI Series B: Phys. vol. 299 (Plenum, New York, 1992), pp. 97-116.

[44] H. Tal-Ezer, R. Kosloff, and C. Cerjan, J. Comput. Phys. 100, 179 (1992).

[45] C. Leforestier, R. H. Bisseling, C. Cerjan, M. D. Feit, R. Friesner, A. Guldberg, A. Hammerich, G. Jolicard, W. Karrlein, H.-D. Meyer, N. Lipkin, O. Roncero, and R. Kosloff, J. Comput. Phys. 94, 59 (1991).

[46] T. Seideman and W. H. Miller, J. Chem. Phys. 95, 17G8 (1991).

[47] D. Zhang and J. Z. H. Zhang, J. Chem. Phys., submitted.

[48] J. Z. H. Zhang and W. H. Miller, J. Chem. Phys. 91, (1989) 1528. 


\section{Chapter 5}

\section{The $\mathbf{D}+\mathbf{H}_{2}(v=1)$ Rate Constant}

\subsection{Introduction}

In the previous Chapter, we developed a theoretical formalism using absorbing boundary conditions $(A B C)$ to compute efficiently the initial state selected reaction probability (IRP). The $A B C$ formulation derives its efficiency from focusing on the highly localized region of space where reactive forces are important. We described a numerical solution of the ABC system, called the Newton algorithm, which is ideal in many ways. First, it only requires locating the region in energy space where the eigenvalues of the ABC system are likely to be. Second, once the proper spectral range is determined, the Newton algorithm gives rapid convergence with automated accuracy control. Finally, the Newton algorithm has a fixed and small memory requirement, making it applicable for larger chemical systems. The Newton algorithm derives its efficiency from exploiting the time dependent picture of the ABC Green's function, which removes problematic poles encountered in other solution algorithms. In the present Chapter, we apply the ABC Newton method to demonstrate its computational efficiency. As we will show, we are able to converge initial state selected reaction cross sections in as little CPU time as 10 minutes on an IBM RS/6000.

An important and non-trivial application of the ABC initial state selected formalism is the calculation of the $\mathrm{D}+\mathrm{H}_{2}(v=1) \rightarrow \mathrm{DH}+\mathrm{H}$ rate constant. This quantity has received much attention in an attempt to resolve a fairly large discrepancy. 
between experiment [1-4] and theory [5-12] with the experimental results typically being one or two orders of magnitude larger than the theoretical ones. Surprisingly, the experimental rate constants varied much more from group to group than did the theoretical ones. For the following discussion, we report the rate constant $k_{v=1}(T)$ in units of $10^{-13} \mathrm{~cm}^{3}$ molecule $\mathrm{ec}^{-1} \mathrm{se}^{-1}$.

Early experimental measurements of this rate constant were obtained by determining the concentration of the product $\mathrm{H}$ atoms with electron paramagnetic resonance (EPR). The first published experimental value, measured by Keuba et al. [1] in 1979 is $k_{v=1}(T=300 K)=120 \pm 50$. This value is reasonable for an activationless reaction, and thus generated excitement regarding the efficiency of vibrational excitation in promoting chemical reaction. This experiment was followed by two theoretical calculations, a distorted wave Born approximation (DWBA) by Sun et al. [5] in 1980 yielding $k_{v=1}(T=300 \mathrm{~K})=5.0$, and a quasiclassical trajectory calculation (QCT) by Mayne and Toennies [6] in 1981 giving $k_{v=1}(T=300 K)=1.7$. From previous tests. it is known that the DWBA is accurate in the threshold energy region but overestimates reaction probabilities for higher energies, and thus overestimates the rate constant. Also, since the QCT calculation lacks the contribution from tunneling, it underestimates the exact rate constant. In principle, then, the two theoretical results bracketed the exact rate constant for the potential energy surface (PES) used in these calculations. The $a b$ initio surface was computed by Siegbahn and Liu [13] and fitted by Truhlar and Horowitz $[14,15]$. The Liu-Siegbahn-Truhlar-Horowitz (LSTH) PES is the most accurately known PES for a neutral molecular reactive scattering system. Thus, this discrepancy between experiment and theory is quite significant.

Glass and Chaturvedi [2] performed a measurement in 1982 which accounted for one order of magnitude in the discrepancy. As opposed to the Keuba experiment, Glass and Chaturvedi prepared $\mathrm{H}_{2}(v=1)$ without recourse to vibrationally excited $\mathrm{HF}^{*}$. Using vibrationally excited $\mathrm{HF}^{*}$ was thought to contribute indirectly to the detected population of products through the following mechanism:

$$
\begin{aligned}
& \mathrm{D}+\mathrm{HF}^{*} \rightarrow \mathrm{HD}+\mathrm{F} \\
& \mathrm{F}+\mathrm{H}_{2} \rightarrow \mathrm{HF}+\mathrm{H} .
\end{aligned}
$$

EPR detection of the $\mathrm{H}$ atoms produced by the reactions above gives a rate con- 
stant which is too large. Indeed, by avoiding this source of contamination, Glass and Chaturvedi obtained $k_{v=1}(T=297 K)=9.8 \pm 3.0$. A similar experiment was performed by Rozenshtegn et al. [3] in 1984 yielding $k_{v=1}(T=297 K)=16.1 \pm 4.9$. Since the error bars of these two measurements overlap, this order of magnitude for $k_{v=1}(T=300 K)$ was thought to be correct. A variety of calculations were performed between 1983 and 1985, yielding results between 0.9 and 2.9 (a prediction of 10.0 was made by Pollack et al. [10] which was based on some theoretically ill-defined "reinterpretations.") Thus, a smaller but persistent discrepancy remained.

Dreier and Wolfrum [4] accounted for roughly another order of magnitude by using coherent anti-Stokes Raman scattering (CARS) spectroscopy to monitor directly most of the reagents in the system. They obtained $k_{v=1}(T=310 K)=1.9$ \pm 0.2 . This measurement should be more reliable than the previous ones because of the direct observation of all reagent concentrations. We speculate why the previous measurements gave larger values for the rate constant. The Glass and Rozenshregn experiments both involved a great excess of $\mathrm{D}$ atoms over $\mathrm{H}_{2}(v=1)$ molecules. These experiments might have detected $\mathrm{H}$ atom contamination from the reaction $\mathrm{D}+\mathrm{HD} \rightarrow$ $\mathrm{D}_{2}+\mathrm{H}$, where $\mathrm{HD}$ is the nascent product from the $\mathrm{D}+\mathrm{H}_{2}$ under study. Thus, the Glass and Rozenshtegn experiments might not have detected nascent populations. Based on the direct and thorough nature of the Dreier measurement, we take their result for comparison.

The most accurate theoretical treatment of this rate constant is by Zhang and Miller [16] in 1989, using the $S$-matrix version of the Kohn variational principle. Their result is $k_{y=1, j=0}(T=300 K)=1.63$. However, they reported the rate constant for $\mathrm{D}+\mathrm{H}_{2}(v=1, j=0)$, and the experiment by Dreier and Wolfrum involved a thermal distribution of reactant $j$-states. With the extreme importance of fundamentally understanding the role of vibrational excitation in chemical reactions, we undertook the calculation of this rate constant with the present initial state selected formalism. We will show that quantitative agreement has now been obtained [17].

As important as exact reactive scattering calculations may be, approximations are indispensable in developing useful tools for estimating the reactivity of complex systems. An important model is the $J$-shifting approximation [18-20] (JSA), 
which allows one to estimate observables such as cross sections and rate constants when only $J=0$ calculations are possible. It does so by ignoring the Coriolis coupling, and by assuming that the centrifugal coupling is only important near the transition state geometry. In this way, a vibration rotation decoupling approximation is made, which allows the total energy to be partitioned into a contribution from the $J=0$ Hamiltonian (the "vibrational" part), and a contribution from the overall rotational Hamiltonian.

The accuracy of the JSA was tested by Bowman [18], who examined the $J=4$ partial cumulative reaction probability for $\mathrm{H}+\mathrm{H}_{2}$. He found excellent agreement with the exact results of Chatfield et al. [21] up to total energy $E \cong 1.2 \mathrm{eV}$. In addition, Takada et al. [22] used the JSA to compute cross sections and rate constants for $\mathrm{D}+\mathrm{H}_{2}(v=j=0)$. Comparing to the exact results of Zhang and Miller [16], they too found excellent agreement for low enough energy. However, the JSA has never been tested in the important case of an initial state selected reaction with rovibrationally excited reactants. This is particularly significant in developing estimates of reaction rates to compare with the state and bond selected experiments of Crim and co-workers [23], and Zare and co-workers [24]. In the present study, we test the JSA in the calculation of $\mathrm{D}+\mathrm{H}_{2}(v=1, j)$ rate constants. We will show that the JSA is qualitatively correct when selecting individual $j$-states, and is semi-quantitative once the rate constants are thermally averaged over the $j$-states.

\subsection{General Methodology}

We now discuss the formalism used in the present Chapter to obtain the initial state selected rate constant for an atom-diatom reaction. We briefly review the general rate constant formulae and the ABC method of obtaining reaction probabilities. The Newton algorithm for the ABC Green's function was thoroughly discussed in the previous Chapter. 


\subsubsection{General Rate Constant Formulae}

The quantity of experimental interest is the thermal rate constant with initial vibrational state selection, $k_{v}(T)$. This corresponds to a rate measurement of the total yield of a reaction where all the motions of the reactants are in thermal equilibrium at temperature $T$, except for the diatomic vibration. The latter is promoted to a non-equilibrium state by laser excitation. This rate constant can be obtained from averaging the more detailed $(v, j)$-selected rate constant via

$$
k_{v}(T)=\sum_{j=0}^{\infty} p_{v, j}(T) k_{v, j}(T)
$$

where

$$
p_{v, j}(T)=\frac{(2 j+1) w_{j} e^{-\varepsilon_{v, j} / k_{B} T}}{\sum_{j^{\prime}=0}^{\infty}\left(2 j^{\prime}+1\right) w_{j^{\prime}} e^{-\varepsilon_{v, j^{\prime}} / k_{B} T}}
$$

is the rotational distribution of reactant diatomics in vibrational state $v$. In Eq. (5.2), $\varepsilon_{v, j}$ is the reactant diatomic rovibrational energy, $k_{B}$ is Boltzmann's constant, and $w_{j}$ accounts for any symmetry statistics of the reactant diatomic (e.g. for $\mathrm{H}_{2}, w_{j}=1$ for even $j$, and 3 for odd $j$ ). We note that there is also an average over $K$, the projection quantum number of $j$, which is discussed below.

The reaction of a diatomic molecule in state $(v, j)$ with an atom approaching with a distribution of velocities has a rate constant given by [25]

$$
k_{v, j} \equiv\left\langle v_{t} \sigma_{v, j}\left(v_{t}\right)\right\rangle
$$

where $v_{t}$ is the translational velocity, $\sigma_{v, j}\left(v_{t}\right)$ is the initial state selected reaction cross section, and $\langle\cdots\rangle$ denotes an average over the velocity distribution. If the velocity average is determined by Maxwell-Boltzmann distribution, the rate constant is labelled by the temperature of the distribution, and is given by [25]

$$
k_{v, j}(T)=\sqrt{\frac{8 k_{B} T}{\pi \mu_{t}}}\left(k_{B} T\right)^{-2} \int_{0}^{\infty} d E_{t} E_{t} e^{-E_{t} / k_{B} T} \sigma_{v, j}\left(E_{t}\right)
$$

where $E_{t}=\mu_{t} v_{t}^{2} / 2$ is the initial translational energy of the reactants, and $\mu_{t}$ is the translational reduced mass. The initial state selected reaction cross section can be 
obtained from quantum mechanical reaction probabilities by partial wave expansion [25], in which

$$
\sigma_{v, j}\left(E_{t}\right)=\frac{\pi}{k_{t}^{2}} \sum_{J=0}^{\infty} \frac{2 J+1}{2 j+1} \sum_{l=|J-j|}^{J+j} P_{v, j, l}^{J}\left(E_{t}\right),
$$

where $k_{t}$ is the translational wave vector associated with $E_{t}$ and $\mu_{t}$. We perform the average over $K$ by averaging the space-fixed (SF) reaction probabilities over $l$, the orbital angular momentum quantum number. In Eq. (5.5), $P_{v, j, l}^{J}\left(E_{t}\right)$ is the initial state selected reaction probability defined by

$$
P_{v, j, l}^{J}\left(E_{t}\right) \equiv \sum_{\left\{v^{\prime}, j^{\prime}, l^{\prime}\right\}} P_{v^{\prime}, j^{\prime}, l^{\prime} \leftarrow v, j, l}^{J}(E)
$$

where $\left\{v^{\prime}, j^{\prime}, l^{\prime}\right\}$ is the open channel space of products at total energy $E=E_{t}+\varepsilon_{v, j}$, and $P_{v^{\prime}, j^{\prime}, l^{\prime} \leftarrow v, j, l}^{J}(E)$ are the state-to-state reaction probabilities. The reaction probability in Eq. (5.6) is the fundamental quantity of interest which we obtain with the $A B C$ formalism reviewed below.

\subsubsection{ABC Formulation of Quantum Reactive Scattering}

The $A B C$ approach to quantum reactive scattering was originally derived to compute the cumulative reaction probability $[26,27]$. It was then applied to the calculation of initial state selected and state-to-state reaction probabilities [28]. Thorough discussions of the theory can be found in these references. For completeness, a brief outline of the formulae relevant for atom-diatom reactions is provided below.

We use $A B C$ to achieve two related goals [29-32]. First, by absorbing all outgoing flux the scattering problem is converted into an effective non-Hermitian bound state problem, in which standard $L^{2}$ basis set techniques may be used. Second, by placing the absorbing potentials very close to the interaction region, some $[29,28]$ or all [26] of the asymptotic state information can be implicitly averaged, facilitating more economical calculations. In this spirit, the $A B C$ initial state selected reaction probability is given by [28]

$$
P_{v, j, l}^{J}\left(E_{t}\right)=\frac{2}{\hbar}\left\langle\Psi_{v, j, l}^{J M}\left(E_{t}\right)\left|\hat{\epsilon}_{p}\right| \Psi_{v, j, l}^{J M}\left(E_{t}\right)\right\rangle
$$


where $\hat{\epsilon}_{p}$ is the absorbing potential operator in the product region of configuration space. In Eq. (5.7), $\left|\Psi_{v, j,}^{J M}\left(E_{t}\right)\right\rangle$ is the $\mathrm{ABC}$ scattering wavefunction defined by:

$$
\left|\Psi_{v, j, l}^{J M}\left(E_{t}\right)\right\rangle \equiv \hat{G}^{A B C}(E) i \hat{\epsilon}\left|\Phi_{v, j, l}^{J M}\left(E_{t}\right)\right\rangle
$$

where the ABC Green's function is given by

$$
\hat{G}^{A B C}(E)=(E+i \hat{\epsilon}-\hat{H})^{-1} .
$$

In Eq. (5.8), $\hat{\epsilon}$ is the absorbing potential operator for all chemical arrangements and $\left|\Phi_{v, j, l}^{J M}\left(E_{t}\right)\right\rangle$ is a refereuce scattering state with incoming-wave boundary conditions in channel $\left(v, j, l, J, M, E_{t}\right)$. The reaction probabilities are independent of the quantum number $M$, to be defined below. In what follows, we omit the "ABC" superscript with the understanding that we are using the $\mathrm{ABC}$ formulation.

We note that use of an absorbing potential in Eq. (5.9) in the definition of the Green's function is tantamount to replacing the infinitesimal energy $\epsilon$ that arises in formal scattering theory with a coordinate dependent function $\epsilon(q)$. This replacement is valid as long as $\epsilon(q)$ is negligible in the strong chemical interaction region, and absorbs all flux by the edge of the $L^{2}$ basis.

\subsection{Defining the Linear System}

We define the precise linear system to be solved for the $\mathrm{D}+\mathrm{H}_{2}$ quantum reactive scattering calculations. This entails the choice of system coordinates, basis set, asymptotic state, and absorbing potential. We note that, with respect to the coordinates and basis set, much of our work parallels that of Choi and Light [33] in their calculations on the $\mathrm{Ar}-\mathrm{HCl}$ van der Waals complex.

\subsubsection{The Coordinates}

We use the mass-scaled (MS) body-fixed (BF) center of mass Jacobi coordinates of the reactant $\mathrm{D}+\mathrm{H}_{2}$ to define the differential Hamiltonian operator. The two Jacobi vectors are $\mathbf{R}$ for the scattering coordinate, and $\mathbf{r}$ for the vibrational coordinate. The internal coordinates are $\mathbf{q}=(r, R, \gamma)$ where $r=|\mathbf{r}|$ is the MS bond length 
of $\mathrm{H}_{2}, R=|\mathbf{R}|$ is the MS scattering coordinate, and $\gamma=\cos ^{-1}[\mathbf{r} \cdot \mathbf{R} / r R]$ is the bending angle. This choice seems reasonable because the initial state selection requires that more grid points be placed in the reactant region. Also, the use of body-fixed coordinates allows for more economical exact calculations (vide infra) [34, 35]. The coordinates which define the plane of the three particles are the Euler angles to be defined below. Figure 5.1 demonstrates the lab-fixed (LAB), SF and BF coordinate frames. The six dimensional Hamiltonian operator in the center of mass translational frame and body-fixed rotational frame is given by

$$
\hat{H}(\hat{\mathbf{J}}, r, R, \gamma)=\hat{T}^{r}+\hat{T}^{R}+\frac{(\hat{\mathbf{J}}-\hat{\mathbf{j}})^{2}}{2 \mu R^{2}}+\frac{\hat{j}^{2}}{2 \mu r^{2}}+\hat{V}(r, R, \gamma),
$$

where $\hat{\mathbf{J}}$ is the total system angular momentum vector operator, $\hat{\mathbf{j}}$ is the diatomic molecule angular momentum vector operator, and the system mass is

$$
\mu=\left(\frac{M_{D} M_{H} M_{H}}{M_{D}+M_{H}+M_{H}}\right)^{1 / 2}=1298.796 a u .
$$

In Eq. (5.10), the following quantities are:

$$
\begin{aligned}
& \hat{T}^{r}=-\frac{\hbar^{2}}{2 \mu} \frac{\partial^{2}}{\partial r^{2}} \\
& \hat{T}^{R}=-\frac{\hbar^{2}}{2 \mu} \frac{\partial^{2}}{\partial R^{2}},
\end{aligned}
$$

and $\hat{V}(r, R, \gamma)$ is the LSTH PES.

We can use the isotropy of field free space to reduce this six dimensional Hamiltonian to a set of four dimensional ones. The three dimensional vector operator $\hat{\mathbf{J}}$ yields three quantum numbers from quantizing the square modulus $\hat{J}^{2}$, the spacefixed z-axis projection $\hat{J}_{Z_{S F}}$, and the body-fixed z-axis projection $\hat{J}_{Z_{B F}}$. We define $|J M K\rangle$ as the simultaneous eigenstate of these three operators, satisfying

$$
\begin{aligned}
& \hat{J}^{2}|J M K\rangle=\hbar^{2} J(J+1)|J M K\rangle \\
& \hat{J}_{Z_{S F}}|J M K\rangle=\hbar M|J M K\rangle \\
& \hat{J}_{Z_{B F}}|J M K\rangle=\hbar K|J M K\rangle \text {. }
\end{aligned}
$$




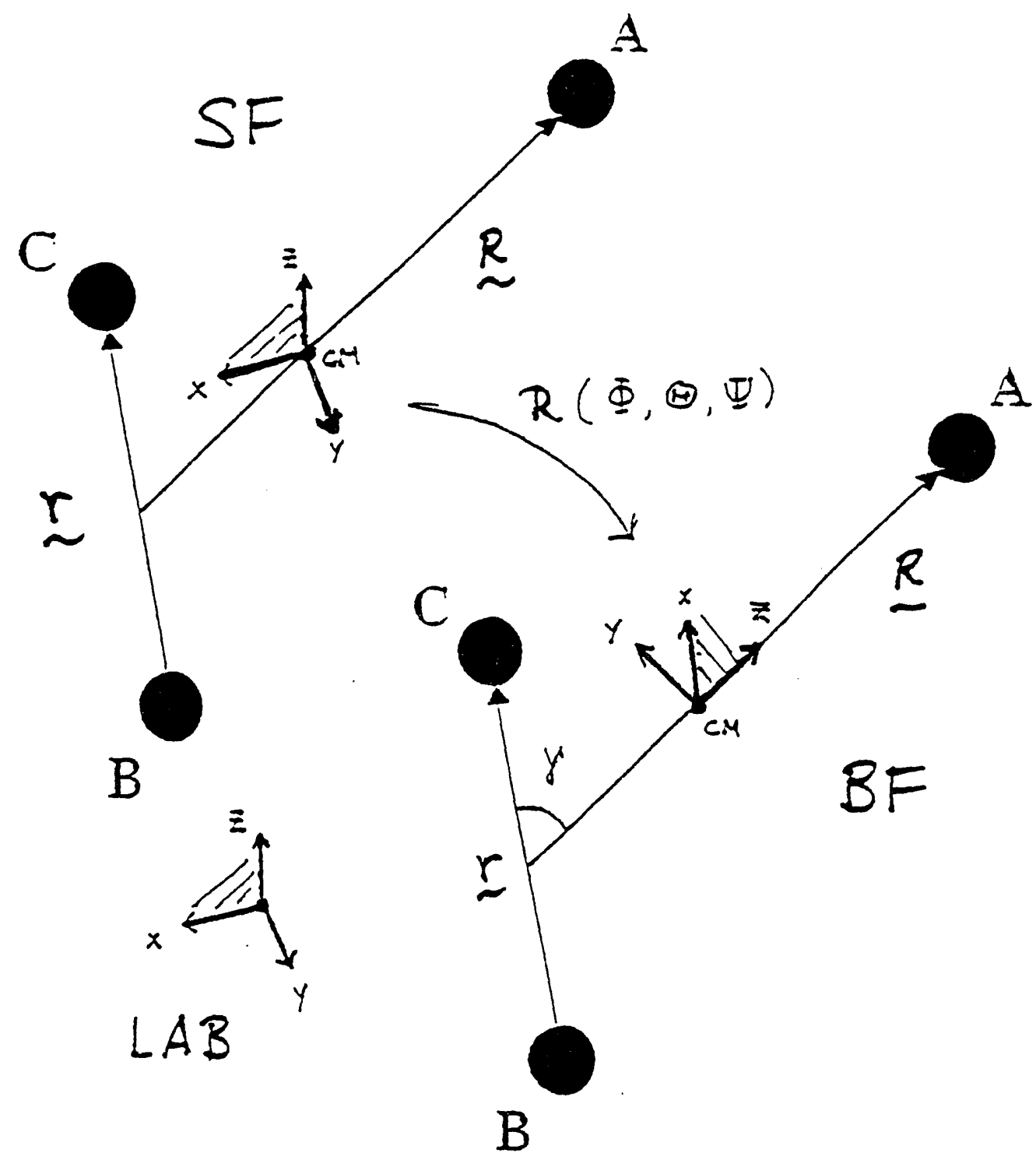

Figure 5.1: The laboratory (LAB) frame, space-fixed (SF) frame, and body-fixed (BF) frame for the $A+B C$ system in Jacobi coordinates. All are right handed axis systems. The SF frame originates on the center of mass (CM) of the $A+B C$ system, and is parallel with the LAB frame, which originates on the experimental apparatus. The BF frame also originates on the center of mass of the $A+B C$ system, but rotates in space with the system so that the BF z-axis lies on the Jacobi scattering coordinate $\mathbf{R}$, and the BF $\mathrm{x}$-axis lies in the plane of the three particles. The transformation from the $\mathrm{SF}$ frame to the $\mathrm{BF}$ frame is a three dimensional rotation symbolized by $\mathcal{R}$, which may be specified in terms of the Euler angles $(\Phi, \Theta, \Psi)$. [The two versions of $A+B C$ in this Figure are identical in every way. The axis systems, however, are different in the two pictures.] The details and conventions are discussed in the main text. 
The position representation of $|J M K\rangle$ using Euler angles is related to the Wigner rotation matrix element (sometimes called the element of the irreducible rotation group). For completeness, we describe our convention for the Euler angles, and for the three dimensional rotation operator. The Euler angles are $(\Phi, \Theta, \Psi)$, which define a three dimensional rotation from a space-fixed axis system $\left(X_{S F}, Y_{S F}, Z_{S F}\right)$ to a body-fixed axis system $\left(X_{B F}, Y_{B F}, Z_{B F}\right)$ which rotates with the molecular system. The first angle $\Phi$ gives a counterclockwise rotation about the $Z_{S F}$ axis, giving an intermediate axis system $\left(X^{\prime}, Y^{\prime}, Z_{S F}\right)$. The second angle $\Theta$ gives a counterclockwise rotation about the $Y^{\prime}$ axis, giving a second intermediate axis system $\left(X^{\prime \prime}, Y^{\prime}, Z_{B F}\right)$ The third angle $\Psi$ gives a counterclockwise rotation about the $Z_{B F}$ axis, giving the final body-fixed axis system. The complete three dimensional rotation is effected by the quantum mechanical rotation operator $\hat{\mathcal{R}}(\Phi, \Theta, \Psi)$ defined by

$$
\hat{\mathcal{R}}(\Phi, \Theta, \Psi) \equiv \exp \left[-i \Psi \hat{J}_{Z_{B F}} / \hbar\right] \exp \left[-i \Theta \hat{J}_{Y^{\prime}} / \hbar\right] \exp \left[-i \Phi \hat{J}_{Z_{S F}} / \hbar\right]
$$

By representing the rotated angular momenta $\hat{J}_{Z_{B F}}$ and $\hat{J}_{Y^{\prime}}$ as the result of rotational transformations of the original angular momenta in the SF frame, the above expression for $\hat{\mathcal{R}}(\Phi, \Theta, \Psi)$ can be written with the angles in the opposite order, and all rotations referenced to the original axis system, i.e.

$$
\hat{\mathcal{R}}(\Phi, \Theta, \Psi)=\exp \left[-i \Phi \hat{J}_{Z_{S F}} / \hbar\right] \exp \left[-i \Theta \hat{J}_{Y_{S F}} / \hbar\right] \exp \left[-i \Psi \hat{J}_{Z_{S F}} / \hbar\right]
$$

This latter form is more useful for our subsequent discussion. Based on the eigenstates $|J M\rangle$ and $|J K\rangle$ of a linear rigid rotor, our convention for the Wigner rotation matrix element is [36]

$$
D_{M, K}^{J}(\Phi, \Theta, \Psi) \equiv\langle J M|\hat{\mathcal{R}}(\Phi, \Theta, \Psi)| J K\rangle
$$

Many other conventions exist for this matrix element. Our choice is the (we hope) consistent active convention, which is adapted from the text by Rose [36].

We choose to mount the body-fixed z-axis $Z_{B F}$ along the Jacobi scattering coordinate $\mathbf{R}$. In so doing, $(\Phi, \Theta)$ are the azimuthal and polar angles of $\mathbf{R}$, respectively, measured from a SF reference frame. In addition, $\Psi$ is the angle which brings the body-fixed $x$-axis $X_{B F}$ into the plane of the three particles, with a non-negative 
dot product with the Jacobi vibrational vector $\mathbf{r}$. The body-fixed y-axis $Y_{B F}$ orthogonal to the plane of the particles. Furthermore, this choice of body-fixed quantization means that orbital angular momentum has no body-fixed projection. Writing these quantities classically to illustrate the point, we have that $L_{Z_{B F}}=Z_{B F} \cdot(\mathbf{R} \times \mathbf{P})$ $\propto \mathbf{R} \cdot(\mathbf{R} \times \mathbf{P})=0$. As such, the body-fixed projection quantum number for total angular momentum is equal to that for the diatomic angular momentum, since the orbital motion does not contribute along the $Z_{B F}$ direction.

Based on these definitions, we can obtain the four dimensional Hamiltonian from the six dimensional one by integrating out the Euler angles. We first specify the Euler angle representation of $|J M K\rangle$. This is

$$
\tilde{D}_{M, K}^{J}(\Phi, \Theta, \Psi) \equiv\langle\Phi, \Theta, \Psi \mid J M K\rangle=\sqrt{\frac{2 J+1}{8 \pi^{2}}}\left[D_{M, K}^{J}(\Phi, \Theta, \Psi)\right]^{*} .
$$

The prefactor gives the proper normalization for integrating Euler angles over $\int_{0}^{2 \pi} d \Phi$ $\times \int_{-1}^{1} d \cos \Theta \times \int_{0}^{2 \pi} d \Psi$, and the complex conjugate gives the phase convention which reduces to that used by spherical harmonics in the case where either $M$ or $K$ is zero. At this point, we could proceed to integrate out the Euler angles with a basis set of the $\left\{\tilde{D}_{M, K}^{J}(\Phi, \Theta, \Psi)\right\}$. This partially diagonalizes the Hamiltonian, yielding blocks labelled by $J$ and $M=0, \pm 1, \ldots, \pm J$. These blocks depend on $J$, but are indepedent of $M$. Thus, the manifold of $M$ states merely contributes a degeneracy which gives rise to the factor of $2 J+1$ in Eq. (5.5). Each block for a given $J$ and $M$ is a $(2 J+1) \times(2 J+1)$ matrix labelled by $K^{\prime}, K=0, \pm 1, \ldots, \pm J . K$ is not conserved because the axis which defines its projection rotates dynamically in space. We can further block diagonalize the $J M$ block by exploiting the conservation of overall parity, i.e. that the system is invariant to inversion through its center of mass. We do this by integrating out the Euler angles with a parity adapted version of the overall rotation functions. This reduces by roughly half the computational effort required for each reaction probability, as we will show. These can be constructed by analyzing the effect on $\tilde{D}_{M, K}^{J}(\Phi, \Theta, \Psi)$ of the overall parity operator. We leave the details as an exercise to the reader. The parity adapted Wigner functions $B_{M, K}^{J P}(\Phi, \Theta, \Psi)$ with 
parity $P$ are defined by

$$
B_{M, K}^{J P}(\Phi, \Theta, \Psi) \equiv N_{K}\left[\tilde{D}_{M, K}^{J}(\Phi, \Theta, \Psi)+(-1)^{J+K+P} \tilde{D}_{M,-K}^{J}(\Phi, \Theta, \Psi)\right],
$$

where $N_{K}=\left[2\left(1+\delta_{K, 0}\right)\right]^{-1 / 2}$. After integrating out the Euler angles with the parity adapted Wigner functions $\left\{B_{M, K}^{J P}(\Phi, \Theta, \Psi)\right\}$ the Hamiltonian block with parity $P$ becomes $[33,36]$

$$
\begin{aligned}
\hat{H}_{K^{\prime}, K}^{J P}(r, R, \gamma) & =\delta_{K^{\prime}, K}\left\{\hat{T}^{r}+\hat{T}^{R}+\hat{T}^{\gamma}+\frac{\hbar^{2}}{2 \mu R^{2}}\left[J(J+1)-2 K^{2}\right]+\hat{V}(r, R, \gamma)\right\} \\
& -\delta_{K^{\prime}, K+1}\left\{\left(1+\delta_{K, 0}\right)^{1 / 2} \frac{\hbar}{2 \mu R^{2}} \Lambda_{J K}^{+} \hat{j}^{+}\right\} \\
& -\delta_{K^{\prime}, K-1}\left\{\left(1+\delta_{K, 1}\right)^{1 / 2} \frac{\hbar}{2 \mu R^{2}} \Lambda_{J K} \hat{j}^{-}\right\}
\end{aligned}
$$

The inversion symmetry quantum number $P$ determines the range of $K^{\prime}$ and $K$, i.e. when $J+P$ is even $K^{\prime}, K=0, \ldots, J$ and otherwise $K^{\prime}, K=1, \ldots, J$. In Eq. (5.18), the following quantities are

$$
\begin{aligned}
\hat{T}^{\gamma} & =\frac{1}{2 \mu}\left(\frac{1}{r^{2}}+\frac{1}{R^{2}}\right) \hat{j}^{2} \\
\Lambda_{J K}^{ \pm} & =\sqrt{J(J+1)-K(K \pm 1)} .
\end{aligned}
$$

Also, $\hat{j}^{ \pm}$are the usual raising and lowering operators for the diatomic angular momentum in the body-fixed system. The portion which is off diagonal in $K$, the body-fixed projection quantum number, is called the Coriolis coupling with the second line in Eq. (5.18) called the "+" Coriolis coupling the the third line the "-" Coriolis coupling.

We now describe the basis set used to represent the remaining operators in the Hamiltonian. The use of a grid for the internal degrees of freedom and a delocalized basis set for the overall rotation requires that special attention be given to the Coriolis terms. These involve coupling in both the overall rotation and internal bending.

\subsubsection{The Basis Set}

In the present calculations we use a discrete variable representation [37-39] (DVR) for each internal degree of freedom. The DVR gives a diagonal potential 
matrix, and thus, all the coupling is in the one-dimensional kinetic energy matrices. This is a poor, but convenient representation because the multi-dimensional Hamiltonian matrix is sparse, which facilitates iterative calculations based on the sparse matrix-vector multiply $[40,41]$, In practice, we first define a direct prodisct grid in four dimensions, called the primitive grid. This is then truncated based on several criteria to give the final grid used to represent the $A B C$ wavefunction. First, we discuss the construction of the primitive grid, and the relevant kinetic energy matrix elements. Then we discuss the truncation algorithm.

For the two radial coordinates, we use the radial sinc-DVR given by Colbert and Miller [42]. Considering the scattering coordinate first, a grid of $R$ values is defined by $R_{i}=i \Delta R$ where $i=1,2,3, \ldots$. The point at zero is automatically deleted because of the Jacobian weight at the origin. The radial kinetic energy matrix element is

$$
T_{i^{\prime} i}^{R}=\frac{\hbar^{2}}{2 \mu \Delta R^{2}}(-1)^{i^{\prime}-i}\left\{\begin{array}{ll}
\pi^{2} / 3-1 / 2 i^{2}, & i^{\prime}=i \\
\frac{2}{\left(i^{\prime}-i\right)^{2}}-\frac{2}{\left(i^{\prime}+i\right)^{2}}, & i^{\prime} \neq i
\end{array}\right\} .
$$

The same applies for the $r$ coordinate, except with the vibrational grid spacing $\Delta r$. In practice, we have used the same grid spacing for the two radial coordinates, because they are associated with the same mass $\mu$. The grid spacing is chosen by requiring that the number of points per de Broglie wavelength $\left(N_{B}\right)$ is roughly 4 , as was found by Colbert and Miller [42].

For the bending angle, we use an associated Legendre (AL) DVR which properly removes the singularity in the Hamiltonian for collinear geometries when $K>0$. We symmetrize the AL DVR to exploit the exchange symmetry of the two identical $\mathrm{H}$ atoms, allowing us to use half as many angular grid points.

For simplicity, we use a $K$-independent grid $[43,44]$. That is, we obtain grid points $\left\{x_{i}\right\}$ and weights $\left\{w_{i}\right\}$ for the $K=0$ AL functions (i.e. the usual GaussLegendre DVR). We then use these points and weights to construct the angular kinetic energy for all values of $K$ in the Hamiltonian. This is to be contrasted with the treatment of Choi and Light [33] who use different points and weights for each $K$-block. Both approaches are valid, and we wanted to keep the basis set as simple as possible. 
Using $N_{A L}$ symmetrized AL DVR states, and labeling the exchange symmetry blocks by $p=0$ or 1 , the $(p, K)$-dependent angular kinetic energy matrix elements are given by

$$
j_{i^{\prime} i}^{2}(p, K)=\sum_{j=K}^{2 N_{A L^{-1}}} s_{j}(p)\left\{\sqrt{w_{i^{\prime}}} P_{j K}\left(x_{i^{\prime}}\right)\left[\hbar^{2} j(j+1)\right] P_{j K}\left(x_{i}\right) \sqrt{w_{i}}\right\} .
$$

With the phase convention that $P_{j K}(\cos \gamma)=\sqrt{2 \pi} Y_{j K}(\gamma, 0)$, where $Y_{l m}(\theta, \phi)$ is the usual spherical harmonic [45], the symmetry factor $s_{j}(p)$ is given by $s_{j}(p)=1+$ $(-1)^{j+p}$.

We now discuss the grid representation of the Coriolis coupling. For the present discussion we consider only the " + " Coriolis term [i.e. the second line in Eq. (5.18)], leaving the detailed derivation of the "-" Coriolis term as an exercise for the reader. In addition, we focus on the $\hat{W} \equiv\left[\delta_{K^{\prime}, K+1} \times \hat{j}^{+}\right]$portion of the "+" Coriolis term, since the remaining factors are diagonal in the present treatment. Before beginning the derivation, we note that the differential operator corresponding to the body-fixed $\hat{j}^{+}$depends only on the polar angle $\gamma$. This may seem strange since raising and lowering operators usually depend on both the polar and azimuthal angles which define the rotation. However, our "three angle" [i.e. $(\Phi, \Theta, \Psi)]$ definition of the body-fixed frame replaces the $\Psi$ dependence of $\hat{j}^{+}$with $K$ dependence. There is an analogous "two angle" [i.e. $(\Phi, \Theta)$ ] definition of the body-fixed frame in which $\hat{j}^{+}$in that reference frame does depend on both the polar angle $\gamma$ and the azimuthal angle $\Psi[46]$.

The finite basis representation (FBR) of the " + " Coriolis term using a symmetry adapted AL basis set with symmetry $p$ gives

$$
W_{j^{\prime} K^{\prime}, j K}^{F B R}(p)=\delta_{K^{\prime}, K+1} \sum_{i=0}^{2 N_{A L}-1} \sqrt{w_{i}} P_{j^{\prime} K^{\prime}}^{p}\left(x_{i}\right)\left[\hbar \Lambda_{j K}^{+}\right] P_{j, K+1}^{p}\left(x_{i}\right) \sqrt{w_{i}},
$$

where $P_{j K}^{p}(x) \equiv \sqrt{s_{j}(p)} P_{j K}(x)$. After applying the $\delta_{K^{\prime}, K+1}$ to the $K^{\prime}$ dependence of the summand, this expression reduces to

$$
W_{j^{\prime} K^{\prime}, j K}^{F B R}(p)=\delta_{K^{\prime}, K+1} \delta_{j^{\prime}, j} s_{j}(p) \hbar \Lambda_{j K}^{+} .
$$


Now performing the FBR to DVR transformation and applying the $\delta_{K^{\prime}, K+1}$ to the resulting $K^{\prime}$ dependence, we get $W_{i^{\prime} K^{\prime}, i K}^{D V R}(p)=\delta_{K^{\prime}, K+1} \times j_{i^{\prime} i}^{+}(p, K)$ defined by

$$
j_{i^{\prime} i}^{+}(p, K)=\sum_{j=K+1}^{2 N_{A L}-1} s_{j}(p)\left\{\sqrt{w_{i^{\prime}}} P_{j, K+1}\left(x_{i^{\prime}}\right)\left[\hbar \Lambda_{j K}^{+}\right] P_{j K}\left(x_{i}\right) \sqrt{w_{i}}\right\} .
$$

To complete the definition of the basis set, we note that $j_{i^{\prime} i}^{-}(p, K)=j_{i^{\prime}}^{+}(p, K-1)$.

The primitive grid is truncated in the following fashion. For each DVR grid point in the primitive grid, a diagonal element of

$$
\hat{T}^{\gamma}+\frac{\hbar^{2}}{2 \mu R^{2}} J(J+1)+\hat{V}(r, R, \gamma)
$$

is constructed. If that energy exceeds some input $V_{\text {cut }}$, the point is discarded; otherwise it is retained in the basis [47]. Also, if a point is asymptotic, based on some convergence criterion related to the definition of the $A B C$, it is also discarded. In this way, the grid is tailored to the shape of the PES and the ABC. The sparse matrixvector multiply with a truncated DVR grid was first discussed by Groenenboom et al. $[40,41]$, and their method is adopted here.

To complete the definition of the truncated basis set, we consider the allowed values of $K$, the body-fixed projection quantum number. In principle $K=0, \ldots, J$ for even $J+P$ and $1, \ldots, J$ for odd $J+P$. With a finite basis for the Jacobi angle, however, $K$ can not exceed $\min \left(J, 2 N_{A L}-1\right)$. We have found that for the reaction probabilities considered in the present Chapter, convergence is reached with $K_{\text {max }}=2$, in accord with the basis set contraction results of Zhang [35]. This rapid convergence with respect to $K_{\max }$ facilitates exact calculations with very modest increases in CPU time as $J$ increases, and is one of the many useful aspects of the body-fixed representation.

\subsubsection{The Reference Scattering State}

The reference scattering state can be chosen as a distorted wave (with any level of distortion) or as a free wave, as long as it is regular at the origin and is an eigenstate of the asymptotic Hamiltonian, $\hat{H}_{0}=\lim _{R \rightarrow \infty} \hat{H}$. Groenenboom et al. 
[41] and Thompson and Miller [28] have found it very useful to use inelastically distorted waves in their reactive scattering calculations, because they could represent the chemical reaction by focusing on the local exchange region. For the simplicity of the present application we use (almost) free waves, including only the centrifugal phase shift. This is the lowest level of distortion which facilitates practical calculations.

With this level of distortion, the reference scattering state is the product of a translational state, a vibrational state, and a rotational state:

$$
\left|\Phi_{v, j, l}^{J M}\left(E_{t}\right)\right\rangle=\left|h_{l}\left(E_{t}\right)\right\rangle\left|\phi_{v, j}\right\rangle|J M j l\rangle
$$

For the translational function, we choose the spherical Hankel function of the second kind $\left\{h_{l}^{(2)}(x)\right\}[45]$. properly normalized to give unit incoming flux:

$$
\left\langle R \mid h_{l}\left(E_{t}\right)\right\rangle \equiv \frac{-i k R}{\sqrt{v_{t}}} h_{l}^{(2)}(k R) \sim \frac{1}{\sqrt{v_{t}}} e^{-i(k R-l \pi / 2)}
$$

In Eq. (5.26), $k=\sqrt{2 \mu E_{t}} / \hbar$ and $v_{t}=\hbar k_{t} / \mu_{t}$, where $\mu_{t}$ and $k_{t}$ are defined in Eqs. (5.4) and (5.5), respectively. We note that these are the same incoming-wave boundary conditions used in many of the $S$-matrix Kohn variational principle calculations [16]. Also, in Eq. (5.25), $\left|\phi_{v, j}\right\rangle$ is the diatomic rovibrational state.

The rotational state $|J M j l\rangle$ in Eq. (5.25) is a SF coupled eigenstate of $\hat{J}^{2}$, $\hat{J}_{Z_{S F}}, \hat{j}^{2}$, and $\hat{L}^{2}$. This asymptotic rotational state is used in most modern reactive scattering calculations [48]. It is useful for three reasons. First, it exploits the fact that $J$ is conserved. Second, in the ABC formulation of quantum scattering, the absorbing potential may be non-zero only for values of $R$ large enough that the asymptotic state nearly solves the Schrödinger equation. The term in the Hamiltonian which is responsible for mixing $l$-states is the PES, which is typically a much shorter ranged interaction than the $1 / R^{2}$ term which couples the BF labels $\{K\}$. As such, the $\mathrm{SF}$ representation allows the use of smaller $L^{2}$ basis sets. Third, the strength of the coupling which mixes the $\{K\}$ manifold increases as $\Lambda_{J K^{*}}^{ \pm} \sim J$ [cf. Eqs. (5.18) and (5.19)], and as such would require re-optimization of the basis set and absorbing potential for each value of $J$. Thus, the SF representation allows us to use a single, relatively small $L^{2}$ basis for all values of $J$. 
We have argued for the use of an asymptotic state with SF labels. However, we must represent this state with $\mathrm{BF}$ angles since these are used to simplify the Hamiltonian by integrating out overall rotation. This was done in 1968 by Miller [48]. We give a detailed derivation of the position representation of the angular state $|J M j l\rangle$ using $\mathrm{BF}$ angles, i.e. $(\Phi, \Theta, \Psi, \gamma)$. This is useful because it demonstrates the relationship between $\mathrm{BF}$ and SF labels. It also indicates how symmetry is used in the present calculations. The derivation involves three steps. First, we write down the coupled eigenfunction using SF angles. We then express the SF angles as rotated $\mathrm{BF}$ angles. Finally we use Clebsch-Gordan coefficients and orthognnality relations to simplify the result. Recalling that $(\Phi, \Theta)$ are the $\mathrm{SF}$ angles of $\mathbf{R}$ and defining $(\phi, \theta)$ as the SF angles of $\mathbf{r}$, we have the well known result for the coupled angular momentum eigenfunction $\mathcal{Y}_{j l}^{J M}(\Phi, \Theta, \phi, \theta) \equiv\langle\Phi, \Theta, \phi, \theta \mid J M j l\rangle$ :

$$
\mathcal{Y}_{j l}^{J M}(\Phi, \Theta, \phi, \theta)=\sum_{m=-j}^{j} Y_{j m}(\theta, \phi) Y_{l, M-m}(\Theta, \Phi) C(j, l, m, M-m \mid j, l, J, M),
$$

where the Clebsch-Gordan coefficient $C\left(j_{1}, j_{2}, m_{1}, m_{2} \mid j_{1}, j_{2}, j, m\right)$ is a vector coupling coefficient for the addition of angular momenta $\hat{j}_{1}$ and $\hat{\mathbf{j}}_{2}$ to yield the total angular momentum $\hat{\mathbf{j}}$. For a subsequent step in the derivation, it is convenient to express $Y_{l, M-m}(\Theta, \Phi)$ in terms of a Wigner function via

$$
Y_{l, M-m}(\Theta, \Phi)=\sqrt{\frac{2 l+1}{4 \pi}}\left[D_{M-m, 0}^{l}(\Phi, \Theta, 0)\right]^{*}
$$

with the Wigner function defined in Eqs. (5.14) and (5.15). Next, we express $Y_{j m}(\theta, \phi)$ as a rotated spherical harmonic with BF angles via

$$
Y_{j m}(\theta, \phi)=\sum_{K=-j}^{j} Y_{j K}(\gamma, \Psi) D_{K, m}^{j}(0,-\Theta,-\Phi) .
$$

This follows from the fact that the SF and BF angles of $\mathbf{r}$ transform via

$$
|\theta, \phi\rangle=\hat{\mathcal{R}}(\Phi, \Theta, 0)|\gamma, \Psi\rangle
$$

It may seem that we are using a two angle rotation, which is inconsistent with the construction of the BF Hamiltonian. In fact, we eventually use a three angle rotation, applying the third rotation trivially as the final step in the derivation. 
We can now begin to construct the SF rotational function with BF angles. To do this we note that given $(\Theta, \Phi)$, we can take either $(\theta, \phi)$ or $(\gamma, \Psi)$ as the other two independent angles. As such, whenever we use the angles $(\gamma, \Psi)$ as independent variables, we denote $(\tilde{\theta}, \tilde{\phi})$ as the values taken by the transformation function which gives the SF angles in terms of the BF angles, i.e. that defined in Eq. (5.30). We can then define the rotational function $\mathcal{X}_{j l}^{J M}(\Phi, \Theta, \Psi, \gamma) \equiv \mathcal{Y}_{j l}^{J M}(\Phi, \Theta, \tilde{\phi}, \tilde{\theta})$. Using the unitarity of the rotation operator $\hat{\mathcal{R}}$ and Eqs. (5.28) and $(5.29), \mathcal{X}_{j, l}^{J M}(\Phi, \Theta, \Psi, \gamma)$ is given by

$$
\begin{aligned}
\mathcal{X}_{j l}^{J M}(\Phi, \Theta, \Psi, \gamma) & =\sqrt{\frac{2 l+1}{4 \pi}} \sum_{m=-j}^{j} \sum_{K=-j}^{j} Y_{j K}(\gamma, \Psi) C(j, l, m, M-m \mid j, l, J, M) \\
& \times\left\{D_{M-m, 0}^{l}(\Phi, \Theta, 0) D_{m, K}^{j}(\Phi, \Theta, 0)\right\}^{*}
\end{aligned}
$$

This simplifies by using the angular momentum coupling relation for Wigner rotation matrix elements:

$$
\begin{aligned}
& D_{M-m, 0}^{l}(\Phi, \Theta, 0) D_{m, K}^{j}(\Phi, \Theta, 0)= \\
& \sum_{J^{\prime}=|j-l|}^{j+l} C\left(j, l, m, M-m \mid j, l, J^{\prime}, M\right) D_{M, K}^{J^{\prime}}(\Phi, \Theta, 0) C\left(j, l, K, 0 \mid j, l, J^{\prime}, K\right) .
\end{aligned}
$$

Simplification occurs by plugging in this result and using Clebsch-Gordan orthogonality relations:

$$
\begin{aligned}
\mathcal{X}_{j l}^{J M}(\Phi, \Theta, \Psi, \gamma)= & \sqrt{\frac{2 l+1}{4 \pi} \sum_{K=-j}^{j} Y_{j K}(\gamma, \Psi)} \\
& \times \sum_{J^{\prime}=|j-l|}^{j+l}\left[D_{M, K}^{J^{\prime}}(\Phi, \Theta, 0)\right]^{*} C\left(j, l, K, 0 \mid j, l, J^{\prime}, K\right) \\
& \times \sum_{m=-j}^{j} C\left(j, l, m, M-m \mid j, l, J^{\prime}, M\right) C(j, l, m, M-m \mid j, l, J, M) \\
= & \sqrt{\frac{2 l+1}{4 \pi} \sum_{K^{\prime}=-j}^{j} Y_{j K}(\gamma, \Psi)} \\
& \times \sum_{J^{\prime}=|j-l|}^{j+l}\left[D_{M, K}^{J^{\prime}}(\Phi, \Theta, 0)\right]^{*} C\left(j, l, K, 0 \mid j, l, J^{\prime}, K\right) \times \delta_{J^{\prime}, J}
\end{aligned}
$$




$$
\begin{aligned}
= & \sqrt{\frac{2 l+1}{4 \pi}} \\
& \times \sum_{K=-j}^{j} Y_{j K}(\gamma, \Psi)\left[D_{M, K}^{J}(\Phi, \Theta, 0)\right]^{*} C(j, l, K, 0 \mid j, l, J, K) .
\end{aligned}
$$

This is almost the final form for the rotational function. To complete the derivation, we note that

$$
\frac{1}{\sqrt{4 \pi}} Y_{j K}(\gamma, \Psi)\left[D_{M, K}^{J}(\Phi, \Theta, 0)\right]^{*}=\frac{1}{\sqrt{2 J+1}} P_{j K}(\cos \gamma) \tilde{D}_{M, K}^{J}(\Phi, \Theta, \Psi)
$$

where $P_{j K}(\cos \gamma)$ and $\tilde{D}_{M, K}^{J}(\Phi, \Theta, \Psi)$ are defined in Eqs. (5.21) and (5.16) respectively. The final form for the rotational function is then

$$
\begin{aligned}
\mathcal{X}_{j l}^{J M}(\Phi, \Theta, \Psi, \gamma)= & \sqrt{\frac{2 l+1}{2 J+1}} \sum_{K=-j}^{j} P_{j K}(\cos \gamma) \tilde{D}_{M, K}^{J}(\Phi, \Theta, \Psi) \\
& \times C(j, l, K, 0 \mid j, l, J, K) .
\end{aligned}
$$

This final step completes the three angle rotation used to define the SF function with $\mathrm{BF}$ angles.

We make four general comments about the rotational function in Eq. (5.35), for which the demonstration is left as an exercise for the reader. First, this rotational function is an eigenfunction of the parity operator with parity $P=j+l$. This is plausible because the sum ranges over positive and negative values of $K$, as does the sum which defines the parity adapted Wigner function defined in Eq. (5.17). The ClebscllGordan coefficients have the required symmetry properties [36] to give definite parity. Second, this rotational function also has definite symmetry when exchanging atoms $B$ and $C$ [see Fig. J.1]. Such an exchange maps $\cos \gamma \mapsto-\cos \gamma$, giving an overall phase factor $(-1)^{j}$ from our definition of $P_{j K}(\cos \gamma)$. Thus, the rotational function has exchange symmetry $p=j$. Third, $\left[P_{j K}(\cos \gamma) \times \tilde{D}_{M, K}^{J}(\Phi, \Theta, \Psi)\right]$ is a rotational function with BF labels and angles, whereas the function in Eq. (5.35) has SF labels and BF angles. Therefore the Clebsch-Gordan coefficients furnish the transformation from SF labels to BF labels. Indeed, one can say that the coefficients $\{C(j, l, K, 0 \mid j, l, J, K)\}$ transform between the " $K$ " and " $l$ " representations. Finally, based on the constraints of vector addition which determine the non-zero Clebsch-Gordan coefficients, the sum over $K$ has non-zero contribution from only $K=0, \pm 1, \pm 2, \ldots, \pm \min (j, J)$. 
To complete the discussion of the frame transformation and the use of symmetry in the present calculations, we indicate the transformation between a BF rotational state and the SF rotational state. We label the BF rotational state by $|J K P i p\rangle$ where $K P$ labels a symmetrized Wigner state with inversion symmetry $P$, and ip labels a symmetrized AL DVR state with exchange symmetry $p$. Computing the transformation element is made easy by inserting unity resolved in BF angles $\Omega$ $\equiv(\Phi, \Theta, \Psi, \gamma)$

$$
\begin{aligned}
\langle J M K P i p \mid J M j l\rangle & =\int d \Omega\langle J M K P i p \mid \Omega\rangle\langle\Omega \mid J M j l\rangle \\
& =\int d \Omega \mathcal{Q}_{i, K}^{J M P p}(\Phi, \Theta, \Psi, \gamma) \mathcal{X}_{j, l}^{J M}(\Phi, \Theta, \Psi, \gamma) .
\end{aligned}
$$

In Eq. (5.36), $\mathcal{X}_{j, l}^{J M}(\Phi, \Theta, \Psi, \gamma)$ is given by Eq. (5.35), and $\mathcal{Q}_{i, K}^{J M P p}(\Phi, \Theta, \Psi, \gamma)$ is the $\mathrm{BF}$ function given by

$$
\mathcal{Q}_{i, K}^{J M P p}(\Phi, \Theta, \Psi, \gamma) \equiv A_{i, K}^{p}(\cos \gamma) B_{M, K}^{J P}(\Phi, \Theta, \Psi)
$$

where $B_{M, K}^{J P}(\Phi, \Theta, \Psi)$ is the parity adapted Wigner function defined in Eq. (5.17). Finally, $A_{i, K}^{p}(\cos \gamma)$ is the AL DVR basis function with exchange symmetry $p$, and corresponding to grid point $i$. By analytically integrating over the Euler arigles and using the angular grid tc integrate over $\cos \gamma$, the transformation becomes:

$$
\begin{array}{r}
\langle J M K P i p \mid J M j l\rangle=\left\{\sqrt{w_{i}} P_{j}^{K}\left(x_{i}\right)\right\} \times\left\{\sqrt{\left.\frac{2 l+1}{2 J+1} C(j, l, K, 0 \mid j, l, J, K)\right\} \times}\right. \\
\left\{\frac{1}{\sqrt{2}}\left[1+(-1)^{j+p}\right]\right\} \times\left\{\frac{1}{\sqrt{2\left(1+\delta_{K, 0}\right)}}\left[1+(-1)^{j+l+P}\right]\right\} .
\end{array}
$$

The transformation element is completely independent of $M$, as is the Hamiltonian operator in Eq. (5.18). This demonstrates that the reaction probabilities are independent of $M$. Formally they must be, since our choice of SF z-axis orientation should not affect the dynamics. The first factor in Eq. (5.38) is the usual DVR-FBR transformation element [39]. The second factor is the frame transformation. The remaining factors demonstrate the relationship between the symmetry of the BF rotational state and the SF rotational state. In particular, since the SF rotational state has both definite exchange and inversion symmetry, it projects onto the block of the Hamiltonian 
with exchange symmetry $p=j$ and inversion symmetry $P=j+l$. Using the proper symmetry blocks, these factors are $\sqrt{2}$ for $K=0$ and 2 for $K>0$, multiplying the reaction probability in Eq. (5.7) by 2 and 4, respectively. Thus, the $\mathrm{D}+\mathrm{H}_{2}$ initial state selection allows the calculation of properly symmetrized reaction probabilities while only explicitly treating ca. $\frac{1}{4}$ of the full Hamiltonian.

We summarize our use of different rotational reference frames in reactive scattering. Our purpose is to choose basis functions for overall rotation which minimize coupling in the Hamiltonian. To make the discussion concrete, we define the orbital angular momentum vector operator $\hat{\mathbf{L}} \equiv \hat{\mathbf{J}}-\hat{\mathbf{j}}$. The choice of reference frame is determined by the competition between the PES $\hat{V}$ and the orbital kinetic energy $\hat{L}^{2} / 2 \mu R^{2}$. When choosing basis functions to represent the motion of the system when the collision partners are strongly interacting, the $\mathrm{BF}$ frame is preferable. In this case, $\hat{V} \gg \hat{L}^{2} / 2 \mu R^{2}$. The BF frame is diagonal in $\hat{V}$, and thus diagonalizes most of the Hamiltonian. Alternatively, when choosing basis functions to represent the motion of the system when the collision partners are well separated, the SF frame is preferable. In this case, $\hat{L}^{2} / 2 \mu R^{2} \gg \hat{V}$. The SF frame is diagonal in $\hat{L}^{2} / 2 \mu R^{2}$, and thus diagonalizes most of the Hamiltonian in this case. Finally, one may regard the SF asymptotic rotational function as an analytically distorted wave, with the ClebschGordan transformation from the $K$ representation to the $l$ representation providing the distortion.

\subsubsection{The Absorbing Potential}

The optimum absorbing potential is one which absorbs all outgoing flux with negligible back reflection, as fast (in space) as possible. Several studies have sought reliable guidelines for determining optimal absorbing potentials $[32,49-52]$. We have found excellent convergence behavior with a quartic function:

$$
\epsilon[z(\mathbf{q})]=\lambda\left[\frac{z-z_{0}}{z_{\max }-z_{0}}\right]^{4}
$$

where $z=\max \left[R_{a}, R_{b}(\mathbf{q}), R_{c}(\mathbf{q})\right]$, and $(a, b, c)$ label the three chemical arrangements. The parameters $\left(\lambda, z_{0}, z_{\text {max }}\right)$ are different in different arrangements. They are set to 
give more gentle absorption in the reactant arrangement $(R e=a)$ than in the product arrangements $(\operatorname{Pr}=b, c)$, as demanded by the initial state selection. The parameter $z_{m a x}$ determines the end of the grid in a particular arrangement. It is smaller in the product arrangements where no state selection is required. Converged values of these parameters will be reported below.

\subsubsection{Summary of the Methodology}

With the vector $i \epsilon \Phi_{v, j, l}^{J}\left(E_{t}\right)$ now defined, we use the Newton method to apply $\mathrm{G}(E)$, thus giving the $\mathrm{ABC}$ scattering wavefunction and the reaction probability. The partial wave expansion and the Boltzmann average over relative translational energy and initial rotation give the desired rate constant.

\subsection{Results and Discussion}

We now present the results of our quantum reactive scattering calculations on the $\mathrm{D}+\mathrm{H}_{2}(v=1, j)$ system. As stated in the Introduction, the present Chapter has two main goals. The first is to demonstriste the efficiency of the present method in a non-trivial application. For this purpose, we report the $\mathrm{D}+\mathrm{H}_{2}$ reaction probabilities $P_{v, j, l}^{J}\left(E_{t}\right)$ and cross sections $\sigma_{v, j}\left(E_{t}\right)$, in addition to the typical amounts of core memory and CPU time required for these calculations. The second objective is to determine the $j$ and $T$ dependence of $k_{v=1, j}(T)$, for the purpose of comparison with both experiment and approximate theory.

\subsubsection{Reaction Probabilities and Cross Sections}

We have obtained converged reaction probabilities and cross sections according to Eqs. (5.7) and (5.5), respectively. There are 13 convergence parameters to optimize. These fall into four roughly independent groups: ABC parameters $\left(\lambda^{R e}, \lambda^{P r}, z_{0}^{R e}, z_{0}^{P r}\right)$ for defining the absorbing potential [cf. Eq. (5.39)], parameters $\left(N_{B}, N_{A L}\right)$ for constructing the primitive basis; parameters $\left(z_{\max }^{R e}, z_{\max }^{P r}, V_{c u t}, K_{\max }\right)$ for truncating the basis; and, the Newton inversion parameters $\left(f_{\text {newt }}, \tau_{s}, \delta\right)$ defined 


\begin{tabular}{||c|c|c||}
\hline \hline Absorbing potentials & $\lambda^{R e}=1.8 \times E$ & $\lambda^{P r}=1.0 \times E$ \\
\hline$\underline{\text { Primitive basis set }}$ & $z_{0}^{R e}=4.5 a_{0}$ & $z_{0}^{P r}=3.9 a_{0}$ \\
\hline Truncated basis set & $N_{B}=3.7$ & $N_{A L}=7$ \\
\hline Newton inversion & $z_{\text {max }}^{R e}=(7.4-10.4) a_{0}$ & $z_{\max }^{P r}=(5.5-8.5) a_{0}$ \\
\hline Nut & $\tau_{s}=(2.5-4.2) \mathrm{eV}$ & $K_{\max }=2$ \\
\hline & $\delta=2 \times 10^{-2}$ & $f_{\text {newt }}=1.6$ \\
\hline
\end{tabular}

Table 3.1: Optimized convergence parameters for the present quantum reactive scattering calculations. These values are sufficient to give better than $3 \%$ accuracy for $0.15 \mathrm{eV}<E_{t}<0.37 \mathrm{eV}$, and better than $6 \%$ accuracy otherwise. $E=$ total scattering energy, $R e$ is reactant, and $\operatorname{Pr}$ is product.

in Ref. 53 and in the previous Chapter. Table 5.1 shows the optimized values. These parameters are sufficient to obtain better than $3 \%$ accuracy for the lower translational energies $\left(0.15 \mathrm{eV}<E_{t}<0.37 \mathrm{eV}\right)$, and better than $6 \%$ accuracy for the higher translational energies. We focus attention on the truncation parameters.

The parameters $\left(z_{\max }^{R e}, z_{\max }^{P r}, V_{c u t}\right)$ require careful optimization. For small $E_{t}$, the initial translational energy, $z_{\max }^{R e}$ and $z_{\max }^{P_{r}}$ must be large enough to encompass the long de Broglie wavelengths. Alternatively, for larger $E_{t}$, the parameter $V_{\text {cut }}$ must be set to allow the wavefunction to sample larger portions of the PES. This competition between small and large translational energies caused the truncated grid sizes to be roughly independent of the energy, with grid sizes falling in the range $N_{\text {grid }}=6500 \pm 1500$. 


\section{Reaction Probabilities}

We demonstrate the convergence of selected reaction probabilities with respect to $\left(z_{\max }^{R e}, z_{\max }^{P r}\right)$, which control the spatial extent of the grid and the strength of the absorbing potential. As $\left(z_{\text {max }}^{R e}, z_{\text {max }}^{P r}\right)$ increase the grid samples more space, the absorbing potential becomes more gentle, and the reaction probabilities should converge to the exact values. Since we ultimately compute thermal rate constants, we need to converge reaction probabilities with small initial translational energies. For example, the $\mathrm{D}+\mathrm{H}_{2}(v=1, j=0)$ channel becomes energetically accessible for total energy $E>0.786 \mathrm{eV}$ [where $V\left(r=r_{e q}, R=\infty, \gamma\right)=0$ defines the zero of total energy]. The threshold for this reaction probability occurs in the total energy range $E \in(0.85 \mathrm{eV}, 0.90 \mathrm{eV})$. Fig. 5.2 demonstrates the convergence behavior of the reaction probabilities $P_{v=1, j=0, l=0}^{J=0}(E)$ for $E=0.85$ and $0.88 \mathrm{eV}$. These calculations used $V_{c u t}=2.8 \mathrm{eV}$. Fig. 5.2 shows that large grids are required to represent the long de Broglie wavelengths for $E=0.85 \mathrm{eV}$. Even a small change in energy from $E=0.85 \mathrm{eV}$ to $0.88 \mathrm{eV}$ allows the use of smaller grids. We also see that the $E=0.85 \mathrm{eV}$ probability is more stable when turning on the absorbing potential slightly farther into the asymptotic region. Finally, Fig. 5.2 shows that the $E=0.85 \mathrm{eV}$ probability is known roughly to within a factor of 2 , whereas the $E=0.88 \mathrm{eV}$ probability is known to within $10 \%$. Fortunately, we are not interested in temperatures low enough that these probabilities give the dominant contribution. In fact, these are the smallest reaction probabilities used to compute the rate constants reported below.

Reaction probabilities which give dominant contributions to their respective rate constants at $T=300 \mathrm{~K}$ are $P_{v=1, j=0, l=0}^{J=0}(E=1.0 \mathrm{eV}), P_{v=1, j=1, l=5}^{J=6}(E=1.014 \mathrm{eV})$, and $P_{v=1, j=1, l=8}^{J=9}(E=1.104 \mathrm{eV})$. The convergence of these reaction probabilities with respect to $\left(z_{\max }^{R e}, z_{\max }^{P r}\right)$ is shown in Fig. 5.3, using $V_{\text {cut }}=2.8 \mathrm{eV}$ and $\left(z_{0}^{R e}, z_{0}^{P r}\right)=$ $\left(4.5 a_{0}, 3.9 a_{0}\right)$. These reaction probabilities can be obtained with significantly smaller grids than are sequired for the threshold probabilities shown in Fig. 5.2. In addition, these probabilities are stable to roughly $3 \%$ with respect to chinges in $\left(z_{\max }^{R e}, z_{\max }^{P r}\right)$. Since these are the most important probabilities for the subsequent rate constant calculations, we expect the rate constants to be accurate to roughly $3 \%$ as well. 


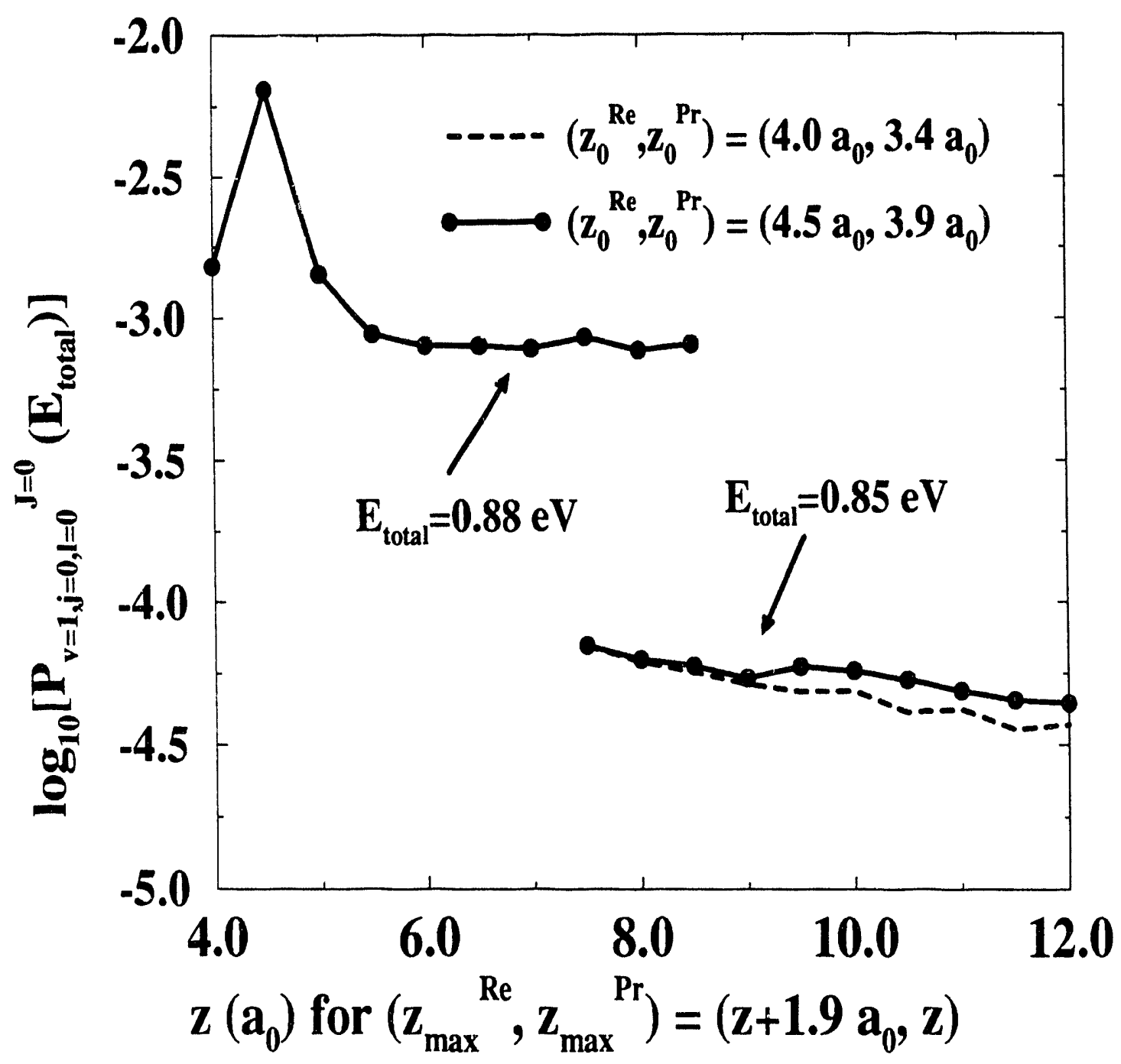

Figure 5.2: Convergence with respect to $\left(z_{\max }^{R e}, z_{\max }^{P r}\right)$ of the $\mathrm{D}+\mathrm{H}_{2}(v=1, j=0, l=0)$ reaction probabilities at total energy $E=0.85$ and $0.88 \mathrm{eV}$. Large grids are required to converge very small reaction probabilities. The convergence is more stable when the absorbing potentials turn on farther out in the asymptotic region of space. The $E=0.85 \mathrm{eV}$ probability is known roughly to within a factor of 2 , whereas the $E=$ $0.88 \mathrm{eV}$ probability is known to within $10 \%$. 


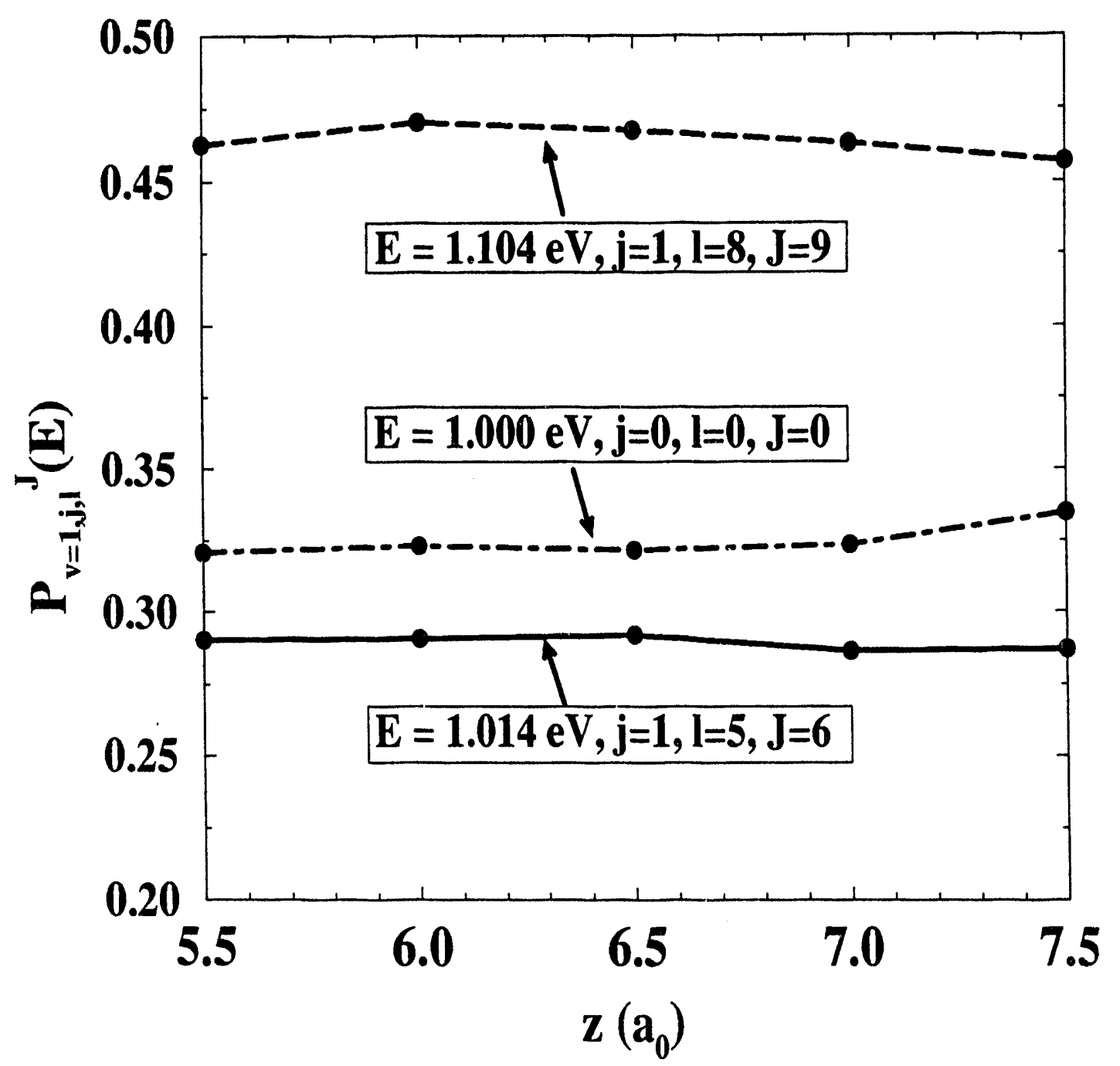

Figure 5.3: Convergence with respect to $\left(z_{\max }^{R e}, z_{\max }^{P r}\right)$ of the $\mathrm{D}+\mathrm{H}_{2}$ reaction probabilities $P_{v=1, j=0, l=0}^{J=0}(E=1.0 \mathrm{eV}), P_{v=1, j=1, l=5}^{J=6}(E=1.014 \mathrm{eV})$, and $P_{v=1, j=1, l=8}^{J=9}(E=$ $1.104 \mathrm{eV})$. The abscissa $z$ has the same meaning as in Fig. 5.2, i.e. $\left(z_{\max }^{R e}, z_{\max }^{P_{r}}\right)=$ $\left(z+1.9 a_{0}, z\right)$. These probabilities give significant contribution to the rate constants reported below. Small grids can be used to obtain these probabilities, which are at energies above threshold. These probabilities are known to roughly $3 \%$. 
Converged reaction probabilties for the $\mathrm{D}+\mathrm{H}_{2}(v=1, j)$ system with total $J=0$ are shown in Fig. 5.4. The $j=0$ probabilities are compared with the $S$-matrix Kohn variational principle (SMKVP) results of Zhang and Miller [16]. The agreement is generally very good over the entire energy range, and is truly excellent for total energies $E<1 \mathrm{eV}$. The $j=1$ probability increases from threshold most rapidly. This phenomenon was observed in the previous Chapter, and was attibruted to a small amount of orbital angular momentum $(l=1)$ present in the initial state, helping to focus the system into more reactive geometries. We note that the $j=2$ energy dependence roughly follows that for $j=0$, and that the $j=3$ curve rises to the same level as that for $j=1$. This may be the result of some approximate symmetry dependent selection rule which determines reactivity. Determining what portion of the Hamiltonian controls this effect may allow estimates of reactivity for more complex systems.

\section{Cross Sections}

In order to obtain the reaction cross section, reaction probabilities with $J \geq 0$ must be obtained to perform the sum over partial cross sections in Eq. (5.5). Zhang has studied the convergence of partial cross sections with repsect to $K_{\max }$ [35]. He found that the $J=10$ partial cross section for $\mathrm{H}+\mathrm{H}_{2}$ at total energy $E=0.6$ and $1.0 \mathrm{eV}$ converges with $K_{\max }=3$ and 4 , respectively. In principle, the optimal value of $K_{\max }$ depends on $E$ and $J$, in addition to the initial rotational quantum number $j$. To avoid such complication, we examined the convergence of $\mathrm{D}+\mathrm{H}_{2}$ full cross sections with $K_{\max }$ for various values of $E$ and $j$. Figure 5.5 shows the convergence of $\sigma_{v=0, j}$ at $E=0.85 \mathrm{eV}$ for $j=(0,1,2)$. We see rapid convergence of these cross sections as $K_{\max }$ increases, gaining nearly three digit accuracy with $K_{\max }=2$. Similar results were obtained at $E=1.1 \mathrm{eV}$, which require $J$ as large as 24 . Based on these results, we have used $K_{\max }=2$ for all subsequent calculations reported in this Chapter.

The partial cross sections required to construct $\sigma_{v=1, j=0}(E)$ are shown in Fig. 5.6 for various total energies. These were computed using $K_{\max }=2$, based on the convergence behavior seen in Fig. 5.5. The linear increase for small $J$ results 


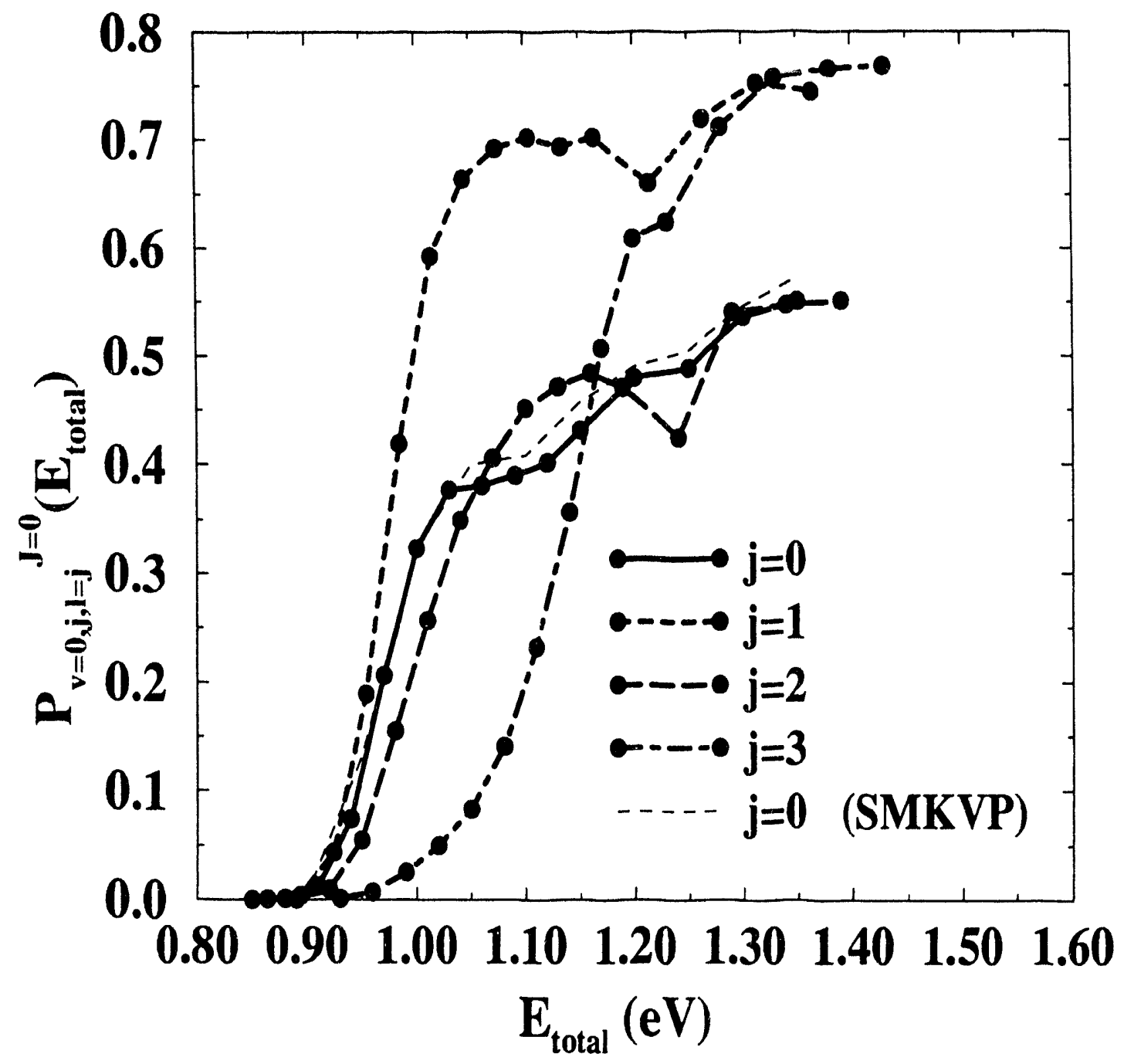

Figure 5.4: Reaction probabilities for the $\mathrm{D}+\mathrm{H}_{2}(v=1, j)$ system. Comparison is made with the $S$-matrix Kohn variational principle (SMKVP) calculations of Zhang and Miller [Ref. 16] for the $j=0$ transition. Excellent agreement is seen, especially for total energies below $1 \mathrm{eV}$. The $j=1$ probability increases from threshold most rapidly, and is matched by the $j=3$ curve at high energy. We attribute this rapid increase in the $j=1$ energy dependence to the result of orbital motion. 


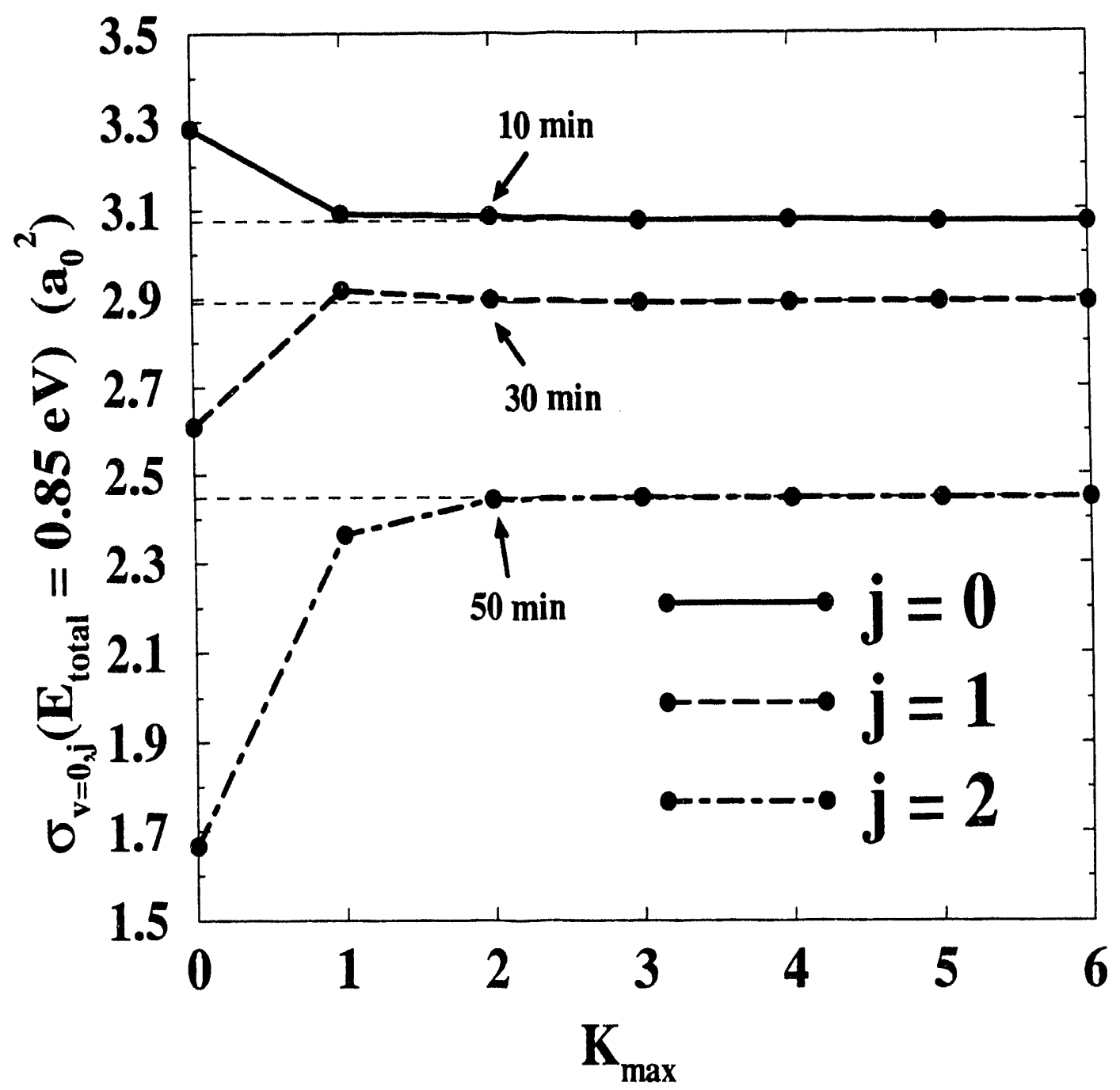

Figure 5.5: Convergence with respect to $K_{\max }$ of the $\mathrm{D}+\mathrm{H}_{2}(v=0, j)$ reaction cross sections at total energy $E=0.85 \mathrm{eV}$, for $j=(0,1,2)$. We see rapid convergence as $K_{\max }$ increases, gaining nearly three digit accuracy for $K_{\max }=2$. The $j=0$ calculation for $K_{\max }=2$ required only 10 minutes. 
from the product of reaction probabilties weakly dependent on $J$ and the $2 J+1$ SF projection degeneracy. The exponential decrease for larger $J$ values results from the repulsive centrifugal force keeping the reactants well separated. The competition between these two effects gives a different maximum partial cross section for each energy. This maximum occurs at larger $J$ values as energy increases, which is explicitly demonstrated by the two partial cross section curves marked at $E=0.91 \mathrm{eV}$ and $1.35 \mathrm{eV}$. Qualitatively, Fig. 5.6 suggests that for a given value of $J$, reaction is possible only after an energy roughly equal to $J(J+1)$ is spent in overcoming the centrifugal barrier. This idea is made more quantitative below in our discussion of the $J$-shifting approximation.

The $\mathrm{D}+\mathrm{H}_{2}(v=1, j)$ reaction cross sections are shown in Fig. 5.7, as a function of total energy. The thick lines show the present calculations for $j=(0,1,2,3)$, and the thin dotted line is the $j=0$ result of Zhang and Miller obtained from the SMKVP [16]. We see complete agreement for $j=0$ between the two methods over the entire energy range. The discrepancies between the SMKVP and the ABC-DVRNewton method for $J=0$ and $E>1 \mathrm{eV}$ seem to have averaged out in the sum over orbital and total angular momentum.

The initial state selected reaction cross sections in Fig. 5.7 demonstrate the very smooth energy dependence that results from averaging over partial waves and final states. We also see that the cross sections systematically decrease with increasing $j$. This contrasts the $j$ depencince of the $J=0$ reaction probabilities shown in Fig. 5.4, in which probabilities for $j=1$ and 3 are the largest for many energies. The $j$ dependence of the cross sections in Fig. 5.7 results from computing the "helicity averaged" cross section. In principle, the cross section depends on the initial orientation of the diatomic molecular rotation (i.e. BF projection quantum number $K$, or helicity in the language of particle physics). Since most experiments do not prepare reactants with orientational selection, we average over the helicities. This average over $K$ is performed in the $\mathrm{SF}$ representation as discussed above, by averaging over $l$. Different $l$ states have different parities, which can have a large effect on the magnitude of the reaction probabilities. The $j>0$ cross sections involve an average over even and odd parity, whereas the $j=0$ cross sections arise from only 


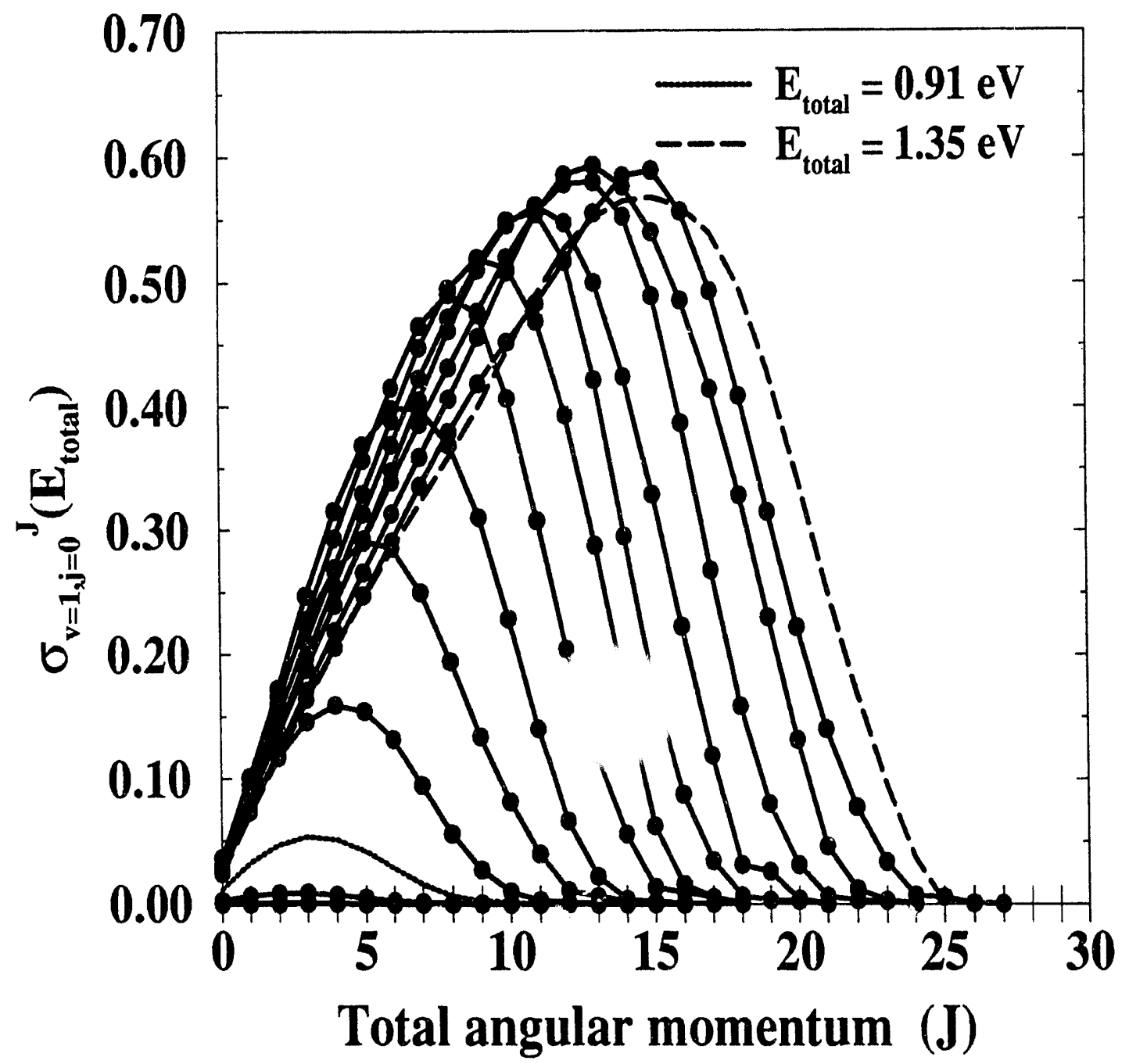

Figure 5.6: The dependence of the partial cross sections $\sigma_{v=1, j=0}^{J}\left(E_{\text {total }}\right)$ on total angular momentum $J$, for various total energies. The linear increase for small $J$ results from the $2 J+1 \mathrm{SF}$ projection degeneracy. The exponential decrease for larger $J$ results from the centrifugal force keeping the reactants well separated. The exponential decrease cccurs at larger $J$ values for larger initial translational energies, as demonstrated by the two marked total energies $E_{\text {total }}=0.91 \mathrm{eV}$ and $1.35 \mathrm{eV}$. 
even parity calculations. Since the odd parity block lacks the $K=0$ component, the transition state is not energetically accessible and the reaction probabilities are quite small.

We now report the computational effort required by these calculations, which were performed on an IBM RS/6000 Model 550. Total propagation times ranged from 60 fs (higher $E_{t}$ ) to 100 fs (lower $E_{t}$ ). This corresponds to the time required for reaction and absorption. The linear system size (i.e. the dimension of the Hamiltonian matrix) ranged from $5000(J=0$, small grid) to $25000(J \geq 2$, large grid). The number of Newton expansions performed for each Green's function calculation varied from 10 to 20 . With $K_{\text {newt }}=f_{\text {newt }} \times \tau_{s}=80$, this corresponds to 800-1600 matrixvector multiplies for each reaction probability.

All timings are for the $j=0$ cross sections. Timings for higher $j$ values are roughly obtained by multplying the $j=0$ timings by $2 j+1$, the number of terms in the average over orbital angular momentum. The cross sections in Fig. 5.5 required ca. $3 \frac{1}{3}$ minutes times $K_{\max }+1$, for a calculation using $K_{\max }$. Thus, converged reaction cross sections are obtained in 10 minutes. The cross sections in Fig. 5.7 are more demanding, however, because the total energy is higher, and the initial translational energy is lower than that in Fig. 5.5. The lower energy cross sections and the very high energy cross sections in Fig. 5.7 required ca. 60 minutes per energy, and those at the intermediate energies required ca. 40 minutes per energy. Furthermore, with respect to core memory, all calculations presented required less than 4.5 Mbyte. This is possible because the Newton method is an iterative algorithm which is based on storing only a small number of vectors. Thus, these very modest memory and time requirements of the $A B C-D V R-N e w t o n$ method suggest that it may be the most direct route to date for calculating accurate reaction cross sections.

\subsubsection{Rate Constants}

We now present the results of our rate constant calculations using Eqs. (5.15.4). In this section we wish to emphasize two comparisons: the present theory vs. experiment, and the present theory vs. an approximate theory. 


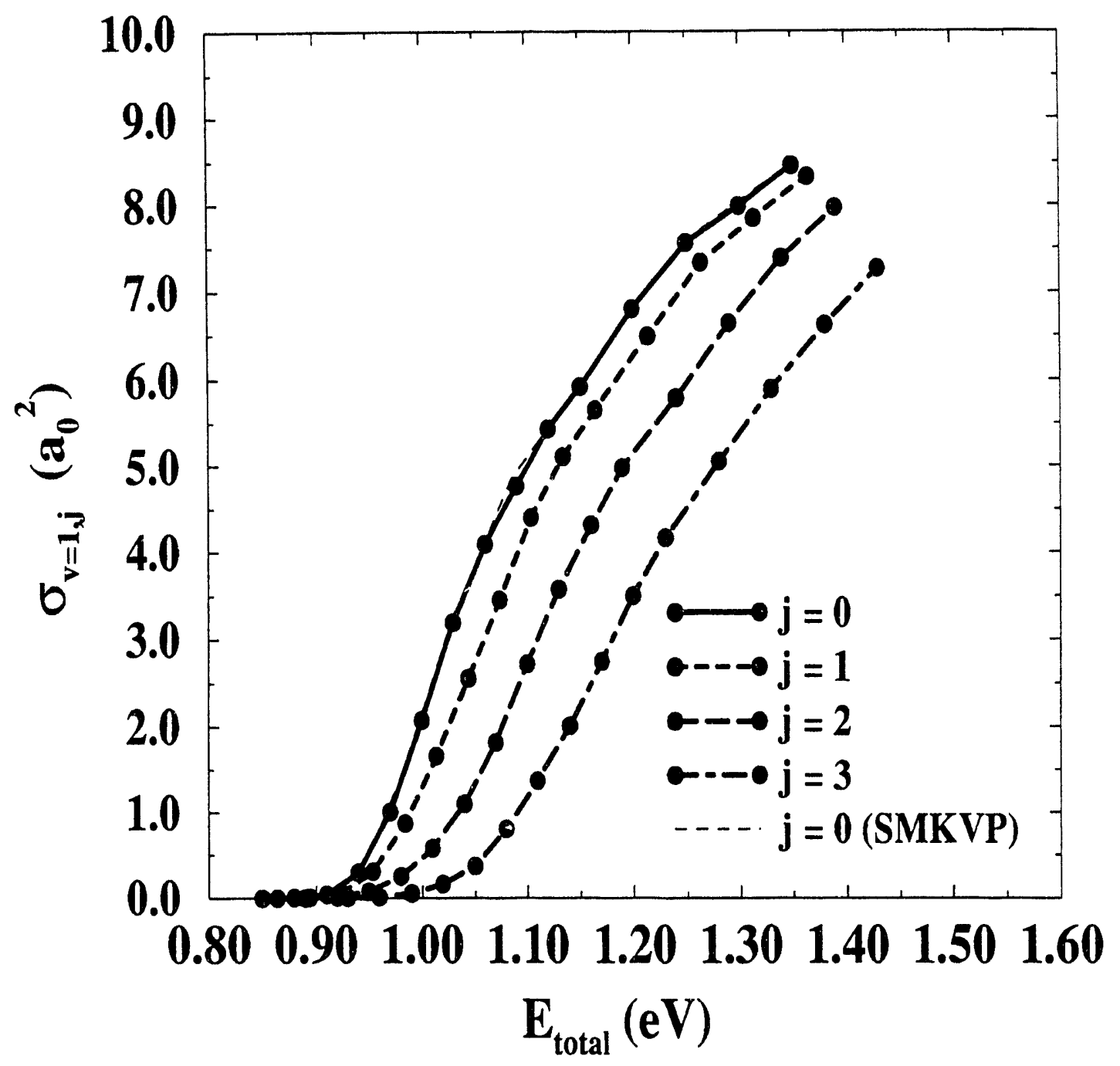

Figure 5.7: Reaction cross sections for $\mathrm{D}+\mathrm{H}_{2}(v=1, j)$ as a function of total energy $(\mathrm{eV})$. The thick lines show the present calculations for $j=(0,1,2,3)$, and the thin dotted line is the $j=0$ SMKVP result of Zhang and Miller [Ref. 16] which agrees completely with the present calculations over the entire energy range. In contrast with the $J=0$ reaction probabilities, the cross sections decrease systematically with increasing $j$ because of symmetry. 
The theory we test is the $J$-shifting approximation (JSA), made popular in quantum reactive scattering theory by Bowman [18, 54] and Schatz [55]. The JSA assumes that $K$ is conserved, and further that the centrifugal coupling is only important near the transition state geometry. Reaction probabilities for $J, K>0$ are obtained by a $(J, K)$-dependent energy shift from the $J=0$ result via [18]

$$
P_{v, j, l}^{J, K}\left(E_{t}\right) \cong P_{v, j, j}^{J=0}\left(E_{t}-\varepsilon_{J, K}\right)
$$

This allows one to estimate observables such as cross sections and rate constants when only accurate $J=0$ calculations are possible. As stated in the Introduction, Bowman [18] and Takada et al. [22] have examined the accuracy of the JSA, in both cases finding good agreement with exact results for low enough energy. The former study tested $\mathrm{H}+\mathrm{H}_{2}$ cumulative reaction probabilities for $J=4$, while the latter examined: $\mathrm{D}+\mathrm{H}_{2}(v=j=0)$ cross sections and rate constants. Our calculation of exact $\mathrm{D}+\mathrm{H}_{2}[v=1, j=(0,1,2,3)]$ rate constants provides an interesting opportunity to test this approximation further.

In this Chapter, we use the linear transition state JSA. This assumes contribution from $K=0$ only, and gives the following $(v, j)$-selected rate constant:

$$
k_{v, j}^{J S A}(T)=A(T) Q_{r o t}^{\ddagger}(T) \int_{0}^{\infty} d E_{t} e^{-E_{t} / k_{B} T} P_{v, j, j}^{J=0}\left(E_{t}\right),
$$

where

$$
A(T)=\sqrt{\frac{8 k_{B} T}{\pi \mu_{t}}}\left(k_{B} T\right)^{-2}\left(\frac{j+1}{2 j+1}\right) \frac{\hbar^{2} \pi}{2 \mu_{t}}
$$

and

$$
Q_{\text {rot }}^{\ddagger}(T)=\sum_{J=0}^{\infty}(2 J+1) e^{-B^{\ddagger} J(J+1) / k_{B} T} .
$$

In Eq. (5.42), the factor of $j+1$ counts the number of $l$-states in the sum over orbital angular momentum which contain a $K=0$ component. In Eq. (5.43), $B^{\ddagger}$ is the rotation constant of the linear transition state species, which is $8.6 \mathrm{~cm}^{-1}$ for the LSTH PES description of $\mathrm{D}+\mathrm{H}_{2}$. The above assumptions are expected to be satisfied at lower temperatures, but less so at higher $t \in m$ peratures. 
Figure 5.8 demonstrates the temperature dependence of exact and JSA rate constants for the $\mathrm{D}+\mathrm{H}_{2}(v=1, j)$ reaction. The temperature range is $200-1000 \mathrm{~K}$, plotted in inverse Kelvin. The exact rate constants were obtained from Eq. (5.4) and the cross sections in Fig. 5.7, and the JSA rate constants were obtained from Eqs. (5.41)-(5.43) and the $J=0$ reaction probabilities in Fig. 5.4. Very good agreement is obtained for all $j$ values. In all cases, the JSA predicts the correct Arrhenius activation energy (i.e. negative of the slope of $\log k(T)$ vs. $1 / k_{B} T$ ), and is qualitatively correct in predicting the Arrhenius prefactor (i.e. $y$-intercept). The overall agreement is truly excellent for $j=1$ and 2 . However, the JSA systematically underestimates the rate constant by roughly $40 \%$ for $j=0$ and 3 .

The activation energies obtained from Fig. 5.8 are all ca. $0.17 \mathrm{eV}$. To put this number in perspective, the activation energy from the $\mathrm{D}+\mathrm{H}_{2}(v=0, j=0)$ Arrhenius plot is $0.32 \mathrm{eV}$ (taken from Table XI in Ref. 16). Due to tunneling, this is slightly less than the classical barrier height on the LSTH PES which is $0.425 \mathrm{eV}$. On the other hand, the $\mathrm{H}_{2}(v=0, j=0) \rightarrow \mathrm{H}_{2}(v=1, j=0)$ vibrational excitation energy is $0.52 \mathrm{eV}$. As such, only $0.15 \mathrm{eV}$ out of $0.52 \mathrm{eV}(29 \%)$ is converted into reactive translational energy. Although the vibrational excitation does enhance reactivity (by three orders of magnitude), it $d$ es so relatively inefficiently. Thus, the $\mathrm{D}+\mathrm{H}_{2}$ system demonstrates a large amount of vibrational adiabaticity.

We now consider experimental results for the $\mathrm{D}+\mathrm{H}_{2}(v=1)$ rate constant. As discussed in the Introduction, the measured rate constant for this reaction has been quite sinsitive to the particular experimental procedure employed [1-3]. Dreier and Wolfrum [4] have measured the rate constant by applying CARS spectroscopy to monitor directly most of the reagents in the system. Since the other experiments have involved indirect probing of some sort [1-3], we consider the CARS measurement to be the most reliable. Thus, we take their result, $k_{v=1}(T=310 K)=(1.9 \pm 0.2) \times 10^{-13}$ $\mathrm{cm}^{3}$ molecule ${ }^{-1} \mathrm{sec}^{-1}$, for comparison.

The most accurate theoretical treatment of this system is by Zhang and Miller [16], who calculated the exact rate constant for $\mathrm{D}+\mathrm{H}_{2}(v=1, j=0)$. Their published result is $k_{v=1, j=0}(T=300 K)=1.63 \times 10^{-13} \mathrm{~cm}^{3}$ molecule ${ }^{-1} \mathrm{sec}^{-1}$. Although the $j$ dependence of this rate constant is not expected to be too strong, a full 


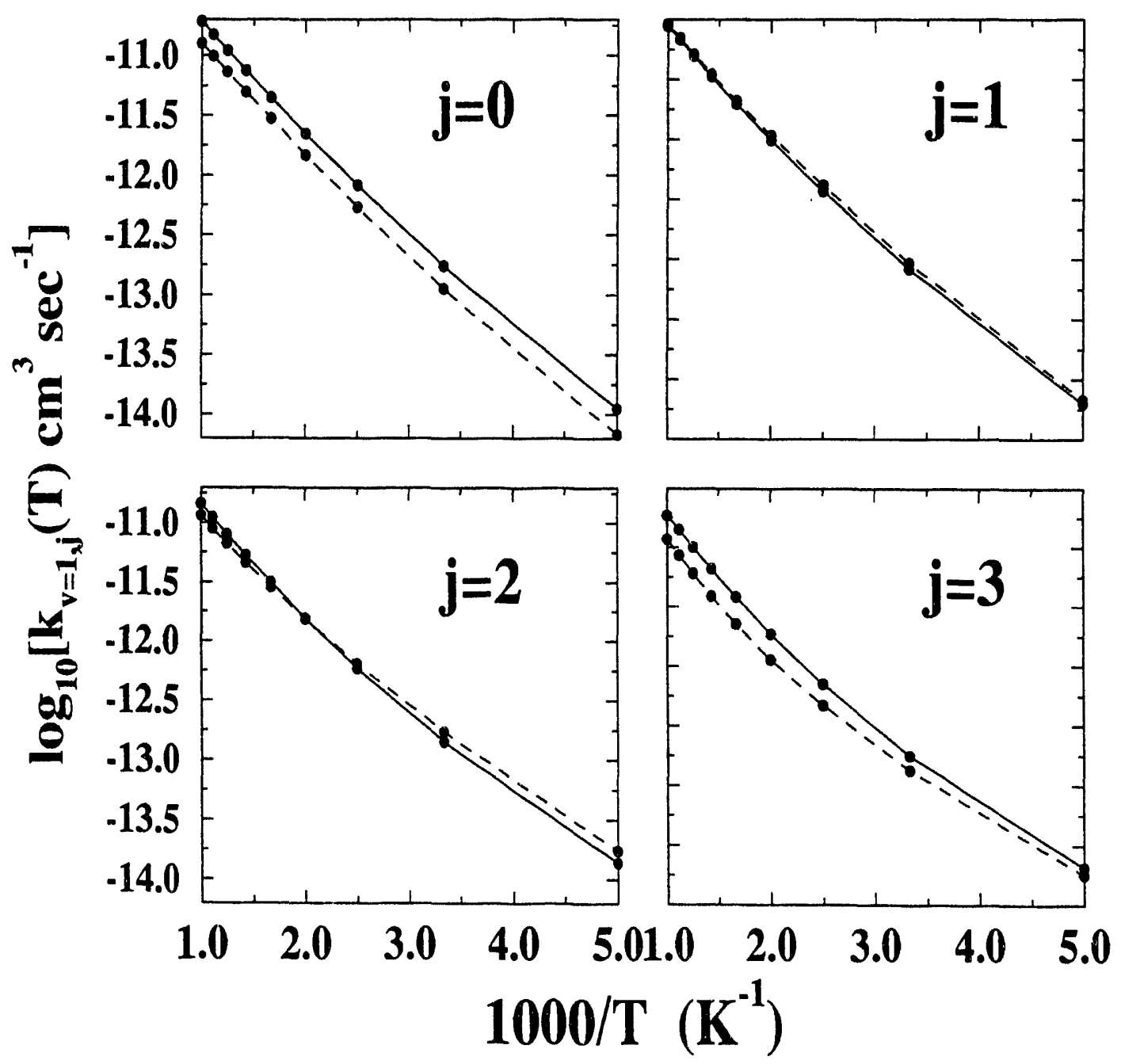

Figure 5.8: Exact and approximate (JSA) rate constants for the $\mathrm{D}+\mathrm{H}_{2}(v=1, j)$ reaction. The JSA predicts the correct Arrhenius activation energy (i.e. slope) in all cases, and is qualitatively correct in predicting the Arrhenius prefactor (i.e. yintercept). Agreement is excellent for $j=1$ and 2. However, the JSA systematically underestimates the rate constant by roughly $40 \%$ for $j=0$ and 3 . 


\begin{tabular}{||llll||}
\hline$\underline{j}$ & $\underline{\text { EXA }}$ & $\underline{\text { JSA }}$ & $\underline{p_{v=1, j}}$ \\
0 & 2.10 & 1.37 & $11.9 \%$ \\
1 & 1.98 & 2.23 & $63.6 \%$ \\
2 & 1.70 & 2.03 & $12.5 \%$ \\
3 & 1.31 & 0.976 & $11.2 \%$ \\
\hline
\end{tabular}

Table 5.2: Exact (EXA) and approximate (JSA) theoretical rate constants for $\mathrm{D}+\mathrm{H}_{2}(v=1, j)$ at $T=310 \mathrm{~K}\left(10^{-13} \mathrm{~cm}^{3}\right.$ molecule $\left.{ }^{-1} \mathrm{sec}^{-1}\right)$. Both EXA and JSA employ the ABC-DVR-Newton method. However, the JSA uses only $J=0$ reaction probabilities, and approximates the partial wave expansion. The final column represents the rotational mole fractions of $\mathrm{H}_{2}(v=1)$ at $T=310 \mathrm{~K}$. We note that although the JSA gives noticeable error, it is most accurate for the most populated state.

description of the experiment requires a thermal average over $j$-states. At $T=310 \mathrm{~K}$, $j=(0,1,2,3)$ accounts for $99.2 \%$ of the total population. We now present the results of an ABC-DVR calculation of these rate constants.

Table 5.2 shows $k_{v=1, j}(T=310 \mathrm{~K})$ with dimensions $10^{-13} \mathrm{~cm}^{3}$ molecule ${ }^{-1}$ $\sec ^{-1}$, for $j=(0,1,2,3)$. Both exact partial wave expansion and JSA are shown for comparison, in addition to the respective mole fractions of the $j$-states at $T=$ $310 \mathrm{~K}$. We note that the JSA is very reliable at predicting the order of magnitude of the rate constants. However, there is noticeable error, ranging from -34.8 to $+19.4 \%$. Furthermore, the JSA is poor at predicting the $j$ dependence of the exact rate constants at this temperature, decreasing with increasing $j$. However, the scatter in error and the fact that the most populated $j$-state is most accurately treated by the JSA suggests that it might do well to predict the average rate constant.

Table 5.3 shows the comparison between the CARS experiment, the present exact theory, and the JSA for the rotationally averaged rate constant at $T=310 \mathrm{~K}$, using the same units as in Table 5.2. As hoped (and expected!), the exact theory agrees quantitatively with the experimental result. Thus, we can truly regard the determination of the $\mathrm{D}+\mathrm{H}_{2}(v=1)$ rate constant as a solved problem in gas phase reaction dynamics. What is more intriguing, perhaps, is that the JSA predicts the 


\begin{tabular}{|c|c|}
\hline Method & $k_{v=1}(T=310 K)$ \\
\hline Experiment & $1.9 \pm 0.2$ \\
\hline EXA theory & 1.87 \\
\hline JSA theory & 1.95 \\
\hline
\end{tabular}

Table 5.3: Comparison between experiment, exact theory (EXA), and approximate theory (JSA) of rate constants for $\mathrm{D}+\mathrm{H}_{2}(v=1)$ at $T=310 \mathrm{~K}\left(10^{-13} \mathrm{~cm}^{3}\right.$ molecule $\mathrm{c}^{-1}$ $\left.\mathrm{sec}^{-1}\right)$. The theoretical values are obtained from Table 5.2 by averaging over the populated $j$-states. The experimental value is from Dreier and Wolfrum [Ref. 4]. Both the EXA and JSA rate constants agree quantitatively with experiment.

rate constant quantitatively as well. Clearly, from the analysis of Table 5.2, there is fortuitous cancellation of error in the average JSA rate constant. It is reasonable to question whether this cancellation obtains at all temperatures, or only in this temperature range.

To answer this question, we have computed the rotationally averaged rate constant as a function of temperature, comparing exact theory to the JSA result. The common logarithm of the resulting rate constants is plotted in thick lines against inverse temperature in Fig. 5.9. In addition, the exact and JSA $(v=1, j=0)$-selected rate constants are plotted in thin lines to demonstrate the systematic error. We see in Fig. 5.9 that the average JSA rate constant quantitatively predicts the exact one up to ca. $T=700 \mathrm{~K}$. At higher temperatures, the assumptions inherent in the JSA naturally tend to break down, as is manifest in Fig. 5.9. Thus, we have shown that for this system, the JSA gives the correct order of magnitude for the more detailed $[v=1, j=(0,1,2,3)]$-selected rate constants, and is semi-quantitative for the less detailed $(v=1,<j>)$-selected rate constant.

\subsection{Concluding remarks}

We have described what may be the most efficient full dimensional representation of a quantum reactive scattering system. This involves different representations 


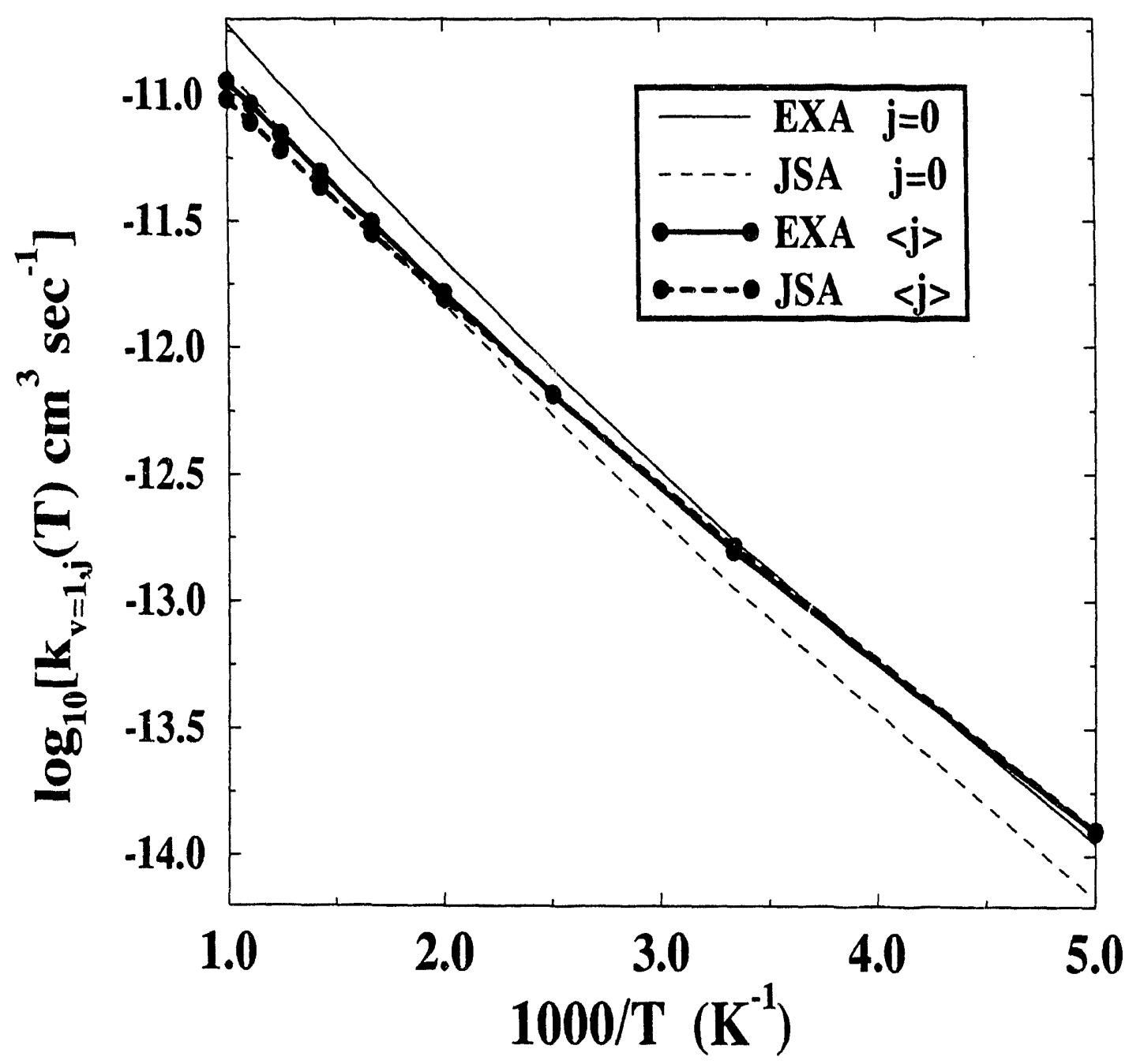

Figure 5.9: Comparison between exact theory (solid) and the $J$-shifting approximation (JSA-dash) of $\mathrm{D}+\mathrm{H}_{2}(v=1, j)$ rate constants for $\langle j\rangle$ (thick) and $j=0$ (thin) as a function of temperature. With respect to the $j=0$ rate constants, the JSA consistently underestimates the exact rate constant by ca. $35 \%$. However, with respect to the $\langle j\rangle$ rate constants, the JSA is nearly exact at the lower temperatures $T \leq 700 \mathrm{~K}$, and is semi-quantitative throughout the entire temperature range. Noticeable error occurs at the highest temperatures as the assumptions inherent in the JSA break down. 
for the interaction region and the asymptotic region. In the interaction region, we use a body-fixed reference frame for the overall rotation and a grid for the internal motions. In the asymptotic region, we use a space-fixed reference frame for the overall rotation and an absorbing potential to model outgoing wave boundary conditions for the internal motions. This approach is used to minimize vibration rotation coupling, and to allow the use of very efficient sparse matrix techniques to solve the scattering equations.

The ABC-DVR-Newton algorithm has been applied to the non-trivial probLun of determining accurate reaction cross sections for $\mathrm{D}+\mathrm{H}_{2}(v=1, j)$ over a wide energy range. These cross sections were found to have very smooth energy dependence, and to systematically decrease with increasing $j$. In favorable circumstantces, the present method gives converged reaction cross sections in 10 minutes on an IBM $\mathrm{RS} / 6000$ Model 550. In the most challenging cases, the calculations required no more than 60 minutes per energy (for $j=0$ ). In all cases, the core memory required was less than 4.5 Mbyte. We believe that the ABC-DVR-Newton method has all the necessary ingredients to move exact quantum reactive scattering calculations past the three atom problem.

The rate constants $k_{v=1, j}(T)$ were computed and thermally averaged over $j=(0,1,2,3)$ at $T=310 K$ to model the experiment by Dreier and Wolfrum [4]. Our result is $k_{v=1}(T=310 \mathrm{~K})=1.87 \times 10^{-13} \mathrm{~cm}^{3}$ molecule ${ }^{-1} \mathrm{sec}^{-1}$, in quantitative agreement with their value $(1.9 \pm 0.2) \times 10^{-13} \mathrm{~cm}^{3}$ molecule ${ }^{-1} \mathrm{sec}^{-1}$. We thus consider the subject of the $\mathrm{D}+\mathrm{H}_{2}(v=1)$ rate constant to be solved, with experiment and theory in complete agreement.

The $J$-shifting approximation (JSA) was tested against the exact $k_{v=1, j}(T)$ and $k_{v=1}(T)$ rate constants for $T=200-1000 K$. The $[v=1, j=(0,1,2,3)]$-selected JSA rate constants were qualitatively correct, but were in error by as much as $41 \%$. The error systematically cancelled for the $(v=1,<j>)$-selected rate constant, giving ;. semi-quantitative description of this averaged quantity for $T \leq 700 \mathrm{~K}$.

Although the most detailed attributes of the $\mathrm{D}+\mathrm{H}_{2}$ reaction are still under discussion, e.g. the geometric phase [56], we can confidently say that the average behavior of this system is well understood. 


\section{References}

[1] M. Keuba, U. Wellhausen, and J. Wolfrum, Ber. Bunsenges. Phys. Chem. 83, $940(1979)$.

[2] G. F. Glass and B. K. Chaturvedi, J. Chem. Phys. 77, 3478 (1982).

[3] V. B. Rozenshtegn, Yu. M. Gershenzon. A. V. Ivanov, and S. I. Kucheryavii, Chem. Phys. Lett. 105, 423 (1984).

[4] T. Dreier and J. Wolfrum, Int. J. Chem. Kinet. 18, 919 (1986).

[5] J. C. Sun, B. H. Choi, R. T. Poe, and K. T. Tang, Phys. Rev. Lett. 44, 1211 (1980).

[6] H. R. Mayne and J. P. Toennies, J. Chem. Phys. 75, 1794 (1981).

[7] R. B. Walker and E. F. Hayes, J. Phys. Chem. 87, 1255 (1983).

[8] J. M. Bowman, K. T. Lee, and R. B. Walker, J. Chem. Phys. 79, 3742 (1983).

[9] N. Abu Salbi, D. J. Kouri, Y. Shima, and M. Baer, J. Chem. Phys. 82, 2650 (1985).

[10] E. Pollack, N. Abu Salbi, and D. J. Kouri, Chem. Phys. Lett. 113, 585 (1985).

[11] E. Pollack, J. Chem. Phys. 82, 106 (1985).

[12] B. C. Garrett and D. G. Truhlar, J. Phys. Chem. 89, 2204 (1985).

[13] P. Siegbahn and B. Liu, J. Chem. Phys. 68, (1978) 2457.

[14] D. G. Truhlar and C. J. Horowitz, J. Chem. Phys. 68, (1978) 2466.

[15] D. G. Truhlar and C. J. Horowitz, J. Chem. Phys. 71, (1979) 1514(E).

[16] J. Z. H. Zhang and W. H. Miller, J. Chem. Phys. 91, 1528 (1989). 
[17] A brief summary of these calculations appeared previously in the following article:

S. M. Auerbach and W. H. Miller, J. Chem. Phys. 100, 0000 (1994).

[18] J. M. Bowman, J. Phys. Chem. 95, 4960 (1991).

[19] J. V. Micheal, J. R. Fisher, J. M. Bowman, and Q. Y. Sun, Science 249, 269 (1990).

[20] The JSA is essentially a molecular version of the modified wave number approximation of atomic physics. For this perspective, see:

K. Takayanagi, Prog. Theor. Phys. 8, 497 (1952);

E. E. Nikitin, Elementary Theory of Atomic and Molecular Processes in Gases (transl. by M. J. Kearsley), (Oxford University Press, New York, 1974), pp. $58-60$.

[21] D. C. Chatfield, R. S. Friedman, D. G. Truhlar, B. C. Garrett, and D. W. Schwenke, J. Am. Chem. Soc. 113, 486 (1991).

[22] S. Takada, A. Ohsaki, and H. Nakamura, J. Chem. Phys. 96, 339 (1992).

[23] A. Sinha, M. C. Hsiao and F. F. Crim, J. Chem. Phys. 94, 4928 (1991).

[24] M. J. Bronikowski, W. R. Simpson, B. Girard and R. N. Zare, J. Chem. Phys. 95, 8647 (1991).

[25] R. D. Levine and R. B. Bernstein, Molecular Reaction Dynamics and Chemical Reactivity (Oxford University Press, New York, 1987).

[26] T. Seideman and W. H. Miller, J. Chem. Phys. 96, 4412 (1992); 972499 (1992).

[27] W. H. Miller, Acc. Chem. Res. 26, 174 (1993).

[28] W. H. Thompson. and W. H. Miller, Chem. Phys. Lett. 206, 123 (1993).

[29] D. Neuhauser and M. Baer, J. Chem. Phys. 91, 4651 (1989). 
[30] A. Goldberg and B. W. Shore, J. Phys. B 11, 3339 (1978).

[31] C. Leforestier and R. E. Wyatt, J. Chem. Phys. 78, 2334 (1983).

[32] R. Kosloff and D. Kosloff, J. Comput. Phys. 63, 363 (1986).

[33] S. E. Choi and J. C. Light, J. Chem. Phys. 92, 2129 (1990).

[34] R. T. Pack, J. Chem. Phys. 60, 633 (1974).

[35] J. Z. H. Zhang, J. Chem. Phys. 94, 6047 (1991).

[36] M. E. Rose, Elementary Theory of Angular Momentum (John Wiley \& Sons, Inc., New York, 1967).

[37] D. O. Harris, G. G. Engerholm and W. D. Gwinn, J. Chem. Phys. 43, (1965) 1515.

[38] A. S. Dickinson and P. R. Certain, J. Chem. Phys. 49, (1968) 4209.

[39] J. C. Light, I. P. Hamilton and J. V. Lill, J. Chem. Phys. 82, (1985) 1400.

[40] G. C. Groenenboom, Report, Netherlands Organization for Scientific Research (NWO), 1992.

[41] G. C. Groenenboom and D. T. Colbert, J. Chem. Phys. 99, 0000 (1993).

[42] D. T. Colbert and W. H. Miller, J. Chem. Phys. 96, (1992) 1982.

[43] C. Leforestier, J. Chem. Phys. 94, 6388 (1991).

[44] G. C. Corey and D. Lemoine, J. Chem. Phys. 97, 4115 (1992).

[45] M. Abramowitz and I. A. Stegun, Handbook of Mathematical Functions with Formulas, Graphs and Mathematical Tables (Wiley, New York, 1972).

[46] G. C. Schatz and A. Kuppermann, J. Chem. Phys. 65, 4642 (1976).

[47] Z. Bačić and J. C. Light, J. Chem. Phys. 85, (1986) 4594. 
[48] W. H. Miller, J. Chem. Phys. 49, 2373 (1968).

[49] D. Neuhauser and M. Baer, J. Chem. Phys. 90, (1989) 4351.

[50] M. S. Child, Mol. Phys. 72, (1991) 89.

[51] Á. Vibók and G. G. Balint-Kurti, J. Phys. Chem. 96, (1992) 8712.

[52] Á. Vibók and G. G. Balint-Kurti, J. Chem. Phys. 96, (1992) 7615.

[53] S. M. Auerbach and C. Leforestier, Comput. Phys. Commun. 00, 0000 (1993).

[54] Q. Sun, J. M. Bowman, G. C. Schatz, J. R. Sharp, and J. L. Connor, J. Chem. Phys. 92, 1677 (1990).

[55] M. C. Colton and G. C. Schatz, Int. J. Chem. Kinet. 18, 961 (1986).

[56] A. Kuppermann and Y.-S. M. Wu, Chem. Phys. Lett. 205, 577 (1993). 


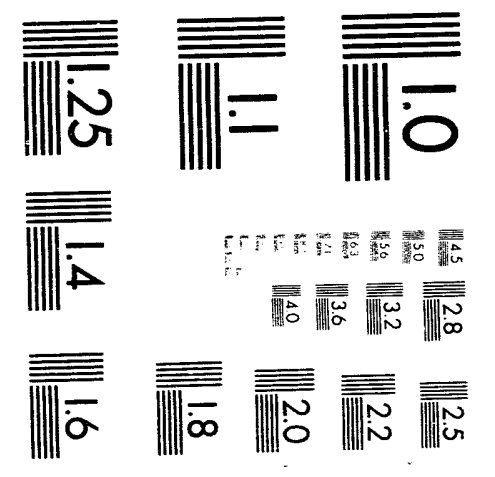





\section{Chapter 6}

\section{General Conclusions}

We conclude by summarizing the results presented in this dissertation, discussing their impact on the field of reaction dynamics, and suggesting future directions of research in theoretical chemistry.

\subsection{The $\mathbf{H} / \mathbf{D}+\mathbf{H}_{2}$ System}

We have analyzed the sensitivity of quantum reactive scattering calculations for $\mathrm{H}+\mathrm{H}_{2}$ to small changes in the molecular potential, and find no qualitative changes and very small quantitative changes in the resulting cross sections. The fact that these calculations (at least for $\mathrm{H}+\mathrm{H}_{2}$ ) do not show anomalous sensitivity helps to put $a b$ initio reaction dynamics on firm ground. It is true that the difference between the potentials considered in Chapter 2 is, in some sense, trivial. Nevertheless, the fact that the difference in the resulting dynamics is also trivial is an important result.

In addition, we have studied the initial state selected $\mathrm{D}+\mathrm{H}_{2}(v=1, j) \rightarrow$ $\mathrm{DH}+\mathrm{H}$ reaction. For the first time, the initial vibrationally excited rate constant $k_{v=1}(T=310 \mathrm{~K})$ agrees quantitatively with experiment. Based on these $\mathrm{D}+\mathrm{H}_{2}$ calculations, the $\mathrm{H}+\mathrm{H}_{2}$ calculations in Chapter 2, and the recent $\mathrm{D}+\mathrm{H}_{2}$ differential cross section calculations of Kuppermann and $\mathrm{Wu}$ [1] in their study of the gemetric phase effect, we conclude that many of the quantitative aspects of the $\mathrm{H}+\mathrm{H}_{2}$ reaction (and isotopic analogues) on its ground electronic state are well understood. 
A less well known system is the $\mathrm{F}+\mathrm{H}_{2} \rightarrow \mathrm{HF}+\mathrm{H}$ reaction. The potential for this system is characterized by an early barrier and a large exoergicity, and the dynamics involve complex formation. The early barrier provides an interesting study of the Polanyi rules, which predict that reaction is more favorable with translational energy on the $\mathrm{F}+\mathrm{H}_{2}$ side, or with vibrational energy of the $\mathrm{HF}+\mathrm{H}$ side. In addition, exoergic reactions are important for the development of new sources of fuel. Furthermore, reactions which form collision complexes facilitate the study of transition state structure (or in the language of particle physics, allow the study of new particles). Thus, the experimental and theoretical characterization of this reaction is an important step in reaction dynanics.

Experimental product state distributions $[2-8]$ observe $[\mathrm{HF}(v=2)]$ in excess of $[\mathrm{HF}(v=3)]$, whereas the most accurate theoretical descriptions [9-11] predict the opposite trend in the vibrational branching ratio. The discrepancy is attributed to error in the $\mathrm{F}+\mathrm{H}_{2}$ potential, which is known less well than the $\mathrm{H}+\mathrm{H}_{2}$ potential. In order to guide quantum chemists as they improve the $\mathrm{F}+\mathrm{H}_{2}$ potential, it may be useful to perform a functional sensitivity analysis for this system. This will pinpoint certain molecular configurations as being most important for determining the vibrational branching ratio. One hopes that the exisiting $\mathrm{F}+\mathrm{H}_{2}$ potential is not so inaccurate that it will point to the wrong configurations.

\subsection{Integral Equations}

We have developed two main integral equation approaches for quantum reactive scattering calculations. The physically important scattering solution to the Schrödinger equation has outgoing wave boundary conditions, which are automatically enforced by the Green's function-integral equation technique. The two methods we have discussed differ in how they apply the boundary conditions. The first method, the $S$-matrix version of the Kohn variational principle (KVP), constructs the outgoing wave Green's function by explicitly including in the Kohn basis asymptotically outgoing functions in all energetically accessible channels for all chemical arrangements. The second method, the absorbing boundary condition (ABC) formulation, 
constructs the outgoing wave Green's function effectively by absorbing all flux which leaves the interaction region. This absorption is effected by adding an empirical, negative imaginary absorbing potential to the physical Hamiltonian. As such, the KVP enforces the boundary conditions in the basis set, whereas the ABC formulation enforces the boundary conditions in the Hamiltonian operator. Both methods give a non-Hermitian Hamiltonian matrix, which is closely related to the complex coordinate rotation technique.

We have found the ABC formulation to be superior to the KVP for two reasons. First, the $\mathrm{ABC}$ formulation provides a single theoretical framework for directly computing the state-to-state reaction amplitude $S_{f i}(E)$, the initial state selected reaction probability $P_{i}(E)=\sum_{f}\left|S_{f i}(E)\right|^{2}$, and the cumulative reaction probability $N(E)$ $=\sum_{i} \sum_{f}\left|S_{f i}(E)\right|^{2}$. That is, by constructing the Green's function effectively, different levels of state resolution can be obtained, with concomitant amounts of computational effort. The second reason pertains to the structure of the Hamiltonian matrix. Since the $A B C$ formulation enforces boundary conditions in the Hamiltonian, no delocalized waves need to be included in the basis set. As such, a grid representation of the $\mathrm{ABC}$ Hamiltonian is sparse, which facilitates iterative linear system solving. On the other hand, because of the outgoing waves in the Kohn basis, a grid representation of the KVP Hamiltonian is essentially full, which severely limits the scope of its applicability. [We note that a sparse block of the KVP Hamiltonian can be projected out, but this requires that all initial conditions be considered, which also limits the scope of its applicability.] Thus, we advocate the use of $A B C$ in quantum reactive scattering theory.

The practicality of computing the ABC Green's function in a polynomial of the Hamiltonian is discussed. We find no feasible expansion which has a fixed and small memory requirement, and is guaranteed to converge. We have found, however, that exploiting the Fourier integral (i.e. time dependent) representation of the $A B C$ Green's function leads to a stable and efficient algorithm. We attribute this to the fact that by passing to the time domain, we can fully exploit the finite time nature of the collision event. And by construcing only finite time dynamics, we effectively remove the poles which make direct expansions unfeasible. Following Park and Light 
[12], one can think of the time variable as corresponding to the "number of iterations" in a Lanczos based method. Thus, the time domain provides a natural representation for developing iterative methods in quantum mechanics.

All the calculations reported in this dissertation involved relatively short time propagations $\left(T_{A B C} \leq 120 \mathrm{fs}\right.$ ). One may ask how efficient our scattering algorithm would be for treating reactions with long lived collision complexes (e.g. $T_{A B C} \geq$ $1 \mathrm{ps}$ ). In these cases, the conventional wisdom is to use a time independent method (e.g. the KVP or hyperspherical coordinate propagation). [In complex forming reactions, these "single energy" methods may give results quickly for each energy, but a very fine energy grid is required to trace out the energy dependence near the resonance.] However, the purpose of this dissertation is to develop methods for reactions which are sufficiently complicated (e.g. heavy masses or many atoms) that conventional methods, which obtain state-to-state amplitudes, are intractable. As such, we must treat the complex forming reaction with the $\mathrm{ABC}$ method, directly obtaining averaged reaction probabilities [13]. If the Hamiltonian can not be stored for $\mathbf{L U}$ decomposition, and GMRES does not converge because the condition number is large for resonant energies (cf. Chapter 4, Section 4.3.1), we are forced to use the time dependent Newton algorithm. Thus, we have returned to the time dependent picture, even for the treatment of long lived collision complexes.

Given this situation, is it computationally efficient to perform time dependent propagation on an energy dependent starting vector (ESV)? It does not seem efficient to run long propagations, each for a single energy only, when a single long wavepacket propagation provides dynamical information for a band of energies. An answer in favor of the ESV might claim that the ESV calculation provides the most direct route to dynamics at a single energy. However, this argument may not be compelling. Indeed, one can center an initial wavepacket in energy space around the energy of interest to obtain accurate dynamics for that energy. This wavepacket calculation may not require more effort than the corresponding ESV calculation. In addition, the wavepacket calculation contains dynamical information at other energies, albeit somewhat less accurately. Furthermore, as emphasized by Friesner et al. [14], the time dependent picture allows one to match the resolution in energy space 
obtained for the dynamical quantities with the amount of error in the underlying potential, or with the energy resolution of a corresponding experiment. The implication here is that a fully time dependent wavepacket formulation may be optimal.

In principle, time dependent formulations exist for computing the cumulative reaction probability $[16,17]$ and the thermal reaction rate constant [16]. However, an efficient implementation which gives results at many energies or temperatures, respectively, is difficult because of the mixed state nature of these dynamical quantities. On the other hand, efficient time dependent wavepacket $A B C$ formulations exist for computing the state-to-state reaction amplitude [10] and the initial state selected reaction probability [15]. Thus, if one is interested in state resolved reaction probabilities for systems which form long lived collision complexes, the time dependent wavepacket $A B C$ formulation is advocated.

The ESV calculations in Chapters 4 and 5 on the relatively direct $\mathrm{D}+\mathrm{H}_{2}$ reaction demonstrate remarkably rapid convergence. This appears to be an extremely attractive computational framework for solving the four atom reactive scattering problem in full dimensionality (i.e. six dimensions with $J=0$ ), allowing the $a b$ initio study of many reactions of interest to science $[18,19]$ and technology. Indeed, such studies are now underway [20].

\subsection{And Beyond ...}

\subsubsection{Quantum Reaction Rate Theory}

An important avenue of future research in quantum reactive scattering theory is the development of reduced dimensionality theories [21], which are able to estimate the reactivity of relatively complex systems by fixing or averaging over spectator modes. The difficult aspect of this work is in determining coordinate systems for which the spectator modes are indeed globally inert.

The use of quantum reactive scattering theory to predict rate constants for chemical reactions is presently relegated to the 3 or 4 atom world. It is crucial to extend reaction rate theory to larger systems. One possibility for this is to use classical 
mechanics. Although classical mechanics is surprisingly accurate for a wide variety of systems, it clearly lacks tunneling and zero point energy constraints which may be important for certain portions of the total system. As such, an interesting area of research involves the development and characterization of mixed quantum-classical methods [22, 23]. A time dependent dynamical formulation is useful here, since classical mechanics is most efficiently implemented as an initial value problem. The complicated aspect of this approach is isolating which modes are crucial to the reaction, and treating them with the appropriate mixture of quantum and classical mechanics.

\subsubsection{Gas Phase Reaction Dynamics}

Many exciting questions remain in gas phase reaction dynamics. The goal of bond and state selective chemistry is still not fully realized, although this type of control has been observed in the local mode $\mathrm{H}+\mathrm{H}_{2} \mathrm{O}$ system [18, 19]. An interesting new approach for chemical control involves preparing reactants in a coherent superposition of states [24], for which some theory already exists [25]. In applying these techniques to complex reactive systems, one hopes that the hard won coherence is not lost before the reaction proceeds.

As stated by Lee [26], using vibrational excitation to promote reactivity efficiently is difficult because intramolecular vibrational energy redistribution is so rapid. A fascinating question, then, pertains to the use of electronic excitation to promote reactivity. This may be important, for example, in studying the chemistry of nitrogen-oxygen compounds, which often have open shell configurations involving many interacting electronic states. From the theoretial perspective, performing a coupled surface quantum reactive scattering calculation remains challenging. Because the nonadiabatic coupling is singular at curve crossings, perturbation theory is not useful. The most convenient treatment involves diabatic electronic states, because diabatic couplings are more smooth. At present, though, surface hopping approaches are the method of choice [27]. 


\subsubsection{Physical Chemistry}

Physical chemistry is an enormous field, touching practically every form of matter. However, until now we have only discussed gas phase phenomena. A fundamental question, then, pertains to the applicability of gas phase principles to chemistry in liquid solution, on solid surfaces, in bulk solids, and in porous media. The basic question is: does reactivity drastically change from the effect of the medium, or is it only gently perturbed such that gas phase principles remain applicable? For example, canonical transition state theory (CTST) can be applied to a condensed phase system [28] by first isolating certain modes as constituting the "reactive system," with the remaining degrees of freedom providing a "bath." For fixed system configurations, one thermally averages over the bath, obtaining a free energy surface describing the adiabatic motion of the reacting system. Variational CTST rates are then computed by placing the dividing surface at the temperature dependent bottleneck in this free energy function. A very important issue is how to treat recrossing effects caused by the solvent. Calculating dynamical corrections is usually not feasible. An interesting new approach involves expanding the space of variational parameters used to optimize the dividing surface. In particular, Pollak [29] has shown that the Kramers-Grote-Hynes [30-32] treatment of solvent friction (i.e. recrossings) is really variational CTST. The challenging aspect of this approach is choosing which bath modes are most responsible for the recrossings. In any event, the development of a reliable, predictive model for these effects is of paramount importance.

One of the most important concepts in chemistry is catalysis. The development of effective catalysts requires knowledge of reaction paths and transition state structures. An important component in the future of physical chemistry, therefore, is the application of principles in reaction dynamics to the development of new materials with useful catalytic properties. I believe that the remarriage of physical chemistry and materials science will provide many years of excitement and discovery to come. 


\section{References}

[1] A. Kuppermann and Y.-S. M. Wu, Chem. Phys. Lett. 205, 577 (1993).

[2] J. H. Parker and G. C. Pimentel, J. Chem. Phys. 51, 91 (1969).

[3] J. C. Polanyi and D. C. Tardy, J. Chem. Phys. 51, 5717 (1969).

[4] N. Jonathan, C. M. Melliar-Smith, and D. H. Slater, Mol. Phys. 20, 92 (1971).

[5] J. C. Polanyi and K. B. Woodall, J. Chem. Phys. 57, 1574 (1972).

[6] R. D. Coombe and G. C. Pimentel, J. Chem. Phys. 59, 251 (1973).

[7] M. J. Berry, J. Chem. Phys. 59, 6229 (1973).

[8] D. M. Neumark, A. M. Wodtke, G. N. Robinson, C. C. Hayden, and Y. T. Lee, J. Chem. Phys. 82, 3045 (1985).

[9] J. M. Launay and M. L. Dourneuf, Chem. Phys. Lett. 169, 473 (1990).

[10] D. Neuhauser, R. S. Judson, R. L. Jaffe, M. Baer, and D. J. Kouri, Chem. Phys. Lett. 176, 5467 (1991).

[11] J. Z. H. Zhang, Chem. Phys. Lett. 181, 63 (1991).

[12] T. J. Park and J. C. Light, J. Chem. Phys. 85, 5870 (1986).

[13] An example is the complex forming $\mathrm{H}+\mathrm{O}_{2}$ reaction, for which the cumulative reaction probability was computed by the $\mathrm{ABC}$ method; see $\mathrm{C}$. Leforestier and W. H. Miller, J. Chem. Phys., submitted.

[14] R. A. Friesner, J. A. Bentley, M. Menou, and C. Leforestier, J. Chem. Phys. 99, 324 (1993).

[15] D. Neuhauser and M. Baer, J. Chem. Phys. 91, (1989) 4651.

[16] W. H. Miller, S. D. Schwartz, and J. W. Tromp, J. Chem. Phys. 79, 4889 (1983). 
[17] W. H. Miller, Acc. Chem. Res. 26, 174 (1993).

[18] A. Sinha, M. C. Hsiao and F. F. Crim, J. Chem. Phys. 94, 4928 (1991).

[19] M. J. Bronikowski, W. R. Simpson, B. Girard and R. N. Zare, J. Chem. Phys. 95, 8647 (1991).

[20] W. H. Thompson and W. H. Miller, J. Chem. Phys., in preparation.

[21] J. M. Bowran, J. Phys. Chem. 95, 4960 (1991).

[22] R. N. Barnett, U. Landman, S. Dhar, N. R. Kestner, J. Jortner, and A. Nitzan, J. Chem. Phys. 91, 7797 (1989).

[23] R. B. Gerber and R. Alimi, Isr. J. Chem. 31, 383 (1991).

[24] S. M. Park, S. P. Lu, and R. J. Gordon, J. Chem. Phys. 94, 8622 (1991).

[25] J. L. Krause, M. Shapiro, and P. Brumer, J. Chem. Phys. 92, 1126 (1990).

[26] Y. T. Lee, University Lecture, U. C. Berkeley, 1993.

[27] J. C. Tully and R. M. Preston, J. Chem. Phys. 55, 562 (1971).

[28] D. Chandler, J. Chem. Phys. 68, 2959 (1978).

[29] E. Pollak, J. Chem. Phys. 95, 533 (1991).

[30] H. A. Kramers, Physica 7, 284 (1940).

[31] P. Hänggi, P. Talkner, and Michal Borkovec, Rev. Mod. Phys. 62, 251 (1990).

[32] R. F. Grote and J. T. Hynes, J. Chem. Phys. 73, 2715 (1980). 

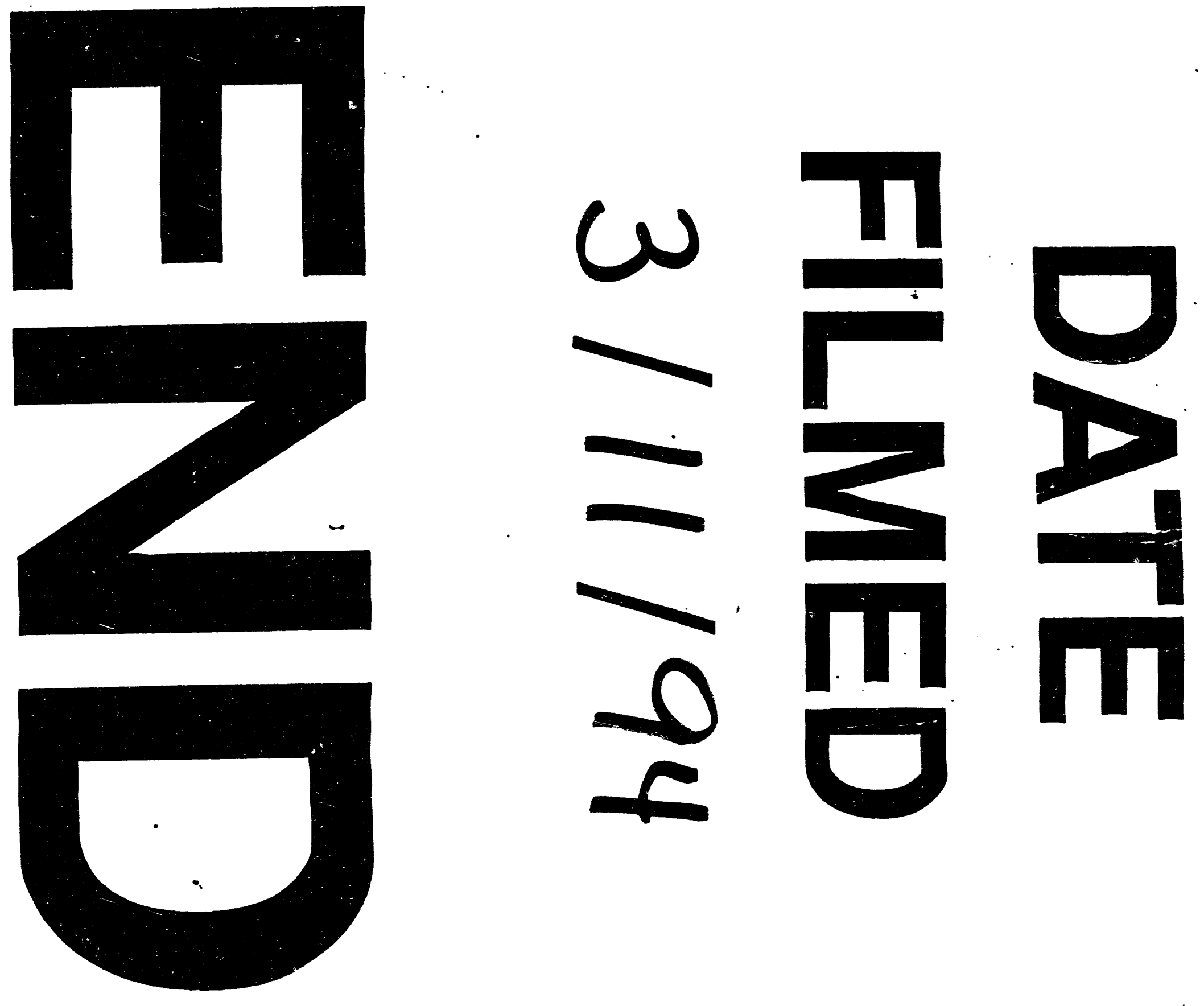